Aus der Klinik für Gastroenterologie und Gastrointestinale Onkologie der Medizinischen Fakultät der Universität Göttingen (Prof. Dr. med. V. Ellenrieder)

\title{
Frakturrisikoevaluation mithilfe verschiedener nationaler und internationaler Risikoscores bei Männern
}

\author{
INAUGURAL - DISSERTATION \\ zur Erlangung des Doktorgrades \\ der Medizinischen Fakultät der \\ Georg-August-Universität zu Göttingen \\ vorgelegt von \\ Judith Charlotte Witzel \\ aus \\ Rotenburg an der Fulda
}

Göttingen 2019 
Dekan:

I. Berichterstatter/in:

II. Berichterstatter/in:

III. Berichterstatter/in:

Datum der mündlichen Prüfung:
Prof. Dr. med. W. Brück

Prof. Dr. H. Siggelkow

PD Dr. Lukas Weiser

Prof. Dr. Margarete Schön

26.08.2020 
Hiermit erkläre ich, die Dissertation mit dem Titel „Frakturrisikoevaluation mithilfe verschiedener nationaler und internationaler Risikoscores bei Männern“ eigenständig angefertigt und keine anderen als die von mir angegebenen Quellen und Hilfsmittel verwendet zu haben.

Göttingen, den 


\section{Inhaltsverzeichnis}

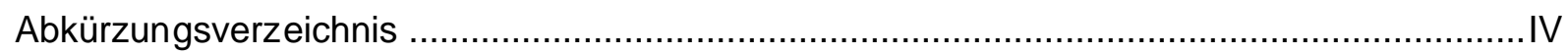

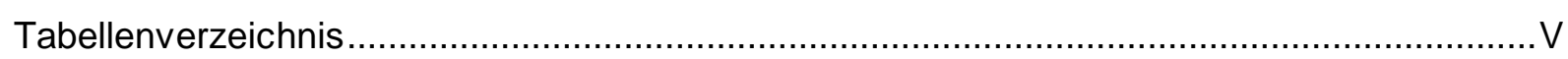

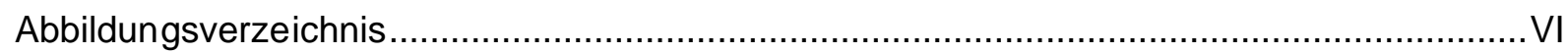

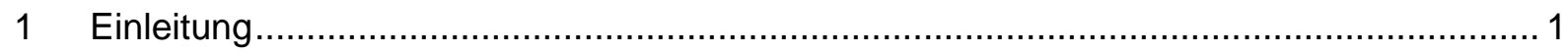

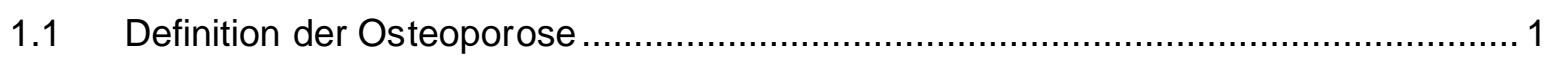

1.1.1 Epidemiologie und sozioökonomische Relevanz …................................... 1

1.1.2 Pathophysiologie und Klassifikation der Osteoporose ..................................... 3

1.1.3 Prävention und Therapie der Osteoporose nach Empfehlungen des DVO ........5

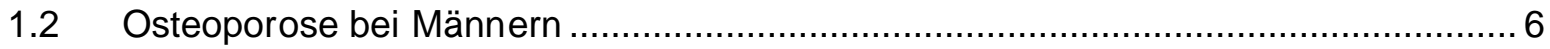

1.2.1 Primäre und sekundäre Osteoporose beim Mann .......................................... 7

1.2.2 Spezielle Therapie der Osteoporose beim Mann ......................................... 8

1.3 Nationale und internationale Scores zur Evaluation des osteoporoseassoziierten

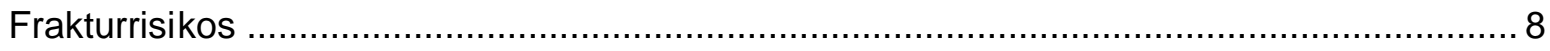

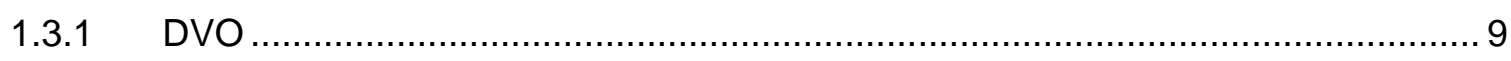

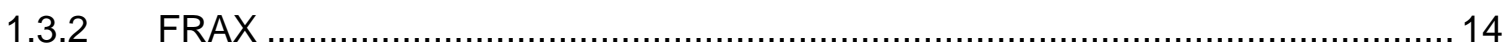

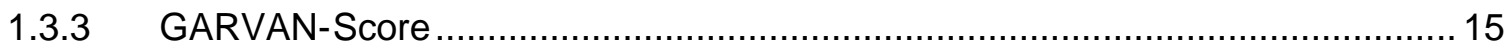

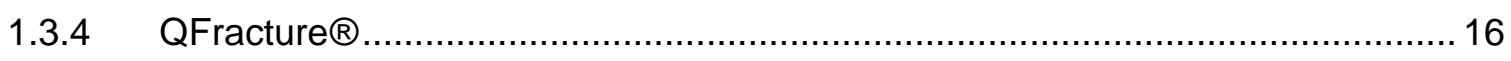

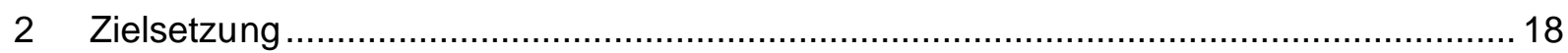

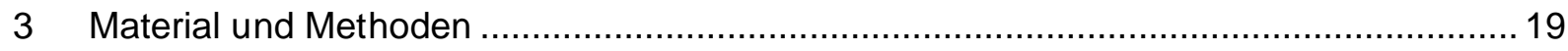

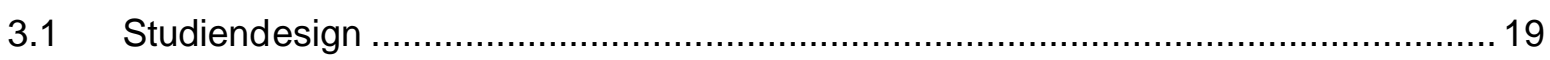

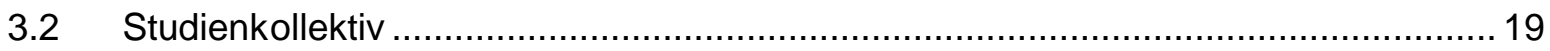

3.3 Genehmigung durch die Ethikkommission ..................................................... 19

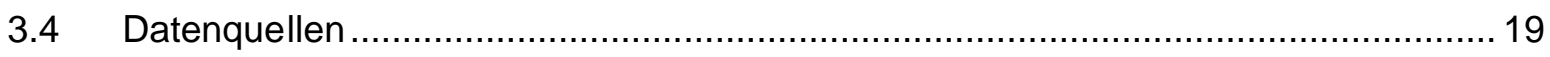

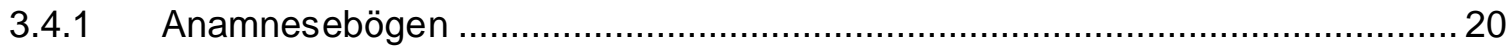

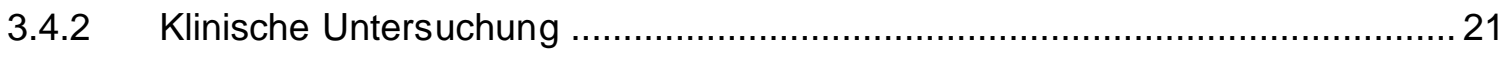

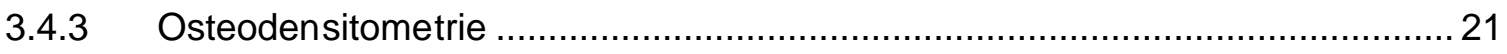

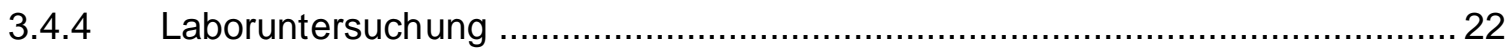

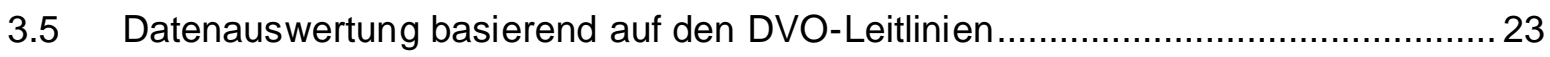




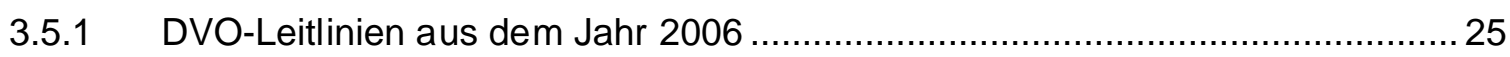

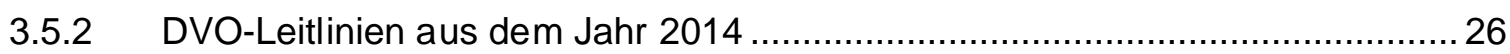

3.5.3 Datenauswertung basierend auf dem internetbasierten FRAX-Rechner ...........28

3.5.4 Datenauswertung basierend auf dem 1-Jahres-Frakturrisiko nach C. Glüer .... 30

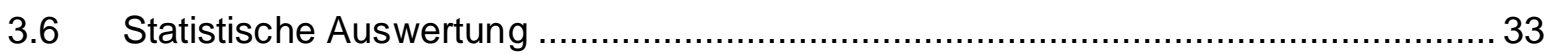

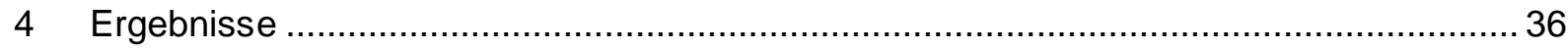

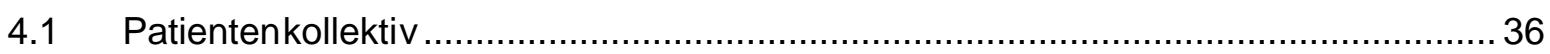

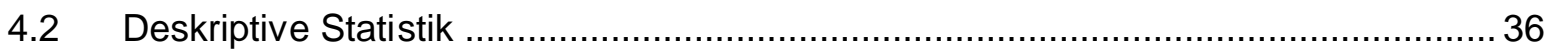

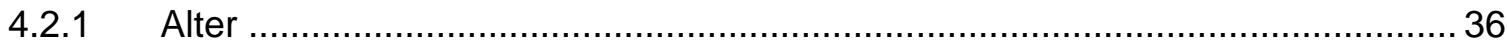

4.2.2 Körpergröße, Gewicht, Body Mass Index (BMI) .......................................... 37

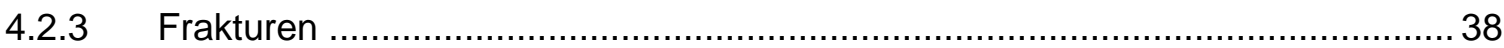

4.2.4 Femurfraktur bei einem der beiden Elternteile …....................................... 39

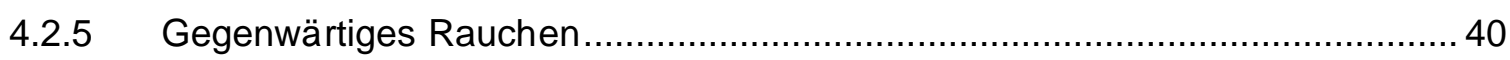

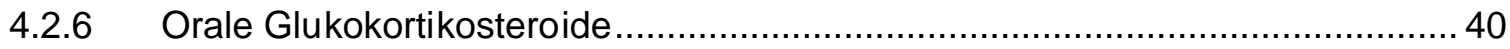

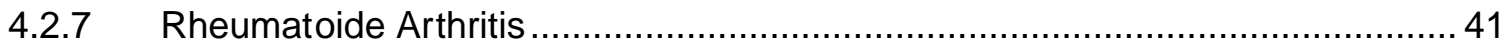

4.2.8 Sekundäre Osteoporoseursachen .......................................................... 41

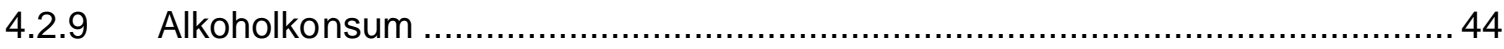

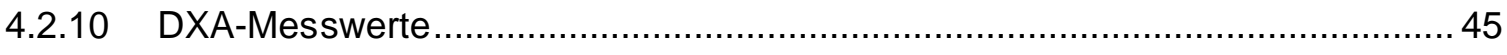

4.3 10-Jahres-Frakturrisiko nach den DVO-Leitlinien aus den Jahren 2006, 2009 und 2014 46

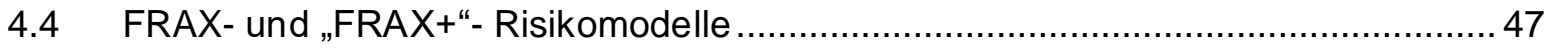

4.4.1 Therapieempfehlungen basierend auf den FRAX- Risikomodellen .................. 49

4.5 1-Jahres-Frakturrisiken nach C. Glüer ............................................................. 50

4.5.1 Therapieempfehlungen basierend auf dem Score nach C. Glüer ..................... 51

4.5.2 Hochrechnung des 1-Jahres-Frakturrisiko auf ein 10-Jahres-Frakturrisiko ......52

4.6 Vergleichende Betrachtung der 10-Jahres-Frakturrisiken des DVO-, FRAX-,

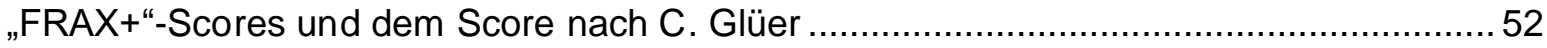

4.6.1 Vergleichende Betrachtung der Therapieindikationen zwischen dem DVO-Score aus den Jahren 2006-2014, des Scores nach C. Glüer und der FRAX-Scores 55

4.7 Vergleichende Betrachtung der Therapieempfehlungen basierend auf den WHOKriterien und dem DVO-Score, FRAX-Score, „FRAX+“-Score bzw. Score nach C. Glüer . 64 
4.8 Multiple lineare Regression 64

4.8.1 Lineares Regressionsmodell für das FRAX-10-Jahres-Frakturrisiko einer „major osteoporotic"Fraktur ohne und mit Einbeziehung der Knochendichtemessung 64

4.8.2 Lineares Regressionsmodell für das FRAX-10-Jahres-Frakturrisiko einer Hüftfraktur ohne und mit Einbeziehung der Knochendichtemessung. 66

4.8.3 Lineares Regressionsmodell für das „FRAX+“ 10-Jahres-Frakturrisiko einer MOF ohne und mit BMD 67

4.8.4 Lineares Regressionsmodell für das „FRAX+“-10-Jahresrisiko einer HF ohne und mit BMD 68

5 Diskussion 70

5.1 Studienkollektiv und Risikofaktorenanalyse 71

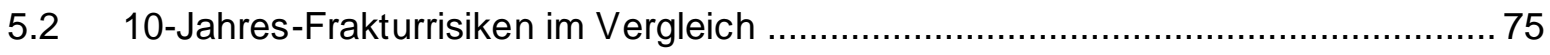

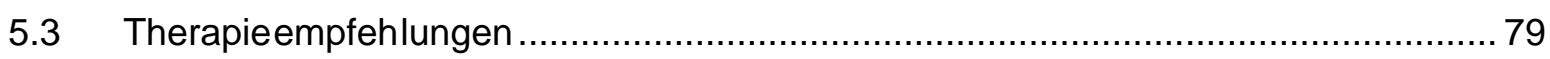

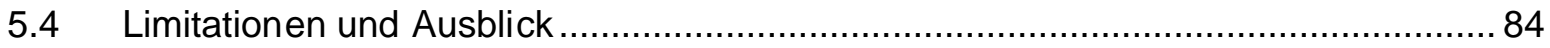

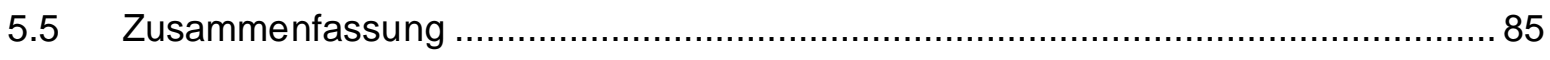

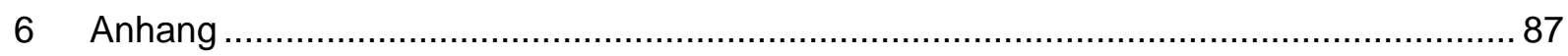

6.1 Modifizierung der Therapiegrenze nach den DVO-Leitlinien 2014 (DVO Leitlinien 2014) 87

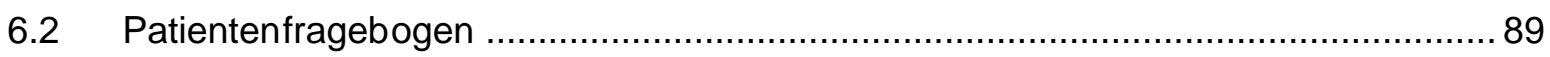

6.3 Osteoporose-Risikofragebogen für Patienten .......................................................... 93

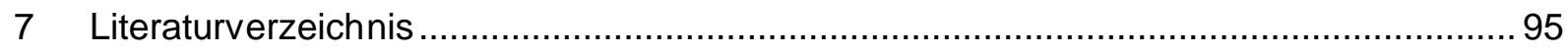




\section{Abkürzungsverzeichnis}

AWMF

Arbeitsgemeinschaft der Wissenschaftlichen Medizinischen Fachgesellschaften

BMD

bone mineral density

DVO

Dachverband Osteologie

DXA

dual energy $X$-ray absorptiometry

FRAX

Fracture Risk Assessment Tool

G-I-N

Guidelines International Network

HF

Hüftfraktur

IQR

Interquartilenabstände

MOF

major osteoporotic fracture

MVZ

medizinisches Versorgungszentrum

NICE

National Institute for Health and Care Excellence

NIH

National Institutes of Health

SD

standard deviation

SIGN

Scottish Intercollegiate Guidelines Network

WHO

World Health Organization 


\section{Tabellenverzeichnis}

Tabelle 1: Allgemeine Risikofaktoren für ein erhöhtes Risiko osteoporotischer Frakturen ..... 4

Tabelle 2: Mögliche Ursachen der sekundären Osteoporose ............................................ 5

Tabelle 3: Indikatoren für die Initiierung einer osteoporosespezifischen Diagnostik ............ 10

Tabelle 4: Indikation für eine spezifische medikamentöse Therapie .................................... 12

Tabelle 5: Indikationen für eine spezifische medikamentöse Therapie in Abhängigkeit von Geschlecht, Alter und DXA-Knochendichte .... .24

Tabelle 6: Risikofaktoren, die eine Anhebung der Therapiegrenze bedingen ......................25

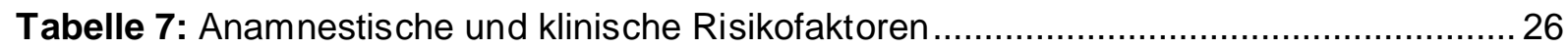

Tabelle 8: Neuerungen der Risikofaktoren, die die Therapiegrenze um $+0,5$ anheben ........ 27

Tabelle 9: Übersicht der Diagnoseschlüsse für eine sekundäre Osteoporose laut „FRAX+“-

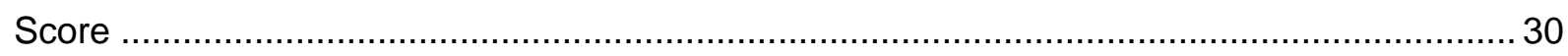

Tabelle 10: Risikofaktoren für die Kalkulation des 1-Jahres-Frakturrisiko nach C. Glüer ...... 31

Tabelle 11: Diagnoseverschlüsselung der sekundären Osteoporosen ................................ 42

Tabelle 12: Deskriptive Statistik FRAX- und „FRAX+“-10-Jahres-Fralturrisiken.................... 48

Tabelle 13: Korrelationskoeffizienten der FRAX- und „FRAX+“-Scores ................................. 48

Tabelle 14: Therapieempfehlungen ,ja“ basierend auf den FRAX-Modellen .........................50

Tabelle 15: 10-Jahres-Frakturrisiken des DVO-, FRAX- und „FRAX+“-Scores sowie des

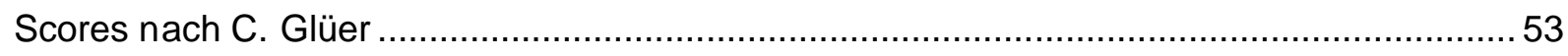

Tabelle 16: Mittelwerte der FRAX-Scores bzw. des Scores nach C. Glüer ..........................54

Tabelle 17: Korrelationskoeffizienten der Fraktur-Scores ................................................ 55

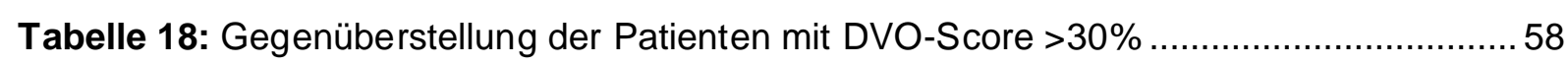

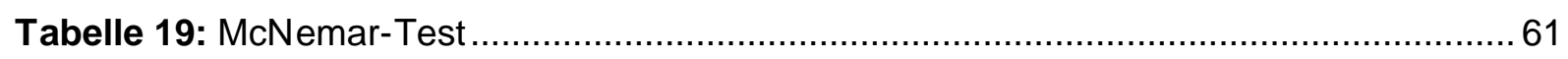

Tabelle 20: Übereinstimmung der Therapieentscheidungen in $\%$....................................... 63

Tabelle 21: Regressionsmodell FRAX-Score MOF ohne bzw. mit BMD ................................65

Tabelle 22: Regressionsmodell FRAX-Score Hüftfraktur ohne bzw. mit BMD ......................67

Tabelle 23: Regressionsmodell für den „FRAX+“-Score für eine MOF ohne bzw. mit BMD . 68

Tabelle 24: Regressionsmodell für den „FRAX+“-Score einer HF ohne bzw. mit BMD ........69

Tabelle 25: Gegenüberstellung der wichtigsten Gemeinsamkeiten und Unterschiede der

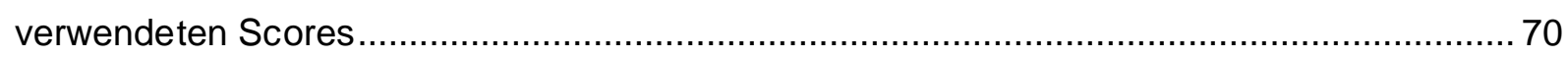

Tabelle 26: Vergleich der Basisdaten aus dem MVZ endokrinologikum Göttingen, der „MrOS“Studie und den Daten von Marques et al .................................................................... 72

Tabelle 27: Vergleich der durchschnittlichen FRAX-10-Jahres-Frakturrisken ....................... 76

Tabelle 28: Vergleich der Mediane und 25-\% bzw. 75-\% Interquartilenabstände (IQR) ....... 76

Tabelle 29: Therapiegrenzen FRAX-Score basierend auf den Daten des MVZ endokrinologikum 


\section{Abbildungsverzeichnis}

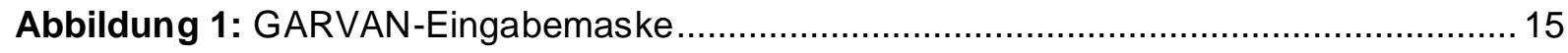

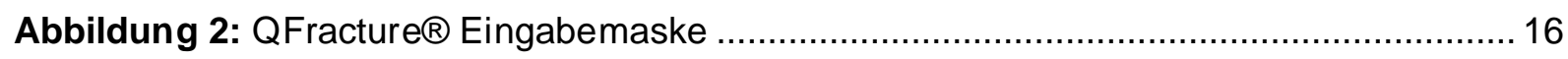

Abbildung 3: Lebensgewohnheiten im Kontext der osteoporosespezifischen Anamnese ... 21

Abbildung 4: Anamnestische und klinische Risikofaktoren .............................................. 24

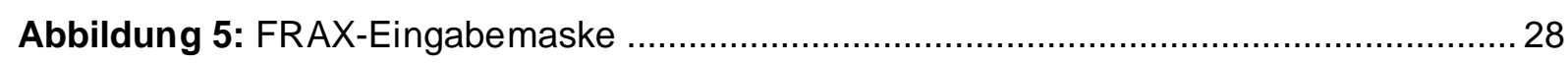

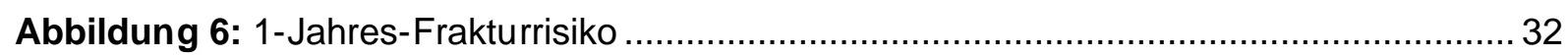

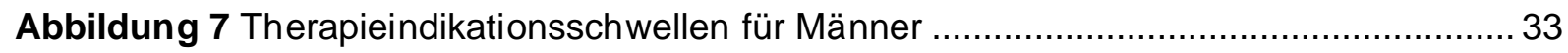

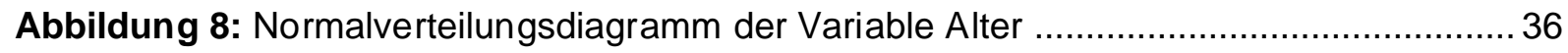

Abbildung 9: Boxplots: Gewicht (in kg) und Körpergröße (in cm) .................................... 37

Abbildung 10: Anzahl der Patienten pro Körpergewichtsklasse ........................................ 38

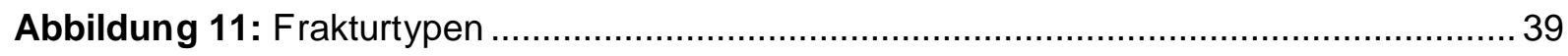

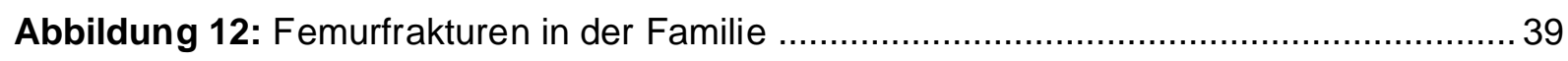

Abbildung 13: Gegenwärtiger Nikotinkonsum pro Tag .................................................. 40

Abbildung 14: Angaben zur Einnahme oraler Glukokortikoide....................................... 41

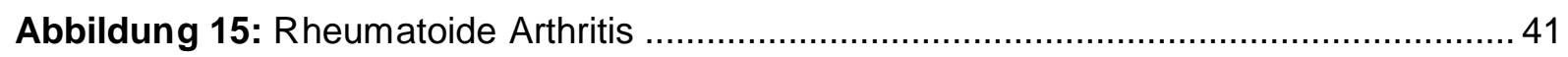

Abbildung 16: Häufigkeitsverteilung der sekundären Osteoporoseursachen ...................... 43

Abbildung 17: Alkoholeinheiten pro Tag pro Patient ...................................................... 44

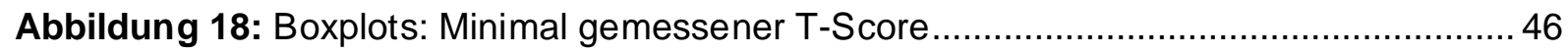

Abbildung 19: Anzahl der Patienten gruppiert nach prozentualen 10-Jahres Frakturrisiken 47 Abbildung 20:1-Jahres-Frakturrisiko nach dem Modell von C. Glüer ................................ 51

Abbildung 21: Gegenüberstellung der Therapieempfehlungen...........................................56

Abbildung 22: Patientenbezogene Therapieübereinstimmungen bzw. Unterschiede zwischen den einzelnen 10-Jahres-Frakturrisiko-Scores. 60 


\section{Einleitung}

\subsection{Definition der Osteoporose}

Versucht man die Diagnose "Osteoporose" auf ihre elementaren Charakteristika zu reduzieren, so lässt sie sich als eine systemische, skelettale Erkrankung mit einer verminderten Beanspruchungsfähigkeit der Knochen beschreiben. Die Beanspruchungsfähigkeit der Knochen wiederum leitet sich von der Knochendichte und Qualität der Knochenstruktur ab. Diese Faktoren werden durch pathologische Umwandlungsprozesse in der Mikroarchitektur des Knochens beeinflusst, welche wiederum in Verbindung mit einer erhöhten Knochenbrüchigkeit stehen (NIH 2001; Tu et al. 2018; Weaver et al. 2016). Eine erhöhte Knochenbrüchigkeit ist ein begünstigender Faktor für das Auftreten von Frakturen. Sobald eine pathologische Veränderung der Knochenbeanspruchungsfähigkeit in Kombination mit einem dadurch bedingten atraumatischen oder niedrigtraumatischen Knochenbruch vorliegt, spricht man von einer klinisch manifesten Osteoporose. Prädilektionsstellen für osteoporoseassoziierte Knochenbrüche sind der Femur, die Wirbelkörper, Oberschenkelknochen, Unterarm, proximaler Humerus, Hüfte, Tibia, Rippen und Schlüsselbein (Leitlinie Osteoporose 2017).

Um die Diagnose zu stellen, hat die World Health Organization (WHO) im Jahre 1994 auf einer Konsensuskonferenz die Knochendichte, gemessen mittels „Dual Energy X-Ray Absorptiometry“ (DXA), als Surrogatparameter zur Definition der Osteoporose angeführt. Demnach lässt sich eine Osteoporose diagnostizieren, sofern ein gemessener T-Score an der Lendenwirbelsäule, dem Gesamtfemur oder dem proximalen Femurhals $\leq-$ 2,5 Standardabweichung vom statistischen Mittelwert gesunder 20- bis 29-jährigen Frauen liegt (Kanis et al. 1994; WHO 1991, 2003). Mittels DXA Messung lässt sich der Kalksalzgehalt des Knochens bestimmen. Dieser ist jedoch nur ein Faktor aus einer Vielzahl an Risikofaktoren, die mit einem erhöhten Frakturrisiko einhergehen. Mittlerweile steht eine Vielzahl an Frakturrisikoscores zur Verfügung, deren Ziel es ist, anhand eines gewählten Sets an Risikofaktoren, welches nicht zwingend die Knochendichte einbezieht, das Frakturrisiko für osteoporoseassoziierte Frakturen abzuschätzen (Leitlinie Osteoporose 2014; Kanis et al. 2017).

\subsubsection{Epidemiologie und sozioökonomische Relevanz}

Laut WHO leiden weltweit ca. 6\% aller Männer und 21\% aller Frauen zwischen dem 50. und 84. Lebensjahr an einer Osteoporose. Die WHO zählt die Osteoporose zu einer der vier Hauptgefahren der menschlichen Gesundheit, neben Krebs, kardiovaskulären Erkrankungen und Schlaganfällen (Hernlund et al. 2013; Shariati-Sarabi et al. 2018). Jede zweite Frau und jeder fünfte Mann über 50 Jahre wird in den kommenden Lebensjahren eine Fraktur erleiden, die auf eine qualitativ minderwertige Knochenarchitektur zurückzuführen ist. Speziell für 
Femurfrakturen zeigt sich eine assoziierte Mortalität im ersten Jahr zwischen $8 \%$ und $36 \%$. Über 50\% der von dieser Frakturart betroffenen Patienten erlangen nicht mehr ihre vollständige physische Konstitution wieder (Lorentzon und Cummings 2015; Misiorowski 2017).

Sowohl die mit der Osteoporose assoziierten Frakturhäufigkeiten als auch die so entstehenden Kosten werden durch den demographischen Wandel ansteigen. Im europäischen Vergleich werden die meisten damit verbunden Frakturen (928 000) im Jahr 2025 in Deutschland erwartet. Darauf folgt, mit 682000 Frakturen, das Vereinigten Königreich. Insgesamt wird in Europa ein Anstieg von 3,5 Millionen osteoporoseassoziierten Frakturen im Jahr 2010 auf 4,5 Millionen im Jahr 2025 erwartet. Die aufkommenden Kosten werden für 2025 mit 46,8 Milliarden Euro in ganz Europa veranschlagt. Sie setzen sich zusammen aus den im ersten Jahr durch die Fraktur entstandenen Kosten, den Folgekosten und den Ausgaben für medikamentöse Interventionen und den damit in Verbindungen stehenden administrativen Vorgängen. Für das Jahr 2010 umfassten sie 37,4 Milliarden Euro. Den größten Anteil daran hatten mit 66\% die im ersten Jahr entstanden Kosten. Der geringste Anteil mit 6\% ergab sich in 2010 durch die Ausgaben für medikamentöse Therapieinterventionen (Hernlund et al. 2013).

Der BEST-Studie aus dem Jahre 2013 lassen sich osteoporosespezifische epidemiologische Angaben für über 50-jährige Bürger in Deutschland aus den Jahren 2006 bis 2009 entnehmen. Die Studie basiert auf einer retrospektiven Auswertung anonymisierter randomisierter Patientendaten der Techniker Krankenkasse. Unter Extrapolation der analysierten Daten, ergibt sich eine Prävalenz der Osteoporose für Deutschland von 6,3 Millionen, davon 5,2 Millionen Frauen und 1,1 Millionen Männer. Demzufolge ist jede vierte Frau und jeder siebzehnte Mann in Deutschland von der Krankheit betroffen. Die Inzidenzrate liegt laut der Studie bei 2,1\% (885 000 Neuerkrankungen) pro Jahr. Im Beobachtungszeitraum erlitten 52\% der betroffenen Patienten des Studienkollektives (172 473 Patienten) Frakturen. Bei der Großzahl der Pateinten traten multiple Frakturen auf (Hadji et al. 2013b).

Laut dem „Medizinischen Dienst der Krankenkassen“ beliefen sich die mit der Osteoporose in direkter Verbindung stehenden Kosten in Deutschland im Jahr 2003 auf 5,4 Milliarden Euro. Dieser Betrag entspricht 3,5\% aller entstandenen Gesundheitskosten der privaten- und öffentlichen Versicherungen im Jahr 2003. 56\% (3 Milliarden Euro) der Kosten entfielen dabei auf stationäre Behandlung der Patienten, 17\% (894 Millionen Euro) auf die Langzeitpflege und 15\% (791 Millionen Euro) auf die medikamentöse Behandlung. Den größten Posten der Medikamente nahmen dabei die Analgetika mit 62,9\% der Gesamtkosten ein, gefolgt von den osteoporosespezifischen Medikamenten mit 22,7\%. Im Schnitt verursachte ein Patient mit stattgehabter Fraktur im Jahr 2003 einen Kostenaufwand von 9962 Euro, ein Patient ohne Fraktur 281 Euro (Häussler et al. 2007; Häussler et al. 2006). 


\subsubsection{Pathophysiologie und Klassifikation der Osteoporose}

Die Knochenfestigkeit wird durch die Knochenmasse, sowie deren geometrische Zusammensetzung bestimmt. Die Kombination dieser Faktoren determiniert, ob es sich um eine kortikale oder spongiöse Knochenstruktur handelt. Kortikale Knochenstrukturen finden sich in etwa $80 \%$ des Knochenskeletts. Die Differenzierung zwischen spongiösem und kortikalen Knochenstrukturen ist im Kontext der pathophysiologischen Veränderungen relevant. So zeigt sich, dass metabolische Prozesse im Alter am kortikalen Knochen überwiegen. Dieser Prozess ist besonders ausgeprägt bei postmenopausalen Frauen. Am spongiösen Knochen zeigt sich bedingt durch die Altersprozesse eine Zunahme der Porosität (Brandi 2009; Chen et al. 2013).

Der Grundstein für eine intakte, normgerechte Knochenentwicklung, entsprechend der genetischen Variationsbreite, wird bereits in der frühen Kindheit und Jugend gelegt. Nur unter optimalen nutritiven Bedingungen, in Abwesenheit von schwerwiegenden Erkrankungen, ohne den Einfluss schädlicher Noxen oder Medikamente und regelmäßiger körperlicher Aktivität wird die individuell mögliche peak bone mass erreicht. Sie ist der Ausgangspunkt für die weitern Lebensjahre. Die individuelle Variationsbreite der peak bone mass wird durch genetische Faktoren vorgegeben. Zwillingsstudien ergeben, dass bis zu $80 \%$ der Variation der Knochendichte genetisch bedingt ist (Misiorowski 2017; Weaver et al. 2016).

Auch die Geschlechtsangehörigkeit und die Ethnie fließen als genetisch determinierende Faktoren mit ein. So haben kaukasische Frauen ein doppelt so hohes Erkrankungsrisiko wie Männer derselben Ethnie. Auch im weltweiten Vergleich haben hellhäutige Frauen das höchste Risiko, an einer Osteoporose zu erkranken, gefolgt von den Hispanos, Asiatinnen und Afroamerikanerinnen. Der Ethnienunterschied fällt bei Männern geringer aus als bei Frauen, trotz alledem zeigen sich auch hier höhere Prävalenzen für hellhäutige Männer als für Asiaten oder Afroamerikaner. Insgesamt betrachtet lassen sich die höchsten Knochendichten bei Männern afroamerikanischer Abstammung finden (Cauley 2011).

Mit zunehmendem Alter und somit erhöhter Exposition gegenüber Umweltfaktoren nimmt der genetische Einfluss auf die Knochendichte ab und der Einfluss äußerer Faktoren zu (Weaver et al. 2016). Abhängig vom Lebensstil und den Vorerkrankungen sowie damit verbundene Medikation lässt sich für jeden Patienten ein individuelles Risikoprofil erstellen. Wichtige allgemeine Faktoren, die mit einem erhöhten Risiko für osteoporotische Frakturen einhergehen, sind in Tabelle 1 gelistet. 

2014)

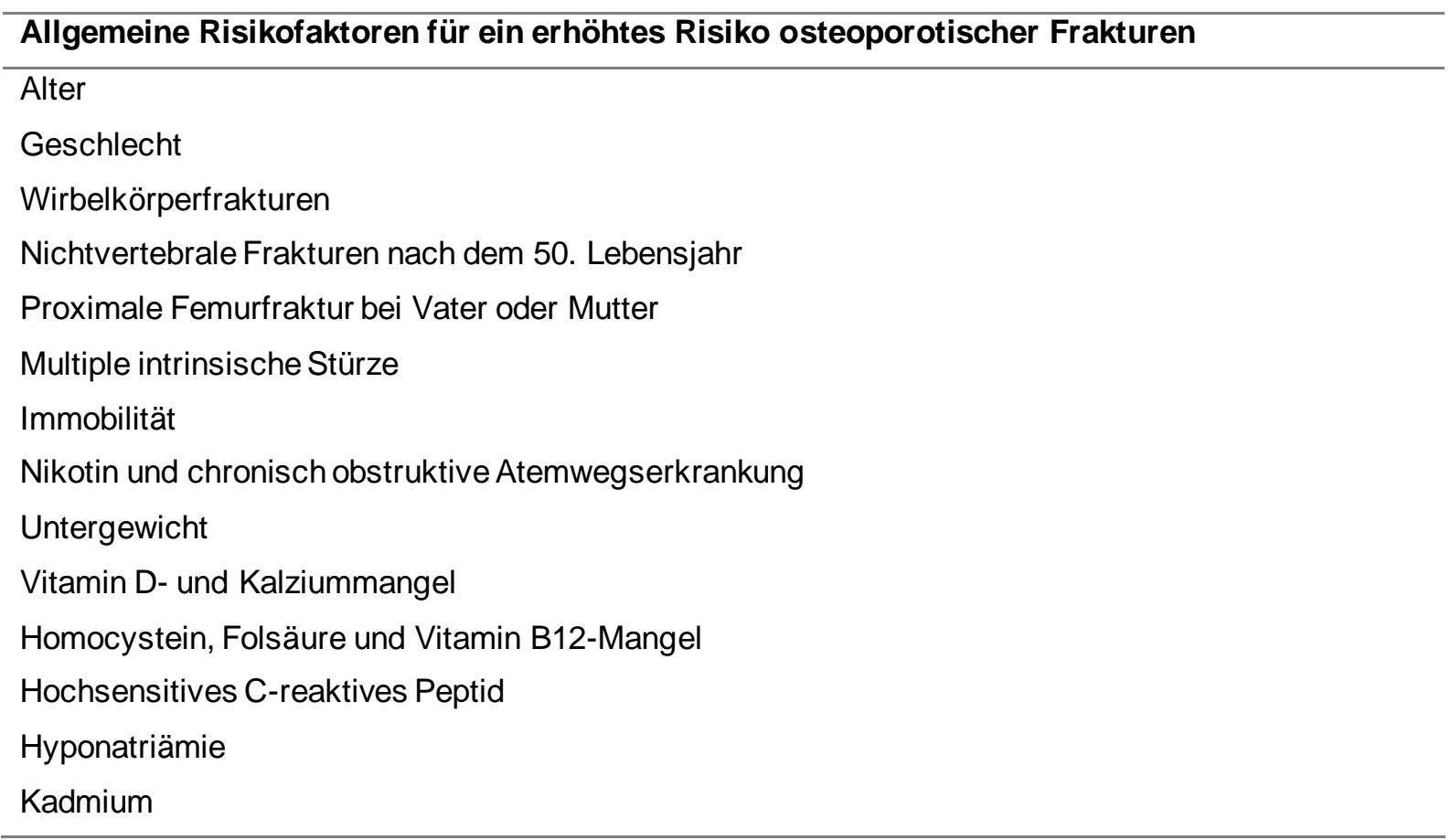

Basierend auf den pathophysiologischen Prozessen ist eine Klassifizierung in eine primäre und sekundäre Osteoporose möglich. Die primäre oder auch idiopathisch genannte Osteoporose umfasst vordergründig die postmenopausale und senile Form der Osteoporose. Sie geht einher mit natürlichen Alterungsprozessen und damit verbundenen hormonellen und strukturellen Prozessen. Begünstigend sind demzufolge eine früh eintretende Menopause, ein prämenopausales Östrogendefizit, aber auch Mangel- bzw. Fehlernährung (mit eingeschlossen sind Nikotinabusus und Alkoholkonsum), eine unzureichende Calciumzufuhr und Bewegungsmangel (Bartl und Bartl 2011; Tu et al. 2018).

Der sekundären Osteoporose liegt eine definitive Erkrankung oder Pathologie zugrunde, die in einer verminderten Knochendichte oder erhöhten Knochenbrüchigkeit resultiert. Je nachdem, ob nur ein Krankheitsbild oder mehrere zugrunde liegen, spricht man von einer monoätiologischen oder polyätiologischen sekundären Osteoporose. Zur Gruppe der begünstigenden Faktoten einer sekundären Osteoporose zählen ebenfalls Medikamente. Eine Auswahl möglicher Faktoren ist in der Tabelle 2 aufgeführt. 
Tabelle 2: Mögliche Ursachen der sekundären Osteoporose (Leitlinie Osteoporose 2014)

\begin{tabular}{ll}
\hline Osteoporosebegünstigende Erkrankungen & Osteoporosebegünstigende Medikamente \\
\hline $\begin{array}{l}\text { Cushing-Syndrom und subklinischer Hyper- } \\
\text { kortisolismus }\end{array}$ & Antiandrogene Therapie bei Männern \\
Primärer Hyperparathyreodismus & Antidepressiva \\
Wachstumhormonmangel bei Hypophysenin- & Antiepileptika \\
suffizienz & Aromatasehemmer \\
Subklinische und manifeste Hyperthyreose & Glitazone \\
Diabetes mellitus Typ 1 und Typ 2 & Glukokortikosteroide \\
Rheumatoide Arthritis & $\begin{array}{l}\text { Protonenpumpenhemmer (v. a. in der Langzeitthe- } \\
\text { rapie) }\end{array}$ \\
Spondylitis ankylosans & Schleifendiuretika \\
Billroth-II-Magenresektion oder Gastrektomie & Sturzbegünsigende Medikamente (z. B. Sedativa, \\
Epilepsie & Neuroleptika) \\
Herzinsuffizienz & \\
Zöliakie & \\
Monoklonale Gammopathie unklarer Sginifi- & \\
kanz &
\end{tabular}

Unter der Form der sekundären Osteoporosen zeigt sich ein besonders hoher Anteil an Männer und prämenopausalen Frauen (Bartl und Bartl 2011; NIH 2015).

\subsubsection{Prävention und Therapie der Osteoporose nach Empfehlungen des DVO}

Ziel der präventiven und therapeutischen Maßnahmen ist, das Auftreten der Krankheit zu verhindern oder den Krankheitsprozess bestmöglich einzudämmen.

\subsubsection{Prävention}

Die Initiierung präventiver Maßnahmen wird allen Patienten empfohlen, die aufgrund ihres individuellen Risikoprofils bereits eine Prädisposition haben im Laufe ihres Lebens an einer Osteoporose zu erkranken. Ratsam ist dies laut der Leitlinien des Dachverbandes Osteologie (DVO) demnach für alle Patienten, die bislang noch ein unter 20\%iges 10-Jahres-Frakturrisiko haben.

Als präventive Maßnahmen werden verschiedene Lifestyle- und Ernährungsempfehlungen gegeben. Als eine entscheidende Maßnahme wird die Minimierung von Risikofaktoren genannt, die eine osteoporotische Erkrankung begünstigen. Dazu zählen der Verzicht auf Nikotin und größere Mengen an Alkohol sowie die Überprüfung der regelmäßig einzunehmenden Medikamente und gegebenenfalls die Umstellung auf andere Präparate. Basierend auf der geringen Sensitivität wird ein breitgefächertes Screening auf Osteoporose anhand von Knochedichtemessungen nicht empfohlen. Hinzu kommt, dass eine innerhalb der Normvariation liegende Knochendichte kein Ausschlusskriterium für eine Osteoporose darstellt, da sie weitere 
osteoporosebegünstigende Faktoren außer Acht lässt (Leitlinie Osteoporose 2014; Leitlinie Osteoporose 2017; WHO 2003).

\subsubsection{Spezielle antiosteoporotische Therapieoptionen bei primärer Osteoporose}

Die spezielle antiosteoporotische Therapie bei der primären Osteoporose soll den Krankheitsprogress einschränken. Sie wird basierend auf den DVO-Leitlinien 2017 Patienten empfohlen, die ausgehende von ihrem individuellen Risikoprofil und der DXA-Messung ein über 30\%iges Risiko haben in den nächsten zehn Jahren eine Fraktur zu erleiden. Für Therapieempfehlungen der sekundären Osteoporosen wird auf die entsprechenden fachspezifischen Leitlinien verwiesen (Leitlinie Osteoporose 2017). Zur Therapie der primären Osteoporose bei postmenopausalen Frauen werden laut DVO-Leitlinien momentan sechs Stoffgruppen empfohlen: Bisphosphonate (Alendronat, Risedronat, Ibandronat, Zoledronat), RANKL-Inhibitoren (Denosumab), Östrogene, Östrogen-Rezeptor-Modulatoren (Raloxifen, Bazedoxifen), Teriparatid und Calcitonin als Reservepräparat (Leitlinie Osteoporose 2017). Für alle aufgeführten Präparate besteht eine Therapieempfehlung Evidenzgrad A (nach SIGN-Kriterien) in Bezug auf die Senkung von Wirbelkörperfrakturen. Für Alendronat, Denosumab, Risedronat, Zoledronat, Östrogene besteht zusätzlich eine Empfehlung Evidenzgrad A (nach SIGN-Kriterien) zur Senkung peripherer Frakturen, sowie proximalen Femurfrakturen (Leitlinie Osteoporose 2017).

Für alle aufgeführten Präparate ist eine Reduktion der Frakturhäufigkeiten in einem Zeitraum von drei bis fünf Jahren nachgewiesen. Bisphosphonate und der RANKL-Inhibitor Denosumab können unter optimalen Therapiebedingungen das Frakturrisiko um über $50 \%$ senken. Die Risikoreduktion liegt in Abhängigkeit vom gewählten Präparat zwischen 11\% und 69\% (Aggarwal und Masuda 2018; Siggelkow und Hadji 2016; Tosteson et al. 2008). Als therapeutische Optionen für den Mann sind Alendronat, Risedronat und Zoledronat aus der Stoffgruppe der Bisphosphonate zugelassen sowie Teriparatid und Denosumab (Leitlinienreport Osteoporose 2017). Zur Evaluation der Wirksamkeit einer osteoporosespezifischen Therapie und Abschätzung des absoluten individuellen Frakturrisikos kann die DXA-Messung eingesetzt werden. Demnach erhöht sich das relative Frakturrisiko um den Faktor 1,5, für proximale Femurfrakturen um den Faktor 2,5, wenn der T-Score sich um 1 erniedrigt (Gluer et al. 2004).

\subsection{Osteoporose bei Männern}

Der Großteil der Studien befasst sich, begründet in der höheren Prävalenz, mit der Osteoporose bei Frauen. Aktuelle Zahlen zeigen jedoch, dass in den kommenden Jahren mit einem enormen Anstieg derjenigen Männer zu rechnen ist, die an einer Osteoporose erkranken werden (Marques et al. 2017).Bereits im Jahre 2000 traten von den neun Millionen registrierten Frakturen, die mit einer Osteoporose in Verbindung stehen, 39\% der Frakturen bei Männern auf (Johnell und Kanis 2006). Jeder achte Mann nach dem 50. Lebensjahr wird eine 
osteoporoseassoziierte Fraktur im Laufe seines Lebens erleiden (Khosla et al. 2008). Somit ist das Risiko für Männer, an einer Osteoporose zu erkranken, bereits jetzt schon höher als das Risiko an einem Prostatakarzinom zu erkranken (Jain et al. 2016).

Nur in jungen Jahren (bis ca. zu einem Alter von 45 Jahren) übersteigen die absoluten Frakturzahlen der Männer diejenigen der Frauen. In diesem Alter treten Frakturen bei Männern hauptsächlich im Kontext von hohen traumatischen Ereignissen auf und stehen folglich nicht im Zusammenhang mit pathophysiologischen Veränderungen der Knochenstruktur (Khosla et al. 2008). Zeitlebens höher ist bei Männern die mit osteoporotischen Frakturen assoziierte Morbidität und Mortalität (Flais et al. 2017; Forsen et al. 1999; NIH 2015). Sie versterben nach einer Hüftfraktur doppelt so häufig wie Frauen bereits im Krankenhaus (Khosla et al. 2008). Aus der Dubbo Osteoporosis Epidemiology- Studie geht hervor, dass 39\% der Frauen und $51 \%$ der Männer in den ersten fünf Jahren nach einer initialen osteoporoseassoziierten Fraktur versterben (Bliuc et al. 2013).

\subsubsection{Primäre und sekundäre Osteoporose beim Mann}

Männer verlieren im Zuge der Altersprozesse geschätzt 1\% der Gesamtknochendichte pro Jahr (Hannan et al. 2000). Insgesamt betrachtet ist der Verlust an Knochenmasse bei Männern geringer ausgeprägt als bei Frauen (Khosla et al. 2008). Im Vergleich zu Frauen beginnt der Knochenabbau bei Männern zu einem späteren Zeitpunkt (etwa nach dem 60. Lebensjahr) und verläuft sowohl langsamer als auch kontinuierlicher. Dies steht in Verbindung mit dem weniger abrupten Abfall der männlichen Geschlechtshormone im Alter im Vergleich zu dem postmenopausalen Abfall der weiblichen Geschlechtshormone (Rochira et al. 2006). Studien zeigen, dass auch die Östrogenspiegel der Männer im Alter abfallen. So liegen die gemessenen Werte bei Männer nach dem 60. Lebensjahr im Schnitt um 30\% unter denen von unter 30-jährigen männlichen Patienten (Jockenhövel und Lehnert 2004). Niedrige Östrogenspiegel bei Männern können als ein Prädikator für den Verlust an Knochenmasse fungieren und zudem mit einem erhöhten Risiko für vertebrale Frakturen assoziiert sein (Khosla et al. 2008).

Der Großteil - ca. 85\% - der männlichen Östrogene entsteht in der Peripherie. Vor allem im subkutanen abdominellen Fettgewebe, aber auch im Gehirn, der Prostata und den Knochen finden sich Aromatasen. Sie können lokal Testosteron in Östrogen umwandeln (Gennari et al. 2004). Testosteron kann demnach als ein Vorläuferhormon für das Östrogen angesehen werden. Bei Patienten mit einem kompletten Aromatasemangel entfällt dieser Umwandlungsschritt des Testosterons zu Östrogen und es zeigt sich eine enorme Störung des Knochenstoffwechsels. Diese Männer zeigen das Vollbild einer klinisch manifesten Osteoporose breites vor dem 30. Lebensjahr (Jockenhövel und Lehnert 2004). Auch bei Männern hat Östrogen laut Studien mit ca. 70\% (im Vergleich zu Testosteron mit ca. 30\%) den dominanten Einfluss auf den 
Knochenstoffwechsel. Männer mit niedrigem Testosteron- und niedrigem Östrogenspiegel weisen die höchste Rate an Hüftfrakturen auf (Amin et al. 2006; Khosla 2015).

ImVergleich zu weiblichen Patienten findet sich bei den männlichen Patienten ein deutlich höherer Anteil an sekundären Osteoporosen. In Abhängigkeit von der zugrundeliegenden Studie kann er Werte von bis zu 40\% annehmen. Teils wird dieser hohe Prozentsatz darin begründet, dass die Osteoporose bei Männern immer noch als Rarität angesehen wird und aus diesem Grund eine umfassende Diagnostik durchgeführt wird, ehe die abschließende Diagnose gestellt wird (Khosla et al. 2008; NIH 2015).

Zu den drei häufigsten Ursachen einer sekundären Osteoporose beim Mann zählen: Alkoholabusus, die Einnahme von Glukokortikosteroiden, sowie Hypogonadismus bzw. Hypogonadismus im Kontext eines Klinefelter-Syndroms, einer Hämochromatose eines Hypopituitarismus oder einer hormonell suppressive Therapie zur Behandlung eines Prostatakarzinoms (Smith 2007; Tu et al. 2018). Weitere sekundäre Osteoporosen, in deren Rahmen ein Hypogonadismus auftreten kann sind: Hyperthyreose, Leberzirrhose und verschiedene Medikamente wie beispielsweise Antiepileptika und Antidepressiva (Henzen 2013).

\subsubsection{Spezielle Therapie der Osteoporose beim Mann}

Für die Therapie der Osteoporose beim Mann sind weitaus weniger Präparate zugelassen als zur Therapie der Osteoporose bei der Frau. Zu den gängigen, durch Studien in ihrer Effektivität belegten Präparaten zählen Aledronat, Risedronat, Zoledronat, Teriparatid und Denosumab (Leitlinie Osteoporose 2017; Khosla et al. 2008).

Trotz der hohen Rate an Mortalität und Morbidität, die mit osteoporotischen Frakturen bei Männern assoziiert ist, haben Studien große Lücken in der zielgerichteten osteoporosespezifischen Therapie gezeigt. Demnach wurde nur 7\% der Männer, die eine Hüftfraktur im Alter erlitten, eine antiosteoporotische Therapie empfohlen. Demgegenüber stehen $31 \%$ an Frauen, denen bei gleicher Klinik eine Therapie empfohlen wurde. Fünf Jahre später erhielten sogar $71 \%$ dieser Patientinnen eine spezifische antiosteoporotische Therapie, aber wiederum nur 27\% der Männer. Von diesen 27\% an therapierten Männern, wurden 67\% ein Calcium oder Vitamin D Präparat verschrieben und keines der für die spezielle antiosteoporotische Therapie bei Männern zugelassenes Medikament (Flais et al. 2017; Kiebzak et al. 2002).

\subsection{Nationale und internationale Scores zur Evaluation des osteoporoseassoziierten Frakturrisikos}

Im Folgenden wird auf eine Auswahl nationaler und internationaler etablierter Scores zur Evaluation des osteoporoseassoziierten Frakturrisikos eingegangen. 


\subsubsection{DVO}

Die Leitlinien des DVO fungieren als evidenzbasierte Schlüsselempfehlung für die Primär-, Sekundär- und Tertiärprävention osteoporoseassoziierter Frakturen. Sie sollen die Versorgungsabläufe optimieren und dadurch das Frakturrisiko reduzieren, sowie die Lebensqualität der Patienten verbessern. Sie wurden erstmals im Jahre 2003 herausgegeben und liegen nun in der überarbeiteten Fassung aus dem Jahre 2018 vor. Darin wird empfohlen osteoporosespezifische diagnostische Schritte einzuleiten, sofern das 10-Jahres-Frakurrisiko für radiographische Wirbelkörperfrakturen und proximale Femurfrakturen bei $20 \%$ oder höher liegt. Gültig sind diese Leitlinien für alle postmenopausalen Frauen und alle Männer ab dem 60. Lebensjahr (Leitlinie Osteoporose 2014; Leitlinienreport Osteoporose 2017). Anwendung finden diese Empfehlungen hauptsächlich im deutschsprachigen Raum (Rendl et al. 2013).

\subsubsection{DVO-Leitlinien 2014}

Ausgangspunkt sind eine ausführliche Anamnese und körperliche Untersuchung. Basierend auf diesen Befunden können weitere gezielte Untersuchungen, wie beispielweise eine Knochendichtemessung oder laborchemische Blutuntersuchungen ergänzt werden. Die Anamnese konzentriert sich auf mögliche „red flags“, die mit einem erhöhten Risiko einer osteoporotischen Erkrankung einhergehen. Dargestellt sind diese Risikofaktoren in der Tabelle 3, gruppiert nach Alter jeweils für Männer und Frauen. Ab einem Alter über 70 Jahren bei Frauen und über 80 Jahren bei Männern wird eine generelle Basisdiagnostik empfohlen. Ab diesen Altersklassen gilt das Lebensalter alleinig betrachtet als dominanter Risikofaktor für Frakturen auch ohne weitere klinische Risikofaktoren. 
Tabelle 3: Indikatoren für die Initiierung einer osteoporosespezifischen Diagnostik (basierend auf Siggelkow und Hadji 2016)

\begin{tabular}{|c|c|c|c|c|}
\hline $\begin{array}{l}\text { Indikation zur Basisdiagnostik anhand der DVO-Leitlinie } 2014 \text { zur Pro- } \\
\text { phylaxe, Diagnostik und Therapie der Osteoporose bei postmenopausa- } \\
\text { len Frauen und bei Männern ab dem } 60 \text {. Lebensjahr }\end{array}$ & 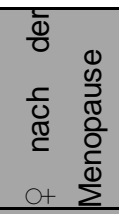 & $\begin{array}{l}8 \\
8 \\
\varepsilon \\
0 \\
0 \\
0 \\
0 \\
0 \\
0\end{array}$ & 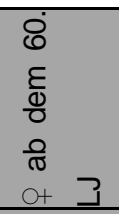 & $\begin{array}{l}0 \\
0 \\
\frac{E}{0} \\
0 \\
0 \\
0 \\
0 \\
0\end{array}$ \\
\hline Niedrigtraumatische singuläre Wirbelkörperfraktur 2. - 3. Grades *2) & $\mathrm{X}$ & $\mathrm{X}$ & $\mathrm{X}$ & $\mathrm{X}$ \\
\hline $\begin{array}{l}\text { Niedrigtraumatische singuläre klinisch manifeste Wirbelkörperfraktur } 1 . \\
\text { Grades mit Deckplattenimpression*2) }\end{array}$ & $\mathrm{X}$ & $\mathrm{X}$ & $\mathrm{X}$ & $\mathrm{X}$ \\
\hline $\begin{array}{l}\text { Niedrigtraumatische singuläre Wirbelkörperfraktur 1. Grades mit Deck- } \\
\text { platten-impression ohne Klinik als Einzelfallentscheidung *1,2) }\end{array}$ & $x$ & $\mathrm{X}$ & $\mathrm{X}$ & $\mathrm{X}$ \\
\hline Niedrigtraumatische multiple Wirbelkörperfrakturen 1.-3.Grades *2) & $\mathrm{X}$ & $\mathrm{X}$ & $\mathrm{X}$ & $\mathrm{X}$ \\
\hline $\begin{array}{l}\text { Niedrigtraumatische nichtvertebrale Frakturen mit Ausnahme von Finger-, } \\
\text { Zehen-, Schädel- u. Knöchelfrakturen }\end{array}$ & $\mathrm{X}$ & $\mathrm{X}$ & $\mathrm{X}$ & $\mathrm{X}$ \\
\hline Cushing Syndrom oder subklinischer Hyperkortisolismus ${ }^{* 4)}$ & $\mathrm{X}$ & $\mathrm{X}$ & $\mathrm{X}$ & $\mathrm{X}$ \\
\hline Primärer Hyperparathyreoidismus $\left.{ }^{4}\right)$ & $\mathrm{X}$ & $\mathrm{X}$ & $\mathrm{X}$ & $\mathrm{X}$ \\
\hline Wachstumshormonmangel *4) & $\bar{X}$ & $\mathrm{X}$ & $\mathrm{X}$ & $\mathrm{X}$ \\
\hline $\begin{array}{l}\text { Bestehende oder geplante orale Glukokortikoidtherapie mit } \geq 2,5 \mathrm{mg} \\
\text { Prednisolonäquivalent tgl. für mehr als } 3 \text { Monate im Jahr *4) }\end{array}$ & $x$ & $\mathrm{X}$ & $\mathrm{X}$ & $x$ \\
\hline Epilepsie / Antiepileptika *4) & $\mathrm{X}$ & $\mathrm{X}$ & $\mathrm{X}$ & $\mathrm{X}$ \\
\hline Monoklonale Gammopathie unklarer Signifikanz & $\mathrm{X}$ & $\mathrm{X}$ & $\mathrm{X}$ & $\mathrm{X}$ \\
\hline Rheumatoide Arthritis & $\bar{X}$ & $\mathrm{X}$ & $\mathrm{X}$ & $\mathrm{X}$ \\
\hline Diabetes mellitus Typ 1 & $\mathrm{X}$ & $\mathrm{X}$ & $\mathrm{X}$ & $\mathrm{X}$ \\
\hline Zustand nach B-II-Operation oder Gastrektomie & $\mathrm{X}$ & $\mathrm{X}$ & $\mathrm{X}$ & $\mathrm{X}$ \\
\hline Spondylitis ankylosans als Einzelfallentscheidung ${ }^{* 1)}$ & $\mathrm{X}$ & $\mathrm{X}$ & & \\
\hline Therapie mit Aromatasehemmern als Einzelfallentscheidung *1)4) & $\mathrm{X}$ & & & \\
\hline Therapie mit Aromatasehemmern *3) 4) & & & $\mathrm{X}$ & \\
\hline Glukokortikoide hochdosiert inhalativ *3)4) & & & $\mathrm{X}$ & $\mathrm{X}$ \\
\hline $\begin{array}{l}\text { Hormonablative Therapie oder Hypogonadismus beim Mann als Einzel- } \\
\text { fallentscheidung }{ }^{* 114)}\end{array}$ & & $\mathrm{X}$ & & \\
\hline Hormonablative Therapie oder Hypogonadismus beim Mann *3)4) & & & & $\mathrm{X}$ \\
\hline Therapie mit Glitazonen *3)4) & & & $\mathrm{X}$ & $\mathrm{X}$ \\
\hline Diabetes mellitus Typ 2 *3) & & & $\mathrm{X}$ & $\mathrm{X}$ \\
\hline
\end{tabular}




\begin{tabular}{|c|c|c|}
\hline Spondylitis ankylosans *3) & $\mathrm{x}$ & $\mathrm{X}$ \\
\hline Proximale Femurfraktur eines Elternteils ${ }^{* 3 \text { ) }}$ & $\mathrm{x}$ & $\mathrm{x}$ \\
\hline Multiple intrinsische Stürze oder erhöhte Sturzneigung ${ }^{\star 3 / 4)}$ & $\mathrm{x}$ & $\mathrm{x}$ \\
\hline Immobilität *3/4) & $\mathrm{X}$ & $\mathrm{X}$ \\
\hline Untergewicht $\left(\mathrm{BMI}<20 \mathrm{~kg} / \mathrm{m}^{2}\right)^{\star 3 / 4)}$ & $\mathrm{x}$ & $\mathrm{x}$ \\
\hline Nikotinkonsum und / oder COPD *3/4) & $\mathrm{X}$ & $\mathrm{X}$ \\
\hline Depression / Antidepressiva *3)4) & $\mathrm{x}$ & $\mathrm{x}$ \\
\hline Hyperthyreose oder subklinische Hyperthyreose, sofern persistierend ${ }^{* 3) 4 \text { ) }}$ & $\mathrm{x}$ & $\mathrm{X}$ \\
\hline Zöliakie *3) & $\mathrm{x}$ & $\mathrm{x}$ \\
\hline Protonenpumpeninhibitoren bei chronischer Einnahme ${ }^{* 3 / 4)}$ & $\mathrm{X}$ & $\mathrm{X}$ \\
\hline Herzinsuffizienz *3)4) & $\mathrm{x}$ & $\mathrm{x}$ \\
\hline 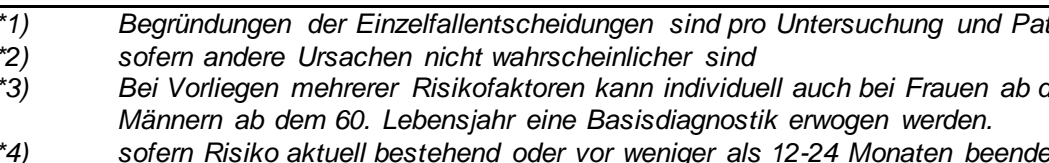 & & \\
\hline
\end{tabular}

Liegen bereits klinische Zeichen von Wirbelkörperfrakturen bzw. proximalen Femurfrakturen vor, kann in Abhängigkeit vom individuellen Risikoprofil auf weitere diagnostische Schritte vor der Therapieeinleitung verzichtet werden. Für alle weiteren Fälle, in denen Risikofaktoren erhoben werden, die auf ein über 20\%iges 10-Jahres-Frakturrisiko hinweisen, wird eine Knochendichtemessung und eine Bildgebung (in der Regel Röntgenuntersuchung) zur Detektion stattgehabter Frakturen empfohlen. Das Standardmessverfahren zur Ermittlung der Knochendichte stellt dabei die DXA-Messung dar. Die Messung sollte dabei an drei Messorten, nämlich der Lendenwirbelsäule, dem Gesamtfemur und dem Femurhals, vorgenommen werden. Bei der Messung an der Lendenwirbelsäule wird der Mittelwert der beurteilbaren Lendenwirbel L1L4 angegeben. Dabei müssen mindestens zwei der vier Wirbel auswertbar sein. Weitere diagnostische Basismaßnahmen umfassen die Überprüfung der Koordinationsfähigkeit und Muskelkraft (beispielsweise durch den Chair-rising-Test, Tandemstand und Timed-up-and-goTest), die Erfassung des Sturzrisikos, der Körpergröße und des Körpergewichts, sowie die Lokalisation von Schmerzen. Darüber hinaus gilt es mögliche Erkrankungen (beispielweise anhand von Laboruntersuchungen) und Medikamente zu erfassen, die eine sekundäre Osteoporose begünstigen können (Niedhart 2016).

Unter Zuhilfenahme der DXA-Messung lässt sich aus der Tabelle 4 ablesen, ob eine medikamentöse Therapie zur Behandlung der Osteoporose indiziert ist. Die Tabelle ist wie folgt zu 
interpretieren: Wird beispielsweise bei einem 82jähringen Mann ein T-Score von -2,4 gemessen, so besteht laut DVO-Leitlinien noch keine Indikation für die Einleitung therapeutischer Maßnahmen. Liegt der gemessene T-Score bei dem gleichen Patienten jedoch bei - 2,6, so wird die Einleitung einer osteoporosespezifischen Therapie empfohlen.

Tabelle 4: Indikation für eine spezifische medikamentöse Therapie (Leitlinie Osteoporose 2014; mit freundlicher Genehmiung durch den DVO)

\begin{tabular}{|c|c|c|c|c|c|c|}
\hline \multicolumn{2}{|c|}{ Lebensalter in Jahren } & \multicolumn{5}{|c|}{$\begin{array}{l}\text { T-Score (Nur anwendbar auf DXA-Werte. Die Wirksamkeit einer } \\
\text { medikamentösen Therapie ist für periphere Frakturen bei einem } \\
\text { T-Score }>-2,0 \text { nicht sicher belegt) }\end{array}$} \\
\hline Frau & Mann ${ }^{2}$ & $-2,0$ bis $-2,5$ & $-2,5$ bis $-3,0$ & $-3,0$ bis $-3,5$ & $-3,5$ bis $-4,0$ & $<-4,0$ \\
\hline $50-60$ & $60-70$ & Nein & Nein & Nein & Nein & Ja \\
\hline $60-65$ & $70-75$ & Nein & Nein & Nein & Ja & Ja \\
\hline $65-70$ & $75-80$ & Nein & Nein & Ja & Ja & $\mathrm{Ja}$ \\
\hline $70-75$ & $80-85$ & Nein & Ja & Ja & $\mathrm{Ja}$ & $\mathrm{Ja}$ \\
\hline$>75$ & $>85$ & Ja & Ja & Ja & Ja & Ja \\
\hline
\end{tabular}

Die Tabelle 4 lässt sich unter Einbeziehung der vom DVO gelisteten Indikationen zur Basisdiagnostik modifizieren. Dem Großteil dieser Faktoren kann ein Wert zwischen 0,5 und 1,0 zugeordnet werden, um den sich die Therapiegrenze in dieser Modellierung erhöht, sofern der entsprechende Risikofaktor vorliegt. Maximal ist eine Anhebung der Therapiegrenze auf einen T-Score von -2,0 möglich, wobei insgesamt nicht mehr als zwei Risikofaktoren berücksichtigt werden sollten. Die einzelnen Risikofaktoren mit ihren dazugehörigen Anpassungsfaktoren sind in der im Anhang im Punkt 6.1 aufgeführt.

Wird so beispielsweise bei einem 71-jährigen Mann ein T-Score von -2,6 gemessen, besteht laut der Tabelle 4 noch keine Empfehlung bezüglich einer osteoporosespezifischen Therapie, sondern erst ab einem T-Score von -3,5. Liegt bei diesem Mann aber zusätzlich ein Nikotinabusus $(+0,5)$ und eine Einnahme von Protoneninhibitoren $(+0,5)$ vor, so erhöht sich die Therapiegrenze um den Betrag von 1,0. Die modifizierte Therapiegrenze, ab der eine spezifische antiosteoporotische Therapie diesem Patienten empfohlen wird, liegt demnach bei einem TScore von -2,5. Mit einem gemessenen T-Score von -2,6 liegt der Patient folglich im Bereich der individuell angepassten Therapiegrenze von $-2,5$ und sollte eine spezielle Therapie laut DVO-Leitlinien erhalten. Dieses Beispiel veranschaulicht die Relevanz der Berücksichtigung der Risikofaktoren.

Ausnahmen von dieser Modellierung der Therapiempfehlungsgrenzen ergeben sich für wenige Faktoren. So wird beispielsweise bei einer singulären niedrigtraumatische Wirbelkörperfraktur 
2. oder 3. Grades oder multiplen niedrigtraumatischen Wirbelkörperfrakturen 1.bis 3. Grades, sofern andere Ursachen einer Fraktur nicht wahrscheinlich sind, eine Therapie ab einem gemessene T-Score unter -2,0 an der Lendenwirbelsäule, dem Schenkelhals oder dem Gesamtfemur empfohlen. Unter Berücksichtigung des individuellen Risikoprofils kann eine Therapie bei einem T-Score >-2,0 in Erwägung gezogen werden.

Auch unter geplanter oder bestehender Therapie mit oralen Glukokortikosteroiden in einer Dosierung $\geq 7,5 \mathrm{mg}$ Prednisolonäquivalent über einen Zeitraum von mehr als drei Monaten ergeben sich abweichende Therapiegrenzen. Eine spezielle Therapie ist in diesem Fall ab einem T-Score von $\leq-1,5$ gemessen an der Lendenwirbelsäule, dem Schenkelhals oder dem Gesamtfemur zu initiieren. Unter Einbeziehung der individuellen Risikoprofils ist auch eine Therapie bei einem T-Score über -1,5 möglich. Zählen zu dem individuellen Risikoprofil niedrigtraumatische Wirbelkörperfrakturen oder multiple periphere Frakturen, so ist eine antiosteoporotische Therapie auch unabhängig von dem ermittelten T-Score indiziert. (Leitlinie Osteoporose 2014).

\subsubsection{DVO-Leitlinien 2017}

Die DVO-Leitlinien 2017 stellen eine überarbeite Version der DVO-Leitlinien 2014 dar. Sie wurden am 26.01.2018 verabschiedet. Erstmals wurden sie nach dem so genannten „Deutschen Instrument zur methodischen Leitlinien-Bewertung („Delbi“) entwickelt. Diese Leitlinien gehen somit zurück auf einer systematische Literaturrecherche (im Zeitraum von 01.01.2013 - 23.05.2016) basierend auf den SIGN (Scottish Intercollegiate Guidelines Network)-Leitlinien und einem interdisziplinären internen und externen Konsensus Prozess. Die Literaturrecherche wurde in der Datenbank der Arbeitsgemeinschaft der Wissenschaftlichen Medizinischen Fachgesellschaften (AWMF) und des Guidelines International Network (G-I-N) durchgeführt. Sie konzentrierte sich auf 17 Schlüsselfragen, die im Vorfeld durch Arbeitsgruppen ausgearbeitet wurde. Die Auswertung umfasst insgesamt 52 deutsch- und englischsprachige Leitlinien. Die draus resultierenden Ergebnisse wurden in die DVO-Leitlinien 2014 eingearbeitet bzw. ergänzt (Leitlinienreport Osteoporose 2017).

Neuerungen in den DVO-Leitlinien 2017 zeigten sich in der Auswahl der Risikofaktoren, der laborchemischen Basisdiagnostik und der Therapieempfehlungen (bereits unter 1.1.3 und 1.2.2 aufgeführt). Die DVO-Leitlinien 2017 wurden um die folgenden Risikofaktoren ergänzt: systemischer Lupus Erythematodes, Schizophrenie, apoplektischer Insult, Alzheimer Erkrankung, Morbus Parkinson, Alkohol und alkoholische Lebererkrankung, Anorexia nervosa und Opioide. Für die folgenden Risikofaktoren verschob sich im Vergleich zu den Leitlinien 2014 das Alter, ab dem eine Basisdiagnostik empfohlen wird, von 60 auf 50 Jahre bei Frauen bzw. von 70 auf 60 Jahre bei Männern: Subklinische und manifeste Hyperthyreose, Diabetes mellitus Typ 2, Spondylitis ankylosans, Zöliakie, Depressionen, Herzinsuffizienz, hochdosierte 
Glukokortikoidtherapie inhalativ, Therapie mit Thiazolidindionen und Protonenpumpenhemmer bei chronischer Einnahme.

Das Basislabor wurde in den DVO-Leitlinien 2017 um die folgenden Parameter ergänzt: Kalziumausscheidung im 24-h Urin (bei V.a. idiopathische Hyerkalzurie), Kadmiumbestimmung im Urin (bei V.a. erhöhte Kadmiumexposition), sowie ggf. eine Ergänzung der SerumeiweiBelektrophorese um eine Immunfixation.

\subsubsection{FRAX}

Der FRAX-Algorithmus zur Evaluation des 10-Jahres-Frakturrisikos wurde im Jahre 2008 publiziert und steht mittlerweile für 63 Länder zur Verfügung und ist somit für $80 \%$ der Gesamtweltbevölkerung verfügbar (Oden et al. 2015). Zur Modellierung wurden Patientendaten aus neun Kohorten evaluiert. Validiert wurde er in 11 unabhängigen prospektiven Studien, die insgesamt über 1 Millionen Patienten umfassten (Kanis et al. 2013; Leslie et al. 2010). Der FRAX-Rechner integriert ein festes Set an Risikofaktoren (genaue Auflistung unter 3.5.3). Jedem einzelnen Faktor kommt dabei eine Gewichtung entsprechend seinem Einfluss auf die Entstehung osteoporoseassoziierter Frakturen zu. Bei dieser Berechnung wird eine Interaktion der Faktoren untereinander berücksichtigt, die genaue Interaktion der Faktoren im FRAX ist jedoch nicht bekannt.

Das Ergebnis des internet- bzw. des vereinfachten papierbasierten FRAX-Rechners wird in Form von zwei 10-Jahres-Frakturwahrscheinlichkeiten („major osteoporotic“ Fraktur (MOF) und Hüftfraktur (HF)) angegeben. Zu den MOFs zählen Hüft-, Wirbelkörper-, Schulter- und Handgelenksfraktur (Kanis et al. 2008). Der FRAX-Rechner liefert prozentuale Werte zur Frakturrisikoprognose, spricht aber keine Empfehlungen bezüglich der Unterlassung oder Initiierung einer osteoporosespezifischen Therapie aus. Für Therapieentscheidungen verweist er auf die landesspezifischen Leitlinien zur Osteoporosetherapie. In einer Zusammenschau verschiedener länderspezifischer Leitlinien und wissenschaftlicher Arbeiten wird ein Risiko $\geq 20 \%$ für eine MOF bzw. $\geq 3 \%$ für eine $\mathrm{HF}$ als die am häufigsten angewandte Interventionsgrenze beschrieben (Kanis et al. 2016a). Die deutschen DVO-Leitlinien empfehlen die Initiierung einer spezifischen antiosteoporotischen Therapie ab einem anhand des FRAX-Rechners ermittelten 10-Jahres-Frakturrisiko von $\geq 14 \%$ für eine MOF und $\geq 3 \%$ bei einer HF(Leitlinie Osteoporose 2017). 


\subsubsection{GARVAN-Score}

Der internetbasierte GARVAN-Rechner (Eingabemaske in der Abbildung 1) basiert auf Daten aus der „Dubbo Osteoporosis Epidemiology“ Studie (Bolland et al. 2011). Das Modell errechnet jeweils prozentuale Frakturrisiken für die nächsten fünf bzw. zehn Jahre und bezieht sich dabei auf das Auftreten von Schenkelhals- und „any osteoporotic“ Frakturen. Unter den Begriff „any osteoporotic“ Frakturen fallen Schenkelhals-, symptomatische Wirbelkörper-, Unterarm-, Mittelhand-, Oberarm-, Schulterblatt-, Schlüsselbein-, distale Femur-, proximale Tibia-, Patella-, Becken- und Sternumfrakturen (Sandhu et al. 2010). Der GARVAN-Score wird hauptsächlich in Australien verwendet (Aspray 2015).

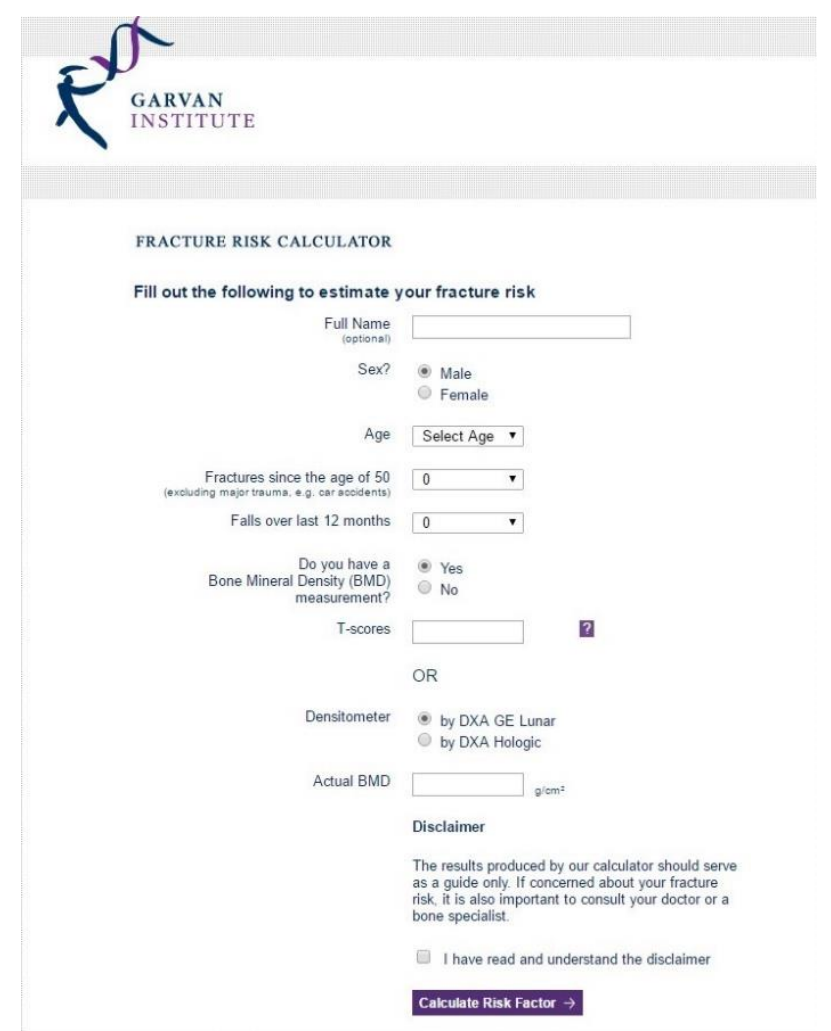

Abbildung 1: GARVAN-Eingabemaske (http://www.garvan.org.au/promotions/bone-fracture-risk/calculator/, Zugriff am: 02.11.2017)

In den GARVAN-Risikoscore werden fünf Risikofaktoren integriert: Alter, Geschlecht, Anzahl der Stürze im letzten Jahr, Anzahl der Frakturen nach dem 50. Lebensjahr und der am Schenkelhals gemessene T-Score bzw. die am Schenkelhals gemessene Knochendichte oder alternativ das Gewicht. Um den GARVAN-Score anwenden zu können, muss das Alter zwischen 50 und 96 Jahren liegen. Anderenfalls sind nur näherungsweise Berechnungen möglich. In Bezug auf die stattgehabten Stürze im letzten Jahr und den Frakturen nach dem 50 . Lebensjahr werden definitive Zahlenwerte von null bis drei zur Auswahl vorgegeben, höhere Werte werden in die Option „drei und mehr" eingeschlossen.

Bei der Eingabe bezüglich der Knochendichte stehen mehrere Optionen zur Verfügung. Es besteht die Auswahlmöglichkeit zwischen der Angabe der gemessenen Knochendichte in 
$\mathrm{g} / \mathrm{cm}^{2}$ oder des T-Scores. Angaben zur Knochendichte bzw. der T-Score sind nicht zwingend notwendig für die Modellierung. Liegen diese nicht vor, so wird alternativ das Gewicht in das Modell miteinbezogen.

Zusätzlich zu den prozentualen Frakturrisiken generiert der Rechner eine Aussage, ab welchem Risiko eine pharmakologische osteoporosespezifische Therapie einen positiven Effekt hat. Zur Minimierung des Auftretens einer Schenkelhalsfraktur ist es demnach empfehlenswert eine Therapie einzuleiten, wenn das 5-Jahres-Frakturrisiko zwischen $2 \%$ und 5\% liegt bzw. das 10-Jahres-Frakturrisiko zwischen 3\% und 9\%. Bezieht man diese Therapiegrenzen auf „any osteoporotic/fragility“ Frakturen, so geht man davon aus, dass ab einem 5-Jahres-Frakturrisiko von $8 \%$ bis $13 \%$ und einem 10 -Jahres-Frakturrisiko von $14 \%$ bis $26 \%$ die Initiierung therapeutischer Maßnahmen einen positiven Effekt hat.

\subsubsection{QFracture ${ }^{\circledR}$}

Der QFracture $\AA^{\circledR}$ Risikorechner findet auf Empfehlung des „National Institute for Health and Care Excellence" (NICE) vorrangig Anwendung in England und Wales. Er basiert auf Daten von über zwei Millionen britischen Patienten (zwischen 30 und 85 Jahren), die im Rahmen der ärztlichen Grundversorgung erhoben wurden. Er dient der Ermittlung des Frakturrisikos. Wahlweise kann das Risiko zwischen ein bis zehn Jahre ermittelt werden (Aspray 2015). Dabei wird jeweils ein prozentuales Risiko für die Wahrscheinlichkeit einer Hüftfraktur und „any osteoporotic“ Fraktur (Hüft-, Handgelenks-, Schulter- und Wirbelsäulenfrakturen) errechnet. Eine Therapieempfehlung wird nicht ausgesprochen. Das Scottish Intercollegiate Guidelines Network (SIGN) empfiehlt jedoch weitere diagnostische Schritte einzuleiten, sofern ein Frakturrisiko über 10\% ermittelt wird (Kanis et al. 2016b).
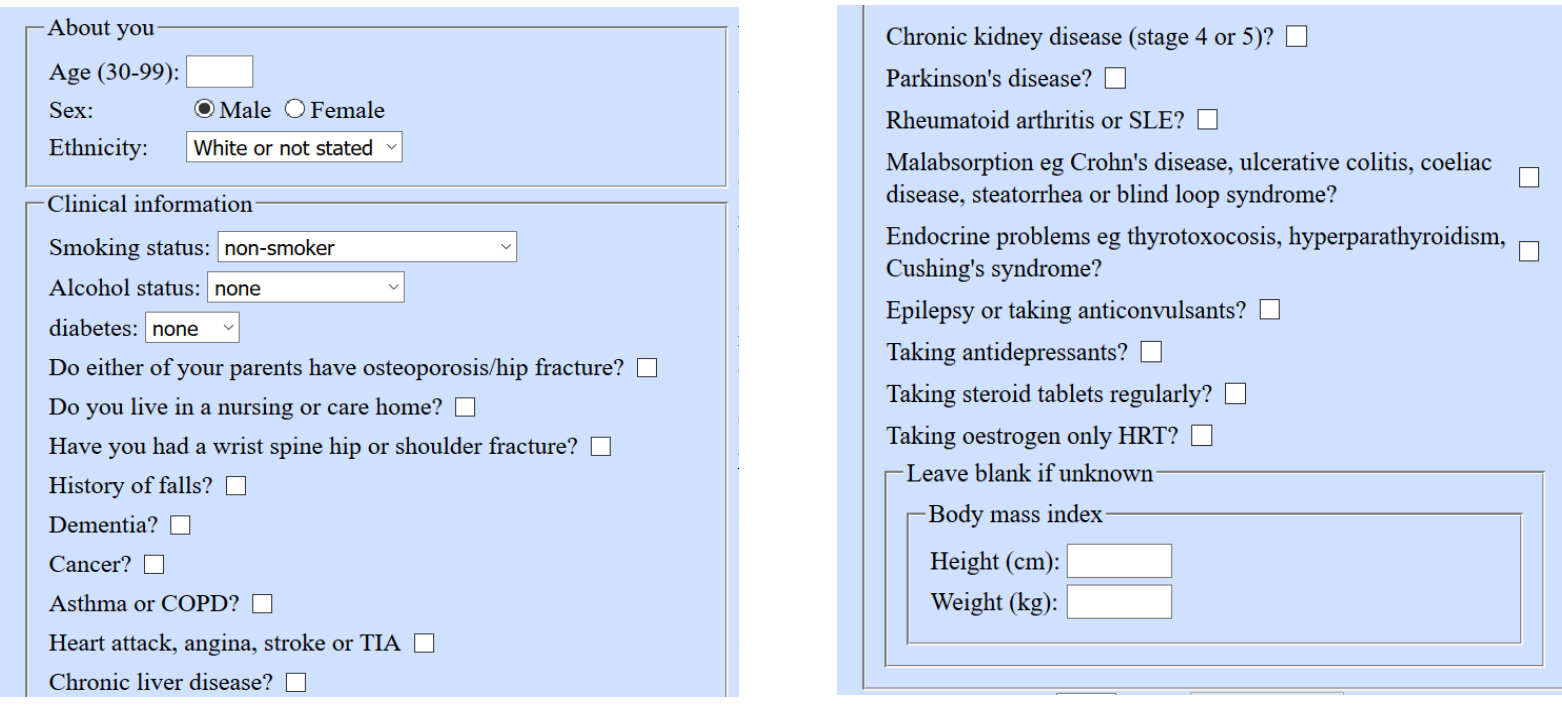

Abbildung 2: QFracture® Eingabemaske (http:/www.qfracture.org/, Zugriff am 02.11.2017) 

Anwendbar ist der QFracture® Risikorechner für Patienten zwischen 30 und 99 Jahren. Er erfordert keine labormesstechnischen Parameter und auch keine Knochendichtemessung, weshalb er gut zur Selbstabschätzung des Frakturrisikos genutzt werden kann. Eine Darstellung der verwendeten Eingabemaske zeigt die Abbildung 2. Ein besonderes Merkmal des QFracture ${ }^{\circledR}$ Risikorechner ist es, dass er insgesamt 26 Faktoren in die Risikoevaluation einbezieht. Dabei besteht bei den Faktoren Tabak- und Alkoholkonsum die Möglichkeit einer genaueren Spezifizierung bezüglich der zu sich genommenen Mengen. 


\section{Zielsetzung}

Ziel dieser Arbeit ist es, ein Kollektiv männlicher Patienten, die im MVZ endokrinologikum Göttingen zwischen Juli 2007 und Juni 2014 vorstellig waren, bezüglich osteoporosespezifischer Merkmale zu untersuchen. Anhand der vorliegenden osteoporosespezifischen Daten werden für jeden Patienten verschiedene nationale und internationale Scores zur Osteoporoseevaluation angewandt. Anhand der verschiedenen Scores sollen die 10-Jahres-Frakturprognosen und die darauf basierenden Therapieentscheidungen verglichen werden.

Es handelt sich dabei teils um validierte (FRAX-Score, DVO-Score) und nicht validierte Scores (Score nach C. Glüer, „FRAX+“-Score). Die Scores unterscheiden sich bezüglich der integrierten Risikofaktoren. Für den in Deutschland verwendeten DVO-Score werden eindeutige Therapiegrenzen angegeben. Der FRAX-Score dahingegen legt keine Therapiegrenzen fest, es existieren jedoch länderspezifische Therapieempfehlungen. In dieser Arbeit werden für den FRAX-Score Therapiegrenzen verwendet, die sich anhand von Metaanlaysen als die am häufigsten im internationalen Vergleich verwendeten Therapiegrenzen herausgestellt haben. Zudem wird die für Deutschland festgelegte Therapiegrenze im Vergleich dazu betrachtet.

Für den FRAX-Score wird in dieser Arbeit zusätzlich eine Erweiterung des Sets der sekundären Osteoporoseformen vorgenommen (im Weiteren als „FRAX+“-Score bezeichnet). Dadurch soll untersucht werden, inwiefern sich das 10-Jahres-Frakturrisiko unter Erfassung zusätzlicher sekundärer Osteoporoseformen verändert. Durch ein lineares Regressionsmodell wird zudem die Gewichtung der einzelnen im FRAX-Score integrierten Faktoren genauer analysiert.

Im Vordergrund dieser Arbeit stehen die Analyse und der Vergleich der verschiedenen Scores. Eine Aussage über die Genauigkeit der Frakturprognose der jeweiligen Scores kann basierend auf den erhobenen Daten nicht getroffen werden. Um eine diesbezügliche Aussage treffen zu können, wäre eine erneute Patientenbefragung bezüglich stattgehabter Frakturen durchzuführen. 


\section{Material und Methoden}

\subsection{Studiendesign}

Die in dieser Studie durchgeführten Analysen und Auswertungen beruhten auf dem Modell einer retrospektiven Datenanalyse. Alle Patientendaten wurden zwischen dem 01.07.2007 und 30.06.2014 in dem MVZ endokrinologikum Göttingen von den dort tätigen Ärztinnen und Ärzten erhoben und anschließend digital archiviert.

\subsection{Studienkollektiv}

Im Zeitraum vom 01.07.2007 bis 30.06.2014 waren insgesamt 2021 Patienten unter dem Diagnoseschlüssel "Osteoporose“ im medizinischen Versorgungszentrum MVZ endokrinologikum Göttingen vorstellig. Um die Patienten in die retrospektive Studie einzubeziehen, musste eine schriftliche Einwilligung zur Verwendung der patientenbezogenen Daten zu wissenschaftlichen Forschungszwecken vorliegen. Zudem musste Zugriff auf einen Arztbrief und vollständig ausgefüllte Aufnahmeunterlagen bestehen, die nicht deutlich älter als sechs Monate waren. Diese Unterlagen durften nicht vor dem 01.07.2007 oder nach dem 30.06.2014 datiert sein. All diese Kriterien trafen für 147 männliche Patienten zu.

\subsection{Genehmigung durch die Ethikkommission}

Die Genehmigung durch die Ethikkommission Göttingen erfolgte am 18.02.2007 (18/2/07).

\subsection{Datenquellen}

Alle im Kontext der Erstvorstellung im MVZ endokrinologikum Göttingen erhoben Daten wurden systematisch den unterschiedlichen Dokumenten (Arztbriefe, Anamnesebögen Gesprächsnotizen, Laborbefunde, DXA-Messungen, eingescannten Vorbefunde) aus der „Medistar Software" entnommen und in eine Exceltabelle kategorisch überführt. Zur Sicherung der Datenqualität und Fehlerprüfung wurden diese Arbeiten in Kooperation mit Frau Giessel durchgeführt, die ebenfalls als Doktorandin im MVZ endokrinologikum Göttingen tätig war. Als primäre Datenquelle wurden die Arztbriefe verwendet. Die in den Arztbriefen fehlenden Angaben wurden den Patientenfragebögen (siehe Anhang) entnommen. Bei Abweichungen der Angaben in diesen Datenquellen, wurden die Informationen aus dem Arztbrief verwendet.

Alle Daten standen für die Auswertung in digitaler Form zur Verfügung. Sofern nach dem Erstaufnahmegespräch weitere diagnostische Maßnahmen durchgeführt wurden, um die Pathologie der osteoporotischen Erkrankung abzuklären und mögliche Differentialdiagnosen auszuschließen, wurden die Abschlussdiagnose (ob es sich um eine primäre oder sekundäre Osteoporose handelt) nach Zusammenschau aller durchgeführten Untersuchungen aus einem späteren Arztbrief übernommen. 
Die für die Studie relevanten Daten umfassten alle Parameter, die für die Kalkulation des Osteoporoserisikos anhand der DVO Leitlinien, dem internetbasierten FRAX-Rechner und des Risikoscores nach C. Glüer benötigt wurden.

\subsubsection{Anamnesebögen}

Bei Erstaufnahme erhielten alle Patienten die im Anhang 6.2 und 6.3 abgebildeten Fragebögen. Diese wurden selbstständig ausgefüllt, offene Fragen wurden im sich anschließenden Arztgespräch geklärt. Der Fragebogen „Ärztliche Fragen vor der Untersuchung“ war weniger osteoporosespezifisch und wurde von allen Patienten, die im MVZ endokrinologikum Göttingen vorstellig waren, ausgefüllt. Bis auf das Gewicht, die Größe und das Alter handelte es sich bei den erfragten Symptomen um dichotome Fragestellungen, die mit "ja“ oder "nein“ zu beantworten waren. Zudem bestand in ausgewählten Fällen die Möglichkeit die Antwort in Form von Freitext genauer zu spezifizieren.

Der „Osteoporoserisiko-Fragebogen“ beinhaltete Fragestellungen zu osteoporosespezifischen Risikofaktoren und der Knochengesundheit. Teilweise zeigten sich Überschneidungspunkte zwischen den beiden Fragebögen. Wichtige osteoporosespezifische Aspekte waren die Erfassung von:

- der ersten und letzten Regelblutung bei Frauen

- familiärem Auftreten der Osteoporose und damit in Verbindung stehenden Frakturen, im speziellen Schenkelhalsfrakturen

- bestimmten Krankheitsbildern, klinischen Symptomen und Medikamenten, die mit einer primären oder sekundären Osteoporoseformen assoziiert sind

- Rückenschmerzen und deren Charakter

- Lifestylefaktoren, die besonders die Knochengesundheit beeinflussen (Rauchen, Alkohol, Milchkonsum, spezielle Ernährungsgewohnheiten, Mobilität, körperliche Ertüchtigung)

- der Größenabnahme

- des Körpergewichts bzw. einer Gewichtsabnahme in den letzten zwei Jahren

- der Sturzanamnese

- der Frakturanamnese, insbesondere unter dem Aspekt der Frakturgenese (traumatisch oder atraumatisch) und Lokalisation

Auch hier bestanden bei den meisten Fragen die Auswahloptionen „ja“, „nein“ oder „unbekannt“. In Bezug auf die Medikamentenanamnese wurden Medikamente aufgeführt, die in Verbindung mit einer Osteoporose bzw. der Therapie der Osteoporose stehen. Dazu zählen: Cortison, Antiepileptika, Schlafmittel, Marcumar/Sintron, Heparin, Schilddrüsenhormone, Antirheumatika, Calcium, Vitamin D, Calcitonin, Bisphosphonat, Fluor und Hormone. 
Zur genaueren Spezifizierung der Lifestylefaktoren (Zigarettenkonsum, Alkoholkonsum, Diät, Milchkonsum, Käse und Joghurt, Sport und Immobilität) erfolgte eine vierstufige Einteilung (siehe Abbildung 3).

7 - 9 Lebensgewohnheiten:

\begin{tabular}{|l|c|c|c|c|}
\hline Zigarettenkonsum: & keinen & unter 10 Zig. & bis 20 Zig. & über 20 Zig. \\
\hline Alkoholkonsum: & keinen & gelegentlich & bis 2/4 Wein & über 2/4 Wein \\
\hline Diät: & keinen & (fast) vegetarisch & Diabetes & sonstiges \\
\hline Milchkonsum: & keinen & wenig & bis 1/4 tgl. & mehr \\
\hline Käse u. Joghurt: & Keinen & manchmal & öfters & viel \\
\hline Sport: & keinen & fallweise & regelmäßig & viel \\
\hline Immobilität: & nie & 1 Monat & 2 Monate & mehr \\
\hline
\end{tabular}

Abbildung 3: Lebensgewohnheiten im Kontext der osteoporosespezifischen Anamnese (Auszug aus dem Osteoporoserisko-Fragebogen; mit freundlicher Genehmigung durch H. Siggelkow)

\subsubsection{Klinische Untersuchung}

Die ärztlicherseits durchgeführte körperliche Untersuchung beinhaltete sowohl eine grob orientierende Erfassung der allgemeinen gesundheitlichen Konstitution (Größe und Gewicht, die Messung von Blutdruck und Puls, sowie die Auskultation von Herz und Lunge und die Palpation der Schilddrüse), als auch der osteoporosespezifischer Pathologien. Die körperliche Untersuchung konzentrierte sich auf die Identifikation von möglichen charakteristischen Anzeichen einer primären oder sekundären Osteoporose oder differentialdiagnostisch zu betrachtenden Krankheitsbildern sowie die Einschätzung der Funktionseinschränkung und Schmerzcharakteristika. In diesem Rahmen wurde besonders auf akute oder chronische Schmerzen mit damit verbunden Haltungs- und Verhaltensauffälligkeiten, verringerten Finger-Boden-Abstand, Größenabnahme, lokalen Druckschmerz über der Wirbelsäule, pathologischen Schober Test oder positiven Lasègue-Test geachtet. Komplettiert wurde die körperliche Untersuchung durch Tests zur Beurteilung der Muskelkraft, dem Gleichgewichtssinn, der Koordination und Sturzneigung.

\subsubsection{Osteodensitometrie}

Das international anerkannte Verfahren zur Untersuchung der Mikroarchitektur des Knochens und seiner Masse stellt die Osteodensitometrie dar. Sie dient der objektiven osteodensitometrischen Osteoporoseklassifikation und ist unabhängig von klinischen Manifestationen des Krankheitsbildes und anamnestischen Risikofaktoren (Hadji et al. 2002). Sie ist indiziert, wenn aufgrund der anamnestisch erhobenen Daten und der klinischen Symptome ein erhöhtes Osteoporoserisiko vorliegt. Eine Messung ist nur dann durchzuführen, wenn sich aus ihrem Ergebnis eine mögliche therapeutische Konsequenz für den Patienten ergibt.

Zu den international gängigen Verfahren der Osteodensitometrie zählen die Duale Energy Xray Absorptiometry (DXA), die Digital-Röntgen-Radiogrammetrie (DXR), die Quantitative Computertomographie (QCT) und die Quantitative Ultrasonometrie (QUS). Grundprinzip aller 
Verfahren ist eine nicht-invasive Messung des Gehalts an Calciumkristallen im Knochen. Gemessen wird die Absorption der Röntgenstrahlung im Knochengewebe. Daraus ergibt sich die Masse calciumreicher Kristalle pro Volumen Materie (Kann 2001). Die weltweit am meisten genutzte Methode ist DXA. Die Genauigkeit und Aussagekraft der DXA wurde in zahlreichen Studien evaluiert, fehlerhaft Messwerte treten in $5 \%$ bis $8 \%$ der Fälle auf (El Maghraoui und Roux 2008). Dieses Verfahren wurde auch im MVZ endokrinologikum Göttingen angewandt.

Für die Bestimmung der Knochendichte mithilfe der DXA liegen drei gängige Messorte vor: Die lumbale Wirbelsäule im Bereich L1 bis L4, der proximale Femur sowie der Unterarm. Am häufigsten werden die Messungen an der lumbalen Wirbelsäule und dem proximalen Femur durchgeführt (Hans et al. 2006). Obwohl die einzelnen Messorte in ihrer Knochendichte miteinander korrelieren, sind Unstimmigkeiten und abweichende Knochendichtewerte keine Seltenheit (Moayyeri et al. 2005). Zurückzuführen sind diese Variationen auf verschiedene pathophysiologische Prozesse. Die Hauptgründe sind Studien zufolge unterschiedliche physiologische und anatomische bedingte Strukturen sowie mechanische Belastung, sekundäre Veränderungen im Rahmen von ossären Erkrankungen und Messfehler durch Artefakte oder durchführungstechnische Ungenauigkeiten (El Maghraoui et al. 2007). Beobachtungen zeigten, dass sich der Femur (Femurhals, Trochanter, gesamter Femur) am besten zur Vorhersage einer Hüftfraktur eignet, wohingegen die lumbale Wirbelsäule am besten für die Kontrolle des Therapieverlaufs geeignet ist (El Maghraoui und Roux 2008).

Andere Verwahren, wie beispielweise die quantitative Computertomographie (QCT) und der quantitative Ultraschall, eigenen sich ähnlich gut zur Fraktureinschätzung. Für sie existiert aber keine gültigen Richtwerte zur Osteoporoseevaluation basierend auf WHO-Empfehlungen. Auch liegen keine Studien vor, die ein Therapieansprechen bei den durch diese Verfahren ermittelten Frakturrisiken belegen (Gluer et al. 2004).

Je nachdem in welchem Zentrum die Messungen durchgeführt wurden, unterschieden sich die verwendeten Geräte in ihren Herstellern. Im MVZ endokrinologikum Göttingen wurde für die in die Studie eingeschlossenen Patienten das Gerät „GE-Lunar Prodigy“ der Marke „GE Healthcare" verwendet.

\subsubsection{Laboruntersuchung}

Im Rahmen der osteoporosespezifischen Eingangsevaluation wurde für jeden Patienten ein Basislabor abgenommen. Die dabei ausgewerteten Parameter waren Bestandteil der Bewertung des allgemeinen Gesundheitszustandes und der Knochengesundheit der Patienten. Sie gaben Hinweise auf mögliche sekundäre Ursachen der osteoporotischen Erkrankung. 
Im MVZ endokrinologikum Göttigen umfasste die labortechnische Blutuntersuchung standardmäßig das Basislabor nach den Leitlinien des DVO (Serum-Kalzium, Serum-Phosphat, SerumNatrium (optional), Alkalische Phosphatase (Serum), Gamma-GT, Kreatinin-Clearance, BSG/C-Reaktives Protein, Blutbild, Serum-Eiweißelektrophorese evtl. mit Immunfixation, TSH) (Leitlinie Osteoporose 2017). Ergänzend wurden in Abhängigkeit von der Arbeitshypothese folgende Parameter bestimmt: Parathormon (intakt), Cortisol, Estradiol bei Frauen bzw. Testosteron bei Männern, BAP, Osteocalcin, Desoxypyridinolin, Vitamin D, die Kalziumausscheidung im 24-h-Urin oder die Kadmiumbestimmung im Urin.

\subsection{Datenauswertung basierend auf den DVO-Leitlinien}

Die Datenerhebung erstreckte sich über eine Zeitspanne von 2007 bis 2014. In diesem Zeitraum wurden die DVO Leitlinien zweimal aktualisiert. Da der Großteil der Patienten nach den Leitlinien aus dem Jahre 2009 diagnostiziert wurde, werden diese erläutert und die prägnantesten Abweichungen der Leitlinien aus den Jahre 2006 und 2014 aufgezeigt. Auf die Leitlinien aus dem Jahr 2014 wurde bereits detailliert im Punkt 1.3.1.1 eingegangen.

Anhand des vorliegenden Risikoprofils ließen sich die untersuchten Patienten nach den DVOLeitlinien in drei Kategorien einteilen: Patienten mit einem 10-Jahres Frakturrisiko unter 20\%, einem 10-Jahres-Frakturrisiko zwischen 20 und 30\% und einem 10-Jahres-Frakturrisiko über $30 \%$. Ein über $20 \%$ iges 10-Jahres-Frakturrisiko bestand, sofern einer der in der Abbildung 4 gelisteten Risikofaktoren (aufgeteilt nach Geschlecht und Altersgruppe) vorlag. Unabhängig von den vorliegenden Risikofaktoren wurde allen weiblichen Patienten über 70 Jahren bzw. allen männlichen Patienten über 80 Jahren ein über 20\%iges 10-Jahres Frakturrisiko zugeordnet. 


\section{Anamnestische und klinische Risikofaktoren für osteoporotische} Frakturen

Die nachfolgende Tabelle zeigt (jeweils mit , $+^{n}$ gekennzeichnet) die klinischen Risikofaktoren, bei denen in Abhängigkeit von Alter und Geschlecht ein 10-Jahres-Frakturrisiko $>20 \%$ für Wirbelkörperfrakturen und proximale Femurfrakturen vorliegt, bzw. möglich ist oder bei denen sich unmittelbare therapeutische Konsequenzen ergeben (z.B. OP-Indikation beim pHPT). Ab einem Alter von 70 J. bei Frauen und von 80 J. bei Mannern ist das Risiko generell $>20 \%$.

\begin{tabular}{|c|c|c|c|}
\hline Frauen & $<50$ Jahre & 50-60 Jahre & 60-70 Jahre \\
\hline Männer & $<60$ Jahre & 60-70 Jahre & 70-80 Jahre \\
\hline $\begin{array}{l}\text { Singuläre Wirbelkörperfraktur 2.-3. Grades } \\
\text { (d.h. } 25-40 \% \text { bzw. }>40 \% \text { Höhenminderung) }\end{array}$ & $+(\mathrm{D})$ & $+(A)$ & $+(A)$ \\
\hline Multiple Wirbelkörperfrakturen 1.-3. Grades & $+(\mathrm{D})$ & $+(\mathrm{A})$ & $+(\mathrm{A})$ \\
\hline Orale Glukokortikoide $\geq 7,5 \mathrm{mg}$ Prednisolonāquivalent $\geq 3$ Monate* & $+(\mathrm{A})$ & $+(\mathrm{A})$ & $+(\mathrm{A})$ \\
\hline Cushing-Syndrom* & $+(B)$ & $+(B)$ & $+(\mathrm{A})$ \\
\hline Subklinischer Hyperkortisolismus* & $+(\mathrm{D})$ & $+(\mathrm{D})$ & $+(B)$ \\
\hline Primärer Hyperparathyreoidismus (pHPT) ${ }^{*}$ & $+(B)$ & $+(B)$ & $+(B)$ \\
\hline Singuläre Wirbelkörperfraktur 1. Grades (d.h. 20-25\% Hohenminderung) & ** & ** & $+(\mathrm{A})$ \\
\hline Orale Glukokortikoide $<7,5 \mathrm{mg}$ Prednisolonăquivalent $>3$ Monate * & & $+(\mathrm{A})$ & $+(\mathrm{A})$ \\
\hline Therapie mit Glitazonen bei Frauen* & & $+(\mathrm{D})$ & $+(\mathrm{A})$ \\
\hline Wachstumshormonmangel bei Hypophyseninsuffizienz & & $+(\mathrm{B})$ & $+(B)$ \\
\hline Nichtvertebrale Fraktur(en) nach dem 50. Lebensjahr & & ** & $+(\mathrm{A})$ \\
\hline Therapie mit Aromatasehemmerrn* & & *** & $+(\mathrm{A})$ \\
\hline Antiandrogene Therapie ${ }^{*}$ & & *** & $+(A)$ \\
\hline Rheumatoide Arthritis & & ** & $+(\mathrm{A})$ \\
\hline Proximale Femurfraktur eines Elternteils & & & $+(B)$ \\
\hline Untergewicht $(\mathrm{BMI}<20)^{*}$ & & & $+(\mathrm{A})$ \\
\hline Nikotinkonsum* & & & $+(\mathrm{A})$ \\
\hline Multiple Stūrze* & & & $+(\mathrm{A})$ \\
\hline Immobilitāt** & & & $+(A-B)$ \\
\hline Epilepsie / Antiepileptika* & & & $+(\mathrm{A})$ \\
\hline Zustand nach B-II-Operation oder Gastrektomie & & & $+(A)$ \\
\hline Diabetes mellitus Typ 1 & & & $+(\mathrm{A})$ \\
\hline TSH-Werte $<0,3 \mathrm{mU} / \mathbf{1}^{*}$ & & & $+(B)$ \\
\hline $\begin{array}{l}\text { Sturzbegünstigende Medikamente (Sedativa, Orthostase-verursachend, } \\
\text { Antidepressiva)* }\end{array}$ & & & $+(B-D)$ \\
\hline
\end{tabular}

wenn ${ }_{a}+^{n}$ oder wenn Alter $>70$ Jahre Frau $(A)$ oder $>80$ Jahre Mann $(A) \rightarrow$ Indikation zur Basisdiagnostik gegeben (sofern damit verbundene therapeutische Maßnahmen umgesetzt werden können).

AuBerhalb dieser Konstellationen wird, von seltenen Ausnahmen abgesehen, derzeit keine Diagnostik empfohlen (D),

Bei einem Beratungsanlass aufgrund der Erbringung technischer Befunde außerhalb der DVO-Empfehlungen in Form von Knochendichtemess-

verfahren, quantitativem Ultraschall oder Knochenmarkern wird bezüglich der Risikoabschätzung und der evtl. Notwendigkeit der Durchführung

einer Basisdiagnostik auf die Langfassung verwiesen.

Abbildung 4: Anamnestische und klinische Risikofaktoren nach den DVO-Leitlinien 2009 (mit freundlicher Genehmigung durch den DVO)

Lag das 10-Jahres-Frakturrisiko über $20 \%$ so wurde eine Knochendichtemessung mittels DXA als weiterführende osteoporosespezifische Diagnostik empfohlen. Anhand des ermittelten TScores ließ sich entscheiden, ob das 10-Jahres-Frakturrisiko über $30 \%$ lag und somit die Initiierung einer osteoporosespezifischen Therapie empfohlen wurde. Als Entscheidungsgrundlage diente dabei die Tabelle 5. Sie gibt die alters- und geschlechtsspezifischen Untergrenzen gemessener T-Scores an, ab denen eine Therapieempfehlung ausgesprochen werden sollte.

Tabelle 5: Indikationen für eine spezifische medikamentöse Therapie in Abhängigkeit von Geschlecht, Alter und DXA-Knochendichte (Leitlinie Osteoporose 2009; mit freundlicher Genehmigung durch den DVO)

\begin{tabular}{c|c|c|c|c|c|c}
\hline \multirow{2}{*}{ Lebensalter in Jahren } & \multicolumn{5}{c}{$\begin{array}{c}\text { T-Wert } \\
\text { (Nur anwendbar auf DXA-Werte. Die Wirksamkeit einer medikamentösen Therapie ist bei T-Werten >-2,0 nicht belegt) }\end{array}$} \\
\hline Frau & Mann & $\mathbf{- 2 , 0}$ bis $\mathbf{- 2 , 5}$ & $\mathbf{- 2 , 5}$ bis -3,0 & $\mathbf{- 3 , 0}$ bis $\mathbf{- 3 , 5}$ & $\mathbf{- 3 , 5}$ bis -4,0 & $<-\mathbf{- 4 , 0}$ \\
\hline $50-60$ & $60-70$ & Nein & Nein & Nein & Nein \\
\hline $60-65$ & $70-75$ & Nein & Nein & Nein & Ja \\
\hline $65-70$ & $75-80$ & Nein & Nein & Ja & Ja \\
\hline $70-75$ & $80-85$ & Nein & Ja & Ja & Ja \\
\hline$>75$ & $>85$ & Ja & Ja & Ja & Ja \\
\hline
\end{tabular}


Die Therapiegrenzen verschob sich beim Vorliegen der in der Tabelle 6 gelisteten Risikofaktoren um 0,5 Einheiten nach oben. Ein 76-jähriger Mann, bei dem einer dieser Risikofaktoren vorlag, war demanch ab einem T-Score von -2,5 zu therapieren (und nicht ab einem T-Score von - 3,0). Lagen mehrere dieser Risikofaktoren vor, so addierten sie sich auf. Bei zwei oder mehr vorliegenden Risikofaktoren, konnte die Therapiegrenze maximal bis auf einen T-Score von -2 angehoben werden.

Tabelle 6: Risikofaktoren, die eine Anhebung der Therapiegrenze bedingen (Leitlinie Osteoporose 2009; mit freundlicher Genehmigung durch den DVO)

\begin{tabular}{|l|l|}
\hline \multicolumn{2}{|c|}{ Risikofaktoren, die eine Anhebung der Therapiegrenze bedingen } \\
\hline periphere Fraktur nach dem 50. Lebensjahr (B) & TSH $<0,3 \mathrm{mU} / \mathrm{I}$ (falls nicht behebbar) (B) \\
\hline singuläre Wirbelkörperfraktur 1. Grades (B) & Diabetes mellitus Typ 1 (B) \\
\hline proximale Femurfraktur eines Elternteils (B) & Rheumatoide Arthritis** \\
\hline multiple Stürze (B) & B II-Operation / Gastrektomie (B) \\
\hline Immobilität (B) & Epilepsie (B) \\
\hline Nikotinkonsum (B) & Hypogonadismus (B) (Serumtestosteron <200 ng/dl) \\
\hline subklinischer Hyperkortisolismus (C) & antiandrogene Therapie (B) \\
\hline primärer Hyperparathyreoidismus (konservativ behandelt (B) & Aromatasehemmertherapie** \\
\hline Wachstumshormonmangel bei Hypophyseninsuffizienz (B) & Deutlicher Knochendichteverlust ( $\geq 5 \%$ ) am Gesamtfemur über 2 Jahre (B) \\
\hline
\end{tabular}

Einen Sonderfall stellte die Therapie mit Glukokortikoiden dar. Wurden sie in einer Dosierung über 7,5 mg täglich für drei oder mehr Monate eingenommen, so bestand unabhängig vom Lebensalter eine dringende Empfehlung zur Initiierung einer antiosteoporosespezifischen Therapie, sofern der T-Score unter $-1,5 \mathrm{lag}$. Wurden sie einer Dosierung unter 7,5 mg täglich für drei Monate oder länger eingenommen, so hob sich die Therapiegrenze um eine Einheit an. Lagen noch weitere der gelisteten Risikofaktoren vor, so konnte die Therapiegrenze um bis zu zwei Einheiten angehoben werden. Davon ab konnte im Kontext des klinischen Gesamtbildes der Richtwert, ab dem eine Therapie empfohlen wurde, um bis zu einen T-Score abgesenkt werden. Patienten, die laut Abbildung 4 ein über 20\%iges 10-Jahres-Frakturrisiko hatten, bei denen sich aber basierend auf der DXA-Messung kein über 30\%iges 10-Jahres-Frakturrisiko ergab, wurden der Gruppe eines 20- bis 30\%igen 10-Jahres-Frakturrisikos zugeordnet.

\subsubsection{DVO-Leitlinien aus dem Jahr 2006}

Im Vergleich zur Fassung aus dem Jahr 2009 zeigten sich in den Leitlinien aus 2006 einige Abweichungen in Bezug auf die Risikofaktoren, ab denen eine Basisdiagnostik empfohlen wurde. Diese Faktoren sind in der Tabelle 7 gelistet. 
Tabelle 7: Anamnestische und klinische Risikofaktoren (Leitlinie Osteoporose 2006; mit freundlicher Genehmigung durch den DVO)

\begin{tabular}{|c|c|c|}
\hline Frau & Mann & Bei Vorliegen eines oder mehrerer der folgenden Befunde (sofern Risiko nicht behebbar): \\
\hline 50-60 Jahre & 60-70 Jahre & $\begin{array}{l}\text { - } \quad \text { eine oder mehrere Wirbelkörperfraktur(en) (A) } \\
\text { - } \quad \text { eine oder mehrere periphere Fraktur(en) als Einzelfallentscheidung (C) }\end{array}$ \\
\hline 60-70 Jahre & 70-80 Jahre & $\begin{array}{ll}\text { - } & \text { eine oder mehrere Wirbelkörperfraktur(en) (A) } \\
\text { - } & \text { eine oder mehrere periphere Fraktur(en) (A) } \\
\text { - } & \text { Schenkelhalsfraktur eines Elternteils (B) } \\
\text { - } & \text { Untergewicht (BMI < 20) (A) } \\
\text { - } & \text { Nikotinkonsum (A) } \\
\text { - } & \text { multiple Stürze (A) } \\
\text { Immobilität (A-B) }\end{array}$ \\
\hline$>70$ Jahre & $>80$ Jahre & Alle, falls daraus therapeutische Konsequenzen gezogen werden sollen/können (A) \\
\hline
\end{tabular}

Gesondert aufgeführt wurden Erkrankungen, die mit dem Vorliegen einer sekundären Osteoporose assoziiert sein können. Dazu zählen: Hypogonadismus, Hypercortisolismus, primärer Hyperparathyreodismus, systemische Glucocorticoide, höhergradige Niereninsuffizienz, Diabetes mellitus Typ I, Malassimilation, Antiepileptika und Anorexia nervosa. Für sie wurden keine geschlechts- oder altersspezifische Empfehlung ausgesprochen, ab wann weitere diagnostische Maßnahmen einzuleiten sind.

Analog zu den Leitlinien aus dem Jahr 2009 wurde eine Therapieempfehlung orientierend an den mittels DXA-Messung erhobenen T-Scores ausgesprochen. Die Richtwerte für Männer und Frauen waren dabei identisch zu den Leitlinien aus dem Jahr 2009. Unterschiede fanden sich in den Risikofaktoren, welche die Therapieuntergrenze anheben konnten. Diese Risikofaktoren umfassten: periphere Frakturen, Schenkelhalsfrakturen eines Elternteils, Nikotinkonsum, multiple Stürze und Immobilität. In Abhängigkeit vom Vorliegen dieser Risikofaktoren konnten die Therapiegrenze um bis zu einen T-Score angehoben werden.

\subsubsection{DVO-Leitlinien aus dem Jahr 2014}

Auf die Leitlinien aus dem Jahr 2014 wurde explizit im Punkt 1.3.1 eingegangen, sodass der Focus auf die Neuerungen im Vergleich zu 2009 liegt. Da die Leitlinie aus dem Jahre 2014 zum Zeitpunkt des Beginns der Datenerhebung noch nicht publiziert war, gibt es einige neu aufgeführte Risikofaktoren, die bei der Anamnese mancher Patienten nicht berücksichtigt wurden.

Die für die Studie relevanteste Änderung konzentrierte sich auf die Listung solcher Risikofaktoren, die ein über 20\%iges 10-Jahres-Frakturrisiko anzeigen. Hierbei handelte es sich v.a. um weitere Spezifizierungen der bereits in den Leitlinien von 2009 angeführten Risikofaktoren. Exemplarisch zu nennen ist der Parameter „nicht-vertebrale Frakturen“: So wurde er in den Leitlinien aus dem Jahr 2009 noch als „nicht-vertebrale Frakturen nach dem 50. Lebensjahr“ aufgeführt, wohingegen er in den Leilinien aus 2014 unter der erweiterten Definition „niedrigtraumatische nicht-vertebrale Frakturen mit Ausnahme von Finger-, Zehen-, Schädel- und 
Knöchelfrakturen“ angegeben wird. Diese Abwandlungen der in den älteren DVO-Leitlinien angegebenen Punkte, konnten problemlos aus den zur Verfügung stehenden Dokumenten entnommen werden. Bei weiteren Abänderungen der Leitlinien handelte es sich um neu angeführte Krankheitsbilder. Dazu zählten: Spondylitis ankylosans, Monoklonale Gammopathie unklarer Ursache, Herzinsuffizienz und Zöliakie. Sofern diese nicht explizit in den Arztbriefen vermerkt waren, galt die Annahme, dass diese Krankheitsbilder bei den Patienten nicht vorlagen. Darüber hinaus unterschieden sich die beiden Leitlinien, ab welchem Alter ein gelisteter Faktor weitere diagnostische Maßnahmen nach sich ziehen sollte. So wurde beispielsweise in den Leitlinien von 2009 bei Frauen ab dem 70. Lebensjahr eine weitere Diagnostik bei bestehender Therapie mit Aromatasehemmern empfohlen, in den Leitlinien aus dem Jahre 2014 bereits ab einem Alter von 60 Jahren.

Identisch waren für beide Leitlinien die empfohlenen Therapiegrenzen bezogen auf den TScore. Abweichungen zeigten sich bezüglich der Verschiebung der Therapiegrenzen. Im Gegensatz zu den Leitlinien aus dem Jahr 2009, wurden in der Fassung aus 2014 zusätzliche Faktoren gelistet, die die Therapiegrenze nicht nur um 0,5, sondern auch um 1,0 Einheiten anheben konnten. Dazu zählten neben den in den Leitlinien aus 2009 aufgeführten „Glukokortikosteroide oral $\geq 2,5 \mathrm{mg}$ und $<7,5 \mathrm{mg}$ Prednisolonäquivalent täglich (ausgenommen die Einnahme bei rheumathoider Arhtirits, in diesem Fall nur eine Anhebung der Therapiegrenze um 0,5 )" auch „Diabetes mellitus Typ I" sowie „drei und mehr niedrigtraumatische Frakturen in den letzten 10 Jahren als Einzelfallentscheidung (mit Ausnahme von Finder-, Zehen-, Schädelund Knöchelfrakturen)“. Auch kamen einige neue Faktoren hinzu, die die Therapiegrenze um 0,5 anzuheben vermochten. Diese sind in der Tabelle 8 gelistet. Insgesamt sollten nicht mehr als zwei Risikofaktoren addiert werden. Demzufolge war es möglich, die Therapiegrenze um bis zu zwei T-Scores anzuheben.

Tabelle 8: Neuerungen der Risikofaktoren, die die Therapiegrenze um +0,5 anheben (Leitlinie Osteoporose 2014)

\section{Anhebung der Therapiegrenzen um +0,5 - Neuerungen 2014}

\section{Herzinsuffizienz}

Protonenpumpeninhibitoren bei chronischer Einnahme

\section{Depressionen / Antidepressiva}

\section{Zöliakie}

Spondylitis ankylosans

Hyperthyreose oder subklinische Hyperthreose, sofern peristent

Glitazone

\section{HsCRP Erhöhung}

Knochenumbaumarker um 4. Quartil als Einzelfallerscheinung 


\subsubsection{Datenauswertung basierend auf dem internetbasierten FRAX-Rechner}

Basierend auf dem internetbasierten FRAX-Rechner ließ sich das 10-Jahresrisikos für eine HF, als auch MOF (klinische Wirbelfraktur, Unterarm, Hüft- oder Schulterfraktur) berechnen. Zu Beginn der Berechnung war die Länderzugehörigkeit auszuwählen. Da aus den Datenquellen keine eindeutigen Angaben zur Nationalität hervorgingen, wurde für die Auswertung die Eingabemaske für Deutschland verwendet. Der internetbasierte FRAX-Rechner integrierte 12 Risikofaktoren (Abbildung 5). Die Eingabe der Knochendichte war nicht zwingend erforderlich. Als Einheit für die Knochendichte wurde jeweils der niedrigste gemessene T-Score des Schenkelhalses verwendet.

In Bezug auf Alter und Gewicht wies der Algorithmus Limitationen auf. So akzeptierte das Modell nur Altersangaben zwischen 40 und 90 Jahren. Lagen die Altersangaben darunter, berechnet das Modell die Wahrscheinlichkeiten für 40-jährige Patienten, lagen die Angaben darüber, ging der Rechner von einem Alter von 90 Jahren für die Risikomodellierung aus. Auch das Gewicht musste für die Modellierung zwischen $25 \mathrm{~kg}$ und $125 \mathrm{~kg}$ liegen. Gewichtsangaben darüber oder darunter wurden Näherungen für das 10-Jahres-Risiko basierend auf den kleinstbzw. größtmöglichen Eingabeoptionen kalkuliert.

\section{Risikorechner}

Bitte beantworten Sie die untenstehenden Fragen für die Berechnung der 10-JahresWahrscheinlichkeit für eine Fraktur

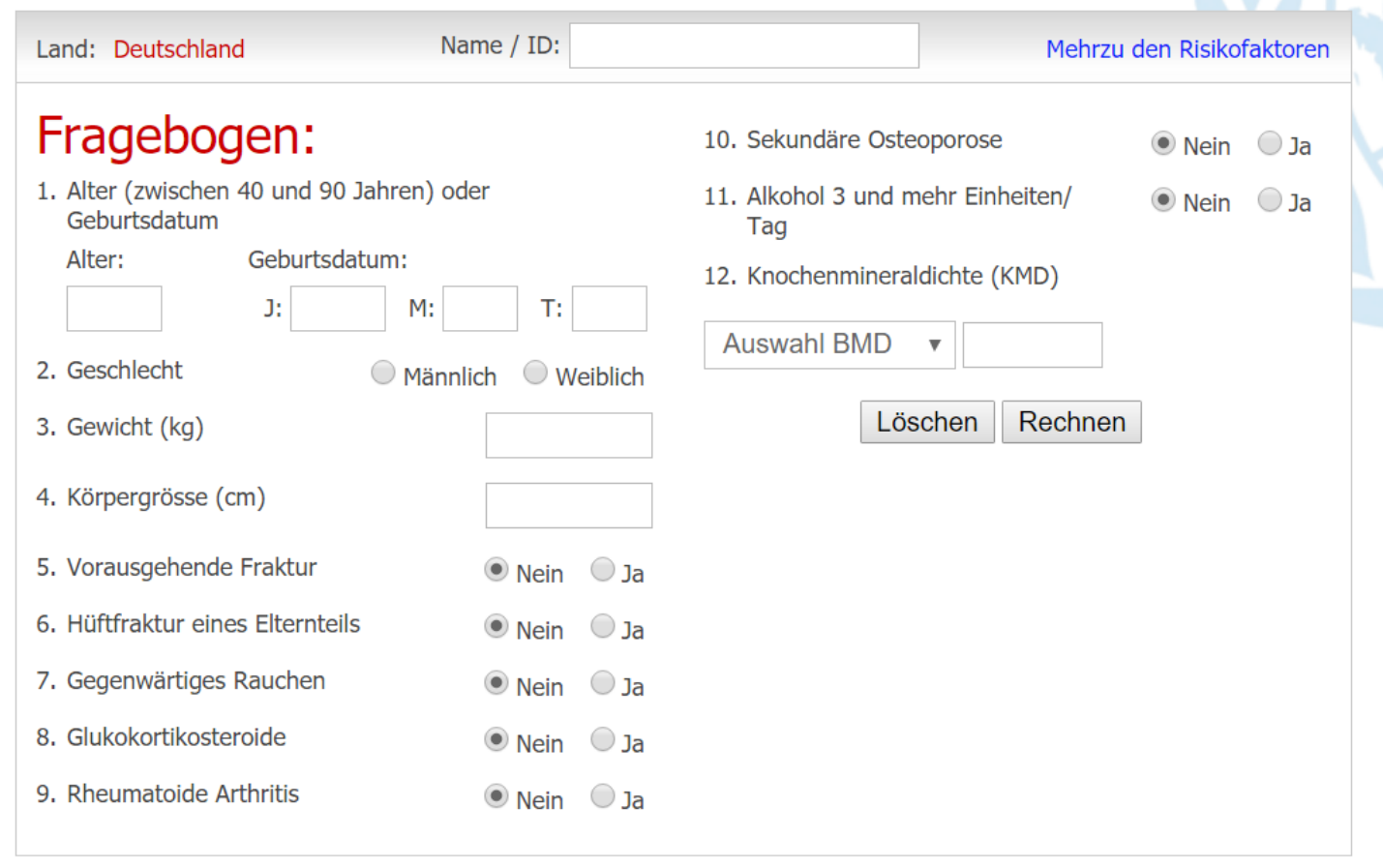


Einer detaillierteren Definition bedarf es bei den Risikofaktoren „vorausgehende Fraktur“, „Glukokortikosteroide“, „sekundäre Osteoporose“ und „Alkohol 3 und mehr Einheiten/Tag“. Die Auswahloption „Vorausgehende Fraktur" bezog sich nicht auf jegliche stattgehabte Fraktur, sondern nur auf Frakturen, die im Erwachsenenalter entweder spontan oder als Folge eines geringen Traumas, das bei gesunden Menschen nicht zu einer Fraktur geführt hätte, aufgetreten waren. Auch bei der Option "Glukokortikosteroide“ durfte nur die Antwortmöglichkeit ,ja“ gewählt werden, wenn eine derzeitige orale Therapie durchgeführt wurde, oder wenn zu einem früheren Zeitpunkt über einen Zeitraum von mindestens drei Monaten orale Glukokortikoide in einer täglichen oralen Dosis von mindestens $5 \mathrm{mg}$ eingenommen wurden. Gleiches galt für prednisolonäquivalente Dosierungen über $5 \mathrm{mg}$ pro Tag.

Unter dem Risikofaktor „sekundäre Osteoporose“ wurden für den FRAX-Rechner die folgenden Diagnosen verschlüsselt: Diabetes mellitus Typ I, Osteogenesis imperfecta bei Erwachsenen, langjährige, unbehandelte Hyperthyreose, Hypogonadismus, Eintritt der Menopause vor dem 45. Lebensjahr, chronische Mangelernährung oder Malabsorption und chronische Lebererkrankungen. Auch bei dem Risikofaktor „Alkohol 3 und mehr Einheiten/Tag“ bedarf es einer genaueren Erklärung in Bezug auf den Begriff „3 Einheiten“. Laut der offiziellen Seite der „University of Sheffield“ kann eine Einheit Alkohol zwischen $8 \mathrm{~g}$ und $10 \mathrm{~g}$ Alkohol enthalten, es zeigen sich leichte Variationen zwischen den einzelnen Ländern. Diese Menge entspricht etwa $285 \mathrm{ml}$, also einen Standardglas Bier, $30 \mathrm{ml}$ Spirituose, $60 \mathrm{ml}$ eines Aperitifs oder einem mittleren Glas Wein. In dem „Osteoporoserisiko-Fragebogen“, der im MVZ endokrinologikum Göttingen von den Patienten bei der Erstaufnahme ausgefüllt wurde, war eine Skala zur Erfassung der täglich konsumierten Menge alkoholischer Getränke abgebildet. Die Skala war in vier Schritte unterteilt: kein Alkohol, gelegentlich, bis 2/4 Wein und über 2/4 Wein. Als Äquivalent für die im FRAX erfragten „3 Einheiten Alkohol/Tag“ wurde der im „Osteoporoserisiko-Fragebogen“ angegebene Wert von „2/4 Wein pro Tag“ gewertet.

\subsubsection{1 „FRAX+“-Score}

Als Ergänzung wurde in dieser Arbeit eine Variation des FRAX-Rechners verwendet, der im Folgenden als „FRAX+“-Score bezeichnet wird. Der „FRAX+“-Score unterschied sich vom originalen FRAX-Score in der Auswahl der berücksichtigen Diagnosen unter dem Punkt „sekundäre Osteoporose“. Unter diesem Punkt wurden im „FRAX+“-Modell zusätzlich zu den im FRAX-Score berücksichtigen sekundären Osteoporoseformen die in der Tabelle 9 gelisteten Diagnosen berücksichtigt. Der „FRAX+“-Score war im Gegensatz zum FRAX-Score nicht validiert. 


\begin{tabular}{l}
\hline Zusätzliche sekundäre Osteoporoseform im „FRAX+“-Score \\
\hline Gastrointestinale Erkrankungen, Morbus Crohn \\
\hline Endokrinopathien \\
\hline Hyperkalzurie \\
\hline Antiepileptika \\
\hline Thyroxin-Einnahme \\
\hline Hypokalzurie \\
\hline Primärer Hyperparathyreodismus \\
\hline Genetik \\
\hline Zustand nach Transplantation \\
\hline Hyperthyreose \\
\hline Metastasen \\
\hline Vitamin D-Mangel \\
\hline Lokalisierte Osteoporose \\
\hline Heparin-/Marcumar-Einnahme \\
\hline Mastozytose
\end{tabular}

\subsubsection{Datenauswertung basierend auf dem 1-Jahres-Frakturrisiko nach C. Glüer}

Ausgehend von den Leitlinien aus dem Jahr 2014 wurde durch C. Glüer ein alternativer Evaluationsscore zur Abschätzung des Frakturrisikos entwickelt. Dieser orientiert sich am 1-Jahres-Frakturrisiko. Dieser Score wurde bisher nicht publiziert und wurde in dieser Arbeit mit der Einwilligung von Herrn Prof. Claus Glüer verwendet.

Das 1-Jahres-Frakturmodell basiert auf deutschen Daten zum Auftreten von Hüftfrakturen unter Berücksichtigung des internationalen Verhältnisses von radiographischen zu klinischen Wirbelkörperfrakturen. Auf einen Patienten mit einer klinischen Wirbelkörperfraktur kommen demnach 2,5 Patienten mit einer radiographischen Wirbelkörperfraktur (Fink et al. 2005). Da sich der Score nach C. Glüer auf das 1-Jahres-Frakturrisiko bezieht, erfolgt eine Ausklammerung der Mortalität. Das kleinstmögliche Risiko in diesem Modell lag in einem Bereich unter $0,3 \%$, das höchste über $10 \%$ in dem nächsten Jahr eine Fraktur zu erleiden. In dem Wertebereich dazwischen erfolgte die Angabe des Frakturrisikos als absoluter Prozentwert und nicht als Wertebereich. Um das Patientenkollektiv detaillierter beschreiben zu können, wurden die zwei Patientengruppen, die ein unter 0,3\%iges bzw. über 10\%iges Frakturrisiko hatten, umcodiert. Jedem Patienten mit einem unter 0,3\%igem Risiko wurde ein Frakturrisiko von 0,29\% zugeordnet, jedem Patienten mit einem über 10\%igem Jahres-Frakturrisiko ein 10,01\%iges. Dabei konnte nicht berücksichtig werden, dass es beispielsweise Patienten deren Risiko unter $0,3 \%$ lag auch einen Wert von $0,1 \%$ annehmen konnten. 
Die Kalkulation orientierte sich an Risikofaktoren, die in den DVO-Leitlinien aus dem Jahr 2014 gelistet waren. Diese wurden in Abhängigkeit von ihrer prognostischen Relevanz für das Auftreten von osteoporoseassoziierten Frakturen in drei Gruppen unterteilt: moderate, starke und sehr starke Risikofaktoren (Tabelle 10).

Tabelle 10: Risikofaktoren für die Kalkulation des 1-Jahres-Frakturrisiko nach C. Glüer (gruppiert nach prognostischer Relevanz)

\begin{tabular}{|c|c|c|}
\hline Moderate Risikofaktoren & Starke Risikofaktoren & Sehr starke Risikofaktoren \\
\hline $\begin{array}{l}\text { Singuläre Wirbelkörperfraktu- } \\
\text { ren 1. Grades mit Deckplatten- } \\
\text { impression }\end{array}$ & $\begin{array}{l}\text { Zwei nicht-vertebrale Frakturen } \\
\text { nach dem 50. Lebensjahr }\end{array}$ & $\begin{array}{l}\text { Bestehende oder geplante } \\
\text { Therapie mit oralen Glukokor- } \\
\text { tikoiden } \geq 7,5 \text { mg prednisolo- } \\
\text { näquivalent täglich für }>3 \text { Mo- } \\
\text { nate im Jahr }\end{array}$ \\
\hline $\begin{array}{l}\text { Singuläre nichtvertebrale Frak- } \\
\text { turen nach dem 50. Lebensjahr }\end{array}$ & $\begin{array}{l}\text { Cushing Syndrom oder subkli- } \\
\text { nischer Hypercortisolismus }\end{array}$ & $\begin{array}{l}\text { Niedrigtraumatische Wirbelkör- } \\
\text { perfraktur singulär } \geq 2 \text {. Grades, } \\
\text { multiple } \geq 1 \text {. Grades, sofern } \\
\text { eine andere Ursache der Frak- } \\
\text { tur nicht wahrscheinlich ist }\end{array}$ \\
\hline $\begin{array}{l}\text { Proximale Femurfraktur bei Va- } \\
\text { ter oder Mutter }\end{array}$ & $\begin{array}{l}\text { Primärer Hyperparathyreodis- } \\
\text { mus }\end{array}$ & $\begin{array}{l}\text { Niedrigtraumatische multiple } \\
\text { periphere Frakturen mit Aus- } \\
\text { nahme von Knöchel-, Handy-, } \\
\text { Finger und Gesichtsfrakturen }\end{array}$ \\
\hline Immobilität & Wachstumshormonmangel & \\
\hline Untergewicht & $\begin{array}{l}\text { Subklinische oder manifeste } \\
\text { Hyperthyreose }\end{array}$ & \\
\hline hs CRP erhöht & $\begin{array}{l}\text { Diabetes mellitus Typ } 1<70 \\
\text { Jahre }\end{array}$ & \\
\hline Hyponatriämie & B-II-Resektion, Gastrektomie & \\
\hline Rheumatoide Arthritis & Herzinsuffizienz & \\
\hline Spondylitis ankylosans & Antiepileptika & \\
\hline Zöliakie & Glitazone & \\
\hline \multicolumn{3}{|l|}{ Rauchen und/oder COPD } \\
\hline \multicolumn{3}{|l|}{ Antiandrogene Therapie } \\
\hline \multicolumn{3}{|l|}{ Aromatasehemmer } \\
\hline \multicolumn{3}{|l|}{ Glukokortikosteroide oral } \\
\hline \multicolumn{3}{|l|}{ Glukokortikosteroide inhalativ } \\
\hline \multicolumn{3}{|l|}{$\begin{array}{l}\text { Sturzbegünstigende Medika- } \\
\text { mente }\end{array}$} \\
\hline \multicolumn{3}{|l|}{ Protonenpumpeninhibitoren } \\
\hline \multicolumn{3}{|l|}{ Dopaminerge Medikamente } \\
\hline Schleifendiuretika & & \\
\hline
\end{tabular}

Jedem dieser Risikogruppen wurde ein relatives Risiko zugeordnet. Für moderate Risikofaktoren betrug es den Faktor 1,5, für starke den Faktor 3 und für sehr starke Risikofaktoren den Faktor 6. Als Grundannahme wurde vorausgesetzt, dass zwischen den einzelnen Faktoren keine Interaktionen bestand. Das 1-Jahres-Frakturrisiko ergab sich aus der Kombination 
der individuellen Risikofaktoren unter Berücksichtigung des Alters und des Geschlechts. Als Grundlage für die Risikoevaluation im vorliegenden Kollektiv männlicher Patienten diente die Abbildung 6.

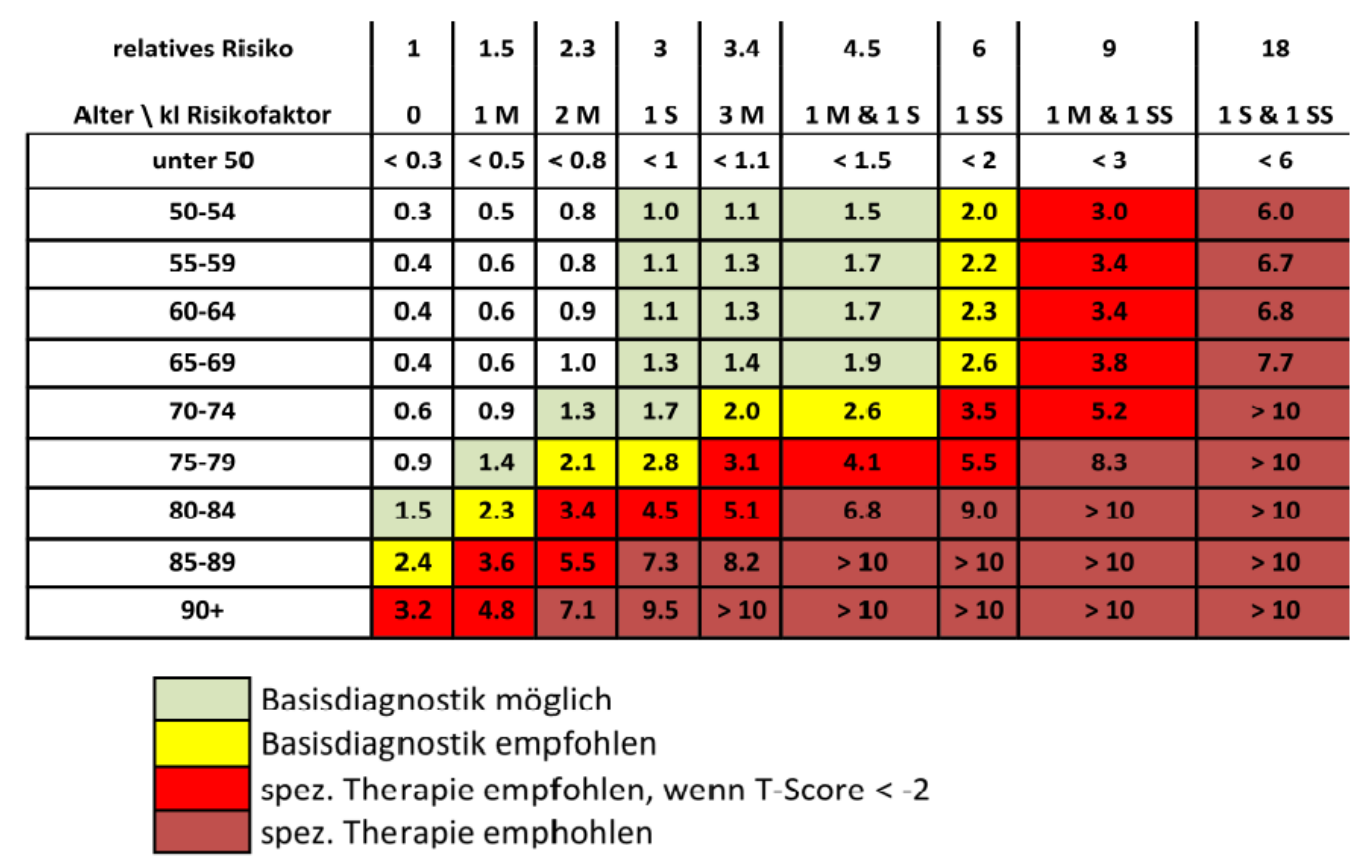

Abbildung 6: 1-Jahres-Frakturrisiko für Hüft- und radiologische Wirbelkörperfrakturen in Abhängigkeit von Alter und Anzahl bzw. Art der Risikofaktoren (Männer) (M=moderat, S= stark, SS= sehr stark) (Gluer 2013; mit freundlicher Genehmigung durch C. Glüer)

Lagen beispielsweise bei einem 56-jährigen männlichen Patienten drei moderate Risikofaktoren vor, so ergab sich ein 1,3\%iges 1-Jahres-Frakturrisiko. Das relative Risiko errechnete sich durch die Multiplikation der einzelnen relativen Risiken. Bei drei moderaten Risikofaktoren ergab sich so ein relatives Risiko von gerundet $3,4(1,5 \times 1,5 \times 1,5=3,375)$. Traten Konstellationen an Risikofaktoren auf, die nicht in der Tabelle abgebildet waren, so wurde in der folgenden Auswertung das höchste mögliche Risiko verwendet. Ergaben sich für einen 56-jähriger Patienten beispielsweise zwei moderate und zwei starke Risikofaktoren, so wurde das 1-JahresFrakturrisiko für einen moderaten und einen starken Risikofaktor verwendet (Frakturwahrscheinlichkeit 1,75\%; relatives Risiko 4,5).Zusätzlich ließen sich der Abbildung 6 diagnostische und therapeutische Empfehlungen entnehmen. Ab einem 1\%igen 1-Jahres-Frakturrisiko konnte eine Basisdiagnostik durchgeführt werden, und ab einem $2 \%$ igem Risiko sollte sie durchgeführt werden. Die Indikation für eine spezifische antiosteoporotische Therapie bestand $a b$ einem über $3 \%$ igem Bruchrisiko und einem gemessenen T-Score unter $-2,0$. Ab einem 1Jahres-Frakturrisiko über $6 \%$ sollte unabhängig vom ermittelten T-Score mit einer osteoporosespezifischen Therapie begonnen werden.

Die Abbildung 7 gibt die möglichen Kombinationen an Risikofaktoren mit den zugehörigen Therapieschwellen wieder. Auch hier galt, dass beim Auftreten einer Kombination an 
Risikofaktoren, die nicht in der Tabelle aufgeführt wurde, die höchst mögliche Kombination an Risikofaktoren angenommen wurde. Lagen so beispielsweise bei einem 56-jähriger Mann zwei moderate und zwei schwere Risikofaktoren vor, so bezog sich die Therapiegrenze auf einen moderaten und einen schweren Risikofaktor. Eine Therapie sollte folglich initiiert werden, sofern ein T-Score von - 2,0 oder niedriger gemessen wurde. Bei allen grau hinterlegten Therapieindikationsschwellen ergab sich durch die vorliegenden Kombinationen an Risikofaktoren ein mindestens 3\%iges 1-Jahres-Frakturrisiko. Demnach musste der gemessene T-Score nicht unter - 2,0 liegen, damit eine spezifische Therapie indiziert war.

\begin{tabular}{|c|c|c|c|c|c|c|c|c|c} 
Alter \ kI Risikofaktor & 0 & $1 \mathrm{M}$ & $2 \mathrm{M}$ & $1 \mathrm{~S}$ & $3 \mathrm{M}$ & $1 \mathrm{M} \& 1 \mathrm{~S}$ & $1 \mathrm{SS}$ & $1 \mathrm{M} \& 1 \mathrm{SS}$ & $1 \mathrm{~S} \& 1 \mathrm{SS}$ \\
\hline unter 50 & $<-3.9$ & $<-3.3$ & $<-2.8$ & $<-2.3$ & $<-2.2$ & $<-1.7$ & $<-1.3$ & $<-0.7$ & $<0.3$ \\
\hline $50-54$ & -3.9 & -3.3 & -2.8 & -2.3 & -2.2 & -1.7 & -1.3 & -1.7 & -0.7 \\
\hline $55-59$ & -3.8 & -3.2 & -2.6 & -2.2 & -2.0 & -1.6 & -1.2 & -1.6 & -0.6 \\
\hline $60-64$ & -3.8 & -3.2 & -2.6 & -2.2 & -2.1 & -1.6 & -1.2 & -1.6 & -0.7 \\
\hline $65-69$ & -3.7 & -3.1 & -2.5 & -2.1 & -2.0 & -1.6 & -1.2 & -1.6 & -0.6 \\
\hline $70-74$ & -3.3 & -2.8 & -2.2 & -1.9 & -1.7 & -1.3 & -1.9 & -1.3 & -0.4 \\
\hline $75-79$ & -2.9 & -2.3 & -1.8 & -1.4 & -1.3 & -1.8 & -1.4 & -0.9 & 0.0 \\
\hline $80-84$ & -2.5 & -1.9 & -1.4 & -1.9 & -1.8 & -1.4 & -1.0 & -0.5 & 0.4 \\
\hline $85-89$ & -2.2 & -1.6 & -1.1 & -1.6 & -1.5 & -1.1 & -0.8 & -0.3 & 0.6 \\
\hline 90 & -2.0 & -1.5 & -1.9 & -1.5 & -1.4 & -1.0 & -0.7 & -0.2 & 0.7 \\
\hline
\end{tabular}

T-Score $<-2$ nötig

T-Score $<-2$ nicht nötig

Abbildung 7 Therapieindikationsschwellen für Männer in Abhängigkeit vom Alter, Risikofaktoren und T-Score $(M=$ moderat, $S=$ stark, SS= sehr stark; blau: Therapieempfehlung in Abhängigkeit vom angegebenen T Score; gelb: Therapieempfehlung ab einem T-Score <-2; grau: Therapieempfehlung auch bei einem T-Score >-2) (Gluer 2013; mit freundlicher Genehmigung durch C. Glüer)

\subsection{Statistische Auswertung}

Die statistische Auswertung erfolgten nach Rücksprache mit wissenschaftlichen Mitarbeitern des Instituts für Medizinische Statistik der Georg-August-Universität Göttingen unter Verwendung von SPSS und STATA.

Mittels explorativer Datenanalyse in SPSS wurde die einzelnen Patientencharakteristika sowie die Frakturrisiko-Scores (DVO-, FRAX-, „FRAX+“-Score, Score nach C. Glüer) ausgewertet. Die Testung auf Normalverteilung erfolgte nach Kolmogorow-Smirnow (Signifikanzniveau: $p=0,05)$.

Der Wilcoxon-Rang-Vorzeichen-Test für abhängige, nichtparametrische Stichproben wurde zum Vergleich der zentralen Aussagetendenzen der verschiedenen Scores verwendet, sofern diese mindestens ordinalskaliert waren (Methodenberatung der Universität Zürich). Der McNemar-Test wurde verwendet, sofern es um den Vergleich dichotomer Merkmale ging. Er zählt zur Gruppe der Chi-Quadrat-Tests. 
Zusätzlich wurde die Rangkorrelation nach Kendall-tau-b durchgeführt. Diese war besonders bei kleinen Stichprobenumfängen robuster gegenüber Ausreißern. Durch sie war es möglich, den ungerichteten linearen Zusammenhang zweier intervallskalierter Variablen zu berechnen. Dabei galt: je näher der Korrelationskoeffizient an dem Betrag von 1 war, desto stärker war der lineare Zusammenhang der untersuchten Variablen. Der Korrelationskoeffizient konnte sowohl positiv als auch negativ sein. Ein positiver Korrelationskoeffizient entsprach einem gleichsinnigen Zusammenhang, wohingegen ein negativer einem gegensinnigen Zusammenhang entsprach. Diese Analyse wurde für den Vergleich der einzelnen, intervallskalierten FRAXScores untereinander, sowie mit dem Score nach C. Glüer angewandt.

Als Maß der Interrater-Reliabilität zwischen zwei Frakturrisikoscores in Bezug auf die Therapieentscheidungen wurde Cohens Kappa $(K)$ verwendet. Durch dieses statistische Verfahren ließ sich prüfen, ob das Ausmaß an Übereinstimmung zwischen zwei untersuchten Scores höher war, als das Ausmaß an zufälligen Übereinstimmungen. Ein Kappa ( $\mathrm{k}$ ) von 0,81 - 1,0 galt dabei als (fast) vollkommene Übereinstimmung, eine $\mathrm{k}$ von $0,61-0,8$ als beachtliche Übereinstimmung, eine $k$ von 0,41 - 0,6 als mittelmäßige Übereinstimmung, ein $k$ von 0,21 0,4 als ausreichende Übereinstimmung, ein $\mathrm{k}$ von $0-0,2$ als etwas Übereinstimmung und ein $\mathrm{K}<0$ als schlechte Übereinstimmung.

Abschließend wurde für die einzelnen FRAX- Scores eine multiple lineare Regressionsanalyse durchgeführt. Ziel der Regressionsanalyse war es, eine abhängige Variable (y) durch eine oder mehrere unabhängige Variablen $(x)$ zu erklären bzw. zu prognostizieren. Dabei war es möglich, sowohl die Stärke des Einflusses,jeder einzelnen unabhängigen Variablen auf die abhängige Variable zu bestimmen als auch die Veränderung der abhängigen Variablen bei einer Variation der unabhängigen Variablen. Diese Aussagen ließen sich aus dem Regressionskoeffizienten $(\beta)$ ablesen: Stieg die unabhängige Variable um eine Einheit an, so stieg die abhängige Variable um den durch den Regressionskoeffizienten angegebenen Wert. Bei einem negativen Regressionskoeffizienten fiel die abhängige Variable um den durch den Koeffizienten angegeben Wert. Das entsprechende statistische Modell stellt sich wie folgt dar:

$y=\beta_{0}+\beta_{1} \cdot X_{1}+\beta_{2} \cdot X_{2}+\ldots+\beta_{k} \cdot X_{k}+\varepsilon_{i}$

$y=$ Schätzer der abhängigen Variabel

$\mathrm{xk}=$ unabhängige Variable $\mathrm{k}$

$\beta k=$ Regressionskoeffizient der Variable $x k$

$\varepsilon \mathrm{i}=$ Fehlerterm des Probanden $\mathrm{i}$

Der Anteil der erklärten Varianz an der Gesamtvarianz der abhängigen Variablen ließ sich durch den Determinationskoeffizienten $\mathrm{R}^{2}$ beschreiben. Je näher er an dem Wert eins lag, desto genauer war das Regressionsmodell. Auch hier zeigte sich, dass durch den Einschluss von vielen unabhängigen Variablen der Koeffizient zwar höher lag, aber die Genauigkeit abnahm. Um diesen Fehler zu umgehen wurde im Folgenden der korrigierte 
Determinationskoeffizient verwendet. Er berücksichtigte nur solche unabhängigen Variablen, die einen erklärenden Anteil an der Zielvariablen hatten.

Bei der abhängigen Variablen handelte es sich in den Analysen um die mittels der FRAXEingabemaske errechneten 10-Jahres-Frakturrisiko-Prognosen. Als unabhängige Variablen flossen alle in den FRAX-Rechner eingegebenen Parameter ein. Bis auf Alter und BMI handelte es sich dabei um binäre Variablen. Diese wurden in Dummy-Variablen umcodiert, wobei „0“ gleichzusetzen war mit der Antwortoption „nein“ und „1“ mit der Antwortoption „ja“.

Die Testung auf Multikollinearität erlaubte eine Aussage, ob zwischen zwei oder mehreren der unabhängigen Variablen im untersuchten Modell eine starke Korrelation bestand. Dies hätte zur Folge gehabt, dass die Schätzung der Regressionskoeffizienten in dem untersuchten Modell zunehmend ungenauer wird. Um das Maß der Multikollinearität einzuschätzen, wurde der Varianzinflationsfaktor verwendet. Sofern dieser $>10$ war, konnte von einer Multikollinearität gesprochen werden (Backhaus et al. 2013). 


\section{Ergebnisse}

\subsection{Patientenkollektiv}

Insgesamt standen für 147 männliche Patienten, die im Zeitraum von 01.07.2007 - 30.06.2014 im medizinischen Versorgungszentrum MVZ endokrinologikum Göttingen zur Erstdiagnostizierung einer Osteoporose vorstellig waren, ein vollständiger Datensatz an Erstaufnahmedokumenten und eine schriftliche Einwilligung zur Verwendung der Daten zur Verfügung, sodass sie in die Studie aufgenommen werden konnten. Lediglich bei 17 Patienten wurde die DXAMessung am Schenkelhals im Rahmen der Erstvorstellung nicht durchgeführt bzw. nicht zeitnah nachgeholt. Trotzdem wurden diese Patienten in die Studie aufgenommen. An gegebener Stelle wurden diese auf Grund der fehlenden Daten aus der Auswertung ausgeschlossen.

\subsection{Deskriptive Statistik}

\subsubsection{Alter}

Im Schnitt lag das Patientenalter bei 58,9 Jahren, der jüngste Patient war 41 Jahre, der älteste 89 Jahre. Die Standardabweichung vom Mittelwert betrug 10,6 Jahre. Bei Überprüfung des Alters auf eine Normalverteilung zeigte sich, dass der Parameter unter Annahme eines Signifikanzniveaus von $5 \%$ nicht normalverteilt war. Die Abbildung 8 stellt die Altersverteilung als Normalverteilungsdiagramm dar. 50\% aller Patienten lagen bezüglich der Altersverteilung zwischen 51 und 67 Jahren.

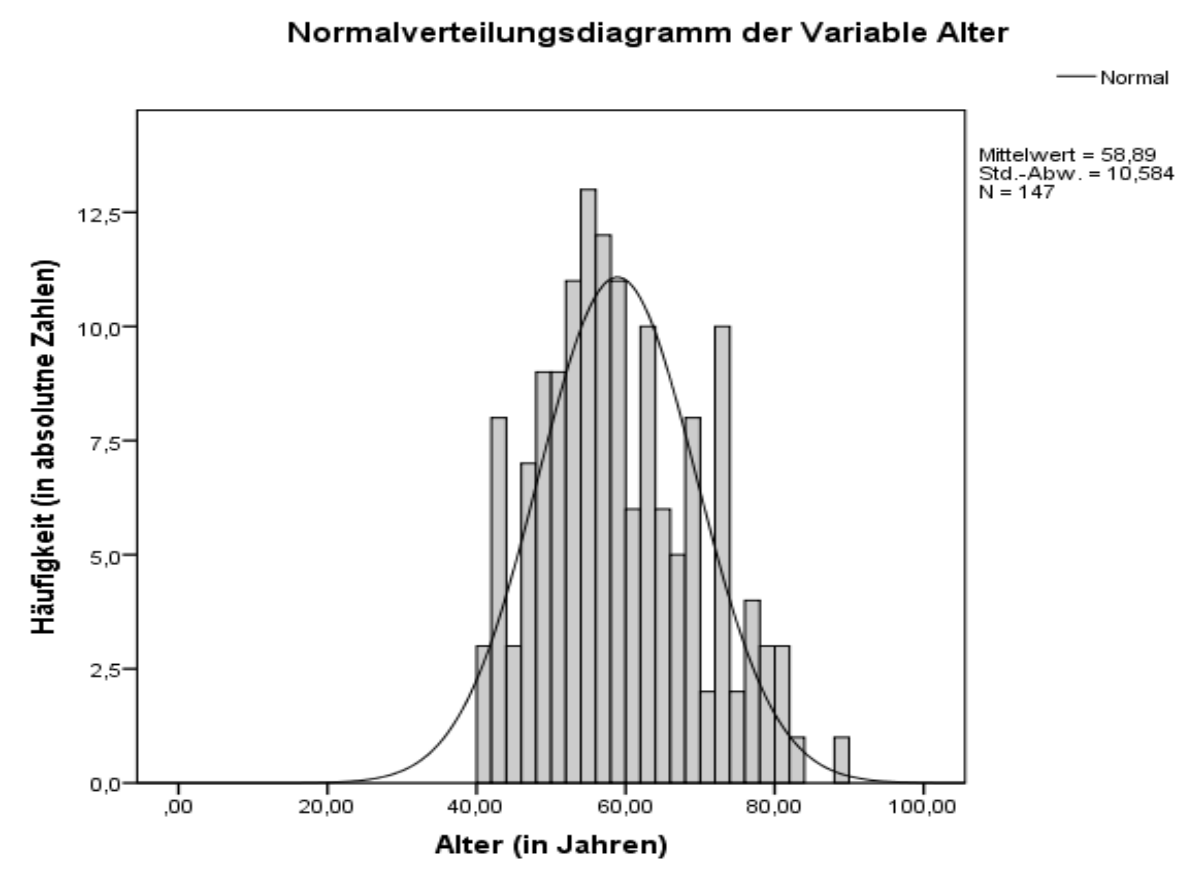

Abbildung 8: Normalverteilungsdiagramm der Variable Alter 


\subsubsection{Körpergröße, Gewicht, Body Mass Index (BMI)}

Bei den Parametern Gewicht und Body-Mass-Index handelte es sich auf dem Signifikanzniveau von $5 \%$ um normalverteilte Daten, die Körpergröße war dahingegen nicht normalverteilt.

Das Gewicht der männlichen Patienten des Studienkollektivs lag zwischen 47,6 kg und 121,3 $\mathrm{kg}$. Es zeigte sich ein mittleres Gewicht von $82,82 \mathrm{~kg}$ mit einer Standardabweichung von \pm 15,07 kg. Die Patienten maßen im Schnitt eine Körpergröße von 175,9 cm mit einer Standardabweichung von $\pm 8,1 \mathrm{~cm}$. Der kleinste Patient war $158,3 \mathrm{~cm}$, der größte 212,0 cm groß. Die Boxplots in der Abbildung 9 veranschaulichen die Verteilung von Gewicht und Größe im Studienkollektiv. 50\% der Patienten wogen zwischen 72,25 und 92,95 kg bzw. waren zwischen 169,85 und $181 \mathrm{~cm}$ groß.
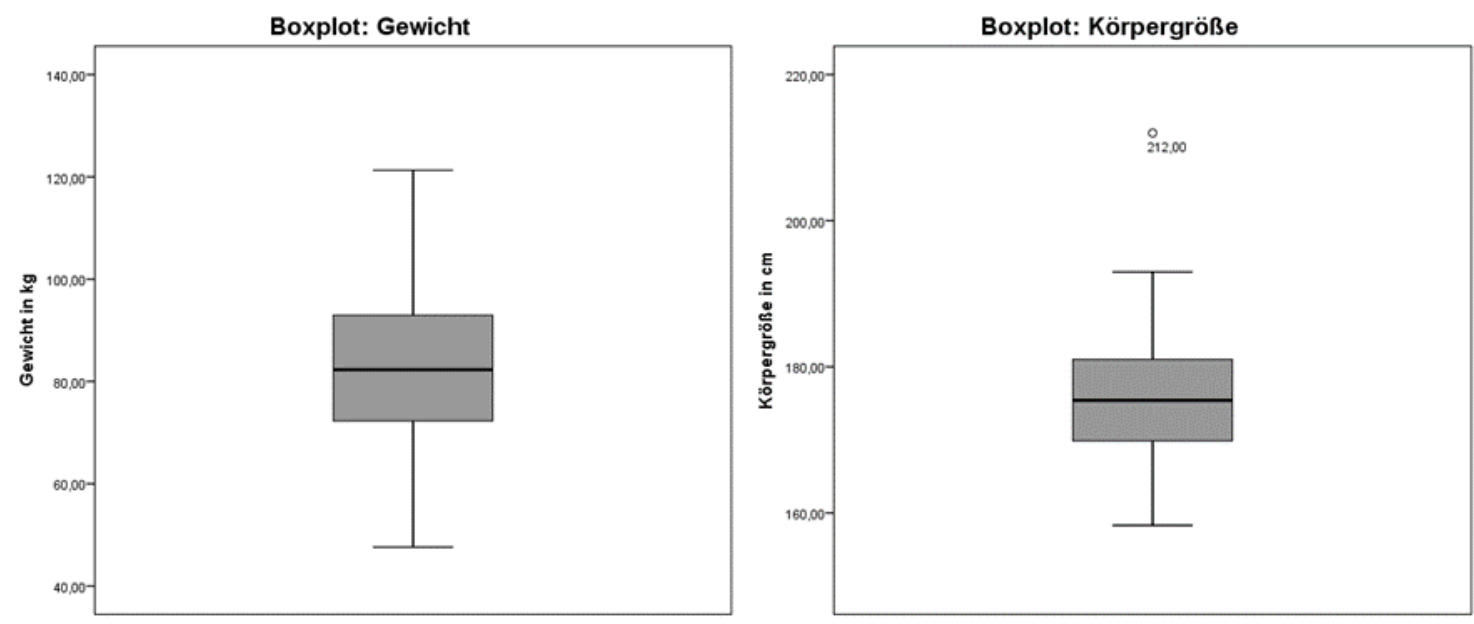

Abbildung 9: Boxplots: Gewicht (in kg) und Körpergröße (in cm) ( $n=147$ )

Aus Gewicht und Körpergröße ließ sich anhand der folgenden Formel der Body Mass Index (BMI) berechnen: BMI $\left(\mathrm{kg} / \mathrm{m}^{2}\right)=\frac{\mathrm{kg}}{(\mathrm{m})^{2}}(K=$ Körpergewicht in Kilogramm, $G=$ Körpergewicht in Meter)

Im Studienkollektiv errechnete sich ein durchschnittlicher BMI von 26,67 (Übergewicht im Stadium der Präadipositas) mit einer Standardabweichung von $\pm 4,652$. Anhand der errechneten BMI-Werte ließ sich eine Klassifizierung in die Körpergewichtsklassen vornehmen (Abbildung 10). Es zeigte sich, dass $63,27 \%$ (93 von 147) der männlichen Patienten des Studienkollektivs übergewichtig waren. Dieser Wert liegt über dem durchschnittlichen Anteil der übergewichtigen Erwachsenen in der deutschen Normalbevölkerung. Hier finden sich ca. 50\% mit einem BMI-Wert $\geq 25$ (Allolio und Schulte 2010). Sechs der 147 (4,08\%) männlichen Patienten wiesen einen BMI unter 18,5 auf und waren somit untergewichtig. 


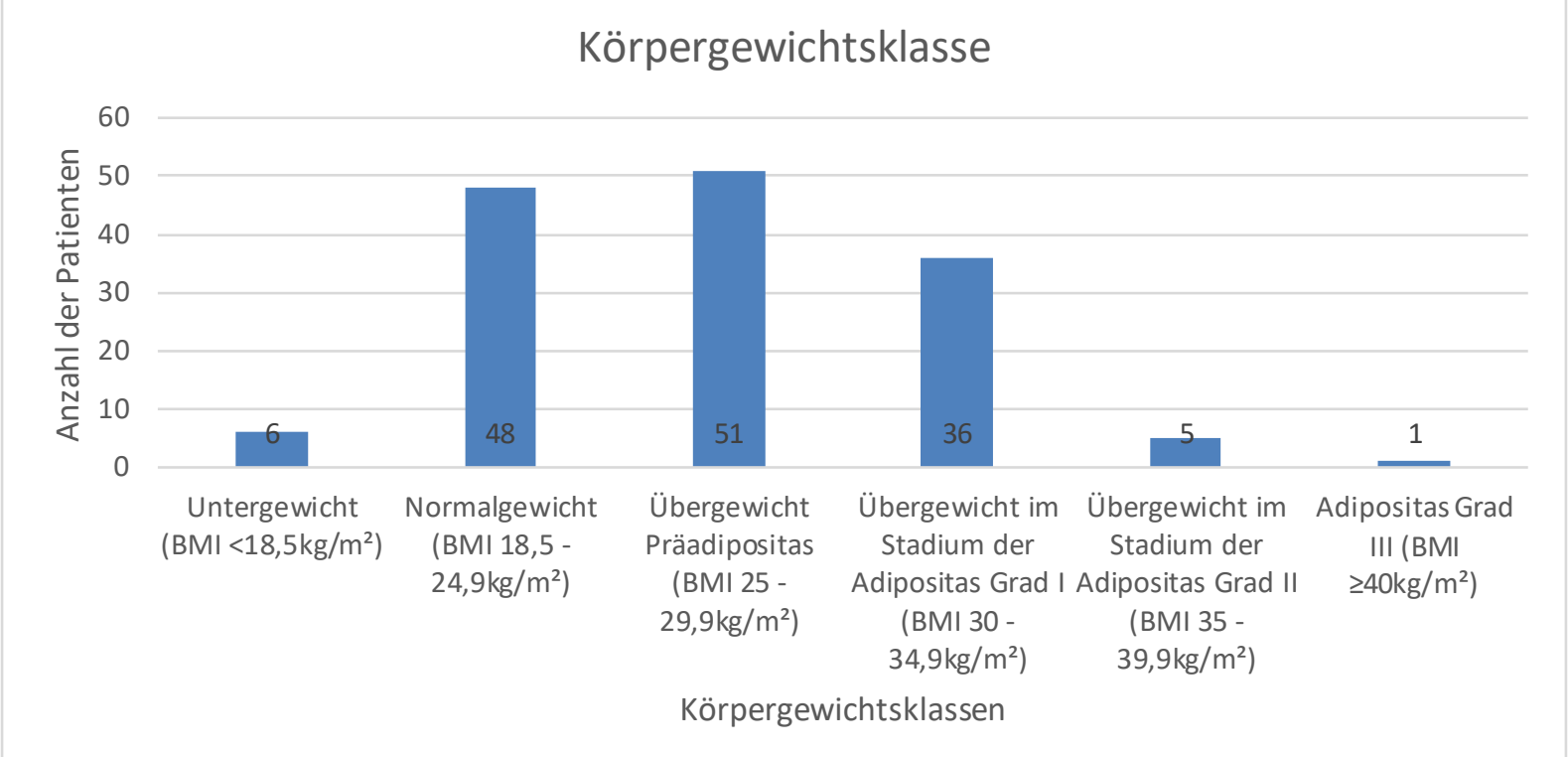

Abbildung 10: Anzahl der Patienten pro Körpergewichtsklasse

\subsubsection{Frakturen}

Die statistische Auswertung der Frakturen erfolgte gruppiert nach Frakturtypen, die im DVObzw. FRAX-Score als Risikofaktoren gelistet sind. Es erfolgte eine Aufschlüsselung nach den folgenden Frakturtypen:

- $\quad$ singuläre oder multiple niedrigtraumatische Wirbelkörperfrakturen 1.-3. Grades

- niedrigtraumatische singuläre Wirbelkörperfrakturen > 2. Grades oder multiple Wirbelkörperfrakturen > 1. Grades

- niedrigtraumatische multiple periphere Frakturen mit Ausnahme von Knöchel-, Hand-, Finger- und Gesichtsfrakturen

- „FRAX-relevante“ Frakturen.

Abbildung 11 zeigt die vier verschiedenen Frakturkategorien im Hinblick auf die Optionen „Fraktur ja“ oder „Fraktur nein“. In jeder Kategorie wurden insgesamt 147 Patienten berücksichtigt. Die höchste Anzahl an Frakturaufkommen lag in der Kategorie „FRAX-relevante Frakturen“ vor (dazu zählen alle im Erwachsenenalter spontan oder als Folge eines (leichten) Traumas aufgetretenen Frakturen, die bei einem gesunden Menschen nicht zu einer Fraktur geführt hätten). Hier wiesen 85 von 147 (57,8\%) Patienten ein Frakturereignis auf. Die wenigsten stattgehabten Frakturen waren in der Kategorie "niedrigtraumatische multiple periphere Frakturen mit Ausnahme von Knöchel-, Hand-, Finger- und Gesichtsfrakturen“ zu verzeichnen. Bei nur 11 von 147 Patienten (7,5\%) war ein solcher Frakturtyp dokumentiert. „Singuläre oder multiple niedrigtraumatische Wirbelkörperfrakturen 1.-3. Grades“ waren bei 68 von 147 (46,3\%), „niedrigtraumatische singuläre Wirbelkörperfrakturen > 2.Grades oder multiple Wirbelkörperfrakturen > 1. Grades“ bei 44 von 147 (29,9\%) Patienten dokumentiert. 


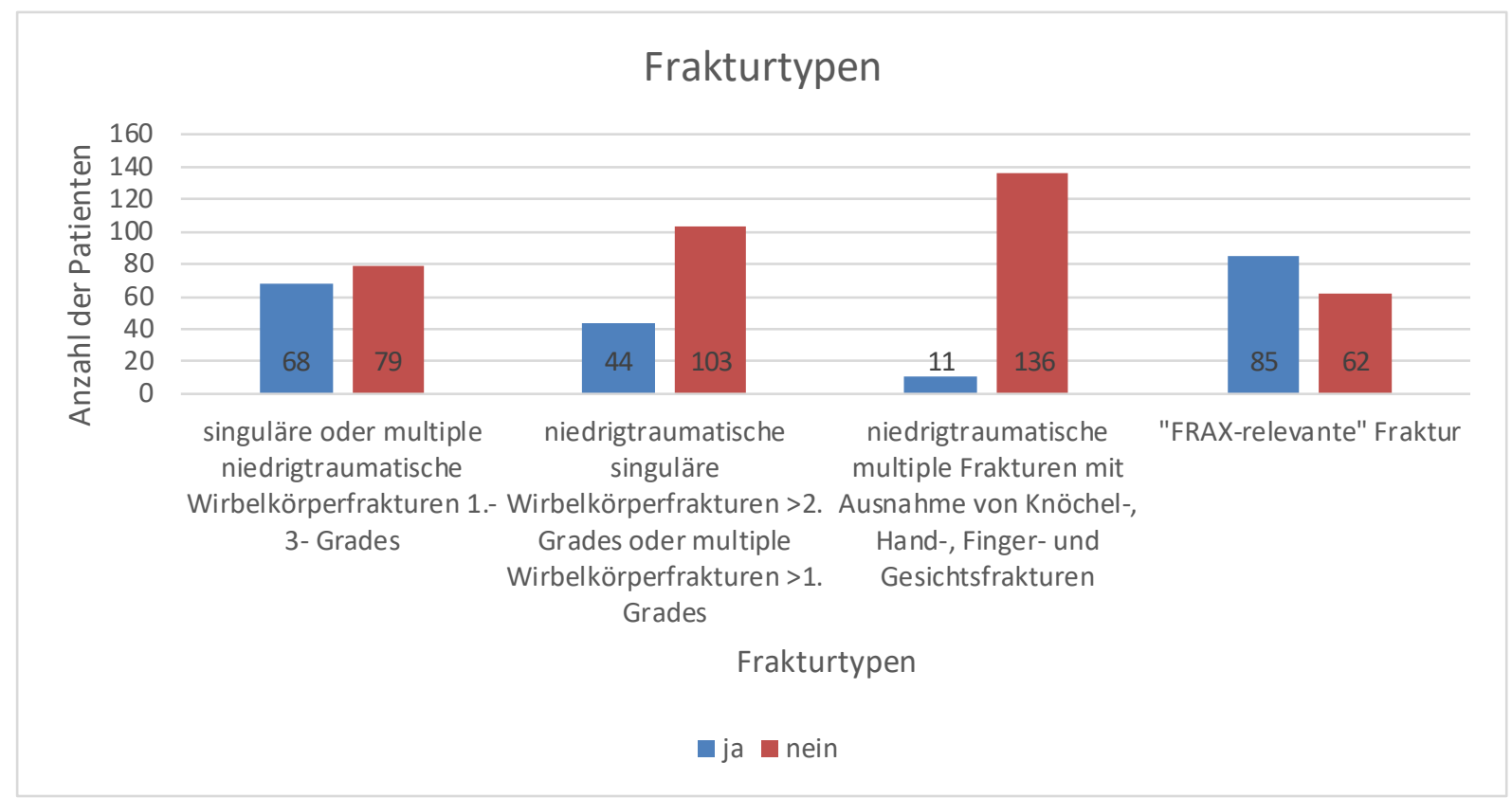

Abbildung 11: Frakturtypen

\subsubsection{Femurfraktur bei einem der beiden Elternteile}

Das Vorliegen von Femurfrakturen bezog sich auf anamnestische Patientenangaben. Darauf basierend war keine genauere Aufschlüsselung möglich, ob eine Femurfraktur bei nur einem oder beiden Elternteilen aufgetreten war. Sofern die Patientenunterlagen keine genauen Angaben über dieses Ereignis ergaben, wurde dies als nicht stattgehabtes Ereignis gewertet.

Bei 14 der 147 (9,5\%) männlichen Patienten lag eine Fraktur des Femurs bei einem der beiden Elternteile vor. Bei den übrigen 133 (90,5\%) Patienten konnte entweder mit Sicherheit gesagt werden, dass bei keinem der Elternteile eine Femurfraktur bis zum Zeitpunkt aufgetreten war, oder es konnte keine sicheren Angaben diesbezüglich gemacht werden.

Femurfraktur bei einem der beiden Elternteile

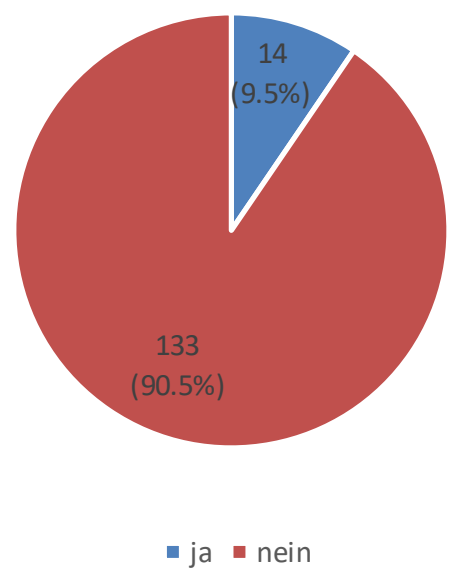




\subsubsection{Gegenwärtiges Rauchen}

Anhand des osteoporosespezifischen Fragebogens des MVZ endokrinologikum Göttingen war eine Einordnung des gegenwärtigen Zigarettenkonsums in die folgenden vier Gruppen möglich: „keine Zigaretten“, „unter 10 Zigaretten pro Tag“, „10 - 20 Zigaretten pro Tag“ und „über 20 Zigaretten pro Tag“.

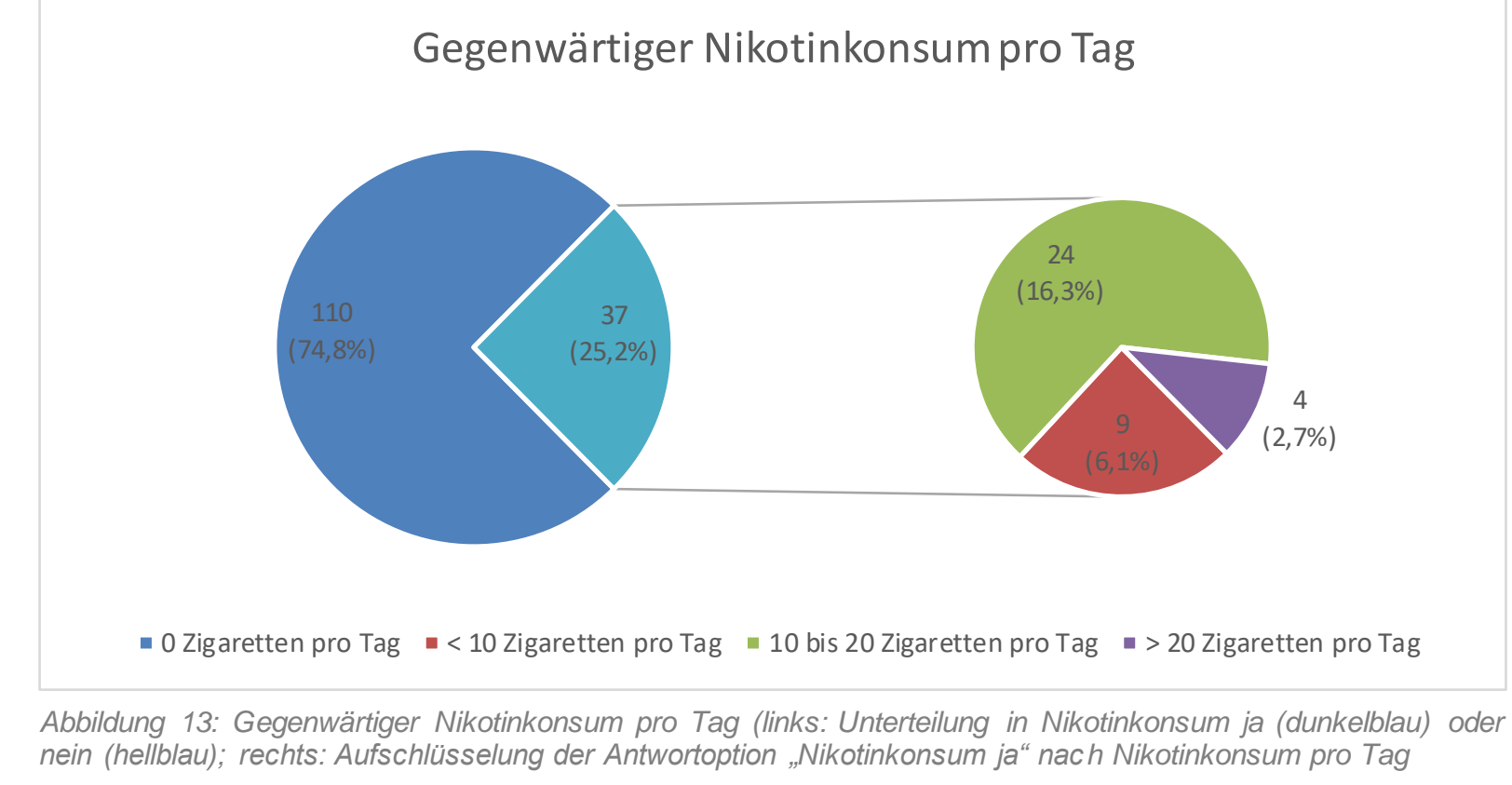

Von den insgesamt 147 männlichen Patienten waren zur Zeit der Erstaufnahme 110 (74,8\%) Nichtraucher und 37 (25,2\%) Raucher. Die Gruppe der Raucher ließ sich anhand des pro Tag Nikotinkonsums weiter unterteilen. Die meisten rauchenden Patienten (24 der 147 Patienten $(16,3 \%))$ gaben an 10 bis 20 Zigaretten pro Tag zu konsumieren. Die wenigsten Patienten in der Gruppe der Raucher (2,7\%) gaben an mehr als 20 Zigaretten pro Tag zu rauchen.

\subsubsection{Orale Glukokortikosteroide}

In die Auswertung der Einnahme oraler Glukokortikosteroide flossen sowohl die Tagesdosierung als auch der Einnahmezeitraum ein. Sofern ab dem Zeitpunkt der Erstaufnahme für die nächsten drei Monate oder in den zurückliegenden drei Monaten eine Therapie mit oralen Glukokortikosteroiden stattgefunden hat, wurde dieser Parameter als „ja“ verschlüsselt.

Bei den 147 männlichen Patienten des Studienkollektivs zeigte sich anhand der oben angeführten Kriterien, dass bei 119 der 147 (80,95\%) keine zurückliegende, derzeitige oder geplante Glukokortikoidtherapie zum Zeitpunkt der Erstaufnahme bestand. 28 der 147 (19,04\%) Patienten nahmen zum Zeitpunkt der Untersuchung orale Glukokortikoide ein. 21 (14,29\%) der Patienten nahmen orale Glukokortikoide in einer Dosierung über $5 \mathrm{mg}$ pro Tag ein, 13 $(8,8 \%)$ Patienten erhielten eine Dosierung an Prednisolon oder Prednisolonäquivalent über 7,5 mg pro Tag über eine Zeitspanne von mindestens drei Monaten. 


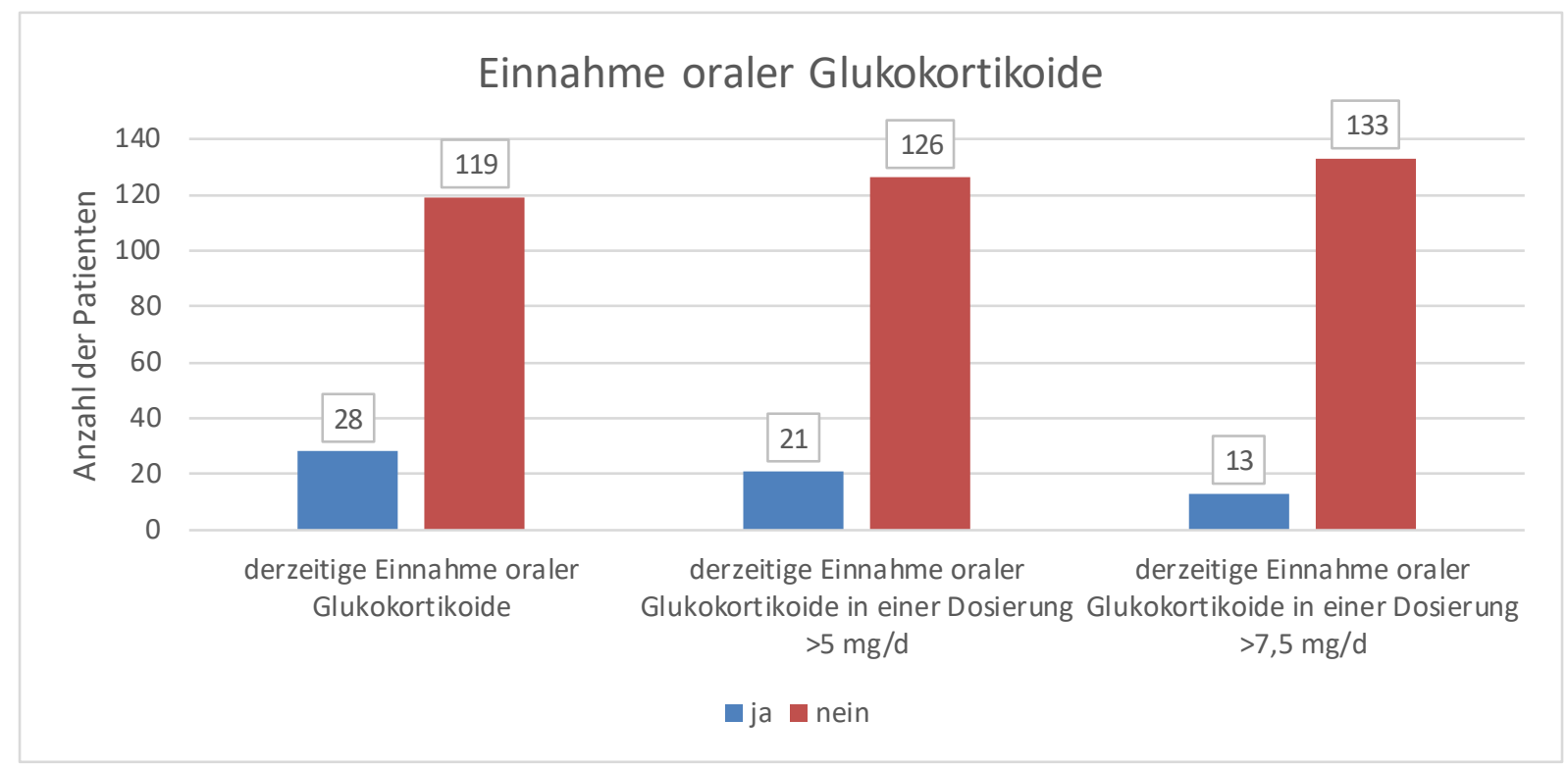

Abbildung 14: Angaben zur Einnahme oraler Glukokortikoide (unterteilt nach Dosierungen mit oralen Glukokortikoiden)

\subsubsection{Rheumatoide Arthritis}

Zum Zeitpunkt der Erstaufnahme lag basierend auf den Angaben aus den Arztbriefen bei fünf männlichen Patienten (3,4\%) eine Rheumatoide Arthritis vor, bei den übrigen 142 (96,6\%) nicht.

\section{Rheumatoide Arthritis}

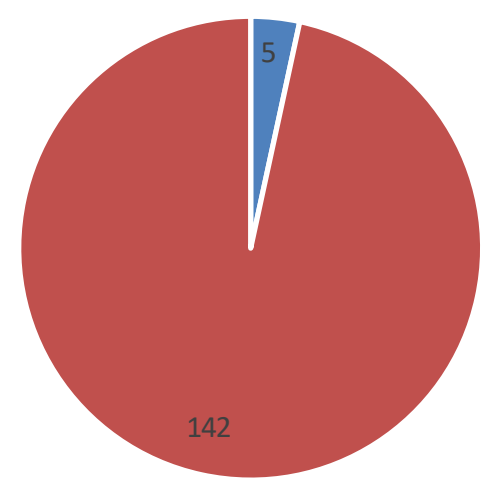

- ja - nein

Abbildung 15: Rheumatoide Arthritis

\subsubsection{Sekundäre Osteoporoseursachen}

Alle Patientendatendaten wurden bezüglich dem Vorliegen von sekundären Osteoporoseursachen analysiert. Es wurde eine Unterteilung in 23 Subgruppe bei der Auswertung vorgenommen. Die einzelnen Diagnoseschlüssel sind in der Tabelle 11 abgebildet. 
Tabelle 11: Diagnoseverschlüsselung der sekundären Osteoporosen

\begin{tabular}{ll}
\hline Diagnoseschlüssel & Sekundäre Osteoporoseursachen \\
\hline $\mathbf{1}$ & Sekundärer Hyperparathyreodismus \\
\hline $\mathbf{2}$ & Gastrointestinale Erkrankungen, Morbus Crohn \\
\hline $\mathbf{4}$ & Alkoholinduzierte Osteoporose \\
\hline $\mathbf{5}$ & Steroidinduzierte Osteoporose \\
\hline $\mathbf{6}$ & Endokrinopathien \\
\hline $\mathbf{7}$ & Hyperkalzurie \\
\hline $\mathbf{8}$ & Antiepileptika \\
\hline $\mathbf{9}$ & Thyroxin-Einnahme \\
\hline $\mathbf{1 0}$ & Hypokalzurie \\
\hline $\mathbf{1 1}$ & Primärer Hyperparathyreodismus \\
\hline $\mathbf{1 2}$ & Genetik \\
\hline $\mathbf{1 3}$ & Hypogonadismus \\
\hline $\mathbf{1 4}$ & Osteogenesis imperfecta \\
\hline $\mathbf{1 5}$ & Zustand nach Transplantation \\
\hline $\mathbf{1 6}$ & Schwangerschaft \\
\hline $\mathbf{1 7}$ & Leberzirrhose \\
\hline $\mathbf{1 8}$ & Hyperthyreose \\
\hline $\mathbf{1 9}$ & Rheumatoide Arthritis \\
\hline $\mathbf{2 0}$ & Metastasen \\
\hline $\mathbf{2 1}$ & Vitamin D-Mangel \\
\hline $\mathbf{2 3}$ & Lokalisierte Osteoporose \\
\hline & Heparin/Marcumar- Einnahme \\
\hline
\end{tabular}

Von den insgesamt 147 männlichen Patienten lag bei 79 (53,74\%) nach der oben beschrieben Einteilung kein Anhalt für eine sekundäre Osteoporose vor, bei den restlichen 68 (46,26\%) lag eine Anhalt für eine sekundäre Osteoporose vor. Untergliederte man die 68 Patienten mit einem Anhalt für eine sekundärere Osteoporose nach ihren Osteoporoseursachen, so ergab sich das in der folgenden Abbildung 16 dargestellte Bild: 


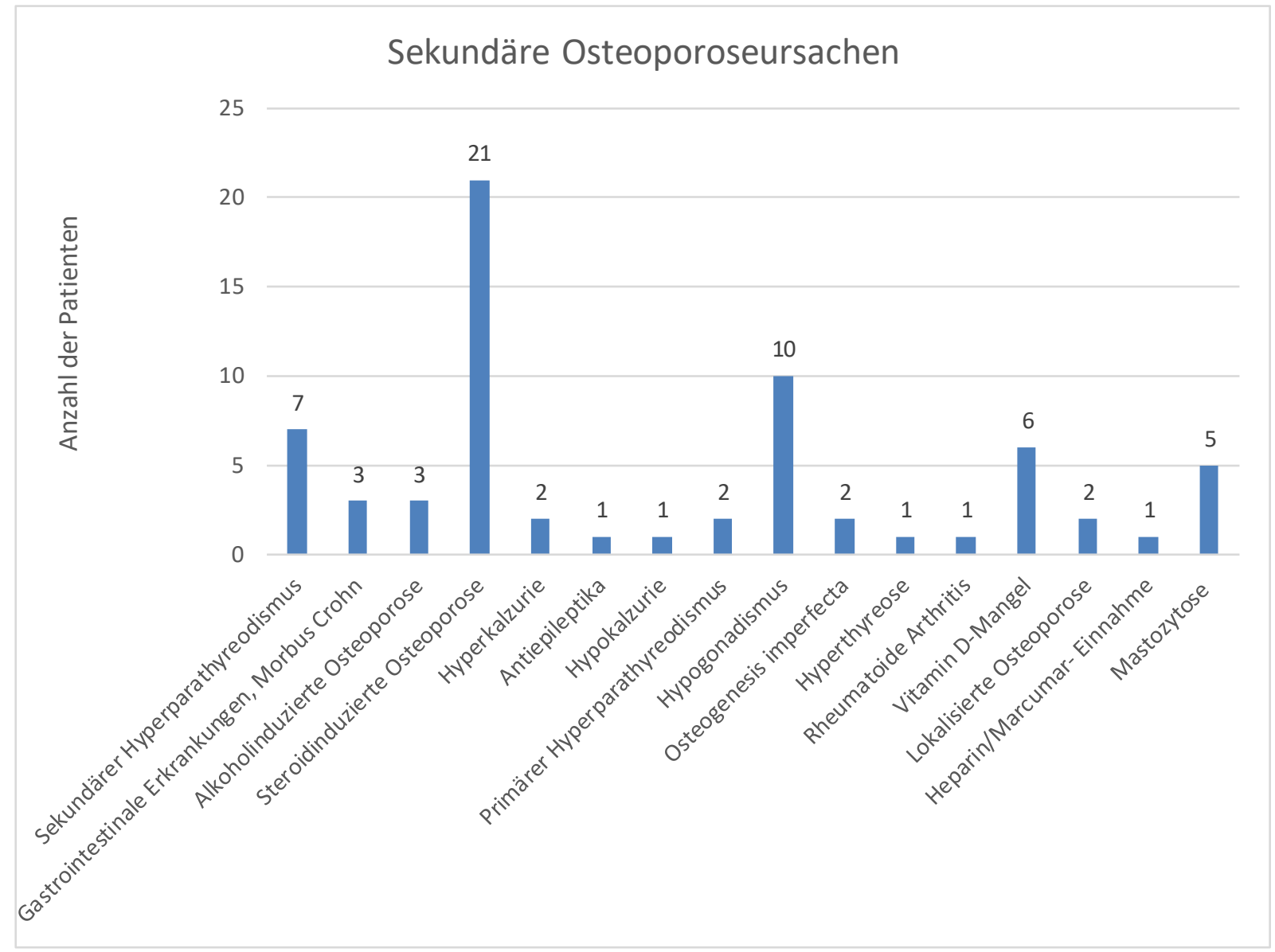

Abbildung 16: Häufigkeitsverteilung der sekundären Osteoporoseursachen

Die zahlenmäßig größte Unterform der sekundären Osteoporoseursachen stellte die der steroidinduzierten Osteoporose mit 21 (14,29\%) Patienten dar. Darauf folgte das Subkollektiv der Patienten mit einem Hypogonadismus dem zehn (15,6\%) Probanden zuzuordnen waren. Die nächstgrößeren Unterformen der Osteoporose waren die des sekundären Hyperparathyreodismus (10,9\%), des Vitamin D-Mangels (9,4\%) und der Mastozytose (7,8\%). Gar kein Patient konnte den sekundären Osteoporoseformen der Endokrinopathien $>2$ Faktoren, Thyroxin, Genetik, Transplantation, Leberzirrhose und Metastasen zugeordnet werden. Nur je einen Patienten fassten die Gruppen der Antiepileptka, Hypocalcurie, Hyperthyreose, Rheumatoide Arthritis, lokalisierte Osteoporose, zwei Patienten fassen die Untergruppen des primärer Hyperparathyreodismus, der Osteogenesis imperfecta und Heparin/Marcumar.

Basierend auf dem in der weiteren Auswertung angeführten FRAX-Score wurden die folgenden Krankheitsbilder unter dem Aspekt sekundäre Osteoporoseursachen mit „ja“ berücksichtigt: Typ I (insulinabhängiger) Diabetes, Osteogenesis imperfecta bei Erwachsenen, langjährige, unbehandelte Hyperthyreose, Hypogonadismus oder frühe Menopause (<45 Jahren), chronische Mangelernährung oder Malabsorption und chronische Lebererkrankungen. Bei insgesamt 23 Patienten (15,65\%) konnte unter Berücksichtigung dieser Punkte eine sekundäre Osteoporose diagnostiziert werden. 
Für den „FRAX+“-Score, welcher alle der in der Tabelle 11 gelisteten sekundären Osteoporoseursachen umfasst, zeigte sich, dass bei 46 Patienten $(31,29 \%)$ ein Anhalt für eine sekundäre Osteoporose vorlag. Nicht berücksichtigt wurden in diesem Fall Patienten mit den Diagnoseschlüsseln „Glukokortikosteroide“, „Rheumatoide Arthritis“, sowie „Alkohol 3 und mehr Einheiten/Tag", da diese Faktoren sonst doppelt im FRAX-Rechner (sowohl als eigenes Item als auch als sekundäre Osteoproseursache) gewertet wurden.

\subsubsection{Alkoholkonsum}

Der osteoporosespezifische Fragebogen erlaubte quantitative Angaben zum täglichen Alkoholkonsum. In Form einer vierstufigen Skala konnte jeder Patient zwischen den Angaben „kein Alkohol“, „gelegentlicher Alkoholkonsum“, „unter 2/4 Wein“ und „über 2/4 Wein“ wählen. Die Einheit „2/4 Wein“ entsprach dabei einem 2/4 Liter Wein und war somit gleichzusetzten mit $500 \mathrm{ml}$ Wein oder äquivalenten Mengen an alkoholischem Gehalt anderer Getränke (FRAXEingabemaske 2016).

\section{Alkoholeinheiten pro Tag pro Patient}

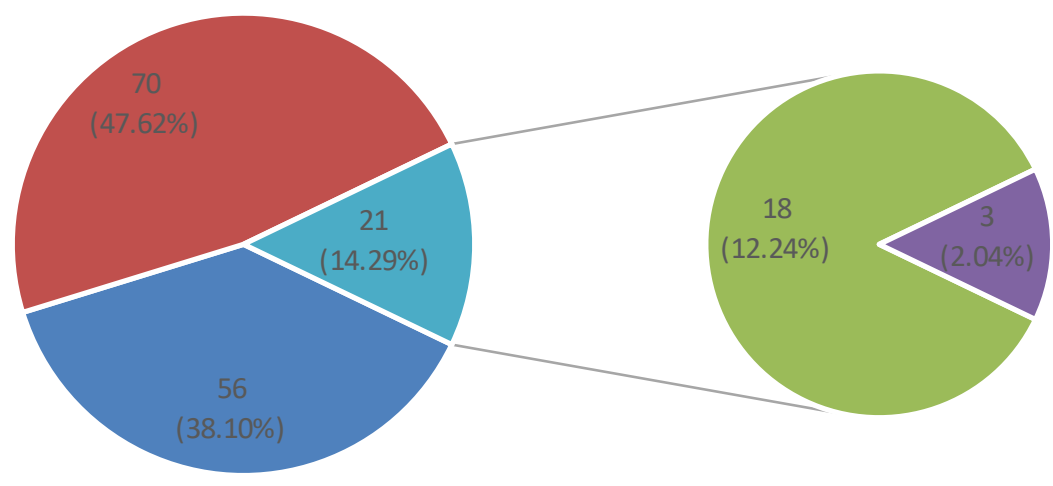

- kein Alkoholkonsum gelegentlicher Alkoholkonsum - bis 2/4 Wein über 2/4 Wein

Abbildung 17: Alkoholeinheiten pro Tag pro Patient (links: Unterteilung in kein, gelegentlicher Alkoholkonsum und regelmäßiger Alkoholkonsum; rechts: Unterteilung des regelmäßigen Alkoholkonsums in bis zu und über 2/4 Wein pro Tag)

In der Auswertung wählten 70 von 147 (47,62\%) Patienten einen „gelegentlichen Alkoholkonsum“ als zutreffende Aussage. Weitere $56(38,10 \%)$ gaben an, keinen Alkohol zu trinken. Von den übrigen 21 Patienten (14,29\%) gaben drei (2,04\%) an, täglich mehr als ein Äquivalent von 2/4 Wein an alkoholischen Getränken zu sich zu nehmen, 18 (12,24\%) verzehrten „bis zu 2/4 Wein“ pro Tag.

Transformierte man die vorliegenden Daten aus dem osteoporosespezifischen Fragebogen in die für den FRAX-Rechner verwendete Alkoholeinheit, so entsprach ein Alkoholkonsum von über drei Einheiten pro Tag etwa $360 \mathrm{ml}$ Wein pro Tag. Für die Differenzierung zwischen „mehr 
als drei Einheiten Alkohol ja oder nein“ konnte aus den vorliegenden patientenbezogenen Angaben nur eine näherungsweise Aussage getroffen werden. Die Aussage „kein Alkohol“ und „gelegentlicher Alkoholkonsum“ wurden als „unter 3 Alkoholeinheiten pro Tag“ gewertet. Die Angabe „bis 2/4 Wein“ wurde als „über drei Einheiten Alkohol“ pro Tag interpretiert, da sie einen Alkoholkonsum von bis zu $500 \mathrm{ml}$ Wein pro Tag oder äquivalenten Mengen andere alkoholischer Getränke umfasste. Dabei bestand die Möglichkeit, dass Patienten, die unter 360 ml Wein pro Tag tranken, fälschlicherweise dieser Gruppe zugeordnet wurden. Dies ließ sich basierend auf den vorliegenden Angaben nicht umgehen. Nur bei der Auswahlmöglichkeit „über 2/4 Wein“ konnte mit Sicherheit davon ausgegangen werden, dass alle diese Patienten mindestens 3 Einheiten Alkohol pro Tag konsumierten. Basierend auf dieser Modifikation zeigte sich, dass 126 Probanden (85,72\%) weniger als „3 Einheiten Alkohol pro Tag“ und 21 Probanden (14,29\%) mehr als „3 Einheiten Alkohol pro Tag“ zu sich nahmen.

\subsubsection{DXA-Messwerte}

Für insgesamt 140 der 147 Patienten lagen DXA-Messungen vor bzw. wurden zur weiteren Diagnostik zeitnah erstellt. Von den 140 Patienten mit vorliegenden DXA-Messungen, wurden 64 Knochendichtemessungen in dem medizinischen Versorgungszentrum MVZ endokrinologicum Göttingen angefertigt, die übrigen 76 Aufnahmen in externen Praxen.

Von den 140 erstellten DXA-Aufnahmen erhielten 130 vollständige Messdaten, d.h. Knochendichtemessungen an der Lendenwirbelsäule, dem Gesamtfemur und dem Schenkelhals mindestens einer Seite. Die übrigen zehn Datensätze enthielten keine Knochendichtemessungen der Schenkelhälse und konnten folglich nicht in den computerbasierten FRAX-Rechner unter Einbeziehung der Knochendichte der Schenkelhälse eingegeben werden. Von sechs dieser zehn Patientendatensätzen mit unvollständigen DXA-Messungen lagen zu den an der Wirbelsäule gemessenen Werten auch Knochendichte des Gesamtfemurs mindestens einer Seite vor, für die übrigen vier nur gemessene Werte an der Lendenwirbelsäule.

Betrachtete man die minimalen T-Scores der 130 Femurhalsmessungen, so zeigte sich eine Normalverteilung der Daten. Der gemessene Mittelwert lag bei - 2,1 mit einer Standardabweichung von $\pm 1,0$. Der niedrigste gemessene Wert lag bei $-5,2$, der höchste T-Score bei 0,6. 

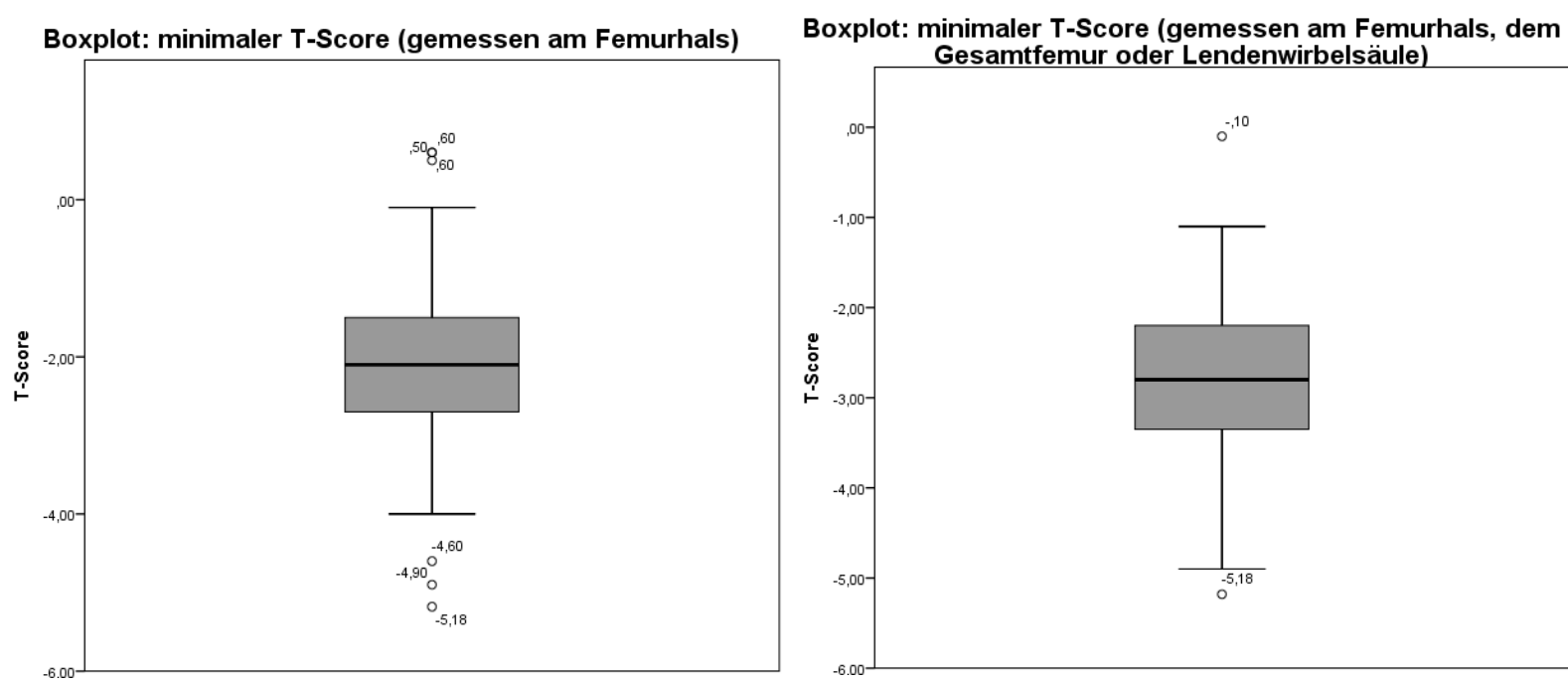

Abbildung 18: Boxplots: Minimal gemessener T-Score am Femurhals bzw. Femurhals, Gesamtfemur oder Lendenwirbelsäule ( $n=130)$

Betrachtete man den minimalen T-Score der 130 männlichen Patienten, diesmal unter Einbeziehung der gemessenen Knochendichten an der Lendenwirbelsäule und des Gesamtfemurs, so ergaben sich leicht abweichende Werte der gemittelten minimalen T-Scores. Es errechnete sich ein Mittelwert von - 2,8 mit einer Standardabweichung von $\pm 0,9$. Der niedrigste gemessene Wert lag bei einem T-Score von $-5,2$, der höchste bei einem T-Score von 0,1 . Insgesamt zeigte sich auch hier eine Normalverteilung der minimalen T-Scores.

Die Boxplots in Abbildung 18 veranschaulichen die Verteilung der minimal gemessenen Knochendichten. 50\% der minimal gemessenen Knochendichtendichten am Femurhals lagen zwischen einem T-Score von 0,6 und -1,5. Betrachtete man die minimal gemessenen T-Scores an allen Messorten, so lagen 50\% der Werte zwischen -0,1 und -2,2.In Anlehnung an die WHO ist die Diagnose einer Osteoporose zulässig, sofern ein T-Score von - $\leq 2,5$ an der Lendenwirbelsäule, dem Gesamtfemur oder dem proximalen Femurhals gemessen wird (WHO 1991). Im untersuchten Kollektiv mit vollständigen Knochendichtemessungen zeigte sich, dass bei $33,84 \%$ der Patienten ein T-Score $\leq-2,5$ am Schenkelhals vorlag, bei 48,46\% ein T-Score von $\leq-2,5$ an der Lendenwirbelsäule. Betrachtete man die minimal gemessenen T-Scores, so lag bei $63,84 \%$ ein T-Score $\leq-2,5$ vor.

\subsection{0-Jahres-Frakturrisiko nach den DVO-Leitlinien aus den Jahren 2006, 2009 und 2014}

Basierend auf den Datenquellen war eine Unterteilung des Patientenkollektivs in drei 10-Jahres-Frakturrisisikogruppen möglich: $<20 \%, 20-30 \%$ und $>30 \%$. Die meisten Patienten, $61,9 \%$ (91 Patienten), hatten ein über 30\%iges Risiko für das Auftreten eines osteoporoseassoziierten Bruches in den kommenden zehn Jahren. Bei 48 dieser Patienten bestand ein Anhalt für eine primäre Osteoporose, bei 43 Patienten ein Anhalt für eine sekundäre Osteoporose. In 
den übrigen Gruppen reduzierte sich mit der Abnahme des Frakturrisikos auch die Anzahl der zugeordneten Patienten. So hatten 38 von 147 Patienten (25,85\%) ein 10-Jahres-Frakturrisiko zwischen 20 und 30\%, 18 Patienten (12,24\%) ein unter 20\%iges 10-Jahresrisiko. Auf einem Konfidenzintervall von einem Prozent, zeigte sich, dass die Daten nicht normalverteilt sind.

10-Jahres-Frakturrisiken basierend auf den DVO-Leitlinen aus den Jahren 2006-2014 (unterteilt in primäre und sekundäre Osteoporose)

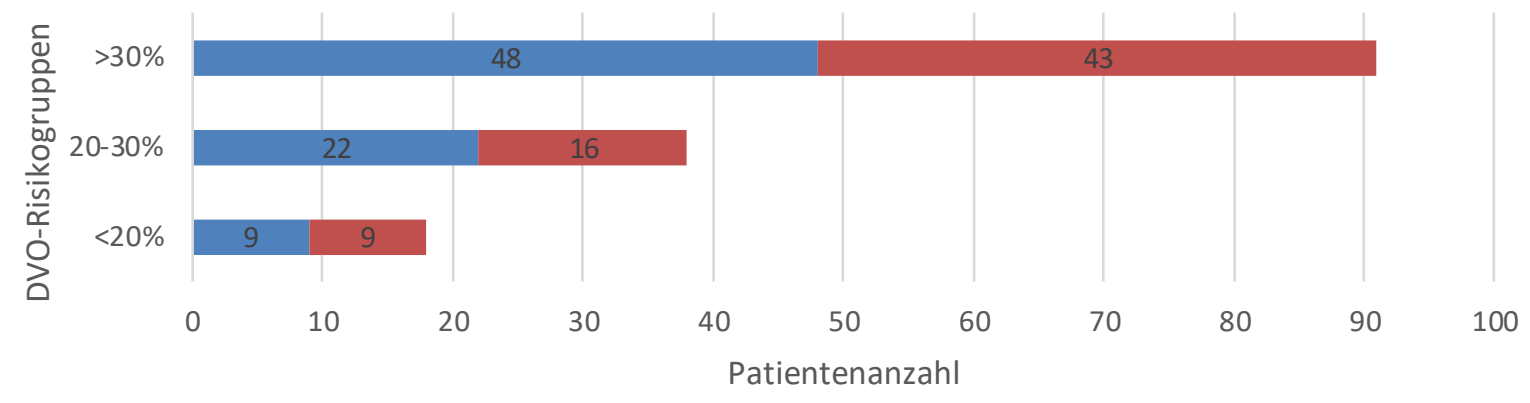

anahlt für eine primäre Osteoporose $\quad$ Anhalt für eine sekundäre Osteoporose

Abbildung 19: Anzahl der Patienten gruppiert nach prozentualen 10-Jahres Frakturrisiken basierend auf den DVOLeitlinien aus den Jahren 2006, 2009 und 2014, unterteil in primäre (blau) und sekundäre (rot) Osteoporoseursachen

\subsection{FRAX- und „FRAX+"- Risikomodelle}

Das individuelle 10-Jahres-Frakturrisiko für eine MOF, sowie eine HF ließ sich basieren auf dem im MVZ endokrinologikum Göttingen erhobenen Datensatz für 147 (ohne BMD) bzw. für 130 (mit BMD) berechnen. Tabelle 12 zeigt eine Zusammenfassung der deskriptiven Statistiken der beiden FRAX-Modelle für die unterschiedlichen Frakturtypen sowohl ohne als auch mit Einbeziehung der Knochendichtemessung am Schenkelhals.

Die höchsten Mittelwerte wurden für den „FRAX+“-Score errechnet. Verglich man die Mittelwerte aller FRAX-Modelle, so zeigte sich, dass die im Durchschnitt errechneten 10-JahresFrakturrisiken für das Auftreten einer HF niedriger als die entsprechenden Werte einer MOF lagen. Zudem zeigte sich, dass die Risiken nochmals geringere prozentuale Werte annahmen, sofern man die Knochendichtemessung nicht miteinbezog. Die höchsten Mittelwerte errechneten sich jeweils durch den „FRAX+“-Score. Unter Integration der BMD errechnete sich zwischen dem FRAX-Score und dem „FRAX+“-Score identische Mittelwerte. 
Tabelle 12: Deskriptive Statistik FRAX-und „FRAX+"-10-Jahres-Fralturrisiken

\begin{tabular}{|c|c|c|c|c|c|c|c|c|}
\hline \multirow[t]{3}{*}{ Deskriptive Statistik } & \multicolumn{4}{|c|}{$\begin{array}{c}\text { FRAX-10-Jahres-Frakturrisiken in } \\
\%\end{array}$} & \multicolumn{4}{|c|}{$\begin{array}{c}\text { „FRAX+“-10-Jahres-Frakturrisiken } \\
\text { in } \%\end{array}$} \\
\hline & MOF & HF & MOF & HF \% & „MOF & HF & MOF & HF \\
\hline & \multicolumn{2}{|c|}{ Ohne BMD } & \multicolumn{2}{|c|}{ Mit BMD } & \multicolumn{2}{|c|}{ Ohne BMD } & \multicolumn{2}{|c|}{ Mit BMD } \\
\hline $\mathbf{n}$ & 147 & 147 & 130 & 130 & 147 & 147 & 130 & 130 \\
\hline Mittelwert & 6,2 & 1,97 & 11,8 & 6,8 & 6,62 & 2,24 & 11,8 & 6,8 \\
\hline Median & 5,0 & 1,1 & 8,5 & 3,35 & 5,3 & 1,1 & 8,5 & 3,35 \\
\hline Standardabweichung & 3,75 & 2,5 & 11,03 & 10,6 & 4,36 & 2,97 & 11,03 & 10,6 \\
\hline Minimum & 1,8 & 0,1 & 2,1 & 0,1 & 1,9 & 0,1 & 2,1 & 0,1 \\
\hline Maximum & 20 & 15 & 85 & 84 & 28 & 21 & 85 & 84 \\
\hline Normalverteilung & Nein & Nein & Nein & Nein & Nein & Nein & Nein & Nein \\
\hline
\end{tabular}

Bestimmte man mittels Kendall-tau-b die Korrelationen der einzelnen FRAX- und „FRAX+“Scores untereinander, so ergaben sich die in der Kreuztabelle (Tabelle 13) abgebildeten Korrelationskoeffizienten.

Tabelle 13: Korrelationskoeffizienten der FRAX-und "FRAX+"-Scores ( ${ }^{* *}$ Signifikanzniveau $\left.p=0,01\right)$

\begin{tabular}{|c|c|c|c|c|c|c|c|c|c|c|}
\hline \multirow{2}{*}{\multicolumn{3}{|c|}{$\begin{array}{l}\text { Korrelationskoeffizien- } \\
\text { ten }(r)\end{array}$}} & \multicolumn{4}{|c|}{ FRAX-Score } & \multicolumn{4}{|c|}{ „,FRAX+“"Score } \\
\hline & & & \multicolumn{2}{|c|}{ Ohne BMD } & \multicolumn{2}{|c|}{ Mit BMD } & \multicolumn{2}{|c|}{ Ohne BMD } & \multicolumn{2}{|c|}{ Mit BMD } \\
\hline & & & MOF & HF & MOF & HF & MOF & HF & MOF & HF \\
\hline \multirow{4}{*}{ 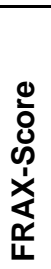 } & \multirow{2}{*}{ 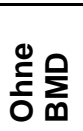 } & MOF & & & & & & & & \\
\hline & & HF & $0,786^{\star \star}$ & & & & & & & \\
\hline & \multirow{2}{*}{$+\frac{Q}{\Sigma}$} & MOF & $0,47^{* *}$ & $0,396^{* *}$ & & & & & & \\
\hline & & HF & $0,344^{\star \star}$ & $0,352^{* *}$ & $0,82^{\star *}$ & & & & & \\
\hline \multirow{4}{*}{ 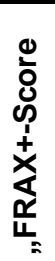 } & \multirow{2}{*}{$\stackrel{Ð}{\stackrel{\Phi}{c}}$} & MOF & $0,891^{\star \star}$ & $0,736^{\star *}$ & $0,467^{\star \star}$ & $0,347^{\star \star}$ & & & & \\
\hline & & HF & $0,747^{\star \star}$ & $0,920^{\star *}$ & $0,397^{\star *}$ & $0,353^{\star \star}$ & $0,777^{* *}$ & & & \\
\hline & \multirow{2}{*}{$+\sum_{m}^{0}$} & MOF & $0,47^{\star *}$ & $0,396^{* *}$ & $1^{* *}$ & $0,82^{* *}$ & $0,467^{\star *}$ & $0,397^{\star *}$ & & \\
\hline & & HF & $0,344^{\star \star}$ & $0,352^{\star *}$ & $0,82^{\star *}$ & $1^{* *}$ & $0,347^{* *}$ & $0,353^{* *}$ & $0,82^{* *}$ & \\
\hline
\end{tabular}

Zwischen den FRAX-Scores zeigte sich auf einem Signifikanzniveau von einem Prozent eine positive Korrelation. Die stärkste Korrelation $(r=0,82)$ bestand zwischen dem FRAX-Score für die Prognose einer MOF (mit BMD) und dem FRAX-Score einer HF (mit BMD). Dies bedeutet, dass die zusätzliche Eingabe der ausgewählten Risikofaktoren bei gleichzeitiger Eingabe der BMD keinen zusätzlichen Effekt auf das Frakturrisiko hat. Die schwächste Korrelation zeigte sich zwischen dem FRAX-Score zur Vorhersage einer MOF (ohne BMD) und dem FRAX- 
Score einer HF (mit BMD). Im Wilcoxon-Test ergab sich zwischen den FRAX-Scores untereinander auf einem Signifikanzniveau von einem Prozent ein hoch signifikanter Unterschied der zentralen Aussagetendenz.

Auch zwischen allen „FRAX+“-Scores zeigte sich eine positive Korrelation auf einem Signifikanzniveau von einem Prozent. Die im Vergleich stärkste Korrelation bestand zwischen dem „FRAX+“-Score für das 10-Jahres-Risiko einer HF und dem „FRAX+“-Score einer MOF jeweils ohne Einbeziehung der gemessenen T-Scores $(r=0,891)$. Diese beiden „FRAX+“-Scores stimmten folglich am besten bezüglich ihrer 10-Jahres-Frakturrisiken überein. Die schwächste Korrelation zeigte sich zwischen dem „FRAX+“-Score für eine MOF ohne Einbeziehung der Knochendichte und dem „FRAX+“-Score für eine HF mit Einbeziehung der DXA-Messung $(r=0,346)$. So zeigte sich, dass die Einbeziehung der BMD zu deutlichen Unterschieden in den 10-Jahres-Frakturrisiken führte, die am stärksten hinsichtlich der Lokalisationen (MOF oder HF) variierten. Für alle FRAX+“-Scores zeigte sich im Wilcoxon-Test auf einem Signifikanzniveau von einem Prozent ein signifikanter Unterschied der zentralen Aussagetendenz. Demnach unterschieden sie sich die prognostizierten 10-Jahres-Frakturrisiken der einzelnen „FRAX+"-Scores hochsignifikant voneinander in der hier untersuchten männlichen Population. Verglich man die einzelnen FRAX- und „FRAX+“-Scores durch eine Korrelation nach Kendalltau b, so bestand auf einem Signifikanzniveau von einem Prozent eine positive Korrelation zwischen aller FRAX- und „FRAX+“-Scores. Sowohl für das Ereignis einer MOF als auch einer HF zeigte sich unter Einbeziehung der Knochendichtemessung ein Korrelationskoeffizient von 1 zwischen dem FRAX- und „FRAX+“-Score. Sie errechneten jeweils patientenbezogene identische 10-Jahres-Frakturrisiken. Zwischen den restlichen FRAX- und „FRAX+“-Scores zeigte sich trotz der positiven Korrelation $(p=0,01)$ im Wilcoxon-Test ein Unterschied der zentralen Aussagetendenzen $(p=0,01)$. Demnach unterschieden sich die prognostizierten 10-JahresFrakturrisien hochsignifikant sowohl in Abhängigkeit des gewählten Sets an Risikofaktoren als auch der beabsichtigen Frakturttypenprognose (MOF oder HF).

\subsubsection{Therapieempfehlungen basierend auf den FRAX- Risikomodellen}

Im direkten Vergleich verschiedener länderspezifischen Osteoporoseleitlinien und wissenschaftlicher Arbeiten wird ein Frakturrisiko $\geq 20 \%$ für eine MOF bzw. $\geq 3 \%$ für eine HF als häufigste Interventionsgrenze beschrieben (Kanis et al. 2016a). Zusätzlich wird die in den Leitlinien des DVO empfohlene Therapieschwelle von $\geq 14 \%$ für eine MOF angegeben. Unter Verwendung dieser Therapiegrenzen ergaben sich für das untersuchte Patientenkollektiv die in der Tabelle 14 dargestellten Ergebnisse. 
Tabelle 14: Therapieempfehlungen ,ja" basierend auf den FRAX-Modellen

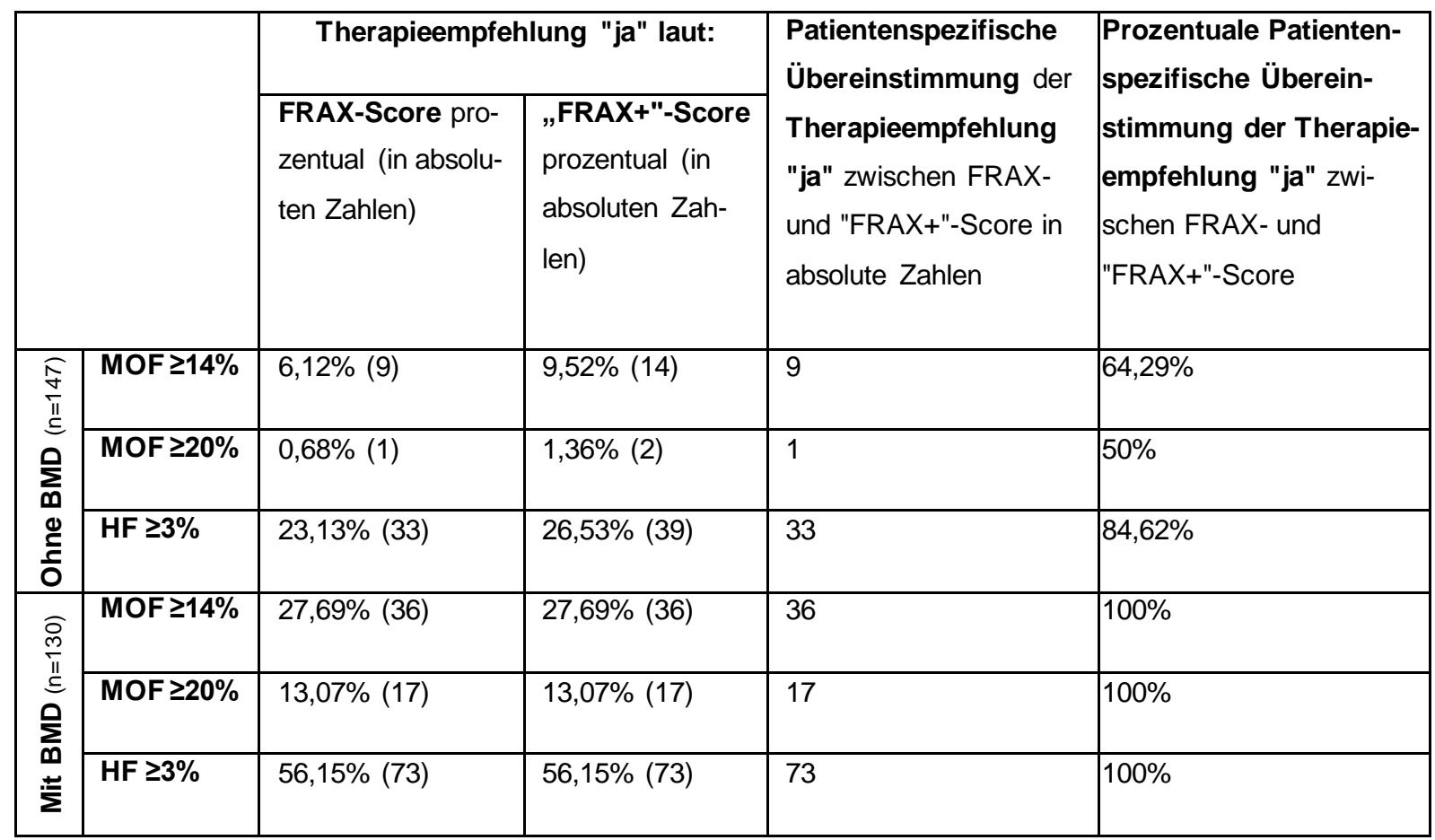

Es zeigte sich, dass der „FRAX+“-Score ohne BMD im Vergleich zum FRAX-Score ohne BMD mehr Patienten als therapiebedürftig identifizierte. Für das Ereignis einer MOF ohne BMD wurden vom FRAX-Score in Abhängigkeit der Therapiegrenze von $\geq 14 \%$ bzw. $\geq 20 \% 6,12 \%$ bzw. $0,68 \%$ als therapiebedürftig eingestuft. Weiteren $3,4 \%$ der Patienten (Therapiegrenze $\geq 14 \%$ ) bzw. weiteren 0,68\% (Therapiegrenze $\geq 20 \%$ ) wurde durch den „FRAX+“-Score eine Therapie empfohlen. Für das Ereignis einer HF ohne BMD zeigte sich eine Übereinstimmung in der Therapieempfehlung ,ja“ des FRAX- und „FRAX+“-Scores bei 84,62\% der Patienten. Weitere $3,4 \%$ der Patienten erhielten eine Therapieempfehlung basierend auf dem „FRAX+“-Score im Vergleich zum FRAX-Score. Für das Auftreten einer MOF, sowie einer HF mit BMD ergab sich eine Übereinstimmung der Therapieempfehlung „ja“ von 100\% (je 36 bzw. 17 bzw. 73 Patienten) zwischen dem FRAX- und „FRAX+“-Score. Folglich zeigte sich durch die zusätzliche Implikation weiterer Risikofaktoren in den „FRAX+“-Score kein Einfluss auf die Patientenbezogenen Therapieempfehlungen im Vergleich zum FRAX-Score, sofern die Knochendichtemessung miteinbezogen wurde. Ohne Verwendung der BMD wurden basierend auf dem FRAXScore weniger Patienten eine Therapie empfohlen als durch den „FRAX+“-Score. Alle Patienten, die durch den FRAX-Score als therapiebedürftig eingestuft wurden, wurden auch durch den „FRAX+“-Score als solche erkannt.

\subsection{1-Jahres-Frakturrisiken nach C. Glüer}

Basierend auf dem nach C. Glüer entwickelten Score ließ sich eine Jahresprognose für das Frakturrisiko jedes einzelnen Patienten angeben. Es zeigte sich, wie in der Abbildung 20 dargestellt, dass die meisten Patienten (36,01\%) ein 0 bis $1 \%$ iges 1 -Jahres-Frakturrisiko hatten. 
Die zweitstärkste Gruppe fasste 32 Patienten (21,77\%), welche alle ein 1 bis $2 \%$ iges Risiko hatten in dem nächsten Jahr eine osteoporoseassoziierte Fraktur zu erleiden. Ab einem $4 \%$ igen Frakturrisiko zeigten sich durchgehend deutlich kleinere Gruppenstärke, welche zwischen vier und neun Patienten fassten.

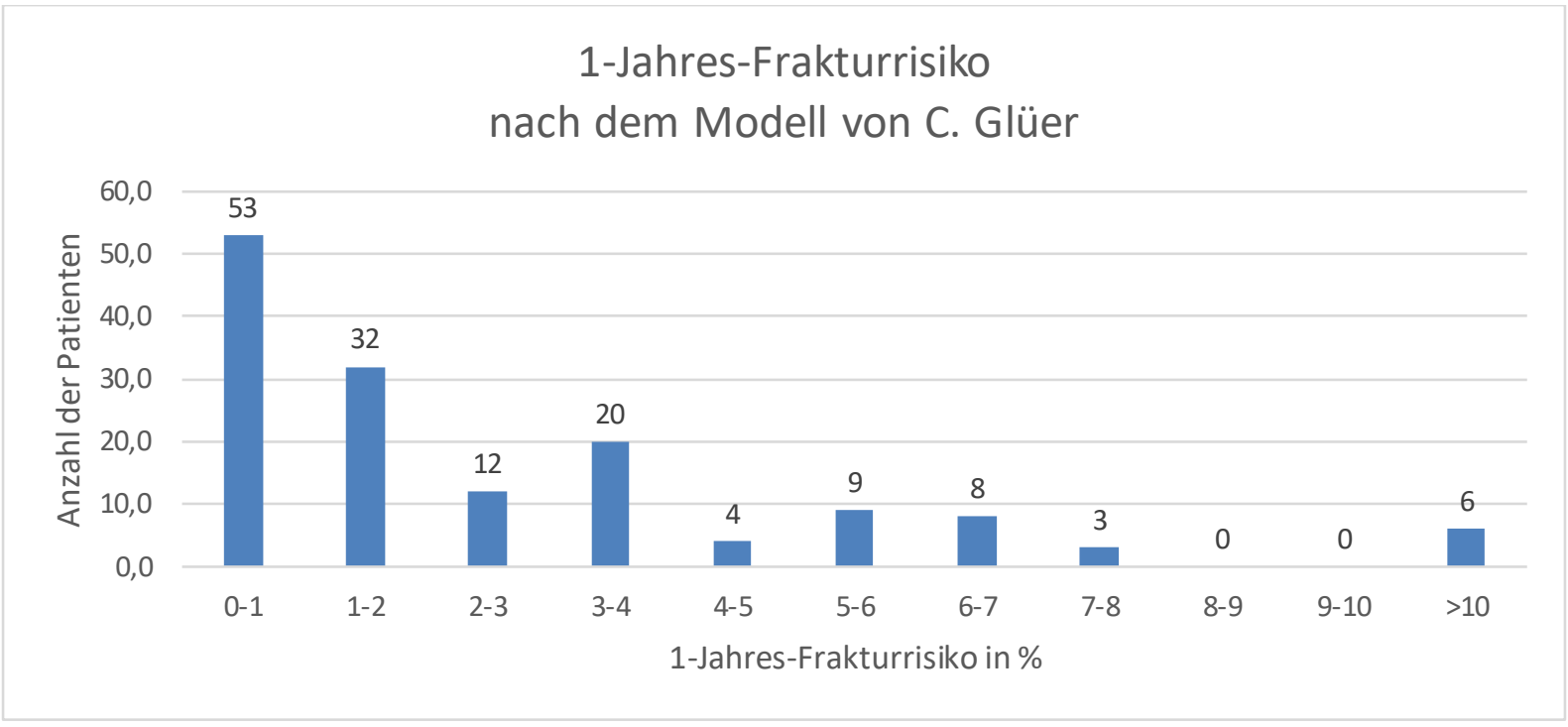

Abbildung 20:1-Jahres-Frakturrisiko nach dem Modell von C. Glüer (n=147)

Im Schnitt hatten die Patienten ein 1-Jahres-Frakturrisiko von 2,55\%, mit einer Standardabweichung von 2,46\%. Der Median lag bei 1,5\%. Es zeigte sich keine Normalverteilung der Frakturrisiken $(p=0,01)$.

\subsubsection{Therapieempfehlungen basierend auf dem Score nach C. Glüer}

Ausgehend von dem errechneten 1-Jahres-Frakturrisiko ließ sich eine Aussage treffen, ob eine osteoporosespezifische Therapie empfohlen werden sollte. Als absolute Therapieindikation galt ein 1-Jahres-Frakturrisko über $6 \%$. Sofern der gemessene T-Score unter - 2 Standardabweichungen lag, sollte eine spezielle Therapie bereits ab einem 1-Jahres-Frakturrisiko $>3 \%$ begonnen werden.

Ausgehend von dieser Definition konnte bei 33 der 147 Patienten $(22,43 \%)$ eine klare Therapieempfehlung ausgesprochen werden. 113 Patienten $(76,87 \%)$ wurde keine Therapie empfohlen. Nur für einen Patienten konnte keine eindeutige Entscheidung getroffen werden, da dieser ein Frakturrisiko von 5,2\%, jedoch keine Knochendichtemessung hatte. Um ihn in der weiteren Auswertung berücksichtigen zu können, wurde er zu der Gruppe derjenigen Patienten gezählt, die keine Therapieempfehlung erhielten. Bezog man sich nur auf die 130 männlichen Patienten, für die eine Knochendichtemessung am Schenkelhals vorlag und welche somit im FRAX-Score unter Einbeziehung der Knochendichtemessung berücksichtigt wurden, so wurde für 29 der 130 Patienten (22,3\%) eine positive Therapieempfehlung ausgesprochen. 


\subsubsection{Hochrechnung des 1-Jahres-Frakturrisiko auf ein 10-Jahres-Frakturrisiko}

Im Hinblick auf die bessere Vergleichbarkeit der Scores untereinander, wurde der DVO-Score nach C. Glüer mit dem Faktor zehn multiplizieren, sodass man ein näherungsweises 10-Jahres-Frakturrisiko erhielt.

Diese neu berechneten 10-Jahres-Frakturrisiken ließen sich analog zu den DVO-Leitlinien aus den Jahren 2006, 2009, 2014 und 2017 in drei Wertebereiche (<20\%, 20-30\%, >30\%) einteilen. Die größte dieser drei Gruppen fasste 85 der 147 Patienten (57,82\%). Sie alle hatten ein Frakturrisiko unter $20 \%$ in den nächsten zehn Jahren eine Fraktur zu erleiden. Ein $20-30 \%$ iges 10-Jahres-Frakturrisiko hatten 40 Patienten (27,21\%), ein über $30 \%$ iges Frakturrisiko 22 Patienten $(14,97 \%)$.

\subsection{Vergleichende Betrachtung der 10-Jahres-Frakturrisiken des DVO-, FRAX-, „FRAX+“-Scores und dem Score nach C. Glüer}

Betrachtete man den DVO-Score bezüglich der Patientenverteilung in die drei Risikobereichen $<20 \%, 20-30 \%$ und $>30 \%$, so zeigte sich ein Anstieg der Patientenanzahlen pro Risikogruppe je höher das prognostizierte 10-Jahres-Frakturrisiko lag. Der Wertebereich mit einem über 30\%igem Risiko in den nächsten 10 Jahren eine osteoporoseassoziierte Fraktur zu erleiden, fasste $61,09 \%$ der Patienten.

Für die Auswertung der analog zu den DVO-Risikobereichen angeordneten FRAX- und „FRAX+“-basierten 10-Jahres-Frakturrisiken wurden die 1-Jahresfrakturrisiken mit 10 multipliziert. Es zeigte sich die folgende Verteilung der Patienten auf die einzelnen Risikogruppen: Ein über 30\%iges Risiko einer MOF oder HF ohne BMD wurde für $0 \%$ der Patienten durch das FRAX- oder „FRAX+“-Modell prognostiziert. Unter Einbeziehung der Knochendichtemessung lag ein über 30\%iges 10-Jahresfrakturrisiko für das Ereignis einer MOF sowie einer HF für das FRAX- und „FRAX+“-Modell bei je 3,9\% der Patienten vor (im DVO-Score: 61.09\%) Das errechnete arithmetische Mittel der FRAX-Scores lag in diesem Wertebereich bei 56,0\% für eine MOF bzw. 50,8\% für eine HF (vergleiche Tabelle 15).

Ein unter 20\%iges 10-Jahres-Frakturrisiko errechnete sich im FRAX-Modell ohne Einbeziehung der Knochendichtemessung für das Ereignis einer MOF bei 99,3\%, für das Ereignis einer HF bei $100 \%$ der Patienten. Mit Einbeziehung der Knochendichtemessung ergab sich für das FRAX- und „FRAX+“-Modell ein unter 20\%iges Frakturrisiko für eine MOF bei 86,9\% der Patienten, für eine HF bei $94,62 \%$ der Patienten (im DVO-Score: 12,2\%). 
Tabelle 15: 10-Jahres-Frakturrisiken des DVO-, FRAX- und „FRAX+“-Scores sowie des Scores nach C. Glüer, angeordnet in Wertebereiche unter Angabe der Anzahl der Patienten in der jeweiligen Gruppe sowie dem prozentualen Anteil an der Gesamtzahl der Patienten (n)

\begin{tabular}{|c|c|c|c|c|c|c|}
\hline \multicolumn{3}{|c|}{ 10-Jahres-Frakturrisiken } & $\mathbf{n}$ & $\begin{array}{l}<20 \% \\
\text { (prozentual) }\end{array}$ & $\begin{array}{l}20-30 \% \\
\text { (prozentual) }\end{array}$ & $>30 \%$ \\
\hline \multicolumn{3}{|c|}{ DVO-Score } & 147 & $18(12,2 \%)$ & $38(25,9 \%)$ & $91(61,9 \%)$ \\
\hline \multirow{4}{*}{ 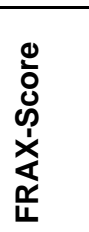 } & \multirow{2}{*}{$\stackrel{\text { }}{\text { 巳 }} \sum_{0}^{0}$} & MOF & 147 & $146(99,3 \%)$ & $1(0,7 \%)$ & $0(0,0 \%)$ \\
\hline & & HF & 147 & $147(100 \%)$ & $0(0,0 \%)$ & $0(0,0 \%)$ \\
\hline & \multirow{2}{*}{$=\sum_{m}^{O}$} & MOF & 130 & $113(86,9 \%)$ & $12(9,2 \%)$ & $5(3,9 \%)$ \\
\hline & & HF & 130 & $123(94,6 \%)$ & $2(1,5 \%)$ & $5(3,9 \%)$ \\
\hline \multirow{4}{*}{ 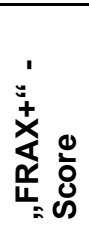 } & \multirow{2}{*}{ 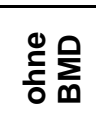 } & MOF & 147 & $146(99,3 \%)$ & $1(0,7 \%)$ & $0(0,0 \%)$ \\
\hline & & HF & 147 & $147(100 \%)$ & $0(0,0 \%)$ & $0(0,0 \%)$ \\
\hline & \multirow{2}{*}{$=\sum_{m}^{O}$} & MOF & 130 & $113(86,9 \%)$ & $12(9,2 \%)$ & $5(3,9 \%)$ \\
\hline & & HF & 130 & $123(94,6 \%)$ & $2(1,5 \%)$ & $5(3,9 \%)$ \\
\hline \multicolumn{3}{|c|}{ Score nach C. Glüer ${ }^{\star} 10$} & 147 & $85(57,8 \%)$ & $22(15 \%)$ & $40(27,2 \%)$ \\
\hline
\end{tabular}

Für die FRAX- und „FRAX+“- basierten 10-Jahres-Frakturprognosen lagen aus dem Jahresfrakturrisiko errechnete absolute Zahlen für die Frakturrisiken jedes Patienten vor. So war es möglich denen nach dem DVO-Score zugeordneten 10-Jahres-Frakturrisiken (20\%, 20-30\%, $>30 \%$ ) die entsprechenden FRAX bzw. „FRAX+" Mittelwerte zuzuordnen. Eine Übersicht der entsprechenden Ergebnisse findet sich in der Tabelle 16

Ohne Einbeziehung der Knochendichtemessung ergab sich für alle Patienten, die laut dem DVO-Score ein 10-Jahres-Frakturrisiko $<20 \%$ hatten ein durchschnittliches FRAX-10-Jahresrisiko für eine MOF von 4,73\% $\pm 3,12$. Der parallel für die 10-Jahresprognose einer HF ohne Einbeziehung der Knochendichte berechnete Mittelwert lag bei 1,19\% $\pm 1,67$. Mit BMD errechnete sich für alle Patienten mit einem DVO-10-Jahres-Frakturrisiko von $<20 \%$ anhand des FRAX-Modells ein 10-Jahresfrakturrisiko von 6,08\% $\pm 2,7$ für eine MOF und ein Mittelwert von $1,84 \% \pm 1,16$ für eine HF: Für alle Patienten mit einem DVO-10-Jahres-Frakturrisiko $>30 \%$ errechneten sich sowohl für den FRAX-Score unabhängig von der Knochendichtemessung deutlich unter 30\%ige durchschnittliche 10-Jahres-Frakturrisiken (vergleiche Tabelle 16). Der Mittelwert des „FRAX+“-Modells für alle Patienten mit einem DVO-Score <20\% lag ohne Einbeziehung der Knochendichtemessung für das Auftreten einer MOF bei $4,86 \% \pm 3,08$, für eine Hüftfraktur bei $1,24 \% \pm 1,66$. Für alle Patienten mit einem DVO-Score $>30 \%$ lag der Mittelwert im „FRAX+“-Score für eine MOF bei 7,43\% $\pm 4,37$ und bei 2,77 $\pm 3,26$ für eine HF. Unter Einbeziehung der Knochendichte errechneten sich Mittelwert, die identisch zu denen des FRAX-Scores waren. Dies gilt in allen DVO-Risikogruppen.

Analog zu den FRAX-basierten Modellen war im Score nach C. Glüer die zahlenmäßig stärkste Risikogruppe mit 57,82\%, die Patientengruppe mit einem unter 20\%igen 10-Jahres- 
Frakturrisiko. Ein über 30\%iges 10-Jahres-Frakturrisiko hatten 27,21\% der Patienten. Anhand der verhältnisskalierten 10-Jahres-Frakturrisiken nach C. Glüer ließ sich der Mittelwert in den anhand des DVO-Scores zugeordneten Prozentbereichen errechnen. In der Risikogruppe mit einem laut DVO-Score unter 20\%igem 10-Jahres-Frakturrisiko ergab sich eine durchschnittliche Bruchwahrscheinlichkeit von 14,21\% \pm 13,55. In dem Wertebereich zwischen 20 und 30\% lag das durchschnittliche 10-Jahres-Frakturrisiko bei 17,49\% $\pm 22,19$. Die Patienten mit einem über 30\%igem Risiko in den nächsten 10-Jahren eine Fraktur zu erleiden hatten gemittelt ein Risiko von $31,02 \% \pm 25,45$.

Tabelle 16: Mittelwerte der FRAX-Scores bzw. des Scores nach C. Glüer aufgeteilt nach den jeweiligen 10-JahresFrakturrisiko Wertebereiche des DVO-Scores

\begin{tabular}{|c|c|c|c|c|c|}
\hline \multicolumn{3}{|c|}{ 10-Jahres-Frakturrisiken } & \multicolumn{3}{|c|}{ Mittelwert des absoluten 10-Jahres-Frakturrisiko \pm SD } \\
\hline & & & DVO-Score $<20 \%$ & DVO-Score $20-30 \%$ & DVO-Score $>30 \%$ \\
\hline \multirow{4}{*}{ 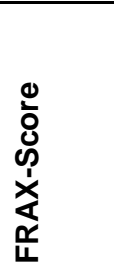 } & \multirow{2}{*}{ 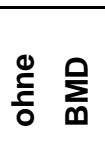 } & MOF & $4,73 \% \pm 3,12$ & $5 \% \pm 3,26$ & $6,92 \% \pm 3,79$ \\
\hline & & HF & $1,19 \% \pm 1,67$ & $1,16 \% \pm 1,54$ & $2,47 \% \pm 2,78$ \\
\hline & \multirow{2}{*}{$=\frac{O}{E}$} & MOF & $6,08 \% \pm 2,70$ & $8,64 \% \pm 5,35$ & $14,43 \% \pm 12,91$ \\
\hline & & HF & $1,84 \% \pm 1,16$ & $3,86 \% \pm 3,62$ & $9,18 \% \pm 12,77$ \\
\hline \multirow{4}{*}{ 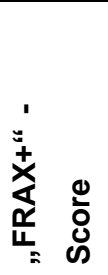 } & \multirow{2}{*}{ 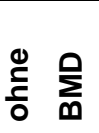 } & MOF & $4,86 \% \pm 3,08$ & $5,53 \% \pm 4,30$ & $7,43 \% \pm 4,37$ \\
\hline & & HF & $1,24 \% \pm 1,66$ & $1,42 \% \pm 2,86$ & $2,77 \pm 3,26$ \\
\hline & \multirow[b]{2}{*}{$=\frac{Q}{E}$} & MOF & $6,08 \% \pm 2,70$ & $8,64 \% \pm 5,35$ & $14,43 \% \pm 12,91$ \\
\hline & & HF & $1,84 \% \pm 1,16$ & $3,86 \% \pm 3,62$ & $9,18 \% \pm 12,77$ \\
\hline \multicolumn{3}{|c|}{ Score nach C. Glüer*10 } & $14,21 \% \pm 13,55$ & $17,49 \% \pm 22,19$ & $31,02 \% \pm 25,45$ \\
\hline
\end{tabular}

Verglich man alle Scores untereinander, so zeigte sich bei 10,77\% Patienten $(n=130)$ eine Übereinstimmung zwischen allen Scores. Für sie wurde durch alle untersuchten Scores ein 10-Jahres-Frakturrisiko unter $20 \%$ angegeben. In den Risikobereichen $20-30 \%$, sowie $>30 \%$ fand sich zwischen allen aufgeführten Scores keine gemeinsame Schnittmenge an Patienten bezüglich der Risikoklassifikation.

Die vorangegangenen Auswertungen zeigten, dass Differenzen zwischen der Anzahl der Patienten in der Risikostratifizierung in den einzelnen Scores bestanden. Durch die Korrelation nach Kendall-tau-b ließ sich quantifizieren ob es dennoch einen Zusammenhang zwischen den 10-Jahres Frakturrisiken der einzelnen Scores gab. Der abgebildeten Tabelle 17 sind die entsprechenden Korrelationskoeffizienten ( $r$ ) zu entnehmen.

Zwischen dem DVO-Score und den FRAX-Scores unter Auslassung der Knochendichte zeigte sich keine signifikante Korrelation $(p=0,01)$. Eine sehr gering positive Korrelation $(|r| \leq 0,2)$ bestand jeweils für den FRAX- und „FRAX+“-Score einer HF mit BMD und dem DVO-Score 
$(r=0,18, p=0,05)$. Für das Ereignis einer MOF mit BMD zeigte sich zwischen dem FRAX- bzw. „FRAX+"-Score und dem DVO-Score eine gering positive Korrelation $(0,2 \leq|r| \geq 0,5)(r=0,25$, $p=0,01)$. Die stärkste Korrelation zeigte der DVO-Score mit dem Score nach C. Glüer $(r=0,33$, $p=0,01)$.

Die insgesamt stärkste hoch signifikante Korrelation zeigte sich zwischen dem Score nach C. Glüer und dem FRAX- Score einer MOF ohne BMD ( $r=0,38, p=0,01)$. Der Score nach C. Glüer wies positive Korrelationen mit allen FRAX- und „FRAX+“-Scores $(p=0,01)$ auf. Eine sehr gering positive Korrelation $(|r| \leq 0,2)$ lag zwischen dem Score nach C. Glüer und den FRAX- und „FRAX+“-Score für das Ereignis einer HF mit BMD vor. Zwischen den übrigen FRAX-Scores und dem Score nach C. Glüer zeigte sich eine gering positive Korrelation $(0,2 \leq|r| \geq 0,5)$. Es ergaben sich stärkere Korrelationskoeffizienten zwischen den FRAX-Scores und dem Score nach C. Glüer unter Auslassung der Knochendichtemessung. Zudem zeigte sich jeweils eine geringfügig stärkere Korrelation für das Ereignis einer MOF als einer HF. Die Einbeziehung zusätzlicher Risikofaktoren in das „FRAX+“-Modell führte im Vergleich zum FRAX-Modell zu keiner signifikant höheren Korrelation mit dem DVO- Score bzw. Score nach C. Glüer.

Untersucht man die Scores mittels Wilcoxon-Tests, so zeigte sich bei allen Konstellationen ein signifikanter Unterschied $(p=0,01)$ in der zentralen Aussagetendenz.

Tabelle 17: Korrelationskoeffizienten der Fraktur-Scores ( ${ }^{* *}$ Signifikanzniveau 0,01, * Signifikanzniveau 0,05)

\begin{tabular}{|c|c|c|c|c|}
\hline \multicolumn{3}{|c|}{ Korrelationskoeffizienten (r) } & DVO-Score & Score nach C. Glüer \\
\hline \multirow{4}{*}{ FRAX-Score } & \multirow[t]{2}{*}{ ohne BMD } & MOF & 0,061 & $0,38^{\star *}$ \\
\hline & & HF & - & $0,36^{\star \star}$ \\
\hline & \multirow[t]{2}{*}{ mit BMD } & MOF & $0,25^{\star *}$ & $0,22^{* *}$ \\
\hline & & HF & $0,18^{*}$ & $0,18^{\star *}$ \\
\hline \multirow{4}{*}{ „FRAX+“-Score } & \multirow[t]{2}{*}{ ohne BMD } & MOF & $-0,1$ & $0,35^{\star *}$ \\
\hline & & HF & 0,06 & $0,34^{\star *}$ \\
\hline & \multirow[t]{2}{*}{ mit BMD } & MOF & $0,25^{\star *}$ & $0,22^{* *}$ \\
\hline & & HF & $0,18^{*}$ & $0,18^{* *}$ \\
\hline \multicolumn{3}{|c|}{ Score nach C. Glüer } & $0,33^{* *}$ & 1 \\
\hline
\end{tabular}

\subsubsection{Vergleichende Betrachtung der Therapieindikationen zwischen dem DVO-Score aus den Jahren 2006-2014, des Scores nach C. Glüer und der FRAX-Scores}

Basierend auf den in den vorherigen Punkten angeführten Therapiegrenzen konnte für jeden der Scores eine Aussage bezüglich der Therapieindikation getroffen werden. Die 
nachfolgende Abbildung 21 zeigt eine Gegenüberstellung aller Scores bezüglich der beiden Optionen „Therapieindikation ja“ (grün) und „Therapieindikation nein“ (rot).

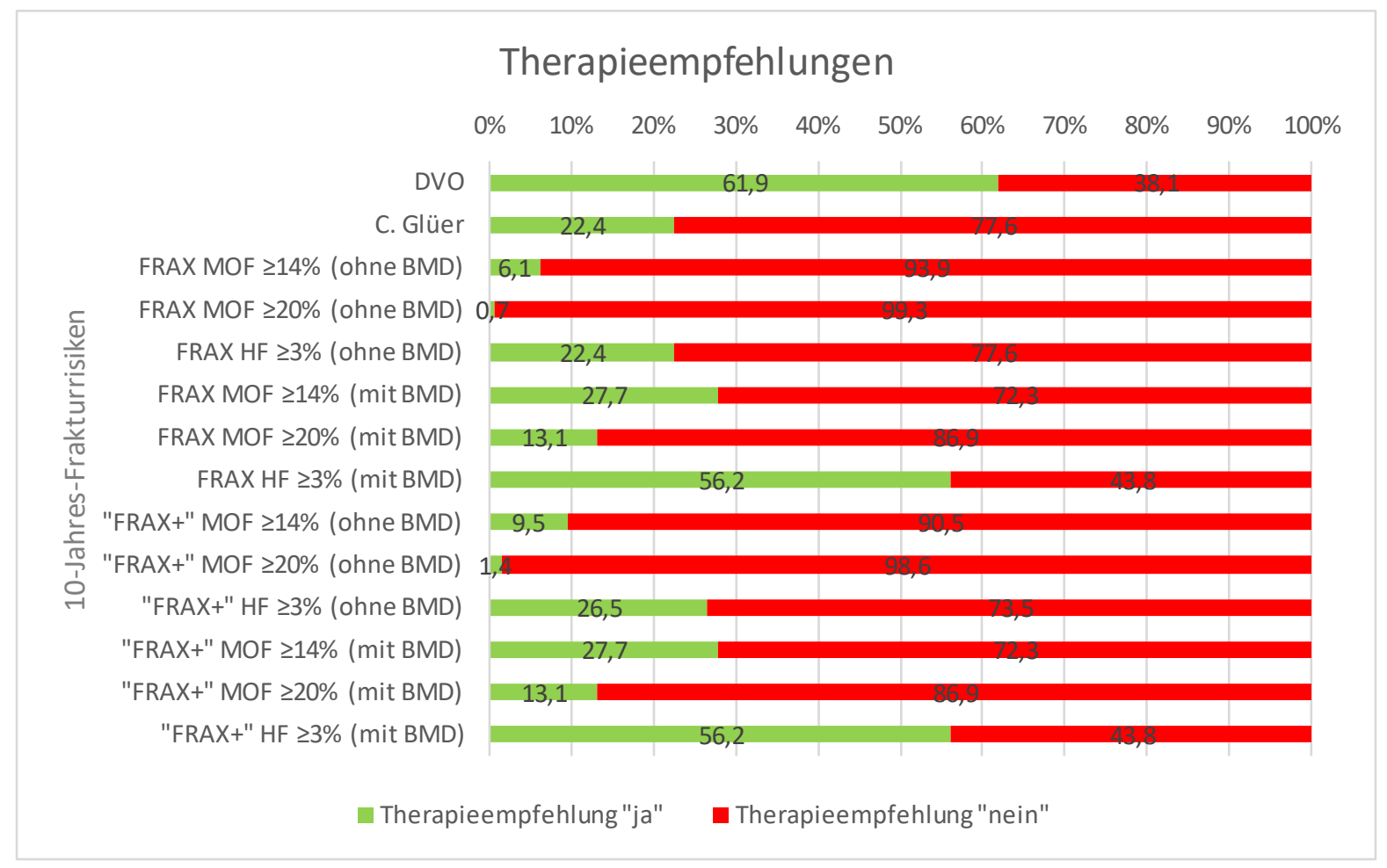

Abbildung 21: Gegenüberstellung der Therapieempfehlungen

Die meisten Therapieempfehlungen ergaben sich basierend auf dem DVO-Score. Es wurde $61,9 \%$ der Patienten eine Therapie empfohlen. Darauf folgten der „FRAX+“-Score, sowie der FRAX-Score für das Ereignis einer HF mit BMD mit jeweils 56,2\% an positiven Therapieempfehlungen. Den wenigsten Patienten $(0,7 \%)$ wurde eine Therapie empfohlen, wenn man den FRAX-Score für das 10-Jahres-Frakturrisiko einer MOF ohne BMD mit einer Therapiegrenze $\geq 20 \%$ zugrunde legte. Im direkten Vergleich zeigte sich, dass die beiden FRAX-Modelle für die jeweiligen Teilscores entweder gleich viele positive Therapieempfehlungen aussprechen (jeweils unter Einbeziehung der Knochendichtemessung) oder basierend auf dem „FRAX+“Score (unter Einbeziehung zusätzlicher Risikofaktoren) mehr Therapieentscheidungen getroffen wurden (ohne Einbeziehung der Knochendichtemessung).

Bei genauerer Analyse zeigte sich, dass allen Patienten, denen basierend auf einem FRAXbzW. „FRAX+“-Score für das 10-Jahres-Risiko einer MOF (ohne und mit BMD) eine Therapie empfohlen wurde, ebenfalls eine Therapie unter Verwendung des FRAX- bzw. „FRAX+“Scores für eine HF (ohne und mit BMD) empfohlen wurde. Diese Aussagen war unabhängig von der gewählten Therapiegrenze von $\geq 14 \%$ bzw. $\geq 20 \%$ zutreffend. Es gab keinen Patienten in dem untersuchten Kollektiv, der eine positive Therapieempfehlung laut FRAX bzw. „FRAX+“-Score für eine MOF (ohne und mit BMD) erhielt, aber keine laut FRAX bzW. „FRAX+“- 
Score für eine HF (ohne und mit BMD). Auch diese Beobachtung traf sowohl für die Therapiegrenze von $\geq 14 \%$ bzw. $\geq 20 \%$ für eine MOF zu.

Unter Verwendung des Scores nach C. Glüer wurde 22,45\% (33 von 147) der Patienten eine Therapie empfohlen. Diese Anzahl an Therapieempfehlungen stimmte mit der Anzahl der Therapieentscheidungen basierend auf dem FRAX-Modell für das Ereignis einer HF ohne Einbeziehung der Knochendichtemessung überein. Es zeigte sich jedoch, dass nur 13 Patienten der 33 zwischen diesen beiden Scores bezüglich der Therapieempfehlung „ja“ übereinstimmten.

Betrachtete man nur die Patienten, die laut DVO-Score ein 10-Jahres-Frakturrisiko über 30\% hatten (und denen somit zugleich eine positive Therapieempfehlung ausgesprochen wurde) und analysierte die errechneten 10-Jahres-Frakturrisiken basierend auf dem FRAX- bzw. „FRAX+“-Score bzw. Score nach C. Glüer, so zeigt sich das in der Tabelle 18 dargestellte Ergebnis: Trotz der Beschränkung der Auswertung auf alle Patienten mit einem über 30\%igem Frakturrisiko laut DVO-Score zeigten sich für die FRAX-Scores und den Score nach C. Glüer durchschnittliche 10-Jahres-Frakturrisiken zwischen 2,47 (FRAX-Score HF ohne BMD) und 14,43 (FRAX bzw. „FRAX+“ MOF mit BMD). Die höchsten durchschnittlichen Frakturrisiken errechneten sich für den FRAX- bzw. „FRAX+“-Score für das Ereignis einer MOF mit Einbeziehung der Knochendichtemessung. Das durchschnittliche Risiko lag bei $14,43 \% \pm$ 12,99. 6,32\% der Patienten hatten ein 10-Jahres-Risiko über 30\%. Für die gleichen Patienten errechnete sich auch ein über 30\%iges 10-Jahres-Frakturrisiko basierend auf dem FRAXbzw. „FRAX+“-Score für eine HF mit BMD. Das durchschnittliche Risiko lag hier bei 9,18\% \pm 12,85. Für den Score nach C. Glüer errechnete sich für diejenigen Patienten, die laut DVOScore ein über 30\%iges 10-Jahres-Frakturrisiko haben ein durchschnittliches Risiko von 3,1\% $\pm 2,56$. Es zeigte sich bei $37,36 \%$ der Patienten ein 10 -Jahres-Frakturrisiko über $30 \%$.

Diese Gegenüberstellung zeigte, dass es starke Abweichungnen der ermittelten 10-JahresFrakturrisiken zwischen den einzelnen Scores gab. 
Tabelle 18: Gegenüberstellung der Patienten mit DVO-Score $>30 \%$ unter Angabe der durchschnittlichen 10-JahresFrakturrisiken in den FRAX-Scores und dem Score nach C. Glüer

\begin{tabular}{|c|c|c|c|c|c|c|c|c|c|c|}
\hline \multicolumn{3}{|c|}{ Scores } & $n$ & $\begin{array}{l}\text { Therapiee } \\
\text { mpfehlung } \\
\text {,ja“ laut } \\
\text { DVO-Score } \\
(<30 \% \quad 10- \\
\text { jahres- } \\
\text { Frakturrisik } \\
\text { o) } \\
\end{array}$ & $\begin{array}{l}\text { Mittel } \\
\text { wert }\end{array}$ & $\begin{array}{l}\text { Stand } \\
\text { ardab } \\
\text { weich } \\
\text { ung }\end{array}$ & $\begin{array}{l}\text { Minim } \\
\text { um }\end{array}$ & $\begin{array}{l}\text { Maxi } \\
\text { mum }\end{array}$ & $\begin{array}{l}\text { Media } \\
\mathrm{n}\end{array}$ & $\begin{array}{l}\text { Anzahl der Pati- } \\
\text { enten mit einem } \\
\text { 10-Jahres-Frak- } \\
\text { turrisiko > } 30 \%\end{array}$ \\
\hline \multirow{4}{*}{ 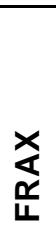 } & \multirow{2}{*}{$\begin{array}{l}\text { Ohne } \\
\text { BMD }\end{array}$} & MOF & 91 & $100 \%$ & 6,92 & 3,81 & 2,00 & 20,0 & 6 & 0 \\
\hline & & HF & 91 & $100 \%$ & 2,47 & 2,8 & 0,1 & 15,0 & 1,6 & 0 \\
\hline & \multirow{2}{*}{$\begin{array}{l}\text { Mit } \\
\text { BMD }\end{array}$} & MOF & 79 & $100 \%$ & 14,43 & 12,99 & 2,20 & 85,0 & 11 & $5(6,32 \%)$ \\
\hline & & $\mathrm{HF}$ & 79 & $100 \%$ & 9,18 & 12,85 & 0,1 & 84,0 & 5,7 & $5(6,32 \%)$ \\
\hline \multirow{4}{*}{ 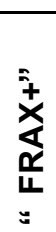 } & \multirow{2}{*}{$\begin{array}{l}\text { Ohne } \\
\text { BMD }\end{array}$} & MOF & 91 & $100 \%$ & 7,36 & 4,44 & 1,3 & 28,0 & 6,2 & 0 \\
\hline & & $\mathrm{HF}$ & 91 & $100 \%$ & 2,78 & 3,28 & 0,1 & 21,0 & 1,5 & 0 \\
\hline & \multirow{2}{*}{\begin{tabular}{|l} 
Mit \\
BMD
\end{tabular}} & MOF & 79 & $100 \%$ & 14,43 & 12,99 & 2,2 & 85,0 & 11 & $5(6,32 \%)$ \\
\hline & & $\mathrm{HF}$ & 79 & $100 \%$ & 9,18 & 12,85 & 0,1 & 84,0 & 5,7 & $5(6,32 \%)$ \\
\hline \multicolumn{3}{|c|}{ C. Glüer } & 91 & $100 \%$ & 3,1 & 2,56 & 0,3 & 10,01 & 2,99 & $34(37,36 \%)$ \\
\hline
\end{tabular}

Bezüglich der Therapieempfehlungen zeigte sich, dass nicht alle Patienten, die durch den DVO-Score als therapiebedürftig eingestuft wurden, ebenfalls durch die FRAX-Scores oder den Score nach C. Glüer als solche erkannt wurden. Die Abbildung 22 veranschaulicht dieses Ergebnis. Die grün hinterlegten Balken stellen den prozentualen Anteil (am Gesamtkollektiv) der Übereinstimmungen der patientenspezifischen Therapieempfehlungen ,ja“ zwischen dem DVO-Score und dem Score nach C. Glüer bzw. den FRAX-Scores dar. Grau hinterlegt ist der Anteil an Patienten, die zusätzlich zu den Patienten, für die eine Übereinstimmung vorlag, durch den Score nach C. Glüer bzw. die FRAX-Scores als therapiebedürftig eingestuft wurden. Blau hinterlegt ist der prozentuale Anteil am Gesamtkollektiv, der im Vergleich mit den sich überschneidenden Therapieempfehlungen „ja“ zusätzlich durch den DVO-Score als therapiebedürftig eingestuft wurden.

Es zeigte sich, dass der höchste Anteil an prozentualen Therapieübereinstimmungen zwischen dem DVO-Score und dem FRAX- bzw. „FRAX+“-Score für eine HF (mit BMD) bestand. Sie zeigten eine Übereinstimmung mit dem DVO-Socre von 40,8\%. Die niedrigste patientenbezogene prozentuale Übereinstimmung lag zwischen dem DVO-Sore und dem FRAX- bzw. „FRAX+“-Score für eine MOF bei einerTherapieschwelle $\geq 20 \%$ vor. Nur bei $0,7 \%$ der Patienten des Gesamtkollektivs zeigte sich eine Übereinstimung der Therapieempfehlung „ja“ zwischen den gennanten FRAX-Scores und dem DVO-Score. Ebenfalls zeigte sich, dass nicht alle Patienten, die eine positive Therapieemfehlung basierend auf dem Score nach CGlüer bzw. den FRAX-Scores auch eine positive Therapieempfehlung basierend auf dem DVO-Score erhielten. Die prozentualen Anteile dieser Patienten am Gesamtkollektiv sind in der Abbildung 22 grau hinterlegt. Für den FRAX- bzw „FRAX+“-Score für das Ereignis einer 
HF (mit BMD) zeigte sich, dass 15,4\% (20 von 130 Patienten) der Patienten des Gesamtkollektivs eine Therapie empfohlen wurde, die laut DVO-Score nicht als therapiebedürttig eingestuft wurden. Für die weiteren FRAX- bzw. „FRAX+“-Scores schwankte der Anteil derjenigen Patienten, die zwar durch die FRAX- bzW. „FRAX+“-Score jedoch nicht durch den DVO-Score als therapiebedürftig eingeordnet wurden zwischen 0,0\% (FRAX MOF $\geq 20 \%$ (ohne BMD)) und 5,4\% (,FRAX+“ HF $\geq 3 \%$ (ohne BMD)).

In der Auswertung der positiven Therapieempfehlungen für den Score nach C. Glüer fand sich bei $19 \%$ der Patienten eine Übereinstimmung mit dem DVO-Score. 3,4\% der männlichen Patienten erhielten eine positive Therapieempfehlung basierend auf dem Score nach C. Glüer, jedoch nicht durch den DVO-Score. 42,9\% der Patienten erhielten wiederum eine Therapieemfehlung durch den DVO-Score, die nicht durch den Score nach C. Glüer als therapiebedürftig eingestuft wurden.

Einer Großzahl von Patienten, die weder durch den Score nach C. Glüer, noch durch den FRAX- bzw. „FRAX+“-Score als therapiebedürftig eingetuft wurden, erhielten basierend auf dem DVO-Score eine positive Therapieempfehlung. Im Vergleich mit den anderen Scores zeigte sich, dass zusätzlich zwischen 20\% (im Vergleich zum FRAX- bzw. „FRAX+“-Scores für eine $\mathrm{HF}$ (mit BMD)) und 61,2\% (im Verlgeich zum FRAX-Score MOF $\geq 20 \%$ (ohne BMD)) der Patienten eine Therapie anhand des DVO-Scores empfohen wurde. 


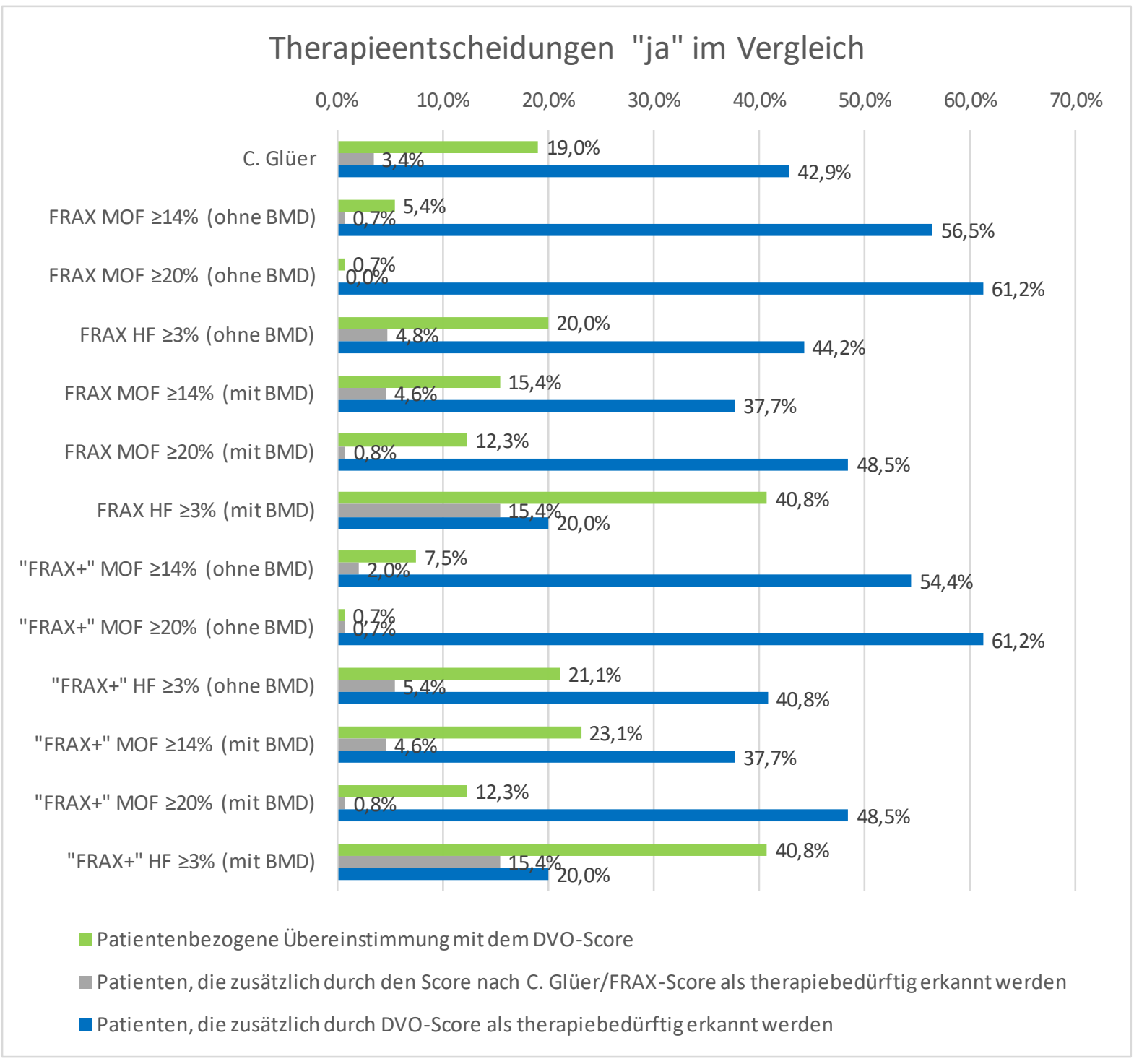

Abbildung 22: Patientenbezogene Therapieübereinstimmungen bzw. Unterschiede zwischen den einzelnen $10-$ Jahres-Frakturrisiko-Scores anteilig am Gesamtkollektiv

Untersuchte man alle Scores mittels McNemar-Tests hinsichtlich der Gemeinsamkeiten und Unterschiede in den Therapieentscheidungen „ja“ und „nein“, so zeigte sich das in der Tabelle 19 dargestellte Ergebnis. Es gab keinen signifikanten Unterscheid in den Therapieentscheidungen zwischen dem DVO-Score und den FRAX-, sowie „FRAX+“-Scores für das Ereignis einer HF unter Einbeziehung der Knochendichtemessung. Alle anderen FRAX- und „FRAX+“Teilscores unterschieden sich signifikant in der Gesamtheit der Therapieempfehlungen vom DVO-Score.

Für den Score nach C. Glüer zeigte sich kein signifikanter Unterschied in Bezug auf die Therapieempfehlungen, die basierend auf dem FRAX- und „FRAX+“-Score für das 10-Jahresrisiko einer HF ohne Knochendichtemessung getroffen wurden. Ebenso zeigte sich kein signifikanter Unterschied in Bezug auf die FRAX- und „FRAX+“-Scores für eine MOF (unabhängig von einer gewählten Therapieschwelle) unter Einbeziehung der Knochendichtemessung. 
Zwischen den FRAX- und „FRAX+“-Scores zeigte sich jeweils für die entsprechenden Scores mit der gleichen Aussageabsicht kein signifikanter Unterscheid in den Therapieentscheidungen. Unter Einbeziehung der Knochendichtemessung zeigte sich sowohl für eine MOF (unabhängig von der gewählten Therapieschwelle) als auch für eine HF eine Übereinstimmung von $100 \%$ zwischen dem FRAX- und „FRAX+“-Score.

Tabelle 19: McNemar-Test $(p=0,01)$

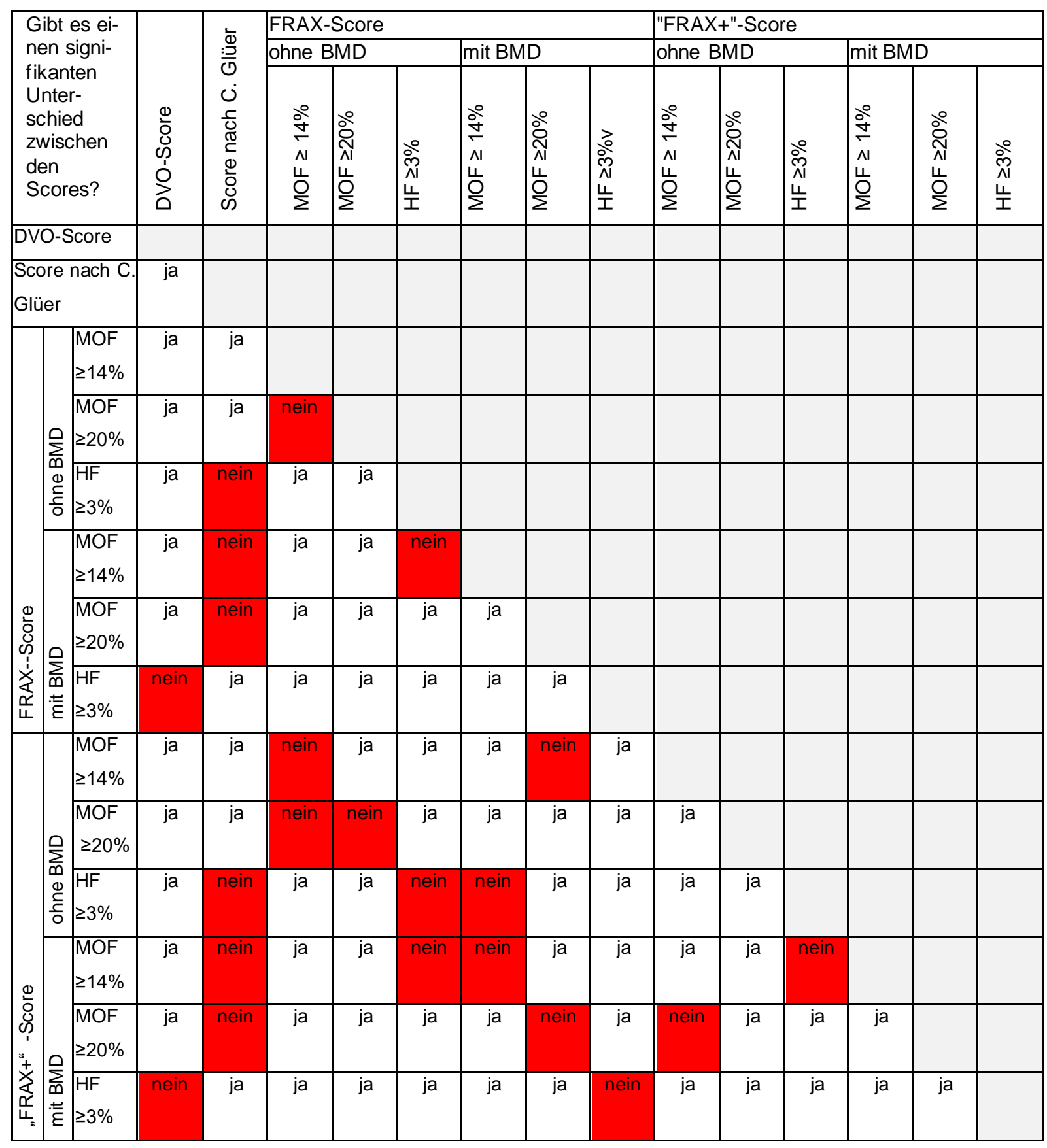

Um genauer zu analysieren, wie hoch die prozentuale Übereinstimmung der Therapieentscheidungen pro Patient zwischen den einzelnen Scores war, erfolgte eine direkte Gegenüberstellung. Diese findet sich in der Tabelle 20. Die Übereinstimmungen der Therapieentscheidungen bezogen sich dabei sowohl auf die Therapieentscheidung „ja“, als auch „nein“. 
Die Angabe erfolgte in Prozent der Gesamtanzahl der verglichenen Patienten. Zusätzlich wurde cohens kappa ( $\mathrm{k})$ als Maß der Interraterreliabilität angegeben.

Der DVO-Score zeigte den höchsten Konsens an Therapieentscheidungen mit dem „FRAX+“Score für das 10-Jahresrisiko einer HF mit BMD. Sie stimmten bei $65,6 \%$ der Patienten überein. Es zeigte sich ein schwacher Grad der Übereinstimmung ( $k=0,273, p=0,01)$. Die geringste Schnittmenge an Therapieübereinstimmungen $(38,8 \%)$ zeigte der DVO-Score mit dem FRAXScore für eine MOF mit einer festgelegten Therapiegrenze von $\geq 20 \%$ ohne BMD. Ebendieser FRAX-Score zeigte jedoch den höchsten Prozentsatz an übereinkommenden Therapieentscheidungen $(78,2 \%)$ mit dem Score nach C. Glüer. Die wenigsten Übereinstimmungen lagen für den Score nach C. Glüer mit dem FRAX- bzw. „FRAX+“-Score einer HF mit BMD vor. Sie zeigten bei $55,4 \%$ der Patienten eine Therapieübereinstimmung ( $k=0,165, p=0,05)$.

Die insgesamt höchste Übereinstimmung bezüglich der Therapieoptionen „ja“ bzw. „nein“ fand sich zwischen dem FRAX- und dem „FRAX-+“-Score für eine MOF unabhängig von der gewählten Therapiegrenze sowie einer HF, jeweils unter Einbeziehung der Knochendichtemessung. Diese beiden Scores zeigten eine Übereinstimmung von $100 \%(\mathrm{k}=1)$. Auch ohne Berücksichtigung der Knochendichtemessung zeigte sich zwischen den FRAX- und „FRAX+“Modellen ein hoher Prozentsatz identischer Therapieentscheidung von 96,6\% für eine MOF mit der Therapiegrenze $\geq 14 \%$ ( $\kappa=0,765$ ), 99,32\% für eine MOF mit einer Therapiegrenze $\geq 20 \%(\kappa=0,664)$ bzw. 95,24\% für eine HF $(\kappa=0,856)$.

Analysierte man alle Scores bezüglich der Übereinstimmung der Therapieoption „ja“, so zeigte sich, dass es nur einen Patienten gab, bei dem alle Scores in ihrer Entscheidung übereinstimmen. In der Therapieoption „nein“ zeigten sich 26 Übereinstimmungen zwischen allen Scores. 
Tabelle 20: Übereinstimmung der Therapieentscheidungen in \% ( ${ }^{* *}$ Signifikanzniveau 1\%, *Signifikanzniveau 5\%)

\begin{tabular}{|c|c|c|c|c|c|c|c|c|c|c|c|c|c|c|c|c|}
\hline \multirow{2}{*}{\multicolumn{3}{|c|}{$\begin{array}{l}\text { Übereinstimmung der Therapie- } \\
\text { entscheidungen in \%/ cohens } \\
\text { kappa (к) }\end{array}$}} & \multirow{3}{*}{ 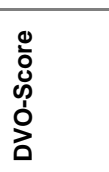 } & \multirow{3}{*}{ 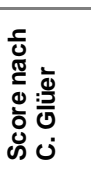 } & \multicolumn{6}{|c|}{ FRAX-Score } & \multicolumn{6}{|c|}{ "FRAX+"-Score } \\
\hline & & & & & \multicolumn{3}{|c|}{ ohne BMD } & \multicolumn{3}{|c|}{ mit BMD } & \multicolumn{3}{|c|}{ ohne BMD } & \multicolumn{3}{|c|}{ mit BMD } \\
\hline & & & & & $\begin{array}{l}\text { MOF } \\
\geq 14 \%\end{array}$ & $\begin{array}{l}\text { MOF } \\
\geq 20 \%\end{array}$ & $\begin{array}{l}\text { HF } \\
\geq 3 \%\end{array}$ & $\begin{array}{l}\text { MOF } \\
\geq 14 \%\end{array}$ & $\begin{array}{l}\text { MOF } \\
\geq 20 \%\end{array}$ & $\begin{array}{l}\mathrm{HF} \\
\geq 3 \%\end{array}$ & $\begin{array}{l}\text { MOF } \\
\geq 14 \%\end{array}$ & $\begin{array}{l}\text { MOF } \\
\geq 20 \%\end{array}$ & $\begin{array}{l}\mathrm{HF} \\
\geq 3 \%\end{array}$ & $\begin{array}{l}\text { MOF } \\
\geq 14 \%\end{array}$ & $\begin{array}{l}\text { MOF } \\
\geq 20 \%\end{array}$ & $\begin{array}{l}\text { HF } \\
\geq 3 \%\end{array}$ \\
\hline \multicolumn{17}{|c|}{ DVO-Score } \\
\hline \multicolumn{3}{|c|}{ Score nach C. Glüer } & $\begin{array}{l}53,74 / \\
0,182^{\star \star}\end{array}$ & & & & & & & & & & & & & \\
\hline \multirow{6}{*}{ 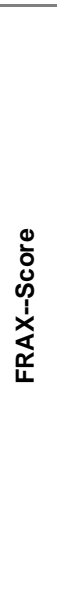 } & \multirow{3}{*}{ 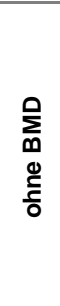 } & MOF $\geq 14 \%$ & $\begin{array}{l}42,86 / \\
0,055^{\star}\end{array}$ & $\begin{array}{l}76,86 / \\
0,104\end{array}$ & & & & & & & & & & & & \\
\hline & & MOF $\geq 20 \%$ & $\begin{array}{l}38,8 / \\
0,008\end{array}$ & $\begin{array}{l}78,2 / \\
0,046\end{array}$ & $\begin{array}{l}94,56 / \\
0,19^{* *}\end{array}$ & & & & & & & & & & & \\
\hline & & $\mathrm{HF} \geq 3 \%$ & $\begin{array}{l}51,7 / \\
0,144^{*}\end{array}$ & $\begin{array}{l}72,1 / \\
0,27^{*}\end{array}$ & $\begin{array}{l}83,67 / \\
0,368^{* *}\end{array}$ & $\begin{array}{l}77,56 / \\
0,045\end{array}$ & & & & & & & & & & \\
\hline & \multirow{3}{*}{$\begin{array}{l}\sum_{m}^{0} \\
\frac{\bar{E}}{\mathrm{E}}\end{array}$} & MOF $\geq 14 \%$ & $\begin{array}{l}57,69 / \\
0,228^{\star \star}\end{array}$ & $\begin{array}{l}70 / \\
0,203^{*}\end{array}$ & $\begin{array}{l}76,15 / \\
0,208^{\star \star}\end{array}$ & $\begin{array}{l}73,08 / \\
0,04\end{array}$ & $\begin{array}{l}75,38 / \\
0,338^{\star *}\end{array}$ & & & & & & & & & \\
\hline & & MOF $\geq 20 \%$ & $\begin{array}{l}50,8 / \\
0,151^{\star *}\end{array}$ & $\begin{array}{l}73,8 / \\
0,115\end{array}$ & $\begin{array}{l}90,77 / \\
0,459^{* *}\end{array}$ & $\begin{array}{l}87,7 / \\
0,098^{*}\end{array}$ & $\begin{array}{l}80,77 / \\
0,385^{\star *}\end{array}$ & $\begin{array}{l}85,38 / \\
0,564^{\star *}\end{array}$ & & & & & & & & \\
\hline & & $\mathrm{HF} \geq 3 \%$ & $\begin{array}{l}64,6 / \\
0,273^{\star \star}\end{array}$ & $\begin{array}{l}55,4 / \\
0,165^{\star}\end{array}$ & $\begin{array}{l}49,23 / \\
0,085^{\star}\end{array}$ & $\begin{array}{l}44,6 / \\
0,012\end{array}$ & $\begin{array}{l}57,7 / \\
0,204^{\star \star}\end{array}$ & $\begin{array}{l}71,54 / \\
0,46^{\star *}\end{array}$ & $\begin{array}{l}56,9 / \\
0,21^{\star *}\end{array}$ & & & & & & & \\
\hline \multirow{6}{*}{ 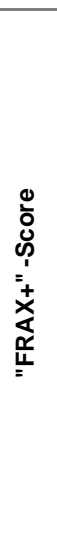 } & \multirow{3}{*}{ 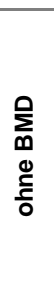 } & MOF $\geq 14 \%$ & $\begin{array}{l}43,54 / \\
0,053\end{array}$ & $\begin{array}{l}74,82 / \\
0,091\end{array}$ & $\begin{array}{l}96,6 / \\
0,765^{\star \star}\end{array}$ & $\begin{array}{l}91,16 / \\
0,122^{\star *}\end{array}$ & $\begin{array}{l}84,35 / \\
0,435^{\star \star}\end{array}$ & $\begin{array}{l}78,46 / \\
0,323^{\star *}\end{array}$ & $\begin{array}{l}90 / \\
0,497^{* *}\end{array}$ & $\begin{array}{l}71,54 / \\
0,46^{* *}\end{array}$ & & & & & & \\
\hline & & MOF $\geq 20 \%$ & $\begin{array}{l}44,9 / \\
-0,005\end{array}$ & $\begin{array}{l}77,6 / \\
0,032\end{array}$ & $\begin{array}{l}95,24 / \\
0,349^{\star \star}\end{array}$ & $\begin{array}{l}99,32 / \\
0.664^{\star *}\end{array}$ & $\begin{array}{l}78,23 / \\
0,088^{\star *}\end{array}$ & $\begin{array}{l}73,85 / \\
0,078^{*}\end{array}$ & $\begin{array}{l}88,46 / \\
0,188^{* *}\end{array}$ & $\begin{array}{l}45,38 / \\
0,024\end{array}$ & $\begin{array}{l}91,84 / \\
0,232^{\star \star}\end{array}$ & & & & & \\
\hline & & $\mathrm{HF} \geq 3 \%$ & $\begin{array}{l}53,71 / \\
0,168\end{array}$ & $\begin{array}{l}70,01 / \\
0,193^{\star}\end{array}$ & $\begin{array}{l}79,59 / \\
0,306^{\star \star}\end{array}$ & $\begin{array}{l}74,15 / \\
0,037\end{array}$ & $\begin{array}{l}95,24 / \\
0,873^{\star \star}\end{array}$ & $\begin{array}{l}73,07 / \\
0,333^{\star \star}\end{array}$ & $\begin{array}{l}78,46 / \\
0,368^{* *}\end{array}$ & $\begin{array}{l}63,08 / \\
0,299^{\star \star}\end{array}$ & $\begin{array}{l}82,99 / \\
0,451^{\star *}\end{array}$ & $\begin{array}{l}74,83 / \\
0,074^{\star}\end{array}$ & & & & \\
\hline & \multirow{3}{*}{ 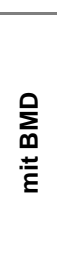 } & MOF $\geq 14 \%$ & $\begin{array}{l}57,69 / \\
0,228^{\star *}\end{array}$ & $\begin{array}{l}70 / \\
0,203^{\star}\end{array}$ & $\begin{array}{l}76,15 / \\
0,208^{\star \star}\end{array}$ & $\begin{array}{l}73,08 / \\
0,04\end{array}$ & $\begin{array}{l}74,62 / \\
0,338^{\star \star}\end{array}$ & $\begin{array}{l}100 / \\
1^{\star \star}\end{array}$ & $\begin{array}{l}85,38 / \\
0,564^{\star \star}\end{array}$ & $\begin{array}{l}71,54 / \\
0,46^{\star \star}\end{array}$ & $\begin{array}{l}78,46 / \\
0,323^{\star *}\end{array}$ & $\begin{array}{l}73,85 / \\
0,078^{*}\end{array}$ & & & & \\
\hline & & MOF $\geq 20 \%$ & $\begin{array}{l}50,8 / \\
0,151^{\star \star}\end{array}$ & $\begin{array}{l}73,8 / \\
0,115\end{array}$ & $\begin{array}{l}90,77 / \\
0,459^{\star *}\end{array}$ & $\begin{array}{l}87,7 / \\
0,098^{\star}\end{array}$ & $\begin{array}{l}80,77 / \\
0,385^{\star \star}\end{array}$ & $\begin{array}{l}85,38 / \\
0,564^{\star *}\end{array}$ & $\begin{array}{l}100 / \\
1^{\star *}\end{array}$ & $\begin{array}{l}56,92 / \\
0,21^{* *}\end{array}$ & $\begin{array}{l}90 / \\
0,497^{\star \star}\end{array}$ & $\begin{array}{l}88,5 / \\
0,188^{* *}\end{array}$ & $\begin{array}{l}78,5 / \\
0,368^{* *}\end{array}$ & & & \\
\hline & & $\mathrm{HF} \geq 3 \%$ & $\begin{array}{l}64,6 / \\
0,273^{* *}\end{array}$ & $\begin{array}{l}55,4 / \\
0,165^{\star}\end{array}$ & $\begin{array}{l}49,23 / \\
0,085^{\star}\end{array}$ & $\begin{array}{l}44,6 / \\
0,012\end{array}$ & $\begin{array}{l}57,7 / \\
0,204^{* *}\end{array}$ & $\begin{array}{l}56,15 / \\
0,46^{\star *}\end{array}$ & $\begin{array}{l}56,9 / \\
0,21^{\star *}\end{array}$ & $\begin{array}{l}100 / \\
1^{* *}\end{array}$ & $\begin{array}{l}51,54 / \\
0,119^{* *}\end{array}$ & $\begin{array}{l}45,38 / \\
0,024\end{array}$ & $\begin{array}{l}63,08 / \\
0,299^{\star \star}\end{array}$ & & $\begin{array}{l}56,92 / \\
0,21^{\star *}\end{array}$ & \\
\hline
\end{tabular}




\subsection{Vergleichende Betrachtung der Therapieempfehlungen basierend auf den WHO-Kriterien und dem DVO-Score, FRAX-Score, „FRAX+“-Score bzw. Score nach C. Glüer}

Durch die WHO wurde 1991 ein T-Score, gemessen an der Lendenwirbelsäule, dem Gesamtfemur oder dem proximalen Femurhals, von $\leq-2,5$ Standardabweichung vom statistischen Mittelwert gesunder 20- bis 29jährigen Frauen zur Diagnose einer Osteoporose festgelegt (WHO 1991, 2003). Basierend auf diesem Kriterium lag bei 63,84\% (83 von 130 Patienten) des untersuchten Kollektivs männlicher Patienten eine therapiebedürftige Osteoporose vor. Im Vergleich mit den untersuchten Scores basierend auf den DVO-Leitlinien, dem FRAX-Score und Score nach C. Glüer, zeigt sich somit ein höherer Anteil an prozentualen Therapieentscheidungen (vergleiche Abbildung 21). Vergleicht man die Scores bezüglich ihrer Übereinstimmungen der Therapieentscheidungen „ja“ und „nein“, so unterscheidet sich die WHO-basierte Therapieempfehlung signifikant von allen anderen Scores außer vom DVO-Score und den FRAXScores für eine Hüftfraktur unter Einbeziehung der Knochendichtemessung. Die WHO-basierten Therapieempfehlungen zeigten eine prozentuale, patientenbezogene Übereinstimmung mit dem DVO-Score von 70,77\% (,ja und nein“) bzw. 47,69\% (,ja“). Der FRAX-Score für eine HF unter Einbeziehung der Knochendichtemessung zeigte eine prozentuale Übereinstimmung mit den WHO-basierten Therapieempfehlungen von 69,23\% (,ja und nein“) bzw. 44,62 in Bezug auf Therapie „ja“.

\subsection{Multiple lineare Regression}

Ziel der linearen Regressionsanalyse ist es, eine abhängige Variable (in diesem Fall das FRAX- und „FRAX+“-10-Jahres-Frakturrisiko) durch eine oder mehrere unabhängige Variablen (in diesem Fall die in den FRAX- bzw. „FRAX+“-Score einfließenden Variablen) zu erklären bzw. zu prognostizieren. Dabei ist es möglich, sowohl die Stärke des Einflusses, jeder einzelnen unabhängigen Variablen auf die abhängige Variable zu bestimmen, als auch die Veränderung der abhängigen Variablen bei einer Variation der unabhängigen Variablen. Diese Aussagen lassen sich aus dem Regressionskoeffizienten ablesen.

\subsubsection{Lineares Regressionsmodell für das FRAX-10-Jahres-Frakturrisiko einer „major osteoporotic“ Fraktur ohne und mit Einbeziehung der Knochendichtemessung}

Durch das lineare Regressionsmodell ließ sich der Einfluss der unabhängigen Variablen am 10-Jahres-Frakturrisiko für eine MOF berechnen. Der Determinationskoeffizient $r^{2}$ beschreibt den Anteil der erklärten Varianz an der Gesamtvarianz des Modells. Im FRAX-Modell für das 10-Jahresrisiko einer MOF ohne Einbeziehung der Knochendichtemessung lag $r^{2}$ bei $88 \%$, mit Einbeziehung der Knochendichtemessung bei 74\%. Für keines der beiden Modelle zeigte sich eine Multikollinearität zwischen den unterschiedlichen Variablen. Für keines der beiden 
Modelle zeigte sich eine starke Korrelation zwischen den unterschiedlichen Variablen (somit keine Multikollinearität), sodass die Schätzung der Regression verwendet werden konnte.

Betrachtete man das lineare Regressionsmodell für eine MOF ohne Einbeziehung der Knochendichtemessung, so hatten alle berücksichtigen Faktoren außer "gegenwärtiges Rauchen“ und „Rheumatoide Arthritis“ auf einem Signifikanzniveau von einem Prozent einen hoch signifikanten Einfluss auf das Modell. Der stärkste signifikante Koeffizient ergab sich für die Variable „Hüftfraktur eines Elternteils“. Lag demnach eine Hüftfraktur eines Elternteils vor, so stieg das Frakturrisiko um 4,32 Prozentpunkte.

Unter Einbeziehung der Knochendichtemessung zeigte sich für fünf der zehn untersuchten Variablen ein hoch signifikanter Einfluss $(p=0,01)$ an der erklärten Varianz des Modells. Keinen signifikanten Einfluss hatten die Faktoren: „Alter“, „BMI“, „gegenwärtiges Rauchen“, „Rheumatoide Arthritis“, sowie „sekundäre Osteoporose“. Auch hier errechnete sich für den Faktor „Hüftfraktur eines Elternteils“ der höchste signifikante Koeffizient. Lag dieser Risikofaktor vor, so stieg das 10-Jahres-Risiko einer MOF mit BMD um 8,92 Prozentpunkte.

Tabelle 21: Regressionsmodell FRAX-Score MOF ohne $\left(r^{2}=0,88\right)$ bzw. mit $\left(r^{2}=0,74\right)$ BMD ( ${ }^{* *}$ signifikant auf einem Signifikanzniveau von 1\%, * signifikant auf einem Signifikanzniveau von 5\%)

\begin{tabular}{|c|c|c|c|c|c|c|}
\hline \multirow[t]{2}{*}{ Variablen } & \multicolumn{3}{|c|}{ FRAX-Score für eine MOF ohne BMD } & \multicolumn{3}{|c|}{ FRAX-Score für eine MOF mit BMD } \\
\hline & $\begin{array}{l}\text { Regressionskoef- } \\
\text { fizient }\end{array}$ & $\begin{array}{l}\text { Robuster } \\
\text { Standard- } \\
\text { fehler }\end{array}$ & $\begin{array}{l}\text { p- } \\
\text { Wert }\end{array}$ & $\begin{array}{l}\text { Regressionskoef- } \\
\text { fizient }\end{array}$ & $\begin{array}{l}\text { Robuster } \\
\text { Standard- } \\
\text { fehler }\end{array}$ & $\begin{array}{l}\text { p- } \\
\text { Wert }\end{array}$ \\
\hline Alter & 0,16 & 0,14 & $0,00^{\star \star}$ & $-0,53$ & 0,5 & 0,29 \\
\hline BMI & $-0,14$ & 0,22 & $0,00^{\star \star}$ & $-0,12$ & 0,11 & 0,29 \\
\hline $\begin{array}{l}\text { Vorausgehende } \\
\text { Frakturen }\end{array}$ & 3,53 & 0,27 & $0,00^{\star \star}$ & 5,26 & 0,83 & $0,00^{* \star}$ \\
\hline $\begin{array}{l}\text { Hüftfraktur eines } \\
\text { Elternteils }\end{array}$ & 4,32 & 0,62 & $0,00^{\star \star}$ & 8,92 & 2,64 & $0,00^{* *}$ \\
\hline $\begin{array}{l}\text { Gegenwärtiges } \\
\text { Rauchen }\end{array}$ & 0,28 & 0,27 & 0,30 & 0,86 & 1,09 & 0,94 \\
\hline $\begin{array}{l}\text { Glukokortikoste- } \\
\text { roide }\end{array}$ & 3,12 & 0,34 & $0,00^{\star \star}$ & 7,13 & 1,64 & $0,00^{\star \star}$ \\
\hline $\begin{array}{l}\text { Rheumatoide } \\
\text { Arthritis }\end{array}$ & 1,45 & 0,7 & 0,4 & 4,96 & 2,32 & $0,03^{*}$ \\
\hline $\begin{array}{l}\text { Sekundäre Oste- } \\
\text { oporose }\end{array}$ & 1,07 & 0,28 & $0,00^{\star \star}$ & 2,83 & 1,44 & $0,04^{*}$ \\
\hline $\begin{array}{lll}\text { Alkohol } & 3 & \text { und } \\
\text { mehr } & \text { Einhei- }\end{array}$ & 2,14 & 0,49 & $0,00^{* *}$ & 6,53 & 2,05 & $0,00^{* *}$ \\
\hline T-Score & - & - & - & $-6,64$ & 2,05 & $0,00^{* *}$ \\
\hline Konstante & $-2,97$ & 0,92 & $0,00^{* \star}$ & $-2,2$ & 4,33 & 0,61 \\
\hline
\end{tabular}




\subsubsection{Lineares Regressionsmodell für das FRAX-10-Jahres-Frakturrisiko einer Hüft- fraktur ohne und mit Einbeziehung der Knochendichtemessung}

Für das FRAX-Modell der Vorhersage des 10-Jahresrisikos für eine HF ohne BMD errechnete sich für das lineare Regressionsmodell ein $r^{2}$ von 0,73 . Demnach konnten $73 \%$ der Gesamtstreuung der 10-Jahres-Frakturrisiken durch die folgenden fünf Variablen erklärt werden: „Alter", „BMI“, „vorausgehende Frakturen“, „Glukokortikosteroide“, sowie „Alkohol 3 und mehr Einheiten/Tag“. Sie alle hatten einen hoch signifikanten $(p=0,01)$ Einfluss auf das Modell. Der höchste Regressionskoeffizient errechnete sich für die Variable „Glukokortikosteroide“. Lag dieser Risikofaktor vor, so stieg das 10-Jahresrisiko für eine Hüftfraktur um 1,91 Prozentpunkte. Es zeigte sich keine Multikollinearität zwischen den einzelnen Variablen. Folglich zeigte sich für dieses Modell keine starke Interferenz der einzelnen Faktoren, die mit einer Ungenauigkeit der Schätzung der Regrerssionskoeffizienten einhergegangen wäre.

Das $\mathrm{r}^{2}$ für das FRAX-Modell unter Einbeziehung der Knochendichtemessung lag mit 0,62 niedriger als im vorherigen Modell. Die Variablen „vorausgehende Fraktur“, „Glukokortikosteroide“, „Alkohol 3 und mehr Einheiten/Tag“, sowie der T-Score hatten auf einem Signifikanzniveau von einem Prozent einen signifikanten Einfluss auf das lineare Regressionsmodell. Sie erklärten $62 \%$ der Gesamtstreuung des 10-Jahresrisikos einer Hüftfraktur. Zwischen den einzelnen Faktoren zeigte sich keine Kollinearität. Der höchste Regressionskoeffizient errechnete sich in diesem Modell für den Faktor „Alkohol 3 und mehr Einheiten/Tag“ (Regressionskoeffizient: 6,62), gefolgt vom T-Score. Fiehl der T-Score um eine Einheit ab, so stieg das 10-Jahresrisiko für eine Hüftfraktur um 6,3 Prozentpunkte. Der niedrigste signifikante Regressionskoeffizient errechnete sich mit 2,8 für die Variable „vorausgehende Frakturen“ Eine genaue Auflistung aller Variablen mit den zugehörigen Parametern findet sich in Tabelle 22. 
Tabelle 22: Regressionsmodell FRAX-Score Hüftfraktur ohne $\left(r^{2}=0,73\right)$ bzw. mit $\left(r^{2}=0,62\right) B M D\left({ }^{* *}\right.$ signifikant auf einem Signifikanzniveau von 1\%, * signifikant auf einem Signifikanzniveau von 5\%)

\begin{tabular}{|c|c|c|c|c|c|c|}
\hline \multirow[t]{2}{*}{ Variablen } & \multicolumn{3}{|c|}{ FRAX-Score für eine HF ohne BMD } & \multicolumn{3}{|c|}{ FRAX-Score für eine HF mit BMD } \\
\hline & $\begin{array}{l}\text { Regressi- } \\
\text { onskoeffi- } \\
\text { zient }\end{array}$ & $\begin{array}{l}\text { Robuster } \\
\text { Standard- } \\
\text { fehler }\end{array}$ & p-Wert & $\begin{array}{l}\text { Regressi- } \\
\text { onskoeffi- } \\
\text { zient }\end{array}$ & $\begin{array}{l}\text { Robuster } \\
\text { Standard- } \\
\text { fehler }\end{array}$ & p-Wert \\
\hline Alter & 0,15 & 0,02 & $0,00^{* *}$ & $-0,05$ & 0,58 & 0,36 \\
\hline BMI & $-0,1$ & 0,02 & $0,00^{* *}$ & $-0,18$ & 0,13 & 0,17 \\
\hline $\begin{array}{l}\text { Vorausgehende Frak- } \\
\text { turen }\end{array}$ & 1,05 & 0,31 & $0,00^{* *}$ & 2,8 & 0,94 & $0,00^{* *}$ \\
\hline $\begin{array}{l}\text { Hüftfraktur eines El- } \\
\text { ternteils }\end{array}$ & 0,82 & 0,71 & 0,25 & 5,36 & 3,21 & 0,10 \\
\hline $\begin{array}{l}\text { Gegenwärtiges Rau- } \\
\text { chen }\end{array}$ & 0,35 & 0,31 & 0,26 & 0,5 & 1,27 & 0,70 \\
\hline Glukokortikosteroide & 1,91 & 0,42 & $0,00^{* *}$ & 5,97 & 1,82 & $0,00^{* *}$ \\
\hline $\begin{array}{l}\text { Rheumatoide Arthri- } \\
\text { tis }\end{array}$ & 0,56 & 0,65 & 0,39 & 4,04 & 2,42 & 0,10 \\
\hline $\begin{array}{l}\text { Sekundäre Osteopo- } \\
\text { rose }\end{array}$ & 0,45 & 0,31 & 0,15 & 3,02 & 1,38 & $0,03^{*}$ \\
\hline $\begin{array}{l}\text { Alkohol } 3 \text { und mehr } \\
\text { Einheiten/Tag }\end{array}$ & 1,58 & 0,55 & $0,00^{* *}$ & 6,62 & 2,42 & $0,01^{* \star}$ \\
\hline T-Score & - & - & - & $-6,3$ & 1,03 & $0,00^{* *}$ \\
\hline Konstante & $-5,33$ & 1,03 & $0,00^{* *}$ & $-3,2$ & 4,84 & 0,52 \\
\hline
\end{tabular}

\subsubsection{Lineares Regressionsmodell für das „FRAX+“ 10-Jahres-Frakturrisiko einer MOF ohne und mit BMD}

Ohne Knochendichtemessung errechnete sich in dem linearen Regressionsmodell für das 10Jahresrisiko einer MOF ein $r^{2}$ von 0,86. Dem zufolge ließen sich $86 \%$ der Gesamtvarianz durch das in Tabelle 23 abgebildete Modell beschreiben. Zwischen den einzelnen Variablen zeigte sich im berechneten Modell keine Multikollinearität. Ein hoch signifikanter Einfluss $(p=0,01)$ ergab sich für sechs der neun Variablen. Keinen signifikanten Einfluss in diesem Modell hatten: „gegenwärtiges Rauchen“ und „Rheumatoide Arthritis“. Der höchste Regressionskoeffizient errechnete sich für die Variable „Hüftfraktur eines Elternteils“. Lag dieser Faktor vor, so erhöhte sich das 10-Jahresrisiko für eine MOF um 4,69 Prozentpunkte.

Im linearen Regressionsmodell des „FRAX+“-Scores für das 10-Jahresrisiko einer MOF mit BMD errechnete sich ein $\mathrm{r}^{2}$ von 0,73 . Die folgenden sechs der insgesamt zehn berücksichtigen Variablen hatten einen hoch signifikanten Einfluss $(p=0,01)$ auf das Modell: „vorausgehende Fraktur“; „Hüftfraktur eines Elternteils“, „Glukokortikosteroide“, „Rheumatoide Arthritis“, „Alkohol 3 und mehr Einheiten /Tag“, sowie der T-Score. Zwischen den einzelnen Variablen zeigte sich keine Multikollinearität. 
Der höchste Korrelationskoeffizient errechnete sich für den Faktor „Hüftfraktur eines Elternteils“. Lag dieses Ereignis vor, so erhöhte sich das 10-Jahresrisiko einer MOF mit BMD um 8,55 Prozentpunkte. Der niedrigste signifikante Korrelationskoeffizient ergab sich für die Variable „vorausgehende Fraktur“. Eine genaue Auflistung aller Variablen mit entsprechenden Parametern findet sich in der Tabelle 23.

Tabelle 23: Regressionsmodell für den „FRAX+" - Score für eine MOF ohne $\left(r^{2}=0,86\right)$ bzw. mit $B M D\left(r^{2}=0,73\right){ }^{* *}$ signifikant auf einem Signifikanzniveau von 1\%, * signifikant auf einem Signifikanzniveau von 5\%)

\begin{tabular}{|l|l|l|l|l|l|l|}
\hline \multirow{2}{*}{ Variablen } & \multicolumn{2}{|l}{ „FRAX+“-Score für eine MOF ohne BMD } & \multicolumn{2}{l|}{ „FRAX+“-Score für eine MOF mit BMD } \\
\cline { 2 - 6 } & $\begin{array}{l}\text { Regressionskoef- } \\
\text { fizient }\end{array}$ & $\begin{array}{l}\text { Robuster } \\
\text { Standard- } \\
\text { fehler }\end{array}$ & $\begin{array}{l}\text { p- } \\
\text { Wert }\end{array}$ & $\begin{array}{l}\text { Regressionskoef- } \\
\text { fizient }\end{array}$ & $\begin{array}{l}\text { Robuster } \\
\text { Standard- } \\
\text { fehler }\end{array}$ & p-Wert \\
\hline Alter & 0,19 & 0,02 & $0,00^{* *}$ & $-0,49$ & 0,49 & 0,32 \\
\hline BMI & -0.17 & 0,03 & $0,00^{* *}$ & $-0,11$ & 0,11 & 0,30 \\
\hline $\begin{array}{l}\text { Vorausgehende } \\
\text { Frakturen }\end{array}$ & 3,87 & 0,29 & $0,00^{* *}$ & 5,2 & 0,8 & $0,00^{* *}$ \\
\hline $\begin{array}{l}\text { Hüftfraktur eines } \\
\text { Elternteils }\end{array}$ & 4,69 & 0,64 & $0,00^{* *}$ & 8,55 & 2,67 & $0,00^{* *}$ \\
\hline $\begin{array}{l}\text { Gegenwärtiges } \\
\text { Rauchen }\end{array}$ & 0,41 & 0,46 & 0,36 & 0,25 & 1,11 & 0,82 \\
\hline $\begin{array}{l}\text { Glukokortikoste- } \\
\text { roide }\end{array}$ & 3,37 & 0,61 & $0,00^{* *}$ & 7,53 & 1,62 & $0,00^{* *}$ \\
\hline $\begin{array}{l}\text { Rheumatoide } \\
\text { Arthritis }\end{array}$ & 0,92 & 0,94 & 0,33 & 5,82 & 2,66 & $0,03^{*}$ \\
\hline $\begin{array}{l}\text { Sekundäre Oste- } \\
\text { oporose }\end{array}$ & 2,1 & 0,38 & $0,00^{* *}$ & 0,66 & 1,05 & 0,53 \\
\hline $\begin{array}{l}\text { Alkohol 3 und } \\
\text { mehr }\end{array}$ & 2,61 & 0,67 & $0,00^{* *}$ & 6,64 & 2,1 & $0,00^{* *}$ \\
\hline $\begin{array}{l}\text { T-Score } \\
\text { Einhei- }\end{array}$ & - & - & - & $-6,44$ & 0,91 & $0,00^{* *}$ \\
\hline \begin{tabular}{l} 
Konstante \\
\hline
\end{tabular} & $-4,60$ & $0,01^{* *}$ & $-2,12$ & 0,61 \\
\hline
\end{tabular}

\subsubsection{Lineares Regressionsmodell für das „FRAX+“-10-Jahresrisiko einer HF ohne und mit BMD}

Im linearen Regressionsmodell für den „FRAX+“-Score für das 10-Jahresrisiko einer HF ohne BMD berechnete sich ein $r^{2}$ von 0,72. Es zeigte sich keine Multikollinearität in diesem Modell. Für insgesamt sechs der neuen Faktoren bestand in diesem Rechenmodell ein hoch signifikanter Einfluss ( $p=0,01)$. Dazu zählten: „Alter“, „BMl“, „vorausgehende Frakturen“, „Glukokortikosteroide“, „sekundäre Osteoporose“, sowie „Alkohol 3 und mehr Einheiten/Tag“. Keinen hoch signifikanten Einfluss hatten die Variablen „Hüftfraktur eines Elternteils“, „gegenwärtiges Rauchen" und „Rheumatoide Arthritis“. Für die Variable "Glukokortikosteroide" errechnete sich der höchste Regressionskoeffizient, Lag dieser Risikofaktor vor, so ergab sich ein Anstieg des Frakturrisikos um 2,06 Prozentpunkt. 
Unter Einbeziehung der Knochendichte berechnete sich ein $r^{2}$ von 0,61. Auch für dieses Modell zeigte sich keine Multikollinearität. Einen hoch signifikanten $(p=0,01)$ Anteil an der erklärten Varianz des Modells hatten die folgenden Faktoren: „Vorausgehende Frakturen“, „Glukokortikosteroide“, „Alkohol 3 und mehr Einheiten/Tag“, „T-Score“. Der höchste Regressionskoeffizient berechnete sich für den Faktor „Alkohol 3 und mehr Einheiten/Tag“.

Tabelle 24: Regressionsmodell für den „FRAX+“-Score einer HF ohne $\left(r^{2}=0,72\right)$ bzw. mit BMD $\left.r^{2}=0,61\right)\left({ }^{* *}\right.$ signifikant auf einem Signifikanzniveau von 1\%; * signifikant auf einem Signifikanzniveau von 5\%)

\begin{tabular}{|c|c|c|c|c|c|c|}
\hline \multirow[t]{2}{*}{ Variablen } & \multicolumn{3}{|c|}{ „FRAX+“ Hüftfraktur ohne BMD } & \multicolumn{3}{|c|}{ „,FRAX+“ Hüftfraktur mit BMD } \\
\hline & $\begin{array}{l}\text { Koeffi- } \\
\text { zient }\end{array}$ & $\begin{array}{l}\text { Robuster Stan- } \\
\text { dardfehler }\end{array}$ & p-Wert & Koeffizient & $\begin{array}{l}\text { Robuster Stan- } \\
\text { dardfehler }\end{array}$ & p-Wert \\
\hline Alter & 0,175 & 0,02 & $0,00^{* *}$ & $-0,49$ & 0,57 & 0,39 \\
\hline BMI & -0.12 & 0,23 & $0,00^{* *}$ & $-0,16$ & 0,12 & 0,17 \\
\hline $\begin{array}{l}\text { Vorausgehende Frak- } \\
\text { turen }\end{array}$ & 1,27 & 0,32 & $0,00^{\star *}$ & 2,72 & 0,92 & $0,00^{\star *}$ \\
\hline $\begin{array}{l}\text { Hüftfraktur eines EI- } \\
\text { ternteils }\end{array}$ & 0,76 & 0,72 & 0,29 & 4,98 & 3,24 & 0,13 \\
\hline $\begin{array}{l}\text { Gegenwärtiges Rau- } \\
\text { chen }\end{array}$ & 0,45 & 0,47 & 0,34 & 0,66 & 1,30 & 0,61 \\
\hline Glukokortikosteroide & 2,06 & 0,65 & $0,00^{\star \star}$ & 6,40 & 1,81 & $0,00^{* *}$ \\
\hline Rheumatoide Arthritis & 0,17 & 0,82 & 0,83 & 4,97 & 2,81 & 0,08 \\
\hline $\begin{array}{l}\text { Sekundäre Osteopo- } \\
\text { rose }\end{array}$ & 1,06 & 0,37 & $0,01^{* *}$ & 0,61 & 1,20 & 0,61 \\
\hline $\begin{array}{l}\text { Alkohol } 3 \text { und mehr } \\
\text { Einheiten/Tag }\end{array}$ & 2,01 & 0,69 & $0,00^{* *}$ & 6,74 & 2,48 & $0,01^{* \star}$ \\
\hline T-Score & - & - & - & $-6,09$ & 1,04 & $0,00^{\star *}$ \\
\hline Konstante & $-6,83$ & 1,43 & $0,00^{\star \star}$ & $-3,1$ & 4,79 & 0,52 \\
\hline
\end{tabular}




\section{Diskussion}

In der vorliegenden Arbeit wurden verschiedene (DVO-, FRAX-, „FRAX+“-Score, Score nach C. Glüer) Frakturrisiko-Evaluationsscores bezüglich ihrer 10-Jahres-Frakturwahrscheinlichkeit, sowie ihrer Therapieempfehlungen für männliche Patienten verglichen. Als Kriterium zur Definition einer Osteoporose, und somit zur Initiierung einer osteoporosespezifischen Therapie wurde 1994 durch die WHO ein T-Score $\leq-2,5$, gemessen an der Lendenwirbelsäule, dem proximalen Femurhals oder Gesamtfemur, postuliert (Kanis et al. 1994; WHO 1991). Die zusätzliche Einbeziehung eines ausgewählten Sets an Risikofaktoren, wie im FRAX- und DVOScore, kann die Genauigkeit der Frakturprognose verbessern (Kanis et al. 2017). Der FRAXScore wurde in mehreren prospektiven Studien validiert und steht in seinen länderspezifischen Formen $80 \%$ der Weltbevölkerung zur Verfügung. Seine einfache Anwendung, ohne oder mit Eingabe der Knochendichtemessung, macht ihn für eine breite Masse zugänglich. Er gleicht jedoch einer "black-box", da nicht ersichtlich ist, welche Gewichtung den eingegebenen Risikofaktoren für die Frakturevaluation beigemessen wird. Zudem gibt es keine offiziellen Therapieschwellen, sondern lediglich länderspezifische Empfehlungen. Bei dem DVO-Score handelt es sich um eine transparente, leicht nachvollziehbare 10-Jahres-Frakturevaluation mit einem breiten Set an Risikofaktoren und einheitlichen Therapieschwellen. Er ist anhand der „FREEDOM-Studie“ validiert (Hadji et al. 2013a).

Zusätzlich wurden in dieser Arbeit zwei nicht publizierte Scores zur Risikoevaluation verwendet. Dabei handelte es sich um eine Erweiterung des FRAX-Scores („FRAX+“), sowie um eine Modifizierung des DVO-Scores nach C. Glüer. Die wichtigsten Unterschiede zwischen den verwendeten Scores fasst Tabelle 25 zusammen.

Tabelle 25: Gegenüberstellung der wichtigsten Gemeinsamkeiten und Unterschiede der verwendeten Scores

\begin{tabular}{|l|l|l|l|}
\hline DVO-Score & Score nach C. Glüer & $\begin{array}{l}\text { FRAX- } \\
\text { Score }\end{array}$ & „FRAX+“-Score \\
\hline Zahlreiche Risikofaktoren entsprechend den DVO-Leitlinien & $\begin{array}{l}\text { 12 Risiko- } \\
\text { faktoren }\end{array}$ & $\begin{array}{l}\text { 12 Risikofaktoren, } \\
\text { erweitertes Set an sekun- } \\
\text { dären Osteoporosen }\end{array}$ \\
\hline Differenzierung der Frakturtypen & „Vorausgehende Fraktur“ \\
\hline $\begin{array}{l}\text { Minimale Knochendichte an LWS oder Femur gesamt O- } \\
\text { DER Femurhals }\end{array}$ & $\begin{array}{l}\text { Minimale Knochendichte Femurhals (fa- } \\
\text { kultativ) }\end{array}$ \\
\hline Mehrstufiges Vorgehen & Einstufiges Vorgehen \\
\hline $\begin{array}{l}\text { Therapieschwelle einheitlich } \\
\text { (>30\%) }\end{array}$ & $\begin{array}{l}\text { Therapieschwelle von >6\% } \\
\text { bzw. >3\% bei einem T- } \\
\text { Score von <-2 }\end{array}$ & Keine einheitliche Therapieschwelle \\
\hline $\begin{array}{l}\text { Zielgröße: 10-Jahres Frak- } \\
\text { turrisiko osteoporseassozi- } \\
\text { ierte Frakturen }\end{array}$ & $\begin{array}{l}\text { Zielgröße: 1-Jahres Fraktur- } \\
\text { risiko osteoporseassoziierte } \\
\text { Frakturen }\end{array}$ & $\begin{array}{l}\text { Zielgröße: 10-Jahres-Frakturrisiko „major } \\
\text { osteoporotic“ (MOF) oder Hüftfraktur (HF) }\end{array}$ \\
\hline
\end{tabular}


Zur weiteren Analyse der FRAX-Scores wurde in der vorliegenden Arbeit ein lineares Regressionsmodell erstellt. Anhand dieses Modells war es möglich die Gewichtung der einzelnen in den FRAX-Score einfließenden Risikofaktoren in dem untersuchten Patientenkollektiv zu ermitteln.

\subsection{Studienkollektiv und Risikofaktorenanalyse}

Für die in der Promotionsarbeit ausgewerteten Daten wurden Patientenakten aus dem Drittzuweiser-Zentrum MVZ endokrinlogikum Göttingen verwendet. Es handelte sich um ein bereits im Vorfeld selektioniertes, überregionales Patientenklientel, das dem MVZ endokrinologikum Göttingen zur osteoporosespezifischen Abklärung zugewiesen wurde. Das Durchschnittsalter der Patienten betrug 58,9 Jahre.

Für männliche Patienten lagen bezüglich der osteoporosespezifischen Auswertung wenige vergleichbare Studien vor. Eine davon ist die „MrOS“-Studie. Bei der „MrOS“-Studie handelt es sich um eine prospektive Studie. Beim Vergleich der Daten der „MrOS“-Studie mit den hier vorliegenden Daten zeigten sich Unterschiede in der Patientenauswahl. In der „MrOS“-Studie wurden patientenbezogene Daten männlicher Patienten über 65 Jahren einbezogen, die sich noch selbstständig versorgen (als "community dwelling“ bezeichnet), keine antiosteoporotische Therapie zum Zeitpunkt des Studienbeginns erhielten und keinen T-Score $\leq-2,5$ (gemessen am Femurhals) aufwiesen. Die „MrOS“-Studie umfasste männliche Patienten aus Hong Kong (1669 Patienten), Schweden (1823 Patienten) und den USA (4365 Patienten). Das Durchschnittsalter variierte in den einzelnen Länderkohorten zwischen 72,4 (Hong Kong) und 75,4 (Schweden) Jahren. Zudem wurden weitere osteoporosespezifische Basisdaten erhoben (Gourlay et al. 2017; Harvey et al. 2017; Orwoll et al. 2005). Ein weitere Analyse osteoporosespezifischer Daten männlicher Patienten findet sich in der Arbeit von Marques et al.. Er untersuchte ein Kollektiv portugiesischer Männer und Frauen. Im Vergleich zur „MrOS“-Studie wurden hierbei männliche Patienten bereits ab einem Alter von 40 Jahren, unabhängig von der gemessenen Knochendichte eingeschlossen (Marques et al. 2017).

Basierend auf den unterschiedlichen Patientenauswahlkriterien zeigte sich in den Daten aus dem MVZ endokrinologikum Göttingen mit 58,9 Jahren ein niedrigeres Durchschnittsalter als in den Vergleichskohorten. In Bezug auf die erhobenen osteoporosespezifischen Risikofaktoren zeigte sich ein prozentual höheres Aufkommen in den Daten aus dem MVZ endokrinologikum Göttingen im Vergleich zur „MrOS“-Studie. So lagen beispielsweise bei $57,8 \%$ der im MVZ endokrinologikum Göttingen untersuchten Patienten „FRAX-relevante“ Frakturen vor. Im „MrOS“-Kollektiv variierte dieser Anteil zwischen 33\% (Schweden) und 13\% (Hong Kong). In dem Patientenkollektiv von Marques et al. lag bei 22,4\% der Patienten eine „FRAX-relevante“Fraktur vor. Auch der durchschnittliche ermittelte T-Score am Femurhals lag im männlichen 
Patientenkollektiv des MVZ endokrinologikum Göttingen mit $-2,1 \pm 1,03$ deutlich niedriger als in den Vergleichsgruppen. Hier variierte er zwischen -1,5 \pm 0,9 (Hong Kong), $-0,6 \pm 1,1$ (USA) und -1,35 \pm 1,4 (Marques et al. 2017). Eine „sekundäre Osteoporose“ lag bei 46,26\% der Patienten des MVZ endokrinologikum Göttingen vor. Aus der „MrOS“-Studie gingen zu diesem Risikofaktor keine Angaben hervor, für die Daten aus Marques et al. lag dieser Risikofaktor bei $6,7 \%$ der Patienten vor. Für die Daten von Marques et al. zeigte sich ein höherer prozentualer Anteil für die Risikofaktoren „gegenwärtiges Rauchen“ (50,4\%), sowie „regelmäßiger Alkoholkonsum“ (42\%) im Vergleich zu den anderen Studienkollektiven. Im Patientenkollektiv des MVZ endokrinologikum Göttingen zeigte sich somit ein höherer prozentualer Anteil sekundärer Osteoporosen als in Vergleichsstudien (Khosla et al. 2008; Marques et al. 2017; NIH 2015). Dies zeigte sich sowohl für die Definition basierend auf dem FRAX-Score, als auch auf den DVO Leitlinien. Für diese Beobachtung lassen sich mehrere mutmaßliche Ursachen finden: Zum einen handelt es sich im Vergleich zu den Kollektiven von Harvey et al. und Marques et al. um stark vorselektionierte Patienten. Das im Durchschnitt niedrigere Patientenalter des Studienkollektives aus dem MVZ endokrinologikum Göttingen mit gleichzeitig vorliegenden niedrigeren T-Scores lässt auf ein höheres Risikoprofil der Patienten schließen. Zudem zeigte sich auch in Studien ein vergleichsweise höherer prozentualer Anteil sekundärer Osteoporosen in männlichen, als in weiblichen Kollektiven (Khosla et al. 2008; NIH 2015). Eine Zusammenfassung der Daten im Vergleich zeigt die Tabelle 26.

Tabelle 26: Vergleich der Basisdaten aus dem MVZ endokrinologikum Göttingen, der „MrOS“-Studie und den Daten von Marques et al.

\begin{tabular}{|c|c|c|c|c|c|}
\hline Basisdaten & $\begin{array}{l}\text { MVZ endokrinologi- } \\
\text { kum Göttingen }\end{array}$ & "MrOS“ & $\begin{array}{l}\text { "MrOS“ } \\
\text { USA }\end{array}$ & $\begin{array}{l}\text { „MrOS“ } \\
\text { Hong } \\
\text { Kong }\end{array}$ & $\begin{array}{l}\text { (Marques et al. } \\
\text { 2017)Marques } \\
\text { et al. }\end{array}$ \\
\hline $\mathbf{N}$ & 147 & 1823 & 4365 & 1669 & 683 \\
\hline Altersdurchschnitt & 58,9 Jahre & 75,4 Jahre & 73,5 Jahre & 72,4 Jahre & 60,3 \\
\hline BMI & $26,67 \mathrm{~kg} / \mathrm{m}^{2}$ & $26,3 \mathrm{~kg} / \mathrm{m}^{2}$ & $\begin{array}{l}27,42 \\
\mathrm{~kg} / \mathrm{m}^{2}\end{array}$ & $23,5 \mathrm{~kg} / \mathrm{m}^{2}$ & \\
\hline $\begin{array}{l}\text { „FRAX-relevante“ Frak- } \\
\text { turen }\end{array}$ & $57,8 \%$ & $33 \%$ & $22 \%$ & $13 \%$ & $22,4 \%$ \\
\hline $\begin{array}{l}\text { Femurfrakturen in der } \\
\text { Familie }\end{array}$ & $9,5 \%$ & $13 \%$ & $17 \%$ & $5 \%$ & $7,3 \%$ \\
\hline $\begin{array}{l}\text { Gegenwärtiges Rau- } \\
\text { chen }\end{array}$ & $25,2 \%$ & $8 \%$ & $3 \%$ & $12 \%$ & $50,4 \%$ \\
\hline $\begin{array}{l}\text { Orale Glukokortikoste- } \\
\text { roide }\end{array}$ & $19,4 \%$ & $2 \%$ & $2 \%$ & $1 \%$ & $5,6 \%$ \\
\hline Rheumatoide Arthritis & $3,4 \%$ & $1 \%$ & $5 \%$ & $1 \%$ & $2,6 \%$ \\
\hline $\begin{array}{l}\text { Regelmäßiger Alkohol- } \\
\text { konsum }\end{array}$ & $14,29 \%$ & $3 \%$ & $4 \%$ & $1 \%$ & $42 \%$ \\
\hline T-Score Femurhals & $-2,1 \pm 1,03$ & $-0,9 \pm 1,0$ & $-0,6 \pm 1,1$ & $-1,5 \pm 0,9$ & $-1,35 \pm 1,4$ \\
\hline $\begin{array}{lr}\begin{array}{l}\text { Sekundäre } \\
\text { seursachen }\end{array} & \begin{array}{r}\text { Osteoporo- } \\
\text { (nach }\end{array} \\
\text { FRAX Kriterien) } & \\
\end{array}$ & $15,65 \%$ & \multicolumn{3}{|c|}{ Keine Angaben } & $6,7 \%$ \\
\hline $\begin{array}{l}\text { Sekundäre } \begin{array}{l}\text { Osteoporo- } \\
\text { seformen (nach DVO } \\
\text { Kriterien) }\end{array} \\
\text { Krien }\end{array}$ & $46,26 \%$ & \multicolumn{3}{|c|}{ Keine Angaben } & Keine Angaben \\
\hline
\end{tabular}


In dem anhand der Patientendaten des MVZ endokrinologikum Göttingen erstellen linearen Regressionsmodells zeigte sich, dass eine Vielzahl von Risikofaktoren einen hoch signifikanten Einfluss auf das 10-Jahresfrakturrisko der FRAX-Scores in dem untersuchten Kollektiv hatten: Für das FRAX-Modell ohne Einbeziehung der Knochendichtemessung für eine „major osteoporotic“ Fraktur hatten alle berücksichtigen Faktoren außer "gegenwärtiges Rauchen“ und „Rheumatoide Arthritis“ auf einem Signifikanzniveau von einem Prozent einen hochsignifikanten Einfluss. Die identischen Risikofaktoren hatten einen hoch signifikanten Einfluss auf das „FRAX+“-Modell für eine "major osteoporotic“ Fraktur. Im Vergleich zeigte sich eine stärkere Gewichtung des Faktors „sekundäre Osteoporosen” im „FRAX+“-Modell (Regressionskoeffizienten: FRAX: 1,07; „FRAX+“: 2,1). Lag ein sekundäre Osteoporose vor, so stieg das 10Jahres-Frakturrisiko um 1,07\% (FRAX) bzw. 2,1\% („FRAX+“) an. Die übrigen Risikofaktoren zeigten geringfügigere Differenz der Regressionskoeffizienten im FRAX- und „FRAX+“-Modell.

Einen hoch signifikanten Einfluss auf alle FRAX-Scores, sowohl mit, als auch ohne Knochendichtemessung, hatten die Faktoren „vorausgehende Frakturen“, „orale Glukokortikosteroide“ und „Alkohol 3 und mehr Einheiten/Tag“. In Studien zeigte sich, dass eine vorliegende Fraktur das Risiko für weitere Frakturen erhöht. Im Falle einer stattgehabten Wirbelkörperfraktur erhöht sich das Risiko für weitere Wirbelkörperfrakturen um das bis zu vierfache. Das Risiko einer Hüftfraktur erhöht sich um das bis zu zweifache bei stattgehabter Wirbelkörperfraktur. Nur ein geringer, nicht signifikanter Anteil des erhöhten Risikos ist dabei durch die Varianz der Knochendichte zu erklären. Der Faktor ist somit weitgehend unabhängig von der Knochendichtemessung (Kanis et al. 2004a; Lems 2007). In der linearen Regressionsanalyse zeigte sich analog zu diesen Ergebnissen ein von der Knochendichtemessung unabhängig Einfluss des Faktors „Vorausgehende Frakturen“. Unter Einbeziehung der Knochendichte errechneten sich jedoch höhere Regressionskoeffizienten als unter Auslassung der Knochendichte. Somit wurde das 10-Jahres-Frakturrisiko unabhängig von der Knochendichtemessung durch den Parameter „vorausgehende Frakturen“ beeinflusst. Bezog man die Knochendichte jedoch mit ein, so zeigte sich bei stattgehabter Fraktur ein stärker Anstieg des 10-Jahres-Frakturrisikos als unter Auslassung der Knochendichtemessung.

Für die Risikofaktoren „Alkohol 3 und mehr Einheiten/Tag“, sowie „orale Glukokortikosteroide“ zeigten sich im Vergleich zum untersuchten Patientenkollektiv konforme Beobachtungen in verschiedenen Studien (Kanis et al. 2005; Kanis et al. 2004c; Kanis et al. 2007; Pluijm et al. 2009; Zhang et al. 2015). Auch hier wird ein von der Knochendichtemessung unabhängiger Einfluss dieser Faktoren auf das Frakturrisiko beschrieben.

Der Risikofaktor „Hüftfraktur eines Elternteils“ hatte unabhängig von der gemessenen Knochendichte einen hoch signifikanten Einfluss auf das FRAX- bzw. „FRAX+“-Modell für eine „major osteoporotic“ Fraktur. Der hohe Einfluss dieses Risikofaktors war möglicherweise in 
dem niedrigen Durchschnittsalter des Patientenkollektivs (58,9 Jahre) begründet. Studien belegen einen mit zunehmendem Alter schwindende Einfluss auf die 10-Jahres-Frakturwahrscheinlichkeit. Für eine Hüftfraktur zeigte sich ein signifikanter Einfluss auf das Frakturrisiko bis zu einem Alter von 80 Jahren, für alle anderen Frakturen bis zu einem Alter von 75 Jahren (Kanis et al. 2004b). Widersprüchlich dazu war, dass sich trotz des niedrigen Durchschnittsalters im untersuchten Patientenkollektiv kein signifikanter Einfluss dieses Risikofaktors auf das 10-Jahres-Frakturrisiko für eine Hüftfraktur zeigte. Folglich ist davon auszugehen, dass andere Faktoren einen stärkeren Einfluss auf das hier vorliegende Modell hatten und dieser Faktor somit nur einen nachrangigen Einfluss hatte. In der Literatur ließen sich keine vergleichbaren Aussagen finden.

Bemerkenswert war, dass die Faktoren „Alter“, „BMl“, sowie „sekundäre Osteoporose“ keinen signifikanten Einfluss mehr auf die FRAX- und „FRAX+“-Modelle hatten, sofern die Knochendichtemessung miteinbezogen wurde. Dies war überraschend, da das Alter in verschiedenen Studien sowohl in einfachen, als auch komplexen Scores als einer der stärksten Risikofaktoren beschrieben wurde. Das Alter allein betrachtet hatte in diesen Studien eine Sensitivität von $90 \%$ für eine Hüftfraktur und "major osteoporotic“ Fraktur bei Patienten $>70$ Jahren (Rubin et al. 2013a). Auch für diese zu Studien widersprüchliche Beobachtung könnte das geringe Durchschnittsalter der Patienten mitbegründend sein. Bei jüngeren Patienten zeigte sich, dass eine bessere Frakturprognose unter Verwendung zusätzlicher Risikofaktoren zum Alter erzielt wird (Kanis et al. 2007). Demnach gilt es, bei Verwendung des FRAX-Scores die Altersgrenzen kritisch zu überdenken. Es zeigte sich im untersuchten Kollektiv männlicher Patienten, dass der FRAX-Score das Alter in Kombination mit dem hohen patientenbezogenem Risikoprofil nicht adäquat dargestellt hat.

Für den Risikofaktor „sekundäre Osteoporose“ zeigte sich unter Einbeziehung zusätzlicher Risikofaktoren im „FRAX+“-Modell kein signifikanter Einfluss unabhängig von der Knochendichtemessung. Diese Beobachtung war zu erwarten, da der FRAX-Algorithmus sich nicht veränderte und somit die Wichtung dieses Faktors im FRAX-Algorithmus unverändert blieb. Im Vergleich zum FRAX-Modell wurden die folgenden sekundären Osteoporseursachen in das „FRAX+“-Modell eingeschlossen: gastrointestinale Erkrankungen, Morbus Crohn, Endokrinopathien, Hyperkalzurie, Antiepileptika, Thyroxin-Einnahme, Hypokalzurie, primärer Hyperparathyreodismus, Genetik, Zustand nach Transplantation, Hyperthyreose, Metastasen, VitaminD-Mangel, lokalisierte Osteoporose, Heparin/Marcumar- Einnahme und Mastozytose. Zu erwarten wäre durch die zusätzliche Berücksichtigung von Risikofaktoren, wie sie sich auch im DVO-Score und Score nach C. Glüer, wiederfinden, eine Annäherung des „FRAX+“-Scores an diese beiden Scores bezüglich der 10-Jahres-Frakturrisiken. Dies zeigte sich jedoch im untersuchten Patientenkollektiv nicht. Die Einbeziehung zusätzlicher Risikofaktoren in das 
„FRAX+“-Modell führte jedoch im Vergleich zum FRAX-Modell zu keiner signifikant höheren Korrelation mit dem DVO- Score bzw. Score nach C. Glüer. Es zeigte sich weiterhin ein hochsignifikanter Unterschied der zentralen Aussagetendenzen zwischen dem „FRAX+“- Score und dem Score nach C. Glüer bzw. DVO-Score. Demnach scheinen andere Faktoren in den FRAX-Modellen stärker gewichtet zu sein. Die zusätzliche Eingabe von sekundären Osteoporoseursachen führte somit zu keiner weiteren Annährung der Scores des „FRAX+“-Scores an den DVO-Score bzw. Score nach C. Glüer. Unter Verwendung der Knochendichtemessung war der Einfluss anderer Risikofaktoren so dominant, dass der Risikofaktor „sekundäre Osteoporose" gar keinen signifikanten Einfluss mehr auf die FRAX-Modelle hatte.

\subsection{0-Jahres-Frakturrisiken im Vergleich}

Vergleicht man die durchschnittlichen FRAX-10-Jahres-Risiken mit den Frakturrisiken aus der „MrOS“-Studie, so zeigten sich in dieser Arbeit jeweils höhere 10-Jahres-Frakturrisiken ohne Einbeziehung der Knochendichtemessung als mit Einbeziehung der Knochendichtemessung. Die Ergebnisse aus dem MVZ endokrinologikum Göttingen zeigten ein gegensätzliches Verhalten: Unter Einbeziehung der Knochendichte berechneten sich höhere durchschnittliche Frakturrisiken (Tabelle 27). Diese Beobachtung unterschied sich von anderen Studien, in denen sich unter Einbeziehung der Knochendichtemessung ein geringerer FRAX-Score berechnete. Dies zeigte sich sowohl in männlichen als auch weiblichen Patientenkollektiven (Ettinger et al. 2013; Harvey et al. 2017; Leslie et al. 2010; Tamaki et al. 2011). Auch in dem männlichen Studienkollektiv von Marques et al. zeigten sich unter Einbeziehung der Knochendichtemessung höhere Mediane für die 10-Jahres-Frakturrisiken (Tabelle 28). Auch das Patientenkollektiv von Marques et al. wies, wie auch die Patienten aus dem MVZ endokrinologikum Göttingen, ein niedrigeres durchschnittlicheres Alter (60,3 Jahre) und einen niedrigeren durchschnittlichen T-Score $(1,35 \pm 1,4)$ im Vergleich zum „MrOS“ Studienkollektiv auf (Harvey et al. 2017; Marques et al. 2017). Auffällig war jedoch, dass trotz des vergleichsweise höheren Risikoprofils der Patienten des MVZ endokrinologikum Göttingen die durchschnittlichen Frakturrisiken ohne Einbeziehung der Knochendichte geringer waren, als in der „MrOS“-Studie. Eine Begründung könnte das höhere Durchschnittalter in der „MrOS“-Studie sein. Diese Beobachtungen waren unabhängig von der Ethnie. Dabei ist anzumerken, dass der Ethnienunterschied bezüglich des Frakturrisikos bei Männern geringer ausgeprägt ist, als bei Frauen (Cauley 2011; Shin et al. 2014). Trotzdem zeigte sich in Studien eine höhere Frakturprävalenz für hellhäutige Männer, als für Asiaten oder Afroamerikaner. Auch in den vorliegenden Daten zeigt sich diese Beobachtung: Die höchsten Frakturwahrscheinlichkeiten berechneten sich für das schwedische Patientenkollektiv der „MrOS“ Studie bzw. für die Daten aus dem MVZ endokrinologikum, sofern man die Knochendichtemessung miteinbezog. 
Tabelle 27: Vergleich der durchschnittlichen FRAX-10-Jahres-Frakturrisken

\begin{tabular}{|c|c|c|c|c|c|}
\hline \multirow{2}{*}{$\begin{array}{l}\text { Durchschnittliche } \\
\text { FRAX 10-Jahres- } \\
\text { Frakturrisiken }\end{array}$} & \multicolumn{2}{|c|}{$\begin{array}{l}\text { MVZ endokrinologikum } \\
\text { Göttingen }\end{array}$} & \multirow[t]{2}{*}{$\begin{array}{l}\text { „MrOs“" } \\
\text { Schweden }\end{array}$} & \multirow[t]{2}{*}{$\begin{array}{l}\text { „MrOS“" } \\
\text { USA }\end{array}$} & \multirow[t]{2}{*}{$\begin{array}{l}\text { "MrOS“ } \\
\text { Hong Kong }\end{array}$} \\
\hline & FRAX-Score & $\begin{array}{l}\text { „FRAX-+“- } \\
\text { Score }\end{array}$ & & & \\
\hline MOF ohne BMD & $6,2 \pm 3,75$ & $6,62 \pm 4,36$ & $13,5 \pm 6,2$ & $9,2 \pm 5,0$ & $6,9 \pm 2,9$ \\
\hline HF ohne BMD & $1,97 \pm 2,5$ & $2,24 \pm 2,97$ & $7,5 \pm 5,5$ & $3,7 \pm 4,0$ & $3,4 \pm 2,6$ \\
\hline MOF mit BMD & $11,8 \pm 11,03$ & $11,8 \pm 11,03$ & $11,4 \pm 6,8$ & $7,9 \pm 4,8$ & $6,7 \pm 3,3$ \\
\hline HF mit BMD & $6,8 \pm 10,6$ & $6,8 \pm 10,6$ & $5,6 \pm 6,1$ & $2,5 \pm 3,6$ & $3,1 \pm 2,7$ \\
\hline
\end{tabular}

Tabelle 28: Vergleich der Mediane und 25-\% bzw. 75-\% Interquartilenabstände (IQR)

\begin{tabular}{|l|l|l|l|}
\hline \multirow{2}{*}{$\begin{array}{l}\text { Median der FRAX 10-Jahres- } \\
\text { Frakturrisiken (IQR) }\end{array}$} & \multicolumn{2}{|l|}{ MVZ endokrinologikum Göttingen } & \multirow{2}{*}{ Marques et al. } \\
\cline { 2 - 3 } & FRAX-Score & „FRAX-4“-Score & \\
\hline MOF ohne BMD & $5(3,2-7,7)$ & $5,3(3,4-8,7)$ & $1,6(1,6-4,4)$ \\
\hline HF ohne BMD & $1,1(0,4-2,75)$ & $1,1(0,4-3,05)$ & $0,5(1,7-6)$ \\
\hline MOF mit BMD & $8,5(5,9-14,75)$ & $8,5(5,9-14,75)$ & $3,1(0,2-1,6)$ \\
\hline HF mit BMD & $3,35(1,73-8,5)$ & $3,35(1,73-8,5)$ & $0,95(0,28-2,93)$ \\
\hline
\end{tabular}

Im untersuchten Kollektiv männlicher Patienten zeigte sich für alle verwendeten FRAX-Scores jeweils ein signifikant höheres durchschnittliches Frakturrisiko bzw. höhere Median für eine „major osteoporotic“ Fraktur, als für eine Hüftfraktur im Vergleich zu den anderen männlichen Studienkollektiven. Unter Einbeziehung der Knochendichtemessung errechneten sich nochmals signifikant höhere durchschnittliche 10-Jahres-Frakturrisiken. Ein möglicher Erklärungsansatz dafür ist der im Durchschnitt sehr geringe T-Score im untersuchten Kollektiv in Kombination mit dem aus der linearen Regressionsanalyse hervorgehenden hoch signifikanten Einfluss der Knochendichtemessung. Für den FRAX- und „FRAX+“-Score waren die 10-JahresFrakturrisiken unter Einbeziehung der Knochendichtemessung identisch (jeweils $11,8 \%$ für eine MOF und 6,8\% für eine HF). Diese Beobachtung lässt sich anhand der Regressionsanalyse nachvollziehbar. Hier zeigte sich, dass der Faktor "sekundäre Osteoporose“ unter Einbeziehung der Knochendichtemessung keinen signifikanten Einfluss mehr auf die „FRAX“-Modelle hatte. Dementsprechend zeigte sich auch kein Unterschied in den ermittelten Frakturrisiken. Die zusätzliche Verwendung der Knochendichtemessung machte im untersuchten Patientenkollektiv demnach die Eingabe des Faktors „sekundäre Osteoporseursachen“ überflüssig. Der Faktor hatte demnach einen von der Knochendichtemessung abhängigen Einfluss auf das FRAX-Modell.

Auch in Studien und Metaanalyen zeigte sich, dass die meisten im FRAX-Algorithmus verwendeten sekundären Osteoporoseursachen einen von der Knochendichte abhängigen Einfluss auf das Frakturrisiko haben. Dazu zählen die Faktoren: Mangelernährung und Malabsorption 
mit daraus resultierendem Untergewicht (De Laet et al. 2005), Diabetes Mellitus Typ I (Vestergaard 2007) subklinische und manifeste Hyperthyreose (Yan et al. 2016) und Osteogenesis imperfecta (Espallargues et al. 2001). Eine nach Adjustierung von der Knochendichte jedoch unabhängiger Anstieg des Frakturrisikos um das 1,2- bis 1,3-fache zeigte sich in Studien für den Faktor Hypogonadismus (Leitlinie Osteoporose 2017; Espallargues et al. 2001). Dieser von der Knochendichte unabhängiger Einfluss auf das Frakturrisiko konnte in dem vorliegenden Patientenkollektiv nicht bestätigt werden. Für die sekundäre Osteoporoseursachen Leberzirrhose fand sich unabhängig der Ätiologie keine ausreichende Datenlage. Für eine alkoholische Leberzirrhose zeigte sich in Studien keine Veränderung der Knochendichte gegenüber Kontrollgruppen, jedoch ein erhöhtes Frakturrisiko (Zhang et al. 2015).

Da für das in dieser Arbeit untersuchte männlichen Patientenkollektiv aus dem MVZ endokrinologikum Göttingen keine Follow-up Daten vorlagen, können im Folgenden nur die Frakturhäufigkeiten aus der Literatur diskutiert werden. Bei der „MrOS“-Studie und der Studie von Marques et al. handelt es sich um epidemiologische, prospektive Studien. Patienten wurden im Schnitt über einen Zeitraum von 10-Jahren verfolgt. Dabei zeigte sich, dass der FRAXScore für eine Hüftfraktur ohne Einbeziehung der Knochendichte für das untersuchte männliche Kollektiv am genausten das tatsächliche Frakturrisiko prognostizierte. Das lässt vermuten, dass der FRAX-Score ohne Einbeziehung der Knochendichte allein durch die Kombination der eingeschlossenen Risikofaktoren das tatsächliche Frakturrisiko am besten vorhersagte. Basierend auf der Beobachtung dieser Studie würde im von uns untersuchten Kollektiv männlicher Patienten das 10-Jahres-Frakturrisiko anhand des DVO-Scores und FRAX-Scores für eine HF unter Einbeziehung der Knochendichtemessung überschätzt werden. Dabei gilt es jedoch zu beachten, dass es sich bei dem von uns untersuchten Kollektiv um kein epidemiologisches Patientengut handelte. Das von uns untersuchte Kollektiv war jünger, wies einen deutlich niedrigeren T-Score auf und hatte zudem ein höheres Risikoprofil. Insgesamt zeigte sich in der Studie nach Marques et al. der FRAX-Score für Männer besser als für Frauen kalibriert (Marques et al. 2017). Im weiblichen Kollektiv zeigte sich eine Überschätzung des Risikos für eine Hüftfraktur, sowie eine Unterschätzung des Risikos für eine „major osteoporotic“ Fraktur. Bezüglich der Frakturprognose war der FRAX-Score besser kalibriert als die Knochendichtemessung alleinig betrachtet (Marques et al. 2017).

Unter Einbeziehung der Knochendichte zeigte sich wider Erwarten keine Verbesserung der Genauigkeit des FRAX-Modells in der „MrOS“-Studie. Es ergab sich zwar eine höhere Genauigkeit der Frakturprognose für die Patienten ohne Frakturen, das Risiko der Patienten, die eine Fraktur erlitten, wurde jedoch überschätzt (Ettinger et al. 2013). Diese Beobachtung zeigte sich auch in anderen Studien, die männliche und weibliche Patienten einbezogen (Marques et al. 2017; Tamaki et al. 2011). Dieses Ergebnis war überraschend, da der FRAX-Score 
basierend auf der Knochendichtemessung am Femurhals kalibriert wurde. Knochendichtemessungen am Femurhals zeigten eine gute Vorhersagekraft für Schenkelhalsfrakturen (Leslie et al. 2011). Demnach wäre eine genauere Frakturprognose für das Ereignis einer Hüftfraktur unter Einbeziehung der Knochendichte zu erwarten gewesen. Dies zeigte sich nicht in der „MrOS“-Studie. Eine Begründung dafür könnten die niedrigen durchschnittlichen TScores (zwischen-0,6 und-1,5) sein. Der FRAX-Sore für eine „major osteoporotic“ Fraktur zeigte ohne Einbeziehung der Knochendichtemessung in der „MrOS“-Studie eine schlechtere Vorhersage der tatsächlich aufgetretenen Frakturen als der FRAX-Score für eine Hüftfraktur. Im Vergleich überschätzte er die tatsächliche Frakturhäufigkeit im Kollektiv. Auch unter Einbeziehung der Knochendichtemessung zeigte sich für das Ereignis einer "major osteoporotic“ Fraktur keine Verbesserung der Genauigkeit der Frakturprognose (Ettinger et al. 2013). Für das Ereignis einer Wirbelkörperfraktur wäre eine bessere Aussagekraft des FRAX-Scores denkbar, sofern man den gemessene T-Score an der Lendenwirbelsäule miteinbezieht. Dies konnte jedoch in großen epidemiologischen Studien nicht belegt werden (Kanis et al. 2006; Leslie et al. 2007). Um für das Patientenkollektiv des MVZ endokrinologikum Göttingen eine Aussage treffen zu können, welcher der Scores die tatsächlich aufgetretenen Frakturen besser abbildet, müssten Follow-up Daten zur Frakturhäufigkeit erhoben werden. Dabei ist bei einem Vergleich mit anderen Studienkollektiven zu berücksichtigen, dass es sich bei den Daten aus dem MVZ endokrinologikum Göttingen um ein vorselektioniertes Patientenkollektiv handelt. Einer der markantesten Unterschiede der im Rahmen dieser Arbeit analysierten Scores ist das verwendete Set bzw. dessen Umfang an Risikofaktoren. In verschiedenen Publikationen zeigte sich jedoch, dass komplexere Scores im Vergleich zu einfachen Scores, die beispielsweise nur das Alter und Gewicht erfassen, nicht genauer in der Prognose von osteoporoseassoziierten Frakturen sind (Ensrud et al. 2009; Rubin et al. 2013b).

In unserem Patientenkollektiv des MVZ endokrinologikum Göttingen zeigte sich im Vergleich der 10-Jahres-Frakturrisiken der FRAX-Scores und des DVO-Scores nur unter Einbeziehung der Knochendichte eine hochsignifikant, gering positive Korrelation. Zwischen allen FRAXScores ohne Einbeziehung der Knochendichtemessung (sowohl für das Ereignis einer „major osteoporotic" Fraktur, als auch Hüftfraktur) zeigte sich keine signifikante Korrelation mit dem DVO-Score. Dies spiegelte sich in den errechneten 10-Jahres-Frakturrisiken wider. Bei $61,09 \%$ lag ein 10-Jahresfrakturrisiko $>30 \%$ basierend auf dem DVO-Score vor. In den FRAXScores errechnete sich nur unter Einbeziehung der Knochendichtemessung für das Ereignis einer „major osteoporotic“ Fraktur bzw. einer Hüftfraktur ein über 30\%iges Frakturrisiko für je 3,9\% der Patienten. Das durchschnittliche FRAX-10-Jahres-Frakturrisiko für diese Patienten mit einem laut dem DVO-Score über 30\%igem Frakturrisiko lag zwischen 6,92\% (MOF) und $2,47 \%$ (HF). Dabei gilt zu berücksichtigen, dass die Scores sich nicht nur bezüglich der eingeschlossenen Risikofaktoren, sondern auch der gewählten Therapiegrenzen unterscheiden. 
Diese liegen laut Metaanalysen länderspezifischer Osteoporoseleitlinien für den FRAX-Score für eine „major osteoporotic" Fraktur bei einem 10 Jahres-Frakturrisiko $\geq 20 \%$ bzw. $\geq 3 \%$ für eine Hüftfraktur. Basierend auf den DVO-Leitlinien wird für den FRAX-Score eine Therapiegrenze von einem 10-Jahres-Frakturrisiko $\geq 14 \%$ für eine „major osteoporotic“ Fraktur und $\geq 3 \%$ für eine Hüftfraktur empfohlen (Kanis et al. 2016a). Diese Anpassung der Therapiegrenze für eine „major osteoporotic“ Fraktur geht unter anderem auf die vorliegenden Daten des MVZ endokrinologikum Göttingen zurück. Auch andere europäische Länder geben Therapiegrenzen für eine „major osteoporotic“ Fraktur unter 20\% an. Dazu zählen die Schweiz (Therapiegrenze für eine „major osteoporotic“ Fraktur von 15\%), Spanien (Therapiegrenze für eine „major osteoporotic“ Fraktur von 11\%) und Schweden (Therapiegrenze für eine „major osteoporotic" Fraktur 15\% oder 20\%) (Kanis et al. 2016a). Der direkte Vergleich der 10-Jahres-Frakturrisiken erscheint somit unter Einbeziehung der Therapiegrenzen aussagekräftiger. Nähere Ausführungen dazu finden sich unter dem Punkt 5.3.

Der Score nach C. Glüer wurde basierend auf den DVO-Leitlinien 2014 entwickelt. Im Vergleich zum DVO-Score bezieht er sich jedoch nicht auf ein 10- Jahres-, sondern 1-Jahresfrakturrisiko. Durch die Hochrechnung auf ein 10-Jahres-Frakturrisiko ist mit einer Überschätztung durch die fehlende Einkalkulierung der Mortalität zu rechnen. Für den Score nach C. Glüer zeigte sich im untersuchten männlichen Kollektiv des MVZ endokrinologikum Göttingen eine hochsignifikante positive Korrelation sowohl mit dem DVO-Score, als auch mit allen FRAXund „FRAX+“-Scores. Die stärkste Korrelation bestand zwischen dem FRAX-Score für eine „major osteoporotic“ Fraktur ohne BMD. Der Score nach C. Glüer hatte eine höhere Übereinstimmung mit den FRAX-Scores für eine „major osteoporotic“ Fraktur als mit dem DVO-Score. Dieses Ergebnis ist überraschend in Anbetracht der Tatsache, dass der DVO-Score und Score nach C. Glüer ein gemeinsames Set an Risikofaktoren basierend auf den DVO-Leitlinien von 2014 aufweisen. Demnach muss die Ursachen für die Unterschiede in den Frakturprognosen in der Wichtung der Risikofaktoren begründet sein. Zudem ist die Hochrechnung des Scores nach C. Glüer auf ein 10-Jahres-Frakturrisiko nicht zu vernachlässigen.

\subsection{Therapieempfehlungen}

Für den DVO-Score existiert eine feste Therapiegrenze. Sie liegt bei einem 10-Jahres-Frakturrisiko $>30 \%$. Im untersuchten Patientenkollektiv erhielten darauf basierend $61,9 \%$ der Patienten eine positive Therapieempfehlung. Der DVO-Score ist anhand des weiblichen Patientenkollektivs der „FREEDOM“-Studie validiert. Es zeigte sich, dass 80\% (in Abhängigkeit der Subgruppen zwischen $81 \%$ und 92,4\%) der Patientinnen dieser Studie auch anhand des DVOScores als therapiebedürttig eingestuft wurden. Es wurden nicht alle Patienten als therapiebedürftig eingestuft. Basierend auf diesen Daten wäre eine Anpassung des Schwellenwertes der Therapiegrenze der DVO-Leitlinien nach unten zu diskutieren (Hadji et al. 2013a). 
Auch der modifizierte Score nach C. Glüer gibt feste Therapiegrenzen vor. Sie liegen bei einem 1-Jahres-Frakturrisiko $>6 \%$ bzw. $>3 \%$ bei einem T-Score $<-2$ SD. Dieser Score ist noch nicht validiert. Im Vergleich mit dem DVO-Score zeigte sich trotz der Parallelen in dem Set an Risikofaktoren ein hoch signifikanter Unterschied in den Therapieentscheidungen. Eine patientenbezogene Übereinstimmung der Therapieentscheidungen lag bei 53,74\% der Patienten vor. In Bezug auf die Therapieentscheidung „ja“, stimmten die Scores bei 19\% der Patienten überein. Obwohl der prozentuale höhere Anteil an Patienten durch den DVO-Score als therapiebedürftig eingestuft wurde, gab es dennoch 3,4\% Patienten, die durch den Score nach C. Glüer, aber nicht durch den DVO-Score als therapiebedürftig eingestuft wurden. Eine mögliche Erklärung für diese Differenzen könnte in der unterschiedlichen Gewichtung der Risikofaktoren im Score nach C. Glüer liegen. Im Vergleich zum DVO-Score liegt eine Einteilung in moderate, starke und sehr starke Risikofaktoren vor. Das 1-Jahres-Frakturrisiko ergibt sich aus der Kombination von Risikofaktoren. Dabei werden zwischen null und maximal einem starken und einem sehr starken Risikofaktor in Kombination mit der Knochendichte und dem Alter berücksichtigt. Zur Überprüfung, ob die anhand des Scores nach C. Glüer berechneten 1-JahresFrakturrisiken das tatsächliche Frakturkommen widerspiegeln oder gegebenenfalls eine weitere Anpassung notwendig ist, wäre eine Patientenbefragung bezüglich der stattgehabten Frakturen im Jahr nach der Erstvorstellung denkbar.

Für den FRAX-Score liegen keine einheitlichen Therapiegrenzen vor. In Meta-Analysen länderspezifischer Leitlinien waren die am häufigsten verwendeten Grenzen $\geq 3 \%$ für eine Hüftfraktur, sowie $\geq 20 \%$ für eine „major osteoporotic" Fraktur. Für Deutschland wird eine Therapiegrenze von $\geq 14 \%$ für eine „major osteoporotic“ Fraktur und $\geq 3 \%$ für eine Hüftfraktur angegeben (Leitlinie Osteoporose 2017). Unabhängig von der gewählten Therapiegrenze von $\geq 14 \%$ bzw. $\geq 20 \%$ zeigte sich im untersuchten Kollektiv kein Unterschied in der Anzahl der therapierten Patienten, sofern man die FRAX-Scores für eine „major osteoporotic“ Fraktur und eine Hüftfraktur zusammenbetrachtete. Alle Patienten, die durch die FRAX-Scores für eine Hüftfraktur als therapiebedürftig eingestuft wurden, erhielten auch eine Therapieempfehlung basierend auf den Scores für eine MOF (unabhängig von er gewählten Therapiegrenze von $\geq 14 \%$ bzw. $\geq 20 \%$ ).

Im FRAX-Score zeigte sich unter Einbeziehung der Knochendichte zwischen den gewählten Therapiegrenzen von $\geq 14 \%$ bzw. $\geq 20 \%$ für eine "major osteoporotic" Fraktur ein signifikanter Unterschied. Für den „FRAX+“-Score zeigte sich sowohl mit als auch ohne Knochendichte ein hochsignifikanter Unterschied zwischen den gewählten Therapiegrenzen von $\geq 14 \%$ bzw. $\geq 20 \%$ für eine „major osteoporotic“ Fraktur. Demnach gilt es sorgfältig abzuwägen welche Therapiegrenze man für die FRAX-Score wählt. 
In der Analyse des männlichen Studienkollektivs zeigten sich kein signifikanter Unterschied in den Therapieentscheidungen zwischen dem DVO-Score und dem FRAX- bzw. "FRAX+“Score für eine Hüftfraktur unter Einbeziehung der Knochendichte. Für alle anderen untersuchten Scores zeigte sich ein hochsignifikanter Unterschied zwischen den Therapieentscheidungen in Bezug auf den DVO-Score. Für den DVO-Score und den FRAX-Score für eine Hüftfraktur unter Einbeziehung der Knochendichtemessung errechnete sich eine Übereinstimmung von insgesamt 64,6\% aller Therapieentscheidungen (ja und nein), und 40,8\% in Bezug auf die Therapieentscheidung „ja“. Auch hier gab es wiederum Patienten, die zwar durch den DVOScore als therapiebedürftig eingestuft wurden, jedoch nicht durch den FRAX-Score (20\%) und andersherum (15,4\%). Insgesamt erhielten 56,2\% der Patienten eine positive Therapieempfehlung basierend auf dem FRAX- bzw. „FRAX+“-Scores für eine Hüftfraktur unter Einbeziehung der Knochendichtemessung. Ohne Verwendung der Knochendichte erhielten basierend auf dem FRAX-Score für eine Hüftfraktur nur 22,4\% bzw. 26,5\% basierend auf dem „FRAX+“Score eine positive Therapieempfehlung. Die wenigsten positiven Therapieempfehlungen ergaben sich mit 0,7\% basierend auf dem FRAX-Score für eine „major osteoporotic“ Fraktur mit einer Therapiegrenze von $\geq 20 \%$. Dies erschien im Vergleich mit vorliegender Literatur verwunderlich, da Anhand retrospektiver Studien eine gute Frakturprognose für diesen Score in männlichen Kollektiven ermittelt wurde (Marques et al. 2017).

Wie bereits angeführt hat sich der FRAX-Score für eine Hüftfraktur ohne Einbeziehung der Knochendichtemessung in prospektiven Studien als der am besten für die Frakturprognose kalibrierte Score gezeigt (Ettinger et al. 2013). Demnach würden im Vergleich zum DVO-Score im Studienkollektiv des MVZ endokrinologikum Göttingen 39,5\% an Patienten weniger therapiert werden. Aufgrund dieser großen Differenz in den Therapieempfehlungen sollte eine Follow-up Studie der vorliegenden Patientendaten aus dem MVZ endokrinologikum Göttingen durchgeführt werden, um eine Aussage treffen zu können, welcher der Scores eine bessere Frakturprognose in einem vorselektionierten Patientenkollektiv erlaubt.

Im Weiteren stellt sich die Frage, wie die FRAX-Therapiegrenzen der im Rahmen der Arbeit ausgewerteten männlichen Patientendaten aussehen müssten, damit durch die FRAX-Scores die gleichen Patienten therapiert werden, wie durch den DVO-Score mit einem 10-JahresFrakturrisiko $>30 \%$. Die Auswertung hat gezeigt, dass sich die Therapiegrenzen im untersuchten Kollektiv sowohl bezüglich der gewählten Zielgröße „major osteoporotic“ Fraktur bzw. Hüftfraktur, als auch in Abhängigkeit der Knochendichtemessung unterschieden. Demnach errechnete sich ohne Berücksichtigung der Knochendichtemessung für eine "major osteoporotic“ Fraktur eine Therapieschwelle von $\geq 6,92 \%$. Sie liegt damit deutlich niedriger als die in Metaanalysen angegebene Schwelle von $\geq 20 \%$ bzw. $\geq 14 \%$. Für das Ereignis einer Hüftfraktur lag die in der Arbeit ermittelte Therapieschwelle ohne Einbeziehung der Knochendichtemessung 
mit $\geq 2,47 \%$ nah an der in Metaanalysen angegeben Grenze von $\geq 3 \%$. Ohne Einbeziehung der Knochendichte stimmten die errechneten Therapiegrenzen basierend auf den Daten des MVZ endokrinologikum Göttingen besser mit Therapiegrenzen aus der Literatur für eine Hüftfraktur als für eine „major osteoporotic“ Fraktur überein. Unter Einbeziehung der Knochendichtemessung verschob sich die anhand der Daten des MVZ endokrinologikum Göttingen berechneten Therapiegrenzen nach oben. Für eine „major osteoporotic“ Fraktur errechnete sich eine Therapiegrenze von $\geq 14,43 \%$. Sie lag somit nahe der von der DVO angegebenen Therapiegrenze von $\geq 14 \%$. Die basierend auf dem männlichen Patientenkollektiv errechnete Therapiegrenze für eine Hüftfraktur unter Einbeziehung der Knochendichtemesung lag mit $\geq 9,18 \%$ weit über der angegebenen Grenze von $\geq 3 \%$. In dem stark vorselektionierten männlichen Patientenkollektiv des MVZ endokrinologikum Göttingen variierten die Therapiegrenzen somit stark in Abhängigkeit der Knochendichtemessung. Dies lässt sich durch die niedrigen durchschnittlichen T-Scores $(-2,1 \pm 1,03)$ erklären.

Basierend auf den ausgewerteten Daten zeigten sich demnach teils starke Abweichungen der ermittelten Therapiegrenzen von den in Metaanalysen bzw. von der DVO empfohlenen Grenzen. Zudem zeigte sich eine Veränderung der Therapiegrenzen in Abhängigkeit der Knochendichtemessung. Dies findet in den Therapiempfehlungen des FRAX-Scores keine Berücksichtigung. Mutmaßlich zeigte sich im untersuchten Kollektiv eine solch markanter Anstieg der Therapiegrenzen unter Einbeziehung der Knochendichtemessung, da im vorselektionierten Kollektiv des MVZ endokrinologikum Göttingen eine im Vergleich zu anderen Kollektiven sehr geringe durchschnittliche Knochendichtemessung vorlag (minimaler T-Score am Femurhals 2,1 $\pm 1,0$, minimaler T-Score insgesamt $-2,8 \pm 0,9)$. Um die Genauigkeit der ermittelten Therapiegrenzen zu überprüfen, sollte man diese in einem Vergleichskollektiv überprüfen.

Tabelle 29: Therapiegrenzen FRAX-Score basierend auf den Daten des MVZ endokrinologikum in Anlehnung an Tabelle 17

\begin{tabular}{|c|c|c|c|c|c|c|c|}
\hline & $\begin{array}{l}\text { Gemittelte } \\
\text { Therapiegrenze }\end{array}$ & Standardabweichung & Minimum & Maximum & Median \\
\hline \multirow{4}{*}{ 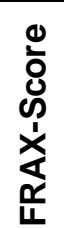 } & \multirow{2}{*}{$\begin{array}{l}\text { Ohne } \\
\text { BMD }\end{array}$} & MOF & 6,92 & 3,81 & 2,00 & 20,0 & 6 \\
\hline & & HF & 2,47 & 2,8 & 0,1 & 15,0 & 1,6 \\
\hline & \multirow{2}{*}{$\begin{array}{l}\text { Mit } \\
\text { BMD }\end{array}$} & MOF & 14,43 & 12,99 & 2,20 & 85,0 & 11 \\
\hline & & HF & 9,18 & 12,85 & 0,1 & 84,0 & 5,7 \\
\hline
\end{tabular}

Der Score nach C. Glüer unterschied sich nicht signifikant vom FRAX- bzw. „FRAX+“-Scores für eine Hüftfraktur ohne Einbeziehung der Knochendichtemessung. Auch der FRAX- bzw. „FRAX+“-Score für eine „major osteoporotic“ Fraktur mit Knochendichtemessung unterschied sich nicht signifikant vom Score nach C. Glüer. Diese Beobachtung war unabhängig der gewählten Therapiegrenze von $\geq 14 \%$ bzw. $\geq 20 \%$. Beide Scores zeigten eine Gewichtung der Risikofaktoren für die Berechnung des Frakturrisikos. Beim Score nach C. Glüer lag eine 
Einteilung der Risikofaktoren in moderate, starke und sehr starke vor (vergleiche Tabelle 10). Für den FRAX-Score gab es keine offiziellen Angaben, in welcher Wichtung die einzelnen Faktoren in den Algorithmus einflossen und wie sie interagierten. Da sich in dem in dieser Arbeit untersuchten männlichen Patientenkollektiv jedoch keine signifikanten Unterschiede in den Therapieentscheidungen zwischen dem FRAX- bzw. „FRAX+“-Score für eine Hüftfraktur ohne Einbeziehung der Knochendichtemessung bzw. für eine „major osteoporotic“ Fraktur mit Einbeziehung der Knochendichtemessung und dem Score nach C. Glüer zeigte, ließe sich schlussfolgern, dass die Wichtungen der Risikofaktoren in Kombination mit den für den jeweiligen Score gewählten Therapiegrenzen sich in Bezug auf die daraus resultierenden Therapieempfehlungen ähnelten.

Basierend auf dem WHO-Kriterium von einem minimalem T-Score $\leq-2,5$ (gemessen am Femurhals, Gesamtfemur oder der LWS) zur Definition einer Osteoporose lag für $63,84 \%$ der untersuchten Patienten eine positive Therapieempfehlung vor. Unerwartet wurden Patientenkollektiv des MVZ endokrinologikum Göttingen durch keinen der untersuchen Scores eine höhere Zahl an Therapieempfehlungen ausgesprochen. Bezüglich der Therapieempfehlungen basierend auf den WHO-Kriterien zeigte sich eine signifikante Übereinstimmung mit dem DVOScore, sowie dem FRAX-Score für eine Hüftfraktur unter Einbeziehung der Knochendichtemessung. Dieser besonders hohe Anteil an positiven Therapieempfehlungen, basierend auf den WHO-Kriterien, lässt sich durch den sehr geringen durchschnittlichen minimalen T-Score von $-2.8 \pm 0,9$ im Patientenkollektiv des MVZ endokrinologikum Göttingen erklären. Für eine Therapieentscheidung allein basierend auf der Knochendichte liegt laut Studien eine hohe Sensitivität vor. Die Spezifität ist jedoch gering (Johansson et al. 2017; WHO 1991). Es zeigte sich, dass der Einfluss der Knochendichte an der Prognose des Frakturrisikos mit dem Alter abnimmt. Ein T-Score $\leq 2,5$ gilt so in jungen Jahren als starker Risikofaktor. Bedingt durch die pathophysiologische Abnahme der Knochendichte sowie weitere frakturbegünstigende Faktoren wie beispielweise eine Abnahme der Muskelmasse, eine erhöhte Sturzneigung und weitere osteoporosebegünstigende Komorbiditäten wird einem entsprechendem T-Score im höheren Alter eine andere Gewichtung als Risikofaktor beigemessen (Johansson et al. 2017; Kanis et al. 2016b). Basierend auf dem niedrigem Durchschnittalter der Patienten im MVZ endokrinologikum Göttingen, dem hohen prozentualen Anteil an osteoporosebegünstigenden Vorerkrankungen und den möglicherweise daraus resultierenden niedrigen T-Scores ergab sich durch den Einschluss weiterer Risikofaktoren kein prozentualer Zugewinn an Therapieentscheidungen im Vergleich zu einer Therapieschwelle orientiert an einem T-Score $\leq-2,5$. Auch in unserem Kollektiv zeigte sich somit, dass der T-Score einen von weiteren Risikofaktoren unabhängig Einfluss auf das 10-Jahres-Frakturrisiko hat. 
Epidemiologische Studien zeigten keine Verbesserung der 10-Jahres-Frakturprognose durch die Verwendung des minimalen T-Scores (gemessen am Femurhals, Gesamtfemur oder der LWS) im Vergleich zu Kollektiven, die nur den T-Score am Femurhals berücksichtigten. Sie zeigten unter Integration aller Messorte sogar eine Überschätzung des Frakturrisikos im Vergleich zu den tatsächlich aufgetretenen Frakturen (Kanis et al. 2006; Leslie et al. 2007). Zudem belegen Studien eine Verbesserung der Frakturprognose durch die zusätzliche Einbeziehung von Risikofaktoren im Vergleich zu Scores, die sich nur auf die Knochendichtemessung beziehen (Leslie und Lix 2011). Basierend auf den vorliegenden Daten des MVZ endokrinologikum Göttingen ließe sich schlussfolgern, dass sowohl anhand der WHO-Kriterien, als auch basierend auf dem DVO-Score das tatsächliche Frakturrisiko überschätzt wird, da hier jeweils der minimale T-Score (gemessen am Femurhals, Gesamtfemur oder der LWS) verwendet wurde. Letztlich haben wir in dem hier vorliegenden Kollektiv nur die Scores verglichen. Nur prospektive Studien können abschließend nachweisen welcher der Scores am zutreffensten das 10Jahres-Frakturrisiko in diesem stark vorselektionierten Kollektiv abbildet.

\subsection{Limitationen und Ausblick}

Bei der im Rahmen dieser Arbeit untersuchten Daten aus dem MVZ endokrinologikum Göttingen handelt es sich um eine retrospektive Studie. Im Vergleich zu anderen Studien war folglich keine Aussage über die Güte der Frakturprognose möglich. Um eine Aussage diesbezüglich treffen zu können, wäre eine erneute Patientenbefragung zur Erhebung der im Vergleich zur Erstvorstellung stattgehabten Frakturen notwendig. Somit ließe sich auch überprüfen, ob die zusätzliche Verwendung von sekundären Osteoporoseursachen im „FRAX+“-Modell zu einer Verbesserung der 10-Jahres-Frakturporgnose bzw. Therapieempfehlungen geführt hat. Dabei gilt es zu berücksichtigen, dass ein Großteil der Patienten, basierend auf dem über 30\% Frakturrisiko vermutlich eine antiosteoporotische Therapie erhalten hat. Dies müsste in der Auswertung berücksichtigt werden, da durch eine adäquate Therapie eine signifikante Frakturreduktion zu erwarten ist. Da es sich hier um Daten aus dem Zeitraum vom 01.07.2007 30.06.2014 handelt, wäre bereits für einen Großteil der Patienten eine Auswertung der in den letzten 10 Jahren stattgehabten Frakturen möglich. Für den Score nach C. Glüer, der sich auf das 1-Jahres-Frakturrisiko bezieht, wäre eine solche Patientenbefragung zur Erfassung der tatsächlich stattgehabten Frakturen ebenfalls gut durchzuführen.

Weiterhin gilt es zu beachten, dass lediglich der FRAX-Score an einem ausreichend großem Patientenkollektiv validiert ist. Der DVO-Score ist anhand eine weiblichen Patientenkollektivs der „FREEDOM“-Studie validiert, der Score nach C. Glüer und der „FRAX+“-Score sind dahingegen gar nicht validiert. Zudem wurde der Score nach C. Glüer, der sich auf ein 1-JahresFrakturrisiko bezieht zur besseren Vergleichbarkeit mit dem Faktor 10 multipliziert, um so ein 
näherungsweises 10-Jahres-Frakturrisiko zu erhalten. Somit ist mit einer Überschätzung der Frakturrisiken durch die fehlende Einkalkulierung der Mortalität zu rechnen.

Eine weitere Limitation der Arbeit stellt die geringe Anzahl an männlichen Vergleichskohorten da. Die wenigen vorliegenden Vergleichskollektive zeigen zudem eine starke Abweichung bezüglich der Einschlusskriterien. Sie stellen epidemiologische Studien dar, wohingegen die Daten aus dem MVZ endokrinologikum Göttingen ein stark vorselektionieres Patientengut einschließt. So zeigte sich im Vergleich ein deutlich höheres Risikoprofil der Patienten des MVZ endokrinologikum Göttingen, bei zugleich niedrigerem durchschnittlichem Alter und geringeren T-Scores. Das vorliegende männliche Patientenkollektiv gibt somit kein Bild der altersentsprechenden Normalbevölkerung wider.

Ein weiterer Punkt, den es zu berücksichtigen gilt ist, dass die Empfehlung des DVO Scores für männliche Patienten ab einem Alter initial von 50 Jahren gelten. Im hier vorliegenden Studienkollektiv lag das Durchschnittsalter bei 58,9 Jahren, 17,0\% der untersuchten Patienten waren unter 60 Jahren.

\subsection{Zusammenfassung}

Die frühe Krankheitsperiode der Osteoporose verläuft oftmals schmerzlos. Bis zu $80 \%$ der ostoporoseassoziierten Frakturen sind klinisch inapparent. Nach stattgehabter osteoporotischer Fraktur zeigt sich bei Männern eine signifikant erhöhte Mortalität im Vergleich zu Frauen. Hinzu kommt, dass das Bewusstsein für eine osteoporotische Erkrankung bei Männern weitaus weniger präsent ist, als bei Frauen (Misiorowski 2017). Diese Fakten verdeutlichen die hohe Relevanz eines einfachen, zuverlässigen Tools zur Ermittlung des osteoporoseassoziierten Frakturrisikos.

In der hier vorliegenden Arbeit wurden in einer retrospektiven Studie Patientendaten von 147 männlichen Patienten aus dem Drittzuweiserzentrum MVZ endokrinologikum Göttingen bezüglich der osteoporoseassoziierten 10-Jahres-Frakturwahrscheinlichkeit und der sich daraus ergebenen Therapieempfehlungen ausgewertet. Es handelte sich dabei um ein Patientenkollektiv, das zwar im Vergleich mit anderen Kollektiven durchschnittlicher jünger war (Durchschnittsalter 58,9 Jahre), sich jedoch durch ein höheres Risikoprofil und deutlich niedrigere TScores (durchschnittlicher minimaler T-Score -2,8) auszeichnete. Es wurden bereits validierte Scores, wie der FRAX-Score und DVO-Score, sowie nicht validierte Scores, wie der „FRAX+“Score und Score nach C. Glüer untersucht. Sie unterschieden sich sowohl im Set der gewählten Risikofaktoren als auch in den Therapiegrenzen. Es zeigte sich, dass die durch den FRAXScore berechneten 10-Jahres-Frakturrisiken sich hoch signifikant von den DVO-basierten Frakturrisiken unterschieden. Für das untersuchte Patientenkollektiv zeigte sich ferner keine Veränderung der 10-Jahres-Frakturprognosen unter Einbeziehung zusätzlicher sekundärer 
Osteoporseformen in Abhängigkeit von der Knochendichtemessung. Bezüglich der Therapieentscheidungen zeigte sich nur zwischen dem DVO-Score und dem FRAX- bzw. „FRAX+“Score für eine Hüftfraktur mit Einbeziehung der Knochendichtemessung eine hoch signifikante Übereinstimmung. Alle anderen FRAX-Scores unterschieden sich hoch signifikant vom DVOScore.

Legt man anhand der durchgeführten Auswertungen der Daten des MVZ endokrinologikum Göttingen neue Therapiegrenzen für den FRAX-Score fest, so zeigten sich teils markante Abweichungen von den in Metaanalysen festgelegten Therapiegrenzen von $\geq 14 \%$ bzw. $\geq 20 \%$ für eine „major osteoporotic“ Fraktur bzw. $\geq 3 \%$ für eine Hüftfraktur (Kanis et al. 2016a). Sie lagen im untersuchten männlichen Patientenkollektiv ohne Einbeziehung der Knochendichte bei $\geq 6,92( \pm 3,81)$ für eine „major osteoporotic“ Fraktur bzw. bei $\geq 2,47( \pm 2,8)$ für eine Hüftfraktur. Unter Einbeziehung der Knochendichtemessung ergaben sich abweichende Therapiegrenzen. Sie lagen bei $\geq 14,43( \pm 12,99)$ für eine "major osteoporotic“ Fraktur bzw. $\geq 9,18$ $( \pm 12,85)$ für ine Hüftfraktur.

Im Vergleich zum einfachen WHO-basierten Therapieentscheidungstool, das sich lediglich an einem T-Score $\leq-2,5$ orientiert, zeigte sich im hier untersuchten, stark vorselektionierten Patientenkollektiv kein prozentualer Zugewinn an Therapieempfehlungen (Kanis et al. 1994; WHO 1991). Die meisten Patienten (63,84\%) erhielten eine Therapieempfehlungen basierend auf diesem einfachen Therapieentscheidungstool. Durch den DVO-Score und FRAX-Score für eine Hüftfraktur unter Einbeziehung der Knochendichte wurde zwar prozentuale weniger Patienten eine Therapie empfohlen, dieser Unterscheid war jedoch nicht hochsignifikant. In einer weiteren Arbeit gilt es zu überprüfen, inwiefern die durch die verschiedenen Scores errechneten 10-Jahres-Frakturrisiken in dem untersuchten Kollektiv an männlichen Patienten mit den tatsächlich aufgetreten Frakturen übereinstimmen. 


\section{Anhang}

\subsection{Modifizierung der Therapiegrenze nach den DVO-Leitlinien 2014 (DVO Leitlinien 2014; mit freundlicher Genehmigung durch den DVO)}

\begin{tabular}{|c|c|}
\hline $\begin{array}{l}\text { Indikation zur Basisdiagnostik anhand der DVO-Leitlinie } 2014 \text { zur Pro- } \\
\text { phylaxe, Diagnostik und Therapie der Osteoporose bei postmenopausa- } \\
\text { len Frauen und bei Männern ab dem 60. Lebensjahr }\end{array}$ & $\begin{array}{l}\text { Wert, um den die Therapiegrenze } \\
\text { ansteigt }\end{array}$ \\
\hline Niedrigtraumatische singuläre Wirbelkörperfraktur 2. - 3. Grades *2) & ${ }^{*}$ Ausnahmeregelung \\
\hline $\begin{array}{l}\text { Niedrigtraumatische singuläre klinisch manifeste Wirbelkörperfraktur } 1 . \\
\text { Grades mit Deckplattenimpression*2) }\end{array}$ & $+0,5$ \\
\hline $\begin{array}{l}\text { Niedrigtraumatische singuläre Wirbelkörperfraktur 1. Grades mit Deck- } \\
\text { platten-impression ohne Klinik als Einzelfallentscheidung }{ }^{* 1,2)}\end{array}$ & $+0,5$ \\
\hline Niedrigtraumatische multiple Wirbelkörperfrakturen 1.-3.Grades *2) & * Ausnahmeregelung \\
\hline $\begin{array}{l}\text { Niedrigtraumatische nicht-vertebrale Frakturen mit Ausnahme von Fin- } \\
\text { ger-, Zehen-, Schädel- u. Knöchelfrakturen }\end{array}$ & $\begin{array}{l}+1 \text { (wenn > } 3 \text { Frakturen in den letz- } \\
\text { ten } 10 \text { Jahren), ansonsten - } 0,5 \\
\text { (wenn nach dem 50. Lebensjahr) }\end{array}$ \\
\hline Cushing Syndrom oder subklinischer Hyperkortisolismus*4) & $+0,5$ \\
\hline Primärer Hyperparathyreoidismus $\left.{ }^{4}\right)$ & $+0,5$ \\
\hline Wachstumshormonmangel *4) & 0 \\
\hline $\begin{array}{l}\text { Bestehende oder geplante orale Glukokortikoidtherapie mit } \geq 2,5 \mathrm{mg} \\
\text { Prednisolonäquivalent tgl. für mehr als } 3 \text { Monate im Jahr *4) }\end{array}$ & +1 \\
\hline Epilepsie / Antiepileptika *4) & $+0,5$ \\
\hline Monoklonale Gammopathie unklarer Signifikanz & 0 \\
\hline Rheumatoide Arthritis & $+0,5$ \\
\hline Diabetes mellitus Typ 1 & +1 \\
\hline Zustand nach B-II-Operation oder Gastrektomie & 0 \\
\hline Spondylitis ankylosans als Einzelfallentscheidung *1) & $+0,5$ \\
\hline Therapie mit Aromatasehemmern als Einzelfallentscheidung *1)4) & $+0,5$ \\
\hline Therapie mit Aromatasehemmern *3)4) & $+0,5$ \\
\hline Glukokortikoide hochdosiert inhalativ *3)4) & $+0,5$ \\
\hline $\begin{array}{l}\text { Hormonablative Therapie oder Hypogonadismus beim Mann als Einzel- } \\
\text { fallentscheidung *1/4) }\end{array}$ & $+0,5$ \\
\hline Hormonablative Therapie oder Hypogonadismus beim Mann *3) 4) & $+0,5$ \\
\hline Therapie mit Glitazonen *3)4) & $+0,5$ \\
\hline Diabetes mellitus Typ 2 *3) & 0 \\
\hline Spondylitis ankylosans *3) & $+0,5$ \\
\hline Proximale Femurfraktur eines Elternteils *3) & $+0,5$ \\
\hline
\end{tabular}


6 Anhang

\begin{tabular}{|l|l|}
\hline Multiple intrinsische Stürze oder erhöhte Sturzneigung *3)4) & $+0,5$ \\
\hline Immobilität *3)4) & $+0,5$ \\
\hline Untergewicht $\left.\left.\left(\mathrm{BMI}<20 \mathrm{~kg} / \mathrm{m}^{2}\right)^{\star 3}\right)^{4}\right)$ & 0 \\
\hline Nikotinkonsum und / oder COPD *3)4) & $+0,5$ \\
\hline Depression / Antidepressiva *3)4) & $+0,5$ \\
\hline $\begin{array}{l}\text { Hyperthyreose oder subklinische Hyperthyreose, sofern persistierend *3) } \\
4)\end{array}$ & $+0,5$ \\
\hline Zöliakie *3) & $+0,5$ \\
\hline Protonenpumpeninhibitoren bei chronischer Einnahme *3)4) & $+0,5$ \\
\hline Herzinsuffizienz *3)4) & $+0,5$ \\
\hline
\end{tabular}


6.2 Patientenfragebogen (mit freundlicher Genehmigung durch H. Siggelkow)

Name:

endokrinologikum

Datum:

\author{
Ärztliche Fragen vor der Untersuchung \\ Mit welchen Beschwerden kommen Sie zu uns? \\ (alle Ihre Angaben unterliegen der ärztlichen Schweigepflicht)
}

Größe: Gewicht:

Beantworten Sie bitte die folgenden Fragen durch Ankreuzen der mit "Ja" oder "Nein" bezeichneten Kăstchen.

Haben Sie zur Zeit:

Kopf- oder Gesichtsschmerzen

Nackenschmerzen

Sehstörungen, Doppelbilder

Hörstörungen, Ohrensausen

Riech- oder Geschmacksstörungen

Schwindelgefühl

starken Haarausfall

Atemnot

Plötzliches Herzjagen

Herzstolpern

Appetitlosigkeit

Gewichtsabnahme

Leibschmerzen

Durchfall

Verstopfung

Năchtliches Wasserlassen

Wenn ja, wie oft

Rückenschmerzen

Ischiasschmerzen

Kribbeln, Ameisenlaufen,

Taubheitsgefühl in

Armen, Beinen oder im Gesicht

Schwellungen (Gesicht, Arme, Beine)

Venenentzündungen

Häufige blaue Flecke

Langes Bluten nach Verletzungen

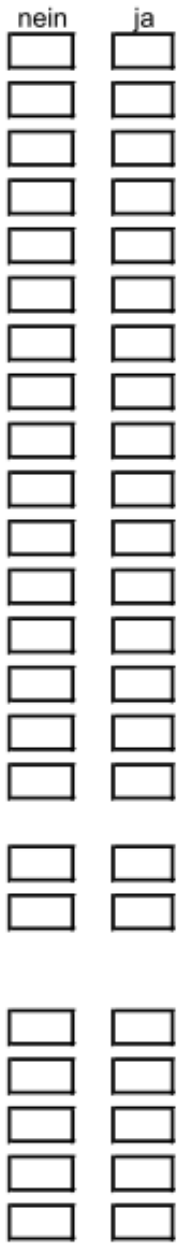


Drūsenschwellungen

Schlafstörungen

Leichte Erschöpfbarkeit

Konzentrations- und Gedāchtnisstörung

Vermehrtes Schwitzen

Vermehrter Durst

Wieviel Liter trinken Sie pro Tag:

Fühlten Sie sich im letzten Monat hăufig niedergeschlagen,

traurig, bedrückt oder hoffnungslos?

Hatten Sie im lezten Monat deutlich weniger Lust und

Freude an Dingen, die Sie sonst gern tu

\section{Genussmittel (bitte genau angeben)}

1. Alkoholische Getrănke (z. B. Bier, Wein,

Was trinken Sie und wieviel pro Tag?

2. Rauchen (z. B. Zigaretten, Zigarren,

Pfeife)

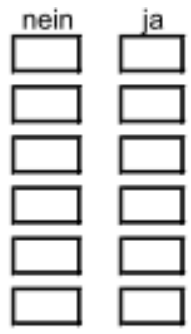

Was rauchen sie und wieviel pro Tag?

3. Andere Genußmittel (z. B. Kaffee, Te

\section{Medikamente (bitte genau angeben)}

Nehmen Sie regelmaßßig Medikamente $\mathbf{~}$

Welche?

Gelegentliche Einnahme?

Welche?

Sind Sie überempfindlich gegen bestimmte

Speisen, Medikamente oder anderes?

(bitte ankreuzen) 
Haben Sie früher einmal eine der folgenden Krankheiten gehabt

Gelbsucht (Hepatitis)

Hirnhautentzündung

Kinderlăhmung

Geschlechtskrankheit

Tuberkulose

Andere ansteckende Krankheiten

Gelenkkrankheiten, Rheuma

Nieren- und Blasenkrankheiten

Nierensteine

Herzerkrankungen

Bluthochdruck festgestellt seit wann

Lungenerkrankungen

Magenerkrankungen

Lebererkrankungen

Gallenblasenerkrankungen

Bauchspeicheldrüsenerkrankungen

Darmerkrankungen

Zuckerkrankheit festgestellt

Schilddrūsenerkrankungen

Hormonstörungen

Bestrahlungen

Wenn ja, wann?

Unfallverletzungen

Gehirnerschütterung, Schädelbruch
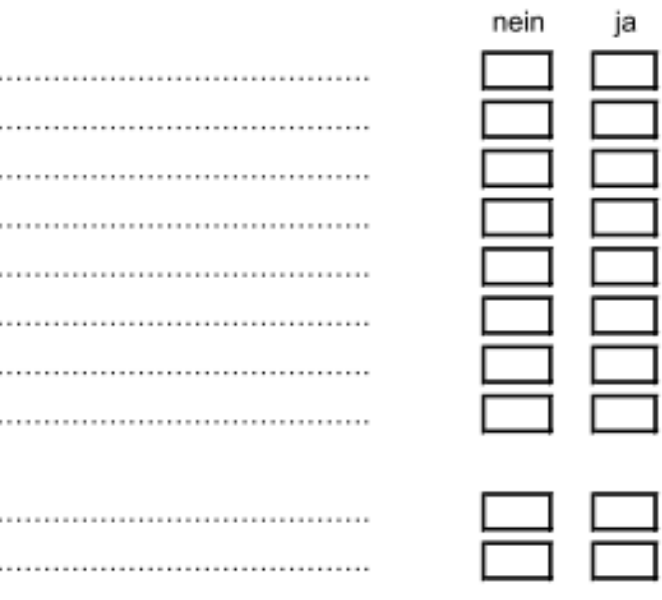

Operationen

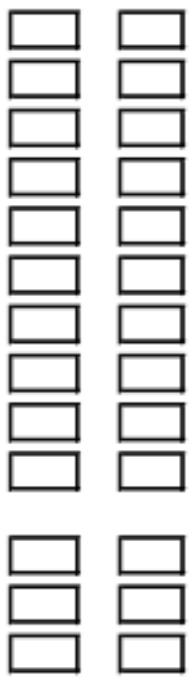

1.:

$2 .:$

3.

Haben Sie eine Pflegestufe?

wenn ja, welche

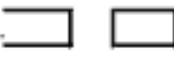

Haben Sie eine Pflegestufe beantragt?

Sind Sie schwerbehindert und haben einen Schwerbehindertenausweis?

Welchen Beruf üben Sie augenblicklich 
Für Frauen

(Zutreffendes bitte ankreuzen)

nein ja

Erste Monatsblutung mit Jahren

Letzte Monatsblutung am

(Datum/Jahr)

Haben Sie Regelstörungen?

Hatten Sie Geburten?

Wieviel und in welchem Jahr?

Wie lange haben Sie insgesamt gestillt?

(in Monaten)

Nehmen Sie z. Zt. Eine Antibabypille?

Wenn ja, wie lange?

Wurde Gebärmutter entfernt?

Wurden Eierstōcke entfernt?

\section{Familienvorgeschichte}

(Eltern, Großeltem, Geschwister der Eltern, eigene Geschwister, eigene Kinder, nicht Sie selbst) Hat oder hatte jemand in Ihrer Familie eine der folgenden Krankheiten:

\section{Zuckerkrankheit}

Hoher Blutdruck

Herzinfarkt

Schlaganfall

nein ja wer?

Asthma

Krebs

Fettleibigkeit

Rundrücken oder Osteoporose

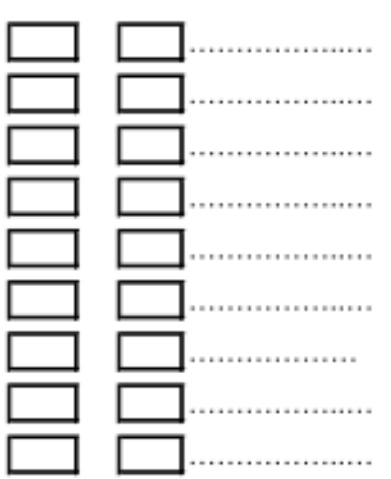

Knochenbrüche 


\subsection{Osteoporose-Risikofragebogen für Patienten (mit freundlicher Genehmigung durch} H. Siggelkow)

\section{endokrinologikum \\ Osteoporose-Risikofragebogen für Patienten \\ anhand der Empfehlung des Dachverbandes der osteologischen Fachgesellschaften DVO}

Liebe Patientinnen, liebe Patienten,

die folgenden Fragen sollen helfen, Ihre Knochen-Gesundheit zu ermitteln. Bitte beantworten Sie deshalb die folgenden Fragen, die zur Beurteilung Ihres Osteoporose-Risikos dienen. Alle Informationen unterliegen der ärztlichen Schweigepflicht. Bitte kreuzen Sie nur an, was zutrifft.

1. Ist bei Ihnen eine Osteoporose bekannt

2. Regelblutung bei Frauen

Wie alt waren Sie bei der 1. Regel?

b Wie alt waren Sie bei der letzten Regelblutung

3. Gibt es Osteoporose in Ihrer Familie: Hat/Hatte Ihre Großmutter/Mutter/Schwester/ Bruder eine Osteoporose, einen krummen Rücken/einen Unterarm-, Wirbel- oder Schenkelhalsbruch? (Bitte zutreffendes unterstreichen)

4. Leiden Sie an einer der folgenden Erkrankungen:

a an einer Schilddrũsenerkrankung, wegen der Sie Tabletten einnehmen?

b an chronischem Durchfall? an einem entzūndlichen Gelenkrheumatismus?

c an einem Anfallsleiden / Epilepsie, nehmen

d Sie deswegen Tabletten?

5. Medikamentenanamnese: Namen Sie über lăngere Zeit folgende Medikamente ein:

Cortison

Schlafmittel

Marcumar/ Sintrom

Schilddrûsenhormone

Wenn ja, wie lange?

Nahmen Sie bereits folgende Medikamente ein?

\section{Calcitonin}

Calcium

Fluor

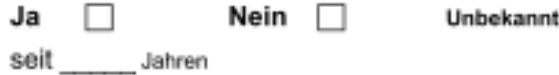

Jahre

Jahre

Ja

Nein

Unbekannt

Wenn ja, wie lange?

$\begin{array}{llll}\text { Ja } & \square & \text { Nein } \square & \text { Unbekannt } \square \\ \text { Ja } & \square & \text { Nein } \square & \text { Unbekannt } \square \\ \text { Ja } & \text { Nein } \square & \text { Unbekannt } \square \\ \text { Ja } & \square & \text { Nein } \square & \text { Unbekannt }\end{array}$

6. Haben Sie Rūckenschmerzen?

beim Tragen oder Heben

beim längeren Stehen

beim Sitzen

beim Laufen

Sind diese Rückenschmerzen neu,

haben sie sich akut verschlechtert?

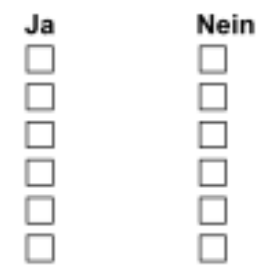


Osteoporose-Risikofragebogen für Patienten anhand der Empfehlung des Dachverbandes der osteologischen Fachgesellschaften DVO

7 - 9 Lebensgewohnheiten:

\begin{tabular}{|l|c|c|c|c|}
\hline Zigarettenkonsum: & keinen & unter 10 Zig. & bis 20 Zig. & uber 20 Zig. \\
\hline Alkoholkonsum: & keinen & gelegentlich & bis 2/4 Wein & über 2/4 Wein \\
\hline Diat: & keinen & (fast) vegetarisch & Diabetes & sonstiges \\
\hline Milchkonsum: & keinen & wenig & bis 1/4 tgl. & mehr \\
\hline Käse u. Joghurt: & Keinen & manchmal & öfters & viel \\
\hline Sport: & keinen & fallweise & regelmaßig & viel \\
\hline Immobilităt: & nie & 1 Monat & 2 Monate & mehr \\
\hline
\end{tabular}

10 Ihre Körpergröße

a Was war lhre max. Körpergröße? etwa?

11 Ihr Körpergewicht

a Was war Ihr höchstes Gewicht? Im Alter von

b Haben Sie in den letzten 2 Jahren an Gewicht abgenommen?

12 Wie aktiv sind Sie körperlich?

a z. B. verlassen Sie (fast) tãglich länger als 1 Stunde thre Wohnung zum Einkaufen oder Wandern, Radtahren, Gertenarbeit usw.

b Machen Sie Gymnastik, Sport u.a.m.? Wie of / Woche:

13 Ist thre Beweglichkeit eingeschränkt?

a Sind sie gehbehindert?

b Benutzen Sie eine Gehhilfe?

c Haben Sie Probleme mit Ihren Füßen?

14 Fragen zu Ihrem Sturzrisiko

a Sind Sie innerhalb des letzten Jahres gestürzt?

b Leiden Sie an Bluthochdruck?

c Leiden Sie an Schwindel?

d Hatten Sie einen Schlaganfall?

e Nehmen Sie Schlaf- oder Beruhigungsmittel ein?

f Sehen Sie schlecht? Z.B. sehr starke Brile, Stabsehen, einalugig

15 Fragen zu Knochenbrüchen

a Hatten Sie seit dem 50. Lebensjahr einen Knochenbruch? Ja

b Wodurch trat dieser Knochenbruch auf?

im Alltagsieben (z.B. beim Heben, Tragen, Laufen, beim Lagewechesi

Ausnutschen, Stolpern U. a)

durch Sturz z B. won der Leiter, vom Stuhl, auf der Treppe, vom Baum durch schweren Sturz

durch Unfall (z. B. Verkehrsunfall) durch einen Unfall

Mit der Erfassung dieser Daten in der Ambulanzdokumentation bin ich einverstanden

Datum und Unterschrift: 


\section{Literaturverzeichnis}

Aggarwal L, Masuda C (2018): Osteoporosis: A quick update. J Fam Pract $\underline{67}, 59-65$

Allolio B, Schulte H. (Hrsg.): Praktische Endokrinologie. 2. Auflage; Elsevier, München 2010

Amin S, Zhang Y, Felson DT, Sawin CT, Hannan MT, Wilson PW, Kiel DP (2006): Estradiol, testosterone, and the risk for hip fractures in elderly men from the Framingham Study. Am J Med 119, 426-433

Aspray TJ (2015): Fragility fracture: recent developments in risk assessment. Ther Adv Musculoskelet Dis $\underline{7}, 17-25$

Backhaus K, Erichson B, Plinke W, Weiber R: Multivariate Analysemethoden: Eine Anwendungsorientierte Einführung. 13. Auflage, Springer-Verlag, Berlin 2013

Bartl C, Bartl R (2011): Secondary osteoporosis: pathogenesis, types, diagnostics and therapy. Radiologe 51, 307-324

Bolland MJ, Siu AT, Mason BH, Horne AM, Ames RW, Grey AB, Gamble GD, Reid IR (2011): Evaluation of the FRAX and Garvan fracture risk calculators in older women. J Bone Miner Res $\underline{26}$, 420-427

Brandi ML (2009): Microarchitecture, the key to bone quality. Rheumatology $\underline{48}$, iv3-iv8

Cauley JA (2011): Defining ethnic and racial differences in osteoporosis and fragility fractures. Clin Orthop Relat Res $\underline{469}$, 1891-1899

Chen H, Zhou X, Fujita H, Onozuka M, Kubo K-Y (2013): Age-Related Changes in Trabecular and Cortical Bone Microstructure. Int J Endocrinol 2013, 9,213234

De Laet C, Kanis JA, Oden A, Johanson H, Johnell O, Delmas P, Eisman JA, Kroger H, Fujiwara S, Garnero P, et al. (2005): Body mass index as a predictor of fracture risk: a meta-analysis. Osteoporos Int $\underline{16}, 1330-1338$

DVO-Leitlinie 2009 zur Prophylaxe, Diagnostik und Therapie der Osteoporose im Erwachsenenalter. Schattauer, o.O. 2009

DVO-Leitlinie 2014 zur Prophylaxe, Diagnostik und Therapie der Osteoporose bei Männern ab dem 60 Lebensjahr und bei postmenopausalen Frauen. Schattauer, o.O. 2014

DVO-Leitlinie 2017 zur Prophylaxe, Diagnostik und Therapie der Osteoporose bei postmenopausalen Frauen und Männern. Schattauer, o.O. 2018

DVO-Leitlinienreport 2017 zur S3-Leitlinie zur Prophylaxe, Diagnostik und Therapie der Osteoporose bei postmenopausalen Frauen und Männern. Schattauer, o.O. 2018

El Maghraoui A, Roux C (2008): DXA scanning in clinical practice. QJM 101, 605-617

El Maghraoui A, Mouinga Abayi DA, Ghozlani I, Mounach A, Nouijai A, Ghazi M, Achemlal L, Bezza A (2007): Prevalence and risk factors of discordance in diagnosis of osteoporosis using spine and hip bone densitometry. Ann Rheum Dis $\underline{66}, 271-272$

Ensrud KE, Lui LY, Taylor BC, Schousboe JT, Donaldson MG, Fink HA, Cauley JA, Hillier TA, Browner WS, Cummings SR (2009): A comparison of prediction models for fractures in older women: is more better? Arch Intern Med $\underline{169}$, 2087-2094 
Espallargues M, Sampietro-Colom L, Estrada MD, Solà M, del Río L, Setoain J, Granados A (2001): Identifying Bone-Mass-Related Risk Factors for Fracture to Guide Bone Densitometry Measurements: A Systematic Review of the Literature. Osteoporos Int 12, 811-822

Ettinger B, Ensrud KE, Blackwell T, Curtis JR, Lapidus JA, Orwoll ES (2013): Performance of FRAX in a cohort of community-dwelling, ambulatory older men: The Osteoporotic Fractures in Men (MrOS) study. Osteoporos Int 24, 1185-1193

Fink HA, Milavetz DL, Palermo L, Nevitt MC, Cauley JA, Genant HK, Black DM, Ensrud KE, Fracture Intervention Trial Research G (2005): What proportion of incident radiographic vertebral deformities is clinically diagnosed and vice versa? J Bone Miner Res $\underline{20}, 1216-1222$

Flais J, Coiffier G, Le Noach J, Albert JD, Faccin M, Perdriger A, Thomazeau H, Guggenbuhl P (2017): Low prevalence of osteoporosis treatment in patients with recurrent major osteoporotic fracture. Arch Osteoporos $\underline{12}, 24$

Forsen L, Sogaard AJ, Meyer HE, Edna T, Kopjar B (1999): Survival after hip fracture: short- and long-term excess mortality according to age and gender. Osteoporos Int 10, 7378

FRAX-Eingabemaske, https://www.shef.ac.uk/FRAX/tool.jsp?lang=de9, Zugriff am: 18.02.2016

Garvan-Eingabemaske,http://www.garvan.org.au/promotions/bone-fracturerisk/calculator/, Zugriff am: 02.11.2017

Gennari L, Nuti R, Bilezikian JP (2004): Aromatase activity and bone homeostasis in men. J Clin Endocrinol Metab 89, 5898-5907

Gluer CC (2013): DVO-Leitlinien 2013 - Modellierung des Frakturrisikos. Kongress Osteologie 2013. Weimara 07.09.03.2013 i

Gluer CC, Eastell R, Reid DM, Felsenberg D, Roux C, Barkmann R, Timm W, Blenk T, Armbrecht G, Stewart A, et al. (2004): Association of five quantitative ultrasound devices and bone densitometry with osteoporotic vertebral fractures in a population-based sample: the OPUS Study. J Bone Miner Res $\underline{19}$, 782-793

Gourlay ML, Ritter VS, Fine JP, Overman RA, Schousboe JT, Cawthon PM, Orwoll ES, Nguyen TV, Lane NE, Cummings SR, et al. (2017): Comparison of fracture risk assessment tools in older men without prior hip or spine fracture: the MrOS study. Arch Osteoporos 12, 91

Hadji P, Bock K, Emons G, Wüster C, Schulz K (2002): Früherkennung und Prävention der Osteoporose. Gynäkologe $\underline{35}, 518-526$

Hadji P, Bock O, Resch H, Kraenzlin M, Wang A, Steinle T, Eisen C (2013a): Reduktion des Frakturrisikos unter Denosumab bei postmenopausalen Frauen in Abhängigkeit der Behandlungsbedürftigkeit nach DVO-Leitlinien 2009. Osteologie 1, 39-45

Hadji P, Klein S, Gothe H, Haussler B, Kless T, Schmidt T, Steinle T, Verheyen F, Linder R (2013b): The epidemiology of osteoporosis--Bone Evaluation Study (BEST): an analysis of routine health insurance data. Dtsch Arztebl Int 110, 52-57

Hannan MT, Felson DT, Dawson-Hughes B, Tucker KL, Cupples LA, Wilson PW, Kiel DP (2000): Risk factors for longitudinal bone loss in elderly men and women: the Framingham Osteoporosis Study. J Bone Miner Res $\underline{15}$, 710-720 
Hans D, Downs RW, Jr., Duboeuf F, Greenspan S, Jankowski LG, Kiebzak GM, Petak SM, International Society for Clinical D (2006): Skeletal sites for osteoporosis diagnosis: the 2005 ISCD Official Positions. J Clin Densitom $\underline{9}$, 15-21

Harvey NC, Oden A, Orwoll E, Lapidus J, Kwok T, Karlsson MK, Rosengren BE, Ljunggren O, Cooper C, McCloskey E, et al. (2017): Falls Predict Fractures Independently of FRAX Probability: A Meta-Analysis of the Osteoporotic Fractures in Men (MrOS) Study. J Bone Miner Res $\underline{33}, 510-516$

Häussler B, Gothe H, Mangiapane S, Glaeske G, Pientka L, Felsenberg F (2006): Versorgung von Osteoporose-Patienten in Deutschland - Ergebnisse der BoneEVAStudie. Dtsch Arztebl 103, 2542-2548

Häussler B, Gothe H, Gol D, Glaeske G, Pientka L, Felsenberg D (2007): The treatment and costs of osteoporosis in Germany--the BoneEVA Study. Osteoporos Int 18, 77-84

Henzen C (2013): Osteoporosw beim Mann: Bedeutung der Gonadenachse. Schweiz Med Forum 13, 768-771

Hernlund E, Svedbom A, Ivergård M, Compston J, Cooper C, Stenmark J, McCloskey EV, Jönsson B, Kanis JA (2013): Osteoporosis in the European Union: medical management, epidemiology and economic burden. Arch Osteoporos $\underline{8}, 136$

Jain S, Bilori B, Gupta A, Spanos P, Singh M (2016): Are Men at High Risk for Osteoporosis Underscreened? A Quality Improvement Project. Perm J $\underline{20}, 60-64$

Jockenhövel F, Lehnert H (2004): Östrogene für den Mann - sinnvoll oder gefährlicher Unfug? Dtsch Arztebl Int 101, 578-582

Johansson H, Azizieh F, Al Ali N, Alessa T, Harvey NC, McCloskey E, Kanis JA (2017): FRAX- vs. T-score-based intervention thresholds for osteoporosis. Osteoporos Int $\underline{28}$, 3099-3105

Johnell O, Kanis JA (2006): An estimate of the worldwide prevalence and disability associated with osteoporotic fractures. Osteoporos Int 17, 1726-1733

Kanis JA, Melton LJ, Christiansen C, Johnston CC, Khaltaev N (1994): The diagnosis of osteoporosis. J Bone Miner Res $\underline{9}, 1137-114$

Kanis JA, Johnell O, De Laet C, Johansson H, Oden A, Delmas P, Eisman J, Fujiwara S, Garnero P, Kroger H, et al. (2004a): A meta-analysis of previous fracture and subsequent fracture risk. Bone $\underline{35}, 375-382$

Kanis JA, Johansson $H$, Oden A, Johnell O, De Laet C, Eisman JA, McCloskey EV, Mellstrom D, Melton LJ, 3rd, Pols HA, et al. (2004b): A family history of fracture and fracture risk: a meta-analysis. Bone $\underline{35}, 1029-1037$

Kanis JA, Johansson H, Oden A, Johnell O, De Laet C, Melton IL, Tenenhouse A, Reeve $\mathrm{J}$, Silman AJ, Pols HA, et al. (2004c): A meta-analysis of prior corticosteroid use and fracture risk. J Bone Miner Res $\underline{19}$, 893-899

Kanis JA, Johansson H, Johnell O, Oden A, De Laet C, Eisman JA, Pols H, Tenenhouse A (2005): Alcohol intake as a risk factor for fracture Osteoporos Int $\underline{16}, 737-742$

Kanis JA, Johnell O, Oden A, Johansson H, Eisman JA, Fujiwara S, Kroger H, Honkanen R, Melton LJ, 3rd, O'Neill T, et al. (2006): The use of multiple sites for the diagnosis of osteoporosis. Osteoporos Int $\underline{17}, 527-534$ 
Kanis JA, Oden A, Johnell O, Johansson H, De Laet C, Brown J, Burckhardt P, Cooper C, Christiansen C, Cummings S, et al. (2007): The use of clinical risk factors enhances the performance of BMD in the prediction of hip and osteoporotic fractures in men and women. Osteoporos Int 18, 1033-1046

Kanis JA, Johnell O, Oden A, Johansson H, McCloskey E (2008): FRAX and the assessment of fracture probability in men and women from the UK. Osteoporos Int $\underline{19}$, 385397

Kanis JA, Oden A, Johansson H, McCloskey E (2013): Pitfalls in the external validation of FRAX: response to Bolland et al. Osteoporos Int 24, 391-392

Kanis JA, Harvey NC, Cooper C, Johansson H, Oden A, McCloskey EV (2016a): A systematic review of intervention thresholds based on FRAX : A report prepared for the National Osteoporosis Guideline Group and the International Osteoporosis Foundation. Arch Osteoporos 11, 25

Kanis JA, Compston J, Cooper C, Harvey NC, Johansson H, Oden A, McCloskey EV (2016b): SIGN Guidelines for Scotland: BMD Versus FRAX Versus QFracture. Calcif Tissue Int $\underline{98}, 417-425$

Kanis JA, Harvey NC, Johansson H, Odén A, McCloskey EV, Leslie WD (2017): Overview of fracture prediction tools Journal of clinical densitometry. J Clin Densitom 20, 444-450

Kann PH (2001): Osteodensitometrie und Ultraschalluntersuchungen des Knochens. Orthopäde $\underline{30}, 437-443$

Khosla S (2015): New Insights Into Androgen and Estrogen Receptor Regulation of the Male Skeleton. J Bone Miner Res $\underline{30}$, 1134-1137

Khosla S, Amin S, Orwoll E (2008): Osteoporosis in men. Endocr Rev 29, 441-464

Kiebzak GM, Beinart GA, Perser K, Ambrose CG, Siff SJ, Heggeness MH (2002): Undertreatment of osteoporosis in men with hip fracture. Arch Intern Med 162, 2217-2222

Lems WF (2007): Clinical relevance of vertebral fractures. Ann RheumDis $\underline{66}, 2-4$

Leitlinie Osteoporose 2006 s. DVO-Leitlinie 2006

Leitlinie Osteoporose 2009 s. DVO-Leitlinie 2009

Leitlinie Osteoporose 2014 s. DVO-Leitlinie 2014

Leitlinie Osteoporose 2017 s. DVO-Leitlinie 2017

Leslie WD, Lix LM (2011a): Absolute fracture risk assessment using lumbar spine and femoral neck bone density measurements: derivation and validation of a hybrid system. $J$ Bone Miner Res 26, 460-467

Leslie WD, Lix LM, Tsang JF, Caetano PA (2007): Single-site vs multisite bone density measurement for fracture prediction. Arch Intern Med 167, 1641-1647

Leslie WD, Lix LM, Johansson H, Oden A, McCloskey E, Kanis JA (2010): Independent clinical validation of a Canadian FRAX tool: fracture prediction and model calibration. $\mathrm{J}$ Bone Miner Res 25, 2350-2358

Leslie WD, Lix LM, Johansson H, Oden A, McCloskey E, Kanis JA (2011b): Spine-hip discordance and fracture risk assessment: a physician-friendly FRAX enhancement. Osteoporos Int 22, 839-847 
Lorentzon M, Cummings SR (2015): Osteoporosis: the evolution of a diagnosis. J Intern Med 277, 650-661

Marques A, Lucas R, Simoes E, Verstappen SMM, Jacobs JWG, da Silva JAP (2017): Do we need bone mineral density to estimate osteoporotic fracture risk? A 10-year prospective multicentre validation study. RMD Open 3, e000509

Methodenberatung der Universität Zürich,

http://www.methodenberatung.uzh.ch/de/datenanalyse.html, Zugriff am: 18.02.2018

Misiorowski W (2017): Osteoporosis in men. Prz Menopauzalny 16, 70-73

Moayyeri A, Soltani A, Tabari NK, Sadatsafavi M, Hossein-Neghad A, Larijani B (2005): Discordance in diagnosis of osteoporosis using spine and hip bone densitometry. BMC Endocr Disord $\underline{5}, 3$

Niedhart C (2016): Osteoporosis: Diagnostics and Therapy According to the German Guidelines. Z Orthop Unfall 154, 237-244

NIH Consensus Development Panel on Osteoporosis Prevention D, and Therapy. (2001): Osteoporosis prevention, diagnosis, and therapy. JAMA 285, 785-795

NIH (National Institut of Health) Osteoporosis and Related Bone Diseases - National Resource Center (2015): Osteoporosis in men, (https://www.bones.nih.gov/healthinfo/bone/osteoporosis/men (NIH Pub. No. 15-7885-E) , Zugriff am 20.02.2018)

Oden A, McCloskey EV, Kanis JA, Harvey NC, Johansson H (2015): Burden of high fracture probability worldwide: secular increases 2010-2040. Osteoporos Int 26, 2243-2248

Orwoll E, Blank JB, Barrett-Connor E, Cauley J, Cummings S, Ensrud K, Lewis C, Cawthon PM, Marcus R, Marshall LM, et al. (2005): Design and baseline characteristics of the osteoporotic fractures in men (MrOS) study--a large observational study of the determinants of fracture in older men. Contemp Clin Trials 26, 569-585

Pluijm SM, Koes B, de Laet C, Van Schoor NM, Kuchuk NO, Rivadeneira F, Mackenbach JP, Lips P, Pols HA, Steyerberg EW (2009): A simple risk score for the assessment of absolute fracture risk in general practice based on two longitudinal studies. J Bone Miner Res $\underline{24}, 768-774$

QFracture ${ }^{\circledR}$ Eingabemaske, http://www.qfracture.org/, Zugriff am 02.11.2017

Rendl S, Lapa C, Blumel C, Bundschuh RA, Schneider P (2013): Decision making for osteoporotic treatment using FRAX or DVO risk algorithms in a clinical setting. $J$ Musculoskelet Neuronal Interact 13, 339-345

Rochira V, Balestrieri A, Madeo B, Zirilli L, Granata AR, Carani C (2006): Osteoporosis and male age-related hypogonadism: role of sex steroids on bone (patho)physiology. Eur J Endocrinol 154, 175-185

Rubin KH, Friis-Holmberg T, Hermann AP, Abrahamsen B, Brixen K (2013a): Risk assessment tools to identify women with increased risk of osteoporotic fracture: complexity or simplicity? A systematic review. J Bone Miner Res 28, 1701-1717

Rubin KH, Abrahamsen B, Friis-Holmberg T, Hjelmborg JV, Bech M, Hermann AP, Barkmann R, Gluer CC, Brixen K (2013b): Comparison of different screening tools (FRAX(R), OST, ORAI, OSIRIS, SCORE and age alone) to identify women with increased risk of fracture. A population-based prospective study. Bone $\underline{56}, 16-22$ 
Sandhu SK, Nguyen ND, Center JR, Pocock NA, Eisman JA, Nguyen TV (2010): Prognosis of fracture: evaluation of predictive accuracy of the FRAX algorithm and Garvan nomogram. Osteoporos Int 21, 863-871

Shariati-Sarabi Z, Rezaie HE, Milani N, Rezaie FE, Rezaie AE (2018): Evaluation of Bone Mineral Density in Perimenopausal Period. Arch Bone Jt Surg $\underline{6}, 57-62$

Shin MH, Zmuda JM, Barrett-Connor E, Sheu Y, Patrick AL, Leung PC, Kwok A, Kweon SS, Nam HS, Cauley JA (2014): Race/ethnic differences in associations between bone mineral density and fracture history in older men. Osteoporos Int $\underline{25}, 837-845$

Siggelkow H, Hadji P (2016): Osteoporose in der Gynäkologie - Diagnostik und Therapie. Frauenheilkunde up2date $\underline{10}$, 203-218

Smith MR (2007): Androgen deprivation therapy for prostate cancer: new concepts and concerns. Curr Opin Endocrinol Diabetes Obes 14, 247-254

Tamaki J, Iki M, Kadowaki E, Sato Y, Kajita E, Kagamimori S, Kagawa Y, Yoneshima H (2011): Fracture risk prediction using $\operatorname{FRAX}(\mathrm{R})$ : a 10-year follow-up survey of the Japanese Population-Based Osteoporosis (JPOS) Cohort Study. Osteoporos Int 22, 3037-3045

Tosteson AN, Melton LJ, 3rd, Dawson-Hughes B, Baim S, Favus MJ, Khosla S, Lindsay RL, National Osteoporosis Foundation Guide (2008): Cost-effective osteoporosis treatment thresholds: the United States perspective. Osteoporos Int $\underline{19}$, 437-447

Tu KN, Lie JD, Wan CKV, Cameron M, Austel AG, Nguyen JK, Van K, Hyun D (2018): Osteoporosis: A Review of Treatment Options. P T $\underline{43}, 92-104$

Vestergaard P (2007): Discrepancies in bone mineral density and fracture risk in patients with type 1 and type 2 diabetes--a meta-analysis. Osteoporos Int $\underline{18}, 427-444$

Weaver CM, Gordon CM, Janz KF, Kalkwarf HJ, Lappe JM, Lewis R, O'Karma M, Wallace TC, Zemel BS (2016): The National Osteoporosis Foundation's position statement on peak bone mass development and lifestyle factors: a systematic review and implementation recommendations. Osteoporos Int $\underline{4}, 1281-1386$

WHO (1991): Consensus development conference: prophylaxis and treatment of osteoporosis. Am J Med 90, 107-10

WHO (2003): Prevention and management of osteoporosis. World Health Organ Tech Rep Ser 921, 1-164,

Yan Z, Huang H, Li J, Wang J (2016): Relationship between subclinical thyroid dysfunction and the risk of fracture: a meta-analysis of prospective cohort studies. Osteoporos Int 27, $115-125$

Zhang X, Yu Z, Yu M, Qu X (2015): Alcohol consumption and hip fracture risk. Osteoporos Int $\underline{26}, 531-542$ 


\section{Danksagung}

Frau Prof. Dr. Siggelkow danke ich für die herzliche Integration in ihre Abteilung, ihr Vertrauen und das Überlassen dieser Arbeit. Ich danke ihr für die hervorragende Betreuung und die motivierenden Diskussionen. Sie unterstütze das Projekt stets mit den richtigen Ratschlägen und förderte mich auch über diese Arbeit hinaus.

Frau Anna Giessel danke ich für die freundschaftliche Zusammenarbeit an diesem Projekt.

Herrn Prof. Dr. C. Glüer danke ich für die Bereitstellung des nicht publizierten Scores und seinem stets produktiven Feedback.

Dem gesamten Team des MVZ endokrinologikum Göttingen danke ich für die Unterstützung der Arbeit, die Hilfsbereitschaft und die professionelle Aufnahme.

Zuletzt möchte ich meiner Familie für ihre immerwährende Unterstützung, die mein Studium und diese Promotion überhaupt erst ermöglicht haben, von Herzen danken. 